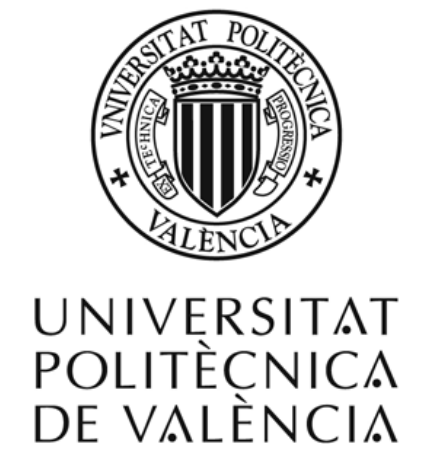

\title{
Metodología para la parametrización en tiempo real del proceso de combustión en MCIA.
}

\author{
TESIS DOCTORAL \\ Realizada por: \\ Ali Abbad Andaloussi \\ Dirigida por: \\ Dr.D. Jose Manuel Lujan Martinez
}

DEPARTAMENTO DE MÁQUINAS Y MOTORES TÉRMICOS de la

UNIVERSIDAD POLITÉCNICA DE VALENCIA

Valencia, 1 de Julio del 2013 



\section{TESIS DOCTORAL}

Metodología PARA LA PARAMETRIZACIÓN EN TIEMPO REAL DEL PROCESO DE COMBUSTIÓN EN MCIA.

Realizada por: D. Ali Abbad Andaloussi

Dirigida por: Dr. D. Jose Manuel Lujan Martinez

\section{TRIBUNAL CALIFICADOR:}

Presidente: $\quad$ Dr. D. Francisco Payri González

Secretario: Dr. D. Carlos Guardiola García

Vocales: $\quad$ Dr. D. Octavio Armas Vergel

Dr. D. Andres Melgar Bachiller

Dr. D. Pedro Acisclo rodriguez Aumente

Vocales suplentes:

Dra. D. Juan-Jose Hernandez Adrover

Dr. D. Francisco Vicente Tinaut Fluixa

Valencia, 01 de Julio de 2013 



\section{Resumen}

El trabajo desarrollado en la presente tesis doctoral está encaminado al desarrollo de metodologías para la parametrización en tiempo real del proceso de combustión en motores Diésel de inyección directa. Para ello, se dispone del conocimiento previo sobre adquisición de señal de presión y diagnóstico de la combustión mediante el modelado termodinámico existente en el grupo de trabajo en que se ha desarrollado.

El trabajo se ha orientado, por un lado, a definir y poner a punto la herramienta necesaria para la medida y el tratamiento digital de la presión en cámara con el objetivo de aportar mayor fiabilidad y precisión al modelo termodinámico de diagnóstico de la combustión y por otro lado, al desarrollo de nuevas metodologías de diagnóstico de la combustión basadas en el análisis de la derivada de la presión en cámara.

De este modo, el trabajo realizado ha llevado a la obtención de procedimientos simples que permiten localizar el ángulo de inicio de la combustión, el ángulo de final de la combustión y el ángulo donde se produce la máxima tasa de liberación de calor únicamente a partir de la señal del sensor de presión en cámara.

Además, se ha evaluado el potencial y se ha propuesto ámbitos de aplicación de cada metodología.

El trabajo realizado aporta precisión y robustez a la caracterización de la combustión, abriendo la posibilidad de desarrollos futuros de estrategias de control basadas en la optimización, en tiempo real, del proceso de la combustión. Una optimización crucial para la mejora de los motores diésel y la reducción de los contaminantes. 



\section{Resum}

El treball elaborat a la present tesis doctoral està encaminat al desenvolupament de metodologies per a la parametrització en temps real del procés de combustió en motors Diesel d'injecció directa. Per a portar-ho a terme, es disposa del coneixement previ sobre adquisició de senyal de pressió i diagnòstic de la combustió mitjançant el modelat termodinàmic existent al grup de treball que ha format part de la investigació.

El treball s'ha orientat, per una banda, a definir i posar a punt les eines necessàries per a la mesura $\mathrm{i}$ el tractament digital de la pressió en càmera amb l'objectiu d'aportar la major fiabilitat i precisió al model termodinàmic de diagnòstic de la combustió i per altra banda, al desenvolupament de noves metodologies de diagnòstic de la combustió obtinguda en el anàlisis de la derivada de la pressió en càmera.

D'aquesta manera, el treball realitzat ha donat lloc a l'obtenció de procediments simples que permeten localitzar l'angle d'inici de la combustió, l'angle de final de la combustió i l'angle on es produeix la màxima taxa d'alliberament de calor únicament a partir de la senyal del sensor de pressió en càmera

A més, s'ha avaluat el potencial i s'han proposat àmbits d'aplicació en cadascuna de les metodologies.

El treball realitzat aporta precisió i robustesa a la caracterització de la combustió, obrint la possibilitat de desenvolupar futures estratègies de control basades en l'optimització en temps real del procés de la combustió. Una optimització crucial per a la millora dels motors Diesel i la reducció dels contaminants. 



\section{Abstract}

The research presented in this thesis is focused on the development of methodologies for the real time parametrisation of the combustion process in direct injection diesel engines. The start point is the know how concerning pressure signal acquisition and combustion diagnosis using thermodynamic models, previously developed by the research group.

The research carried out is focused on two main topics. First, the design and development of the required tool for the acquisition and digital processing of the in-cylinder pressure, with the objective of improving the reliability and precision of the existing thermodynamic model used for combustion diagnosis. As a second step, it includes the development of new methodologies for combustion diagnosis based on the analysis of the in-cylinder pressure derivative.

Thus, the research includes the implementation of simple methods to identify the start and end of combustion and the maximum rate of heat release angles using only the in-cylinder pressure sensor signal. These methods have been validated in a wide range of applications, including real-time, on-line and off-line combustion diagnosis, and their applicability has been confirmed.

Finally, the research also increases the accuracy and reliability of the current combustion characterization methods, widening the possibilities of future development of control strategies based on real-time optimisation of the combustion process, which is expected to be a key aspect for the improvement of Diesel engines and pollution reduction. 

A Aws

$\forall$ HN2 



\section{Agradecimientos}

Definitivamente este trabajo no se habría podido realizar sin la colaboración de muchas personas que me brindaron su ayuda; siempre resultará difícil agradecer a todos aquellos que de una u otra manera me han acompañado para el desarrollo de esta investigación, porque nunca alcanza el tiempo, el papel o la memoria para mencionar o dar con justicia todos los créditos y méritos a quienes se lo merecen. Por tanto, quiero agradecerles a todos ellos cuanto han hecho por mí, para que este trabajo saliera adelante de la mejor manera posible.

Gracias a quien me lo ha dado todo.

Gracias a mi director, Jose Manuel Lujan, por ser pilar fundamental para afianzar este sueño, por su confianza, apoyo y sus críticas, así como todo lo que de él he aprendido, personal y profesionalmente.

Gracias al impresionante grupo de trabajo CMT que me brindó su apoyo y toda su colaboración para la realización de este trabajo, en particular a Francisco Payri, jefe y ejemplo a seguir, quien permanentemente me guió con su espíritu alentador.

Gracias Jaime por las consultas, los comentarios y los aportes. Mil gracias.

Gracias a las fuentes de conocimiento: Jose María Desantes, Jean Arrègle, Carlos Guardiola, etc. por su sus consejos y dedicación.

Gracias a todos los amigos del departamento, los que siguen aquí y a los que ya no están, que durante todos estos años la convivencia con ellos ha sido lo mejor de la tesis doctoral.

Gracias a mis amigos por lo buenos momentos, el toque especial y único que dan a mi vida, en especial a Victor por la revisión y la corrección del texto final de la tesis.

Por ultimo, gracias Sanae por iluminar mi camino, por tu paciencia y por no dudar nunca de mi. Sé que tengo muchos defectos pero acabando la tesis tendré uno menos. 

La presión es imprevisible. Puede convertir el carbón en polvo o en diamante. 



\section{Índice general}

1. Planteamiento de la tesis 1

1.1. Introducción y justificación . . . . . . . . . . . . . . . 3

1.2. Antecedentes . . . . . . . . . . . . . . . 6

1.2.1. Principales estrategias de control en motores diésel . . . . . . . 7

1.2.2. Diagnóstico de la combustión . . . . . . . . . . . . . . . . . . 10

1.3. Objetivos y Metodología . . . . . . . . . . . . . . . . . . . 11

1.4. Estructura de la tesis . . . . . . . . . . . . . . . . . . . . . . . . . . . . . . .

Bibliografía del capítulo . . . . . . . . . . . . . . . . 13

2. Técnicas utilizadas para medida y control de MCIA 17

2.1. Introducción . . . . . . . . . . . . . . . . . . . . . . . . . . 19

2.2. Plataforma . . . . . . . . . . . . . . . 21

2.2.1. El microcontrolador . . . . . . . . . . . . . . . . 21

2.2.2. Acondicionamiento de señales . . . . . . . . . . . . . . . . 22

2.2.3. Limitaciones . . . . . . . . . . . . . . . . 22

2.3. Variables a medir y sensores . . . . . . . . . . . . . 23

2.3.1. Sensores de medida de temperaturas . . . . . . . . . . . 25

2.3.2. Sensores de medida de presión . . . . . . . . . . . . . . . 26

2.3.3. Sensor de medida de régimen de giro . . . . . . . . . . . . . . . 26

2.3.4. Sensor de caudal de masa de aire . . . . . . . . . . . . . . . 27

2.3.5. Sensor de concentración de oxígeno . . . . . . . . . . . . 27

2.3.6. Sensores de posición o desplazamiento . . . . . . . . . . . . 27

2.3.7. Otros sensores y tendencias . . . . . . . . . . . . . 28

2.4. Actuadores . . . . . . . . . . . . . . . . . . . 29

2.5. Estrategias de control . . . . . . . . . . . . . . . . . . . . . . . . . . . . . . . . 30

2.6. Las tendencias futuras . . . . . . . . . . . . . . . . . . . . . . . . . . . . . . . 33

Bibliografía del capítulo . . . . . . . . . . . . . . . . . . 34 
3. Utilizaciones específicas de la presión en cilindro 39

3.1. Introducción . . . . . . . . . . . . . . . . . . . 41

3.2. Uso de la presión en cámara . . . . . . . . . . . . . . . . . . . . . . . . . . . . . 43

3.2.1. Presión máxima y ángulo de presión máxima. . . . . . . . . . . . 45

3.2.2. Presión media indicada . . . . . . . . . . . . . . . . . . 46

3.2.3. Diagrama $\log (p) / \log (V) \ldots \ldots \ldots$. . . . . . . . 46

3.2.4. Gradiente de presiones . . . . . . . . . . . . . . . . . 47

3.2.5. Parámetros de la Combustión . . . . . . . . . . . . . . . . . . . . . . . . . 47

3.3. Sistemas de medida de la Presión en cámara . . . . . . . . . . . . . . . . 49

3.3.1. Sensores y Acondicionadores . . . . . . . . . . . . . . . 49

3.3.1.1. Parámetros para elección de sensores de presión . . . . 49

3.3.1.2. Sensores de presión en cámara Piezorresistivos . . . . . 51

3.3.1.3. Sensores de presión en cámara ópticos . . . . . . . . . 52

3.3.1.4. Sensores de presión en cámara piezoeléctricos . . . . . 54

3.3.2. Características de la señal . . . . . . . . . . . . . . 56

3.3.3. Criterios de selección del sistema de medida . . . . . . . . . 62

3.3.3.1. Resolución. . . . . . . . . . . . . . . 63

3.3.3.2. Frecuencia de muestreo. . . . . . . . . . . . 65

Bibliografía del capítulo . . . . . . . . . . . . . . . . . 68

4. Fuentes de información experimental y teórica.

4.1. Motores y salas de ensayo. Descripción general. . . . . . . . . . . . . . . 77

4.2. Sistema de medida de las variables instantáneas. . . . . . . . . . . . . . . 80

4.3. Determinación de la ley de liberación de calor. . . . . . . . . . . . . . . . . . 82

4.3.1. Parámetros del motor . . . . . . . . . . . . . 83

4.3.2. Ecuación de los gases perfectos . . . . . . . . . . . . 85

4.3.3. Balance energético . . . . . . . . . . . . . . . . . . . . . . . . 86

4.3.4. Balance másico . . . . . . . . . . . . . . . . . . 87

4.3.5. Parámetros de interés para el desarrollo de la tesis. . . . . . . . . 88

Bibliografía del capítulo . . . . . . . . . . . . . . . . 93

5. Aporte al tratamiento de la presión en cámara para el diagnóstico de la combustión.

5.1. Introducción . . . . . . . . . . . . . . . . . . . . . 97

5.2. Etapa 1. Promediado . . . . . . . . . . . . . . . . . 98

5.2.1. Propuesta para definir el número de ciclos a promediar . . . . . . 105

5.3. Etapa 2. El filtrado digital de la señal de presión . . . . . . . . . . . . . . 111

5.3.1. Métodos experimentales para determinar los parámetros del filtro 114

5.3.2. Propuesta para determinar los parámetros del filtro . . . . . . . 115

5.3.3. Propuesta para el filtrado de la señal de presión en cilindro en tiempo real. . . . . . . . . . . . . . . . . . 118

5.4. Propuesta para la medida de la derivada de la presión en cámara . . . . . 119 Bibliografía del capítulo . . . . . . . . . . . . . . . . . . 123 
6. Propuestas de métodos de parametrización de la combustión en tiempo real $\mathbf{1 2 7}$

6.1. Introducción . . . . . . . . . . . . . . . . . . . . . . . . . 129

6.2. Síntesis y planteamiento de las metodologías . . . . . . . . . . . . 130

6.3. Metodología de diagnóstico de la combustión a partir del análisis temporal de la $d p \ldots \ldots$. . . . . . . . . . . . . . . . . . 133

6.3.1. Herramientas de análisis de la señal en dominio temporal . . . . . 133

6.3.2. Aplicación del método basado en el análisis temporal de la derivada de la presión . . . . . . . . . . . . . 136

6.3.3. Validación, análisis comparativo detección del SoC . . . . . . . . 140

6.3.3.1. Caso de puntos de funcionamiento del motor con una inyección. . . . . . . . . . . . . 140

6.3.3.2. Caso de puntos de funcionamiento del motor con varias inyecciones. . . . . . . . . . . . . 141

6.3.4. Validación, análisis comparativo detección del EoC . . . . . . . . 150

6.3.5. Validación, análisis comparativo detección del ángulo de máxima dFQL . . . . . . . . . . . . . . . . 153

6.3.6. Implementación en tiempo real. . . . . . . . . . . . . . . . 157

6.4. Metodología de diagnóstico de la combustión a partir del análisis en dominio de frecuencias de la $d p . \ldots . \ldots 161$

6.4.1. Herramientas de análisis conjuntas de la señal en tiempo y frecuencia . . . . . . . . . . . . . . . 161

6.4.2. Aplicación de los métodos basados en el análisis en frecuencias de la $d p \ldots \ldots \ldots$. . . . . . . . . . . . . . . . . 163

6.4.3. Aplicación de los métodos basados en el análisis conjunto Tiempo-Frecuencias de la $d p . \quad$. . . . . . . . . . . . . . . . . . . 169

6.5. Análisis comparativo de las metodologías propuestas y conclusiones. . . . 173 Bibliografía del capítulo . . . . . . . . . . . . . . . . 176

7. Conclusiones y trabajos futuros 179

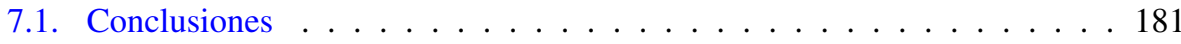

7.2. Trabajos futuros . . . . . . . . . . . . . . . . . 184

$\begin{array}{ll}\text { Bibliografía general } & 185\end{array}$ 



\section{Tabla de símbolos}

\section{Griegos}

$\alpha \quad$ Ángulo de giro del cigüeñal

$\Delta \alpha_{m} \quad$ Desfase por pérdidas de masa

$\Delta \alpha_{Q} \quad$ Desfase por pérdidas de calor

$\Delta \alpha_{t} \quad$ Desfase termodinámico

$\Delta h_{\text {vap }} \quad$ Entalpía de vaporización del combustible

$\Delta \quad$ Resolución de la muestra

$\gamma \quad$ Cociente de calores específicos (exponente adiabático)

$\sigma \quad$ Desviación típica

$\tau_{\text {vap }} \quad$ Tiempo de evaporación

$\tau_{\text {retraso }}$ Tiempo de retraso

\section{Latinos}

$\dot{m} \quad$ Gasto másico

$c_{p} \quad$ Calor específico a presión constante

$c_{v} \quad$ Calor específico a volumen constante

$C_{W 1}, C_{W 2}$ Constantes de la ecuación de transmisión de calor de Woschni

D Diámetro del cilindro 
$d p \quad$ Derivada de presión en cámara

$[\mathrm{bar} /]$

$f \quad$ Frecuencia o frecuencia normalizada respecto a la frecuencia de muestreo $[\mathrm{Hz},-]$

$f_{m} \quad$ Frecuencia de muestreo

$[\mathrm{Hz}]$

$f_{n} \quad$ Frecuencia natural

$[\mathrm{Hz}]$

$i \quad$ Indice de la muestra

$k \quad$ Indice del armónico

$K_{\text {def }} \quad$ Coeficiente de deformaciones

$L_{b} \quad$ Longitud de la biela

$L_{m} \quad$ Longitud de la manivela

M Número de muestras total adquiridas

$[-]$

$m \quad$ Masa

$[k g]$

$n \quad$ Exponente politrópico

$n_{c} \quad$ Número de ciclos de presión consecutivos adquiridos

$n_{p c} \quad$ Número de muestras adquiridas en cada ciclo de presión

$P \quad$ Potencia

p Presión

PM Peso molecular

$Q \quad$ Calor transmitido a las paredes de motor

$q \quad$ Carga eléctrica

$R \quad$ Constante específica de los gases

$r \quad$ Señal de ruido

rc Relación de compresión

rpm Régimen de giro del motor

$s \quad$ Señal medida

$T \quad$ Temperatura

$t \quad$ Tiempo 
U Tensión o voltaje eléctrico

$u \quad$ Energía interna específica

V Volumen

$V_{d} \quad$ Volumen desplazado

$Y \quad$ Fracción másica

z Número de cilindros

\section{Subíndices y superíndices}

a Relativo al aire

$a, q \quad$ Relativo al aire quemado estequiométricamente

adm Relativo a la admisión

amb Relativo a las condiciones ambientales

amp Relativo al amplificador de carga

bb Relativo al blow-by

bowl Relativo al bowl

c Relativo al armónico o frecuencia de corte

CA Relativo al ciclo abierto

carter Relativo al cárter

cil Relativo al cilindro

comb Relativo a la combustión

cul Relativo a la culata

def Relativo a las deformaciones

evap Referido a la evaporación

f Relativo al combustible

$f, e v \quad$ Relativo al combustible evaporado

$f, g \quad$ Relativo al combustible gaseoso

$f$, iny Relativo al combustible líquido en condiciones de inyección 
$f, l \quad$ Relativo al combustible líquido

$f, q \quad$ Relativo al combustible quemado estequiométricamente

$g \quad$ Relativo al gas

iny Relativo a la inyección de combustible

$\max \quad$ Valor máximo

min Valor mínimo

n Relativo al exponente politrópico

oil Relativo al aceite lubricante

p Relativo a las paredes de la cámara (pistón, culata y cilindro)

pil Relativo a la inyección piloto

prin Relativo a la inyección principal

ref Valor de referencia

res Relativo a los gases residuales

vap Relativo a la vaporización

\section{Siglas}

$C A / D$ Relativo a la conversión Analógico-Digital de señales

CALMEC Código comercial para el diagnóstico de la combustión a partir de la presión en cámara

$d F Q L \quad$ Tasa de calor liberado

$[\mathrm{J} /,-/]$

DI Inyección directa (del inglés Direct Injection)

$F Q L \quad$ Calor liberado acumulado

IFFT Transformada rápida de Fourier inversa (del inglés Inverse Fast Fourier Transform)

IIR Filtros digitales recursivos de respuesta infinita al impulso (del inglés Infinite Impulse Response)

pmi Presión Media Indicada

SNR Relación señal-ruido (del inglés Signal to Noise Ratio) 
$S N R_{Q} \quad$ Relación señal-ruido de cuantización

DFP Filtros de partículas (del inglés Diésel Particulate Filter)

DOC Catalizadores(del inglés Diésel Oxidation Catalyst)

ECU Unidad de control electrónica (del inglés Electronic Control Unit)

EGR Recirculación de gases de escape (del inglés Exhaust Gas Recirculation)

EoI Final de la inyección (del inglés End Of Injection)

FFT Transformada rápida de Fourier (del inglés Fast Fourier Transform) [-]

FIR Filtros digitales no recursivos de respuesta finita al impulso (del inglés Finite Impulse Response)

HCCI Motor de encendido por compresión con carga homogénea (del inglés Homogeneous Charge Compresion Ignition)

JTFA El análisis conjunto de tiempo-frecuencia (del inglés Joint Time-Frequency Analysis)

LTC Motor de combustiones frías (del inglés Low Temperature Combustion)

MCIA Motor de Combustión Interna Alternativo $\quad[-]$

MEC Motor de Encendido por Compresión [-]

MEP Motor de Encendido Provocado [-]

MIF Frecuencia media instantánea (del inglés Mean Instantaneous Frequency)

PCCI Motor de encendido por compresión con carga premezclada (del inglés Premixed Charge Compression Ignition)

PID Algoritmo de control función de la Proporcional, la Integral y la derivada del error entre la consigna y la medida.

PMI Punto Muerto Inferior. Localizado en $\alpha= \pm 180$

PMS Punto Muerto Superior. Localizado en $\alpha=0$

RCA Ángulo de Retraso del Cierre de la(s) válvula(s) de Admisión []

SCR Catalizadores selectivos(del inglés Selective Catalyst Reduction)

SoC Inicio de la combustión (del inglés Start Of Combustion)

SoI Inicio de la inyección (del inglés Start Of Injection) 
STFT Transformadas de Fourier de corta duración (del inglés Short Time Fourier Transform)

TDF Transformada Discreta de Fourier

$\mathrm{TDFb}$ Transformada Discreta de Fourier bidimensional

TGV Turbina de Geometría Variable $\quad$ [- ] 
Capítulo 1

\section{Planteamiento de la tesis}




\section{Índice}

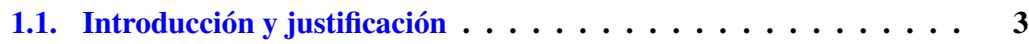

1.2. Antecedentes .................... 6

1.2.1. Principales estrategias de control en motores diésel . . . . . 7

1.2.2. Diagnóstico de la combustión . . . . . . . . . . . . 10

1.3. Objetivos y Metodología . . . . . . . . . . . . . . 11

1.4. Estructura de la tesis $\ldots \ldots \ldots \ldots \ldots$

Bibliografía del capítulo . . . . . . . . . . . . . . . . 13 


\subsection{Introducción y justificación}

La primera crisis del petróleo en 1973 manifestó la importancia del ahorro del combustible. A raíz de ello, muchos gobiernos establecieron acciones para limitar el consumo de combustible, a la vez que los fabricantes de motores empezaron a interesarse por la producción de motores menos contaminantes.

En junio de 1974 la revista Nature publicó un artículo de Paul Crutzen y María Molina (Premio Nobel de Química 1995), en el que se alertó a la comunidad internacional de la forma en que estaba debilitando la capa de ozono.

En 1988 se creó un nuevo organismo, el "Grupo Intergubernamental de Expertos sobre el Cambio Climático" o "Panel Intergubernamental del Cambio Climático" (IPCC). Su labor consiste en evaluar los datos científicos sobre el cambio climático, evaluar los impactos sociales, económicos y ecológicos, y la elaboración de acciones de prevención y estrategias de respuesta.

En 1990, la Conferencia Mundial sobre el clima en Ginebra ya había reconocido el cambio climático como un problema en todo el mundo a una escala sin precedentes.

En junio de 1992, en Río de Janeiro, la ONU celebró su segunda conferencia sobre el medio ambiente y desarrollo, la cual representó un hito para el gran alcance de los principios que se adopten en una declaración final en la que se destaca que el desarrollo y la protección del medio ambiente son inseparables. La declaración de Río instó a las naciones a cooperar como socios a nivel mundial con el fin de conservar, proteger y restablecer la salud y la integridad de los ecosistemas de la Tierra.

En 1995, el segundo informe del IPCC confirmó los temores expresados en Río. El calendario de las negociaciones internacionales elaborados en Río siguió adelante y en diciembre de 1997, la conferencia sobre el cambio climático de Kyoto se llevó a cabo con el fin de llegar a un acuerdo sobre objetivos de reducción de gases de efecto invernadero.

Todos estos acontecimientos han sido los principales propulsores de la evolución de los motores hacia maquinas más respetuosas con el medio ambiente. Las diferentes pautas que han sido indicadas para la reducción del consumo de combustible y las emisiones a la atmósfera, como las presentadas en la tabla (1.1), no podrían conseguirse sin el aporte de nuevas tecnologías y principalmente la gestión electrónica de los motores.

\begin{tabular}{|l|c|c|c|c|c|c|c|c|c|}
\cline { 2 - 10 } \multicolumn{2}{c|}{} & \multicolumn{2}{c|}{ CO } & \multicolumn{2}{c|}{ HC+NOx } & \multicolumn{2}{c|}{ NOx } & \multicolumn{2}{c|}{ Partículas } \\
\cline { 2 - 10 } & Año & $\mathrm{g} / \mathrm{km}$ & $\%^{(*)}$ & $\mathrm{g} / \mathrm{km}$ & $\%^{(*)}$ & $\mathrm{g} / \mathrm{km}$ & $\%^{(*)}$ & $\mathrm{g} / \mathrm{km}$ & $\%^{(*)}$ \\
\hline \hline Euro I & 1992 & 2.72 & $* *$ & 0.97 & $* *$ & $* *$ & $* *$ & 0.14 & $* *$ \\
\hline Euro II & 1996 & 1 & 63 & 0.7 & 28 & $* *$ & $* *$ & 0.08 & 43 \\
\hline Euro III & 2000 & 0.64 & 36 & 0.56 & 20 & 0.5 & $* *$ & 0.05 & 38 \\
\hline Euro IV & 2005 & 0.5 & 22 & 0.3 & 46 & 0.25 & 50 & 0.025 & 50 \\
\hline Euro V & 2009 & 0.5 & 0 & 0.23 & 23 & 0.18 & 28 & 0.005 & 80 \\
\hline Euro VI & 2014 & 0.5 & 0 & 0.17 & 26 & 0.08 & 56 & 0.005 & 0 \\
\hline
\end{tabular}

(*) Reducción respecto a la normativa anterior

Tabla 1.1: Evolución de la normativa europea sobre emisiones (motores diésel turismos) 
Desde la implementación de las Unidades Electrónicas de Control (ECUs) en los motores de automoción, se ha abierto un amplio abanico de estrategias de control para optimizar el funcionamiento de estos motores. Hoy en día, el control electrónico, mediante dichas unidades, ha permitido a los motores diésel convertirse en la alternativa más eficiente para aplicaciones de automoción.

Además de diagnosticar fallos, las ECUs cumplen el papel principal de llevar el motor a su punto óptimo de funcionamiento, evaluado mediante una serie de medidas de sensores y cálculos internos, manejando una serie de actuadores. De esta forma, entre otros, encontramos que los aportes principales de las ECUs son:

- Evaluar el estado del motor vigilando en cada instante las diferentes magnitudes físicas medibles. Lecturas de sensores como el de régimen de giro, de posición del acelerador, de masa de aire, de presión de admisión, de presión de combustible y de temperatura de motor, son interpretadas por la ECU diagnosticando así posibles fallos o anomalías en el funcionamiento del motor.

- Optimizar el punto de funcionamiento del motor, imponiendo los reglajes establecidos por el fabricante y decidiendo las consignas adecuadas de varias variables que aportan mejor rendimiento al motor sin excluir la disminución de los contaminantes. Actuadores como la turbina de geometría variable (TGV), válvula de recirculación de gases de escape (EGR), common rail e inyectores, responden a las solicitudes de la ECU para llevar el motor a los puntos de funcionamiento prediseñados para mejorar la eficiencia del motor a la vez que respetar las diferentes normativas cada vez más exigentes en cuanto a emisiones.

Hoy en día, el reto principal de los fabricantes de motores es lograr el mejor rendimiento de los motores cumpliendo con las normas de las emisiones de contaminantes cada vez más exigentes (tabla (1.1)). Para ello, existen dos vías principales y, a su vez, complementarias:

- La incorporación de nuevos sistemas, como la implementación de dispositivos de post-tratamiento como trampas de partículas, catalizadores, inyectores de aire en el escape, etc.

- La optimización de las estrategias de control implementando nuevos sensores, nuevos algoritmos de control y mejores actuadores. Siendo un reto crítico el control del proceso de combustión. 
De hecho, dado que la forma en que se produce la combustión afecta directamente a las emisiones y las prestaciones del motor, el análisis del proceso de la combustión sigue siendo una de las herramientas más utilizadas en la investigación para el desarrollo y la evaluación de los motores. Sin embargo, en las estrategias de gestión de los motores, la evaluación del proceso de combustión se encuentra casi ausente, debido fundamentalmente a tres factores:

1. Escasez de herramientas experimentales para la puesta a punto de nuevas estrategias de control.

2. El coste de los sensores adicionales necesarios para aportar las variables necesarias para una buena evaluación de la combustión.

3. El alto coste computacional que supone un diagnóstico instantáneo del proceso de la combustión.

El primer factor es debido a la falta de una herramienta adecuada para comprobar varias estrategias propuestas en los foros científicos, donde hay un acuerdo en que la incorporación de parámetros de la combustión permite hacer controles avanzados que mejoran tanto el comportamiento de los motores diésel como sus emisiones. De hecho, los fabricantes de sistemas de adquisición de datos para laboratorios de investigación de motores ofrecen cada vez más herramientas para diagnóstico de combustión online. Sin embargo, bien por su alto precio o bien por la limitadas opciones que permiten sus algoritmos de diagnóstico de combustión, su uso sigue estando al alcance de muy pocos y sobretodo no es viable su uso en los motores de serie.

El segundo factor, es cada vez menos crítico debido a la aparición de sensores de bajo coste.

El tercer factor precisa el uso de submodelos fiables y de algoritmos sencillos para disminuir la carga computacional de la ECU.

Con estas premisas, se presenta en esta tesis varios métodos sencillos para el diagnóstico de la combustión online a partir de la señal del sensor de presión en cámara. Métodos que pueden ser implementados tanto en tiempo real, en las ECUs de serie de los motores comerciales, como en los laboratorios de investigación. Es decir, herramientas útiles para control y desarrollo de motores diésel. 


\subsection{Antecedentes}

Hasta 1970, el control de la renovación de carga y la alimentación del combustible se producía fundamentalmente de forma mecánica. La introducción del control electrónico de la inyección del combustible en los motores de encendido provocado supuso el inicio de la gestión electrónica de los motores.

Esta introducción fue una consecuencia directa de la necesidad de reducir consumo de combustibles y las emisiones. De hecho, la primera estrategia de control electrónico fue implementada en 1976, en la unidad de control "Monotronic" de la factoría Bosh. Combinaba el control de la inyección de la gasolina, el control con sonda lambda y catalizador de tres vías.

Hasta estas fechas, una gran parte de los sistemas de inyección en motores diésel utilizaban cámaras de premezcla donde se producía la inyección del combustible, antes de introducir la mezcla en el cilindro. Aunque ya se disponía de sistemas de inyección diésel desde los años 30, el control en los años 70 seguía siendo totalmente mecánico.

Mientras que el uso de la gestión electrónica de los motores gasolina se hacía cada vez más importante, la introducción de la primera unidad de control electrónico para la gestión los sistemas de inyección en motores diésel, no se consiguió hasta pasado el año 1982. El desarrollo de los turbogrupos en los años 80 y las grandes ventajas que proporcionaba la gestión electrónica de la inyección forzó la implementación definitiva en 1990 de las primeras unidades de control electrónico en motores diésel.

A finales de los años 90, la aparición del common-rail, los inyectores piezoeléctricos, la disminución del coste de los sensores, la mejora notable en la capacidad de los microcontroladores y la imposición de la implementación de sistemas de diagnóstico a bordo (OBD), forzaron la generalización del uso de las ECUs.

La implementación de cualquier función para el control de motores actuales, requiere un conocimiento de las prestaciones de las ECUs, de los sensores, actuadores y la filosofía de los algoritmos de control ya implementados.

La fiabilidad de la mayoría de las estrategias de control y el carácter óptimo de los puntos de funcionamiento de los motores son a menudo validados en centros de investigación, mediante la evaluación y el análisis de los fenómenos que se producen dentro de la cámara de combustión. Para ello, el diagnóstico de la combustión basado en la presión en cámara sigue siendo la herramienta principal para el análisis y el entendimiento de dichos fenómenos. Esto estimula el desarrollo de nuevas metodologías de diagnóstico de la combustión, como las que se presentan en la presente tesis, para facilitar su aplicación en el control en tiempo real de los motores diésel. 


\subsubsection{Principales estrategias de control en motores diésel}

En las últimas décadas, el uso del control electrónico en los motores se ha extendido notablemente. El avance vertiginoso de los microcontroladores ha permitido gestionar la totalidad de los sistemas que componen los motores actuales. De hecho, la ECU no deja de ser un microcontrolador de altas prestaciones con sus periféricos de entradas y salidas adaptadas para medir las diferentes variables físicas y actuar sobre los sistemas vigilados.

Los sistemas a controlar pueden dividirse en sistema de diagnóstico del estado del motor, sistema de gestión del aire o renovación de la carga y sistema de control de la inyección. Estos sistemas disponen de una serie de sensores mediante los que se obtienen medidas de variables físicas aptas para ser utilizadas en una determinada estrategia programada de actuación.

En la figura(1.1) se puede observar un esquema general básico de los diferentes sistemas de control utilizados en motores diésel, distinguiendo los siguientes:

- Sistema de gestión del aire que tiene como objetivo controlar la masa de aire limpio y la masa de gases recirculados (EGR) introducidos en el cilindro. Para ello, con el objetivo de regular la presión de sobrealimentación $\left(P_{2}\right.$ o $\left.P_{a d m}\right)$ se actúa sobre el sistema de turbo-alimentación mediante la turbina de geometría variable (TGV) o wastegate y para regular el caudal de los gases recirculados se actúa sobre la apertura de la válvula de EGR estimando dicho caudal con la ayuda de la medida de aire admitido.

- El sistema de control de la inyección que a partir del estado del motor y la demanda del conductor (posición del pedal del acelerador) decide la cantidad de combustible necesaria $\left(m_{f u e l}\right)$. Esta cantidad es traducida a una presión de inyección lograda con el control del sistema de inyección (comúnmente Common-rail o bomba inyectora), un número de inyecciones, un instante y una duración de cada inyección.

Dependiendo de las características del sistema a controlar, podemos encontrar tres tipos comunes de estrategias:

Control dos posiciones, o control todo o nada trata de la activación / desactivación de un sistema comparando la variable medida con un valor de referencia. Por ejemplo, activar el sistema de refrigeración al superar la temperatura del motor $90^{\circ} \mathrm{C}$.

Control en bucle abierto, o imposición de un valor de actuación en función de una combinación de varias variables medidas que alimentan unas tablas programadas $\mathrm{y}$, mediante interpolaciones, se decide el valor de la acción de control. Estos algoritmos son con diferencia los más utilizados para la gestión de los motores diésel. Por ejemplo, duración de la inyección que se interpola entre valores de tablas multidimensionales siendo las dimensiones variables como la presión de inyección, régimen de giro, cantidad de combustible demandada, temperatura de combustible, etc. Estas estrategias no son realimentadas, es decir, no disponen de información sobre si el sistema ha llegado al estado requerido o no. 
Control en bucle cerrado, generalmente mediante algoritmos de control re-alimentados basados en aplicar una acción de control resultante de la suma de una acción proporcional al error de la medida respeto a la consigna deseada, su integral y su derivada (control PID). Estos algoritmos son los empleados, por ejemplo, en el control de la renovación de carga. Para mejorar el comportamiento de los sistemas controlados en bucles cerrado, varios estudios están dirigidos hacía remplazar los algoritmos de control PID por algoritmos más avanzados como los controles predictivos o redes neuronales.

Si antes la mayoría de las estrategias de control eran aplicadas mediante actuadores mecánicos (termostatos, válvulas mecánicas, etc.), hoy en día, y debido a la variedad de los sensores de la que disponen los motores diésel y las altas prestaciones de las unidades de control electrónico, se tiende a implementar la mayoría de las estrategias de control mediante software. Eso abre la posibilidad de implementar estrategias de control avanzadas como las predictivas basadas en modelos o las adaptativas.

La implementación de una estrategia o de otra depende de varios parámetros, entre los cuales se pueden destacar los siguientes:

- Disponibilidad de los sensores u observadores necesarios para evaluar la variable a controlar.

- Naturaleza y robustez de los actuadores. Diferenciando entre elementos que permiten un control en bucle abierto, en el caso de ser capaces de llevar el sistema siempre al mismo punto a partir de una acción de control determinada, y elementos que requieren re-alimentación o información del estado de la variable a controlar.

- Tiempo requerido para calcular la acción de control necesaria para llevar el sistema al punto deseado.

En el capítulo 2 se detallaran las técnicas de medida utilizadas, sensores y actuadores en los motores diésel, así como la arquitectura y las principales funciones de la ECU. 
Control de Aire

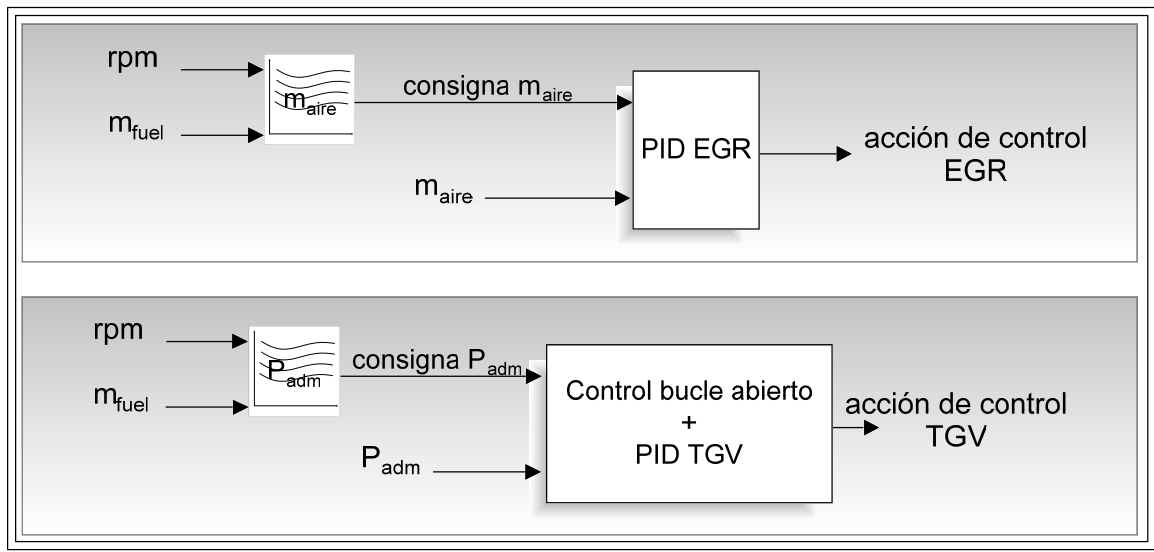

\section{Control de Combustible}

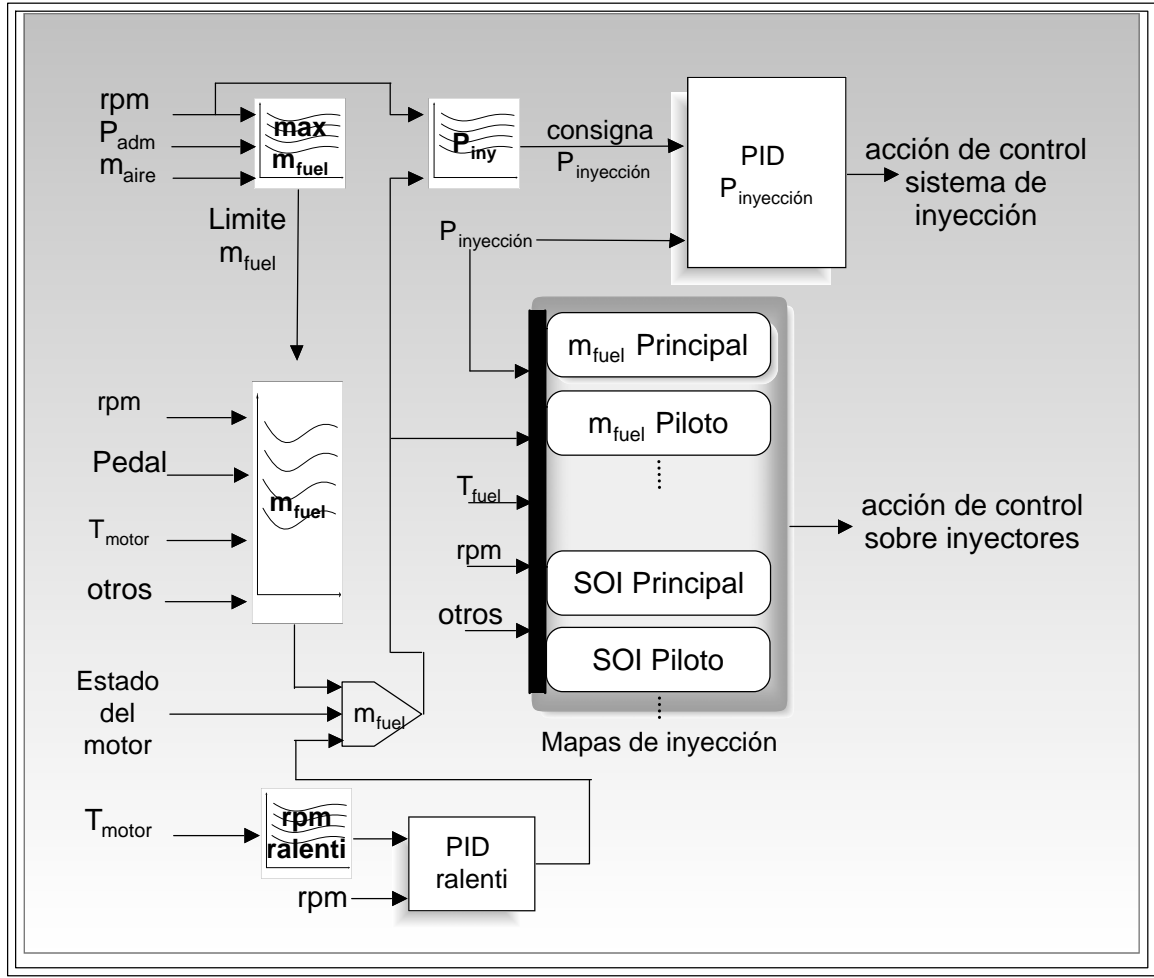

Figura 1.1: Sistemas de gestión del aire y de la inyección en motores diésel de última generación 


\subsubsection{Diagnóstico de la combustión}

Cuando se habla del diagnóstico de la combustión, se refiere a la estimación de parámetros relacionados con el proceso inyección-combustión desarrollado dentro del cilindro y que aportan información de lo que ocurre a lo largo de un ciclo termodinámico del motor después de producirse la renovación de la carga.

La energía química generada a partir de la mezcla aire-combustible dentro del cilindro es convertida en trabajo mecánico (transmitido al pistón) y calor mediante el proceso de la combustión. Maximizar el trabajo mecánico obtenido por esa conversión es equivalente a maximizar la eficiencia del motor. Esto fue, desde finales del siglo XVII, el tema de varios estudios y el objetivo principal de los desarrolladores de motores. Entonces, nació la necesidad de saber, en motores reales, el balance energético a lo largo de un ciclo termodinámico. El trazador del diagrama indicador fue la primera herramienta que permitía aportar información básica sobre lo que pasaba dentro del cilindro.

Hoy en día, la optimización de los motores de combustión interna de última generación es casi imposible sin el adecuado y detallado conocimiento de los fenómenos que trascurren dentro del cilindro. No sólo a nivel del trabajo mecánico producido, sino también a nivel de cómo se desarrolla exactamente la combustión real, cuanta energía realmente es aprovechada y qué características tienen los gases producidos por la combustión.

El indicador más importante de la forma en que se produce la combustión es el llamado ley de liberación de calor, que refleja la cantidad de calor liberada en cada instante, de donde se puede deducir parámetros de la combustión de gran interés como las fracciones quemadas en función del ángulo de giro, retraso de la combustión y, en definitiva, la calidad de la misma. Dicha calidad, permite establecer relaciones de causaefecto útiles para comprender los fenómenos implicados en la combustión, mejorar la eficiencia de los motores, reducir los contaminantes y en definitiva alargar la vida útil de los motores.

La geometría del cilindro, las características del flujo de aire dentro del cilindro, el estado y la composición de los gases dentro del cilindro son factores principales que afectan la calidad de la combustión. Por este motivo, cualquier desarrollo en motores está justificado por la mejora en la calidad de la combustión analizada mediante herramientas de diagnóstico de la combustión.

Los investigadores coinciden en las grandes virtudes del diagnóstico de la combustión. Las relaciones causa-efecto entre la ley de liberación de calor y geometría de cilindro, consumo y forma de introducir el combustible, emisiones y ruido están ampliamente documentadas en los foros científicos.

En cuanto a los procesos o las herramientas utilizadas para el diagnóstico de la combustión, se suele utilizar modelos termodinámicos basados principalmente en la medida de la presión en cámara. La complejidad de dichos modelos, depende del grado de precisión que se pretenda alcanzar.

Para el trabajo presentado en esta tesis se disponía del conocimiento existente en el grupo de investigación CMT-Motores Térmicos, basado en los trabajos previos de Macian [1], Tinaut [2], Oliver [3], Armas [4] y principalmente Martín [5] donde se hace 
un estudio detallado de los parámetros influyentes en el proceso de diagnóstico de la combustión.

En el capítulo 4 se procederá a resumir el proceso de diagnóstico de la combustión a partir de la presión en cámara elaborado y validado por el grupo de investigación e implementado en un ordenador bajo forma de un código comercial llamado CALMEC [6].

\subsection{Objetivos y Metodología}

\section{Objetivo:}

El objetivo principal de la presente tesis es aportar nuevas herramientas fiables para detección en tiempo real de parámetros de la combustión a partir únicamente de la medida de la señal de presión en cámara. Para ello se plantean los siguientes objetivos particulares:

- Definición de metodologías experimentales de puesta a punto de herramientas de medida y tratamiento digital de la señal de presión en cámara para estudios de la combustión. Dicha señal, es una variable sujeta a grandes ruidos, por lo tanto, se dedicará un esfuerzo especial a la optimización del uso de las herramientas matemáticas del filtrado de la señal para obtener medidas cuyo tratamiento aporta resultados precisos en el diagnóstico de la combustión. Paralelamente, la implementación de algoritmos en tiempo real precisa de sistemas electrónicos capaces de realizar la adquisición, el tratamiento digital y la actuación en tiempos razonables. Sistemas, para los cuales se pondrá de manifiesto las exigencias a las que están expuestos y se plantearan alternativas para reducir su coste, tanto a nivel computacional como económico.

- Parametrización rápida de fenómenos relacionados con la inyección-combustión que se producen dentro del cilindro mediante tratamiento digital de la señal en dominio temporal y en dominio de frecuencias. Es decir, relacionar los eventos detectados en la señal de la presión en cámara con algunos datos de la ley de liberación de calor especialmente la localización del inicio de la combustión (SoC), el final de la combustión (EoC), tiempo de retraso, máximo gradiente de liberación de calor, etc.

\section{Metodología:}

Primero se efectuara un estudio sobre las herramientas existentes de medida y control de motores de combustión interna alternativos (MCIA) actuales, así que las tendencias futuras. De esta forma, se identificarán los requisitos principales de las herramientas necesarias para futuras incorporaciones de estrategias de control basadas principalmente en el diagnóstico de la combustión.

Una vez se haya definido que variables hay que medir, con qué y bajo qué criterios, se establecerán los argumentos necesarios que justifican la naturaleza de la herramienta experimental con la cual se debe efectuar los ensayos experimentales para evaluar el proceso del diagnóstico de la combustión. 
Después, y tomando como base resultados experimentales de referencia, fiables y con las incertidumbres establecidas, se desarrollaran nuevas metodologías de diagnóstico de la combustión que pueden ser implementadas en las herramientas existentes de medida y control de MCIA actuales y se evaluarán comparando sus resultados con los resultados de referencia.

Por lo tanto, para empezar la tesis es conveniente estudiar las herramientas utilizadas para medida y control en motores diésel que aportarán información detallada sobre las características de los parámetros que posteriormente pueden ser utilizados bien para el diagnóstico de la combustión o bien para implementación de nuevas estrategias de control.

\subsection{Estructura de la tesis}

La estructura de la tesis será enumerada de la siguiente forma:

1. Se realizará en el capítulo 2 un estudio del estado del arte de las técnicas y las herramientas utilizadas en la gestion de los motores diésel de serie. Recalcando las prestaciones de las ECUs actuales, los sensores y actuadores que maneja y las estrategias de control ya implementadas en la mayoría de dichas ECUs. En el mismo capítulo, se recapitularán las tendencias futuras relacionadas con las técnicas de medida y control de motores de combustión interna alternativos.

2. La revisión bibliográfica será completada en el capítulo 3 donde se detallará el caso de la medida y el uso de la presión en cámara. En el mismo capítulo, se definirán los requisitos para la medida de presión en cilindro, identificando los parámetros claves y aportando alternativas de una conveniente instrumentación de medida según el caso de aplicación.

3. En el capítulo 4 se presentarán las herramientas experimentales que se utilizan para el desarrollo de la tesis. Haciendo un enfoque resumido sobre la herramienta de diagnóstico de combustión a partir de la presión en cilindro usada para validar los resultados de la presente tesis, así como los requisitos impuestos por dicha herramienta como pueden ser las variables, precisiones y la necesidad del tratamiento de señales.

4. En el capítulo 5 se aportarán nuevos criterios del tratamiento de la señal de presión en cámara que permiten obtener una medida adecuada para su uso en el diagnóstico de la combustión. Principalmente, criterios y metodologías de filtrado que se deben aplicar a la señal de presión en cámara para disminuir las ambigüedades en el diagnóstico de la combustión en tiempo real. Dicho capítulo será cerrado con la propuesta de una alternativa para la medida de la señal de la derivada de la presión en cámara necesaria para el desarrollo de las metodologías que serán desarrolladas en el capítulo 6 .

5. En el capítulo 6 se presentarán las nuevas metodologías propuestas para parametrización de la combustión basadas en el tratamiento digital de la señal de la derivada de la presión en cámara. Así mismo, se discutirán los resultados 
experimentales obtenidos por cada metodología propuesta para determinar la capacidad, las ventajas y los inconvenientes de cada metodología.

6. Para finalizar, en el capítulo 7 se realizará una síntesis de las principales aportaciones y conclusiones de la tesis. Así mismo, se indicarán posibles trabajos futuros que pueden dar continuidad a la presente tesis, incidiendo en aquellos aspectos que del desarrollo del presente trabajo se haya concluido que pudieran ser abordados con mayor profundidad. 



\section{Bibliografía del capítulo}

[1] V. Macian. Contribución al estudio de la influencia del movimiento del ruido sobre la dispersión cíclica y su caracterización. Tesis Doctoral, Universidad Politécnica de Valencia, 1984.

[2] F.V. Tinaut. Contribución al estudio del proceso de combustión en motores de encendido por compresión de inyección directa. Tesis Doctoral, Universidad Politécnica de Valencia, 1986.

[3] E. Oliver. Estudio de sistemas de adquisición y tratamiento de datos en motores de combustión interna alternativos. Tesis Doctoral, Universidad Politécnica de Valencia, Valencia, 1991.

[4] O. Armas. Diagnostico experimental del proceso de combustión en motores Diesel de inyección directa. Tesis Doctoral, Universidad Politécnica de Valencia, Valencia, Spain, 1998.

[5] J. Martín. Aportación al diagnóstico de la combustión en motores Diesel de inyección directa. Tesis Doctoral, Universidad Politécnica de Valencia, 2007.

[6] F. Payri, S. Molina, J. Martín, y O. Armas. "Influence of measurement errors and estimated parameters on combustion diagnosis". Applied Thermal Engineering, 26:226-236, 2006. 

Capítulo 2

\section{Técnicas utilizadas para medida y control de MCIA}




\section{Índice}

2.1. Introducción $\ldots \ldots \ldots \ldots \ldots \ldots \ldots \ldots \ldots \ldots$

2.2. Plataforma $\ldots \ldots \ldots \ldots \ldots \ldots \ldots \ldots \ldots \ldots \ldots$

2.2.1. El microcontrolador . . . . . . . . . . . . . . . . 21

2.2.2. Acondicionamiento de señales . . . . . . . . . . . . . . . 22

2.2.3. Limitaciones . . . . . . . . . . . . . . . . 22

2.3. Variables a medir y sensores $\ldots \ldots \ldots \ldots \ldots \ldots \ldots 23$

2.3.1. Sensores de medida de temperaturas . . . . . . . . . . . 25

2.3.2. Sensores de medida de presión . . . . . . . . . . . . 26

2.3.3. Sensor de medida de régimen de giro . . . . . . . . . . 26

2.3.4. Sensor de caudal de masa de aire . . . . . . . . . . . . . . 27

2.3.5. Sensor de concentración de oxígeno . . . . . . . . . . . 27

2.3.6. Sensores de posición o desplazamiento . . . . . . . . . . 27

2.3.7. Otros sensores y tendencias . . . . . . . . . . . . 28

2.4. Actuadores . . . . . . . . . . . . . . . . . 29

2.5. Estrategias de control $\ldots \ldots \ldots \ldots \ldots \ldots \ldots \ldots$

2.6. Las tendencias futuras $\ldots \ldots \ldots \ldots \ldots \ldots \ldots \ldots \ldots$

Bibliografía del capítulo . . . . . . . . . . . . . . . . . . . 34 


\subsection{Introducción}

Las últimas cuatro décadas han sido testigo del vertiginoso aumento del número e importancia de aplicaciones electrónicas en los sistemas de vehículos.

En 1974, un grupo de ingenieros pioneros reunidos en la primera conferencia de la “Convergence Transportation Electronics Association”, identificaron 55 áreas probables donde la electrónica podría aplicarse y tener un papel decisivo en el desarrollo de los automóviles.

En 1982, 37 de estas tecnologías, incluyendo subsistemas electrónicos, estaban ya implementadas. Se pueden mencionar los sistemas de inyección electrónica, consumo de combustible, anti-bloqueo de ABS, bloqueo automático de puertas, control automático de crucero, diagnóstico de abordo y avisadores de mantenimiento entre otros.

En 1993, el $93 \%$ de las tecnologías eran una realidad y equipaban a vehículos de media y alta gama. Asimismo, se detectaron nuevas áreas donde la tecnología electrónica podía aplicarse y que aquellos ingenieros pioneros no hubieron podido imaginar. En este sentido, se pueden mencionar los sistemas de control de tracción, cuatro ruedas directrices, conductos de inducción de aire controlados electrónicamente, sintetizadores y reconocimiento de voz y control de carga eléctrica entre otros.

La aplicación de la electrónica en el automóvil está íntimamente relacionada con el desarrollo y la evolución de componentes electrónicos, principalmente los circuitos integrados.

A finales de los años 70, los circuitos integrados digitales ampliaron la capacidad funcional de los sistemas electrónicos, cuando fueron capaces de subsanar los problemas de temperatura, compatibilidad electromagnética y de sensibilidad ambiental que manifestaban los circuitos integrados analógicos.

En los años 80, los microprocesadores de 4 y 8 bits evolucionaron hacia los de 16 bits, permitiendo el desarrollo y la optimización de los sistemas de inyección electrónica, gestión electrónica de motor, anti-bloqueo de freno (ABS), suspensión de dureza variable, navegación y guiado, dirección y climatización controlada electrónicamente.

Es en este momento cuando los diseñadores de automóviles comenzaron a pensar en automóviles como un sistema integrado y no como un conjunto de piezas y sistemas independientes. Esta filosofía de diseño incrementó sustancialmente el contenido de los sistema eléctricos -electrónicos. La arquitectura de los nuevos vehículos incluye los microprocesadores de 32 bits, redes multiplexadas estandarizadas, sistemas de gestión de energía (EMS) con alternadores de 42 voltios, convertidores de 42-12 voltios y módulos de gestión de energía y carga. Términos como automatización e integración del vehículo se mencionan cada vez con mayor frecuencia.

Hoy en día, el mercado de los microcontroladores mueve anualmente alrededor de 11.5 billones de euros y estando más del $20 \%$ de la producción dirigida al sector de la automoción. 
En definitiva, los microprocesadores son el corazón operacional de las unidades de control electrónico (ECU) de todos los sistemas del automóvil controlados electrónicamente. Sin embargo, no podrían ejercer toda su función sin el aporte tecnológico de los diferentes sensores y actuadores inteligentes que completan la plataforma de control electrónico de vehículos. Esta plataforma se puede esquematizar en la figura (2.1).

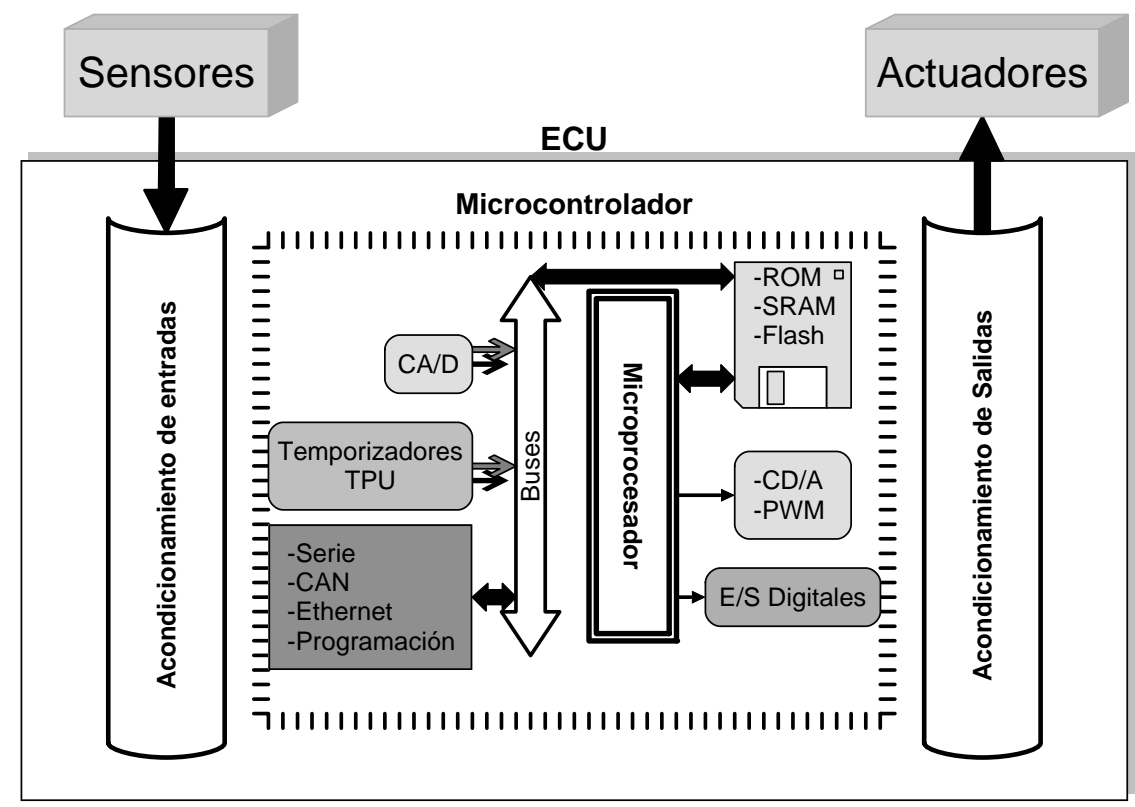

Figura 2.1: Plataforma de gestión electrónica de motores

Conocer la fiabilidad al traducir una magnitud física en un valor digital (medida o adquisición), la potencia y la velocidad de tratamiento de los datos y la posibilidad de actuar sobre un sistema es fundamental para entender la viabilidad de implementar una estrategia de control en las plataformas de gestión electrónica de motores. Para ello, en los siguientes párrafos se detallarán las prestaciones y limitaciones de las plataformas actuales de gestión de motores, dejando para el final de este capítulo el análisis de las estrategias de control utilizadas y las que se pretende implementar en dichas plataformas.

Hay que señalar que las técnicas de medida y control implementadas por los fabricantes para la gestión de los motores comerciales están restringidas por su coste económico. Esto explica que no siempre se usan los sensores más precisos, los actuadores más rápidos o las estrategias de control más avanzadas. 


\subsection{Plataforma}

\subsubsection{El microcontrolador}

Como se comentó anteriormente, el microcontrolador es el corazón de las unidades de control electrónico actuales. Es el elemento encargado de las operaciones matemáticas y de la gestión de datos que recibe de los diferentes elementos que completan el microcontrolador. Dichos elementos, tal y como se describirán a continuación, son: el microprocesador, las memorias, los convertidores, temporizadores e interfaces de comunicación con otros microcontroladores (figura (2.1)).

El microprocesador: la unidad de procesamiento de datos donde se manejan los registros binarios. Se encarga de gestionar la memoria de instrucciones, recibir la instrucción en curso, su decodificación y la ejecución de la operación que implica dicha instrucción, así como de la búsqueda de los operandos y el almacenamiento del resultado. Sus principales características son el número de instrucciones que puede procesar por segundo y la precisión de los datos a manejar. Actualmente, los microcontroladores de las ECUs alcanzan una frecuencia de procesamiento de instrucciones de $132 \mathrm{MHz}$ con una resolución de 32 bits.

Las memorias de programa y de datos son registros binarios donde se guardan las instrucciones a ejecutar en cada instante, así como los valores de las constantes y variables con que se ejecutan las instrucciones. La diferencia entre un tipo de memoria y otro es básicamente la velocidad de acceso a los registros, el tamaño de registros y la volatilidad de los registros.

Los convertidores analógico-digitales (CA/D) permiten transformar un voltaje en un valor binario cuya longitud afecta a la resolución de las medidas. Generalmente los microcontroladores de las ECUs actuales disponen de hasta 40 CA/D de 12 bits. Eso implica la posibilidad de la lectura de hasta 40 variables físicas mediante sensores correspondientes con una frecuencia de muestreo de hasta 400000 muestras por segundo.

Los temporizadores se utilizan para generar y evaluar eventos temporales con una precisión temporal del orden de nanosegundos. Los microcontroladores de las ECUs actuales disponen de unidades autónomas para el control de procesos temporales llamadas TPUs “time processor units”, cuya aplicación principal es la medida de régimen de giro instantáneo de los motores.

Interfaces con periféricos: con el objetivo de comunicarse con los periféricos externos, los microprocesadores disponen de una serie de interfaces llamados puertos, que comunican con protocolos normalizados con cualquier tipo de periférico externo. Entre ellos encontramos:

- Puertos serie: llamados también adaptadores de comunicación serie asíncrona (UART) o síncrona y asíncrona (USART).

- Puertos CAN ("Controller Area Network"): para permitir la adaptación con redes de conexión multiplexada, es decir mediante el mismo puerto se puede conectar un gran número de interfaces. Este tipo de puertos es el más utilizado por las ECUs actuales. No sólo se usa para comunicar con los diferentes sistemas de gestión 
electrónica de los vehículos actuales, sino que últimamente también una amplia gama de sensores aportan los datos a la ECU mediante este interfaz.

Convertidores digital-analógicos (CD/A): traducen los valores binarios del microprocesador en voltajes para gestionar diferentes actuadores. Las ECUs actuales pueden manejar hasta 24 actuadores.

Entradas y salidas digitales: corresponden a valores binarios de un bit ( $\begin{aligned} & 0 \\ & \text { o }\end{aligned}$ 1).

Moduladores de anchura de impulsos (PWM): convierten valores digitales en señales cuadradas con frecuencia definida y duración de pulso variable. Su principal aplicación es el control de las válvulas que requieren este tipo de señales eléctricas (Válvula EGR, Válvula de estrangulamiento de admisión, etc.).

\subsubsection{Acondicionamiento de señales}

Debido a la dificultad de implementar filtros digitales, el tamaño de las ECUs sigue siendo considerable a causa de la necesidad de etapas de acondicionamiento de señales electrónicas.

Existen dos tipos de acondicionamiento de señales de entrada:

- Estabilizadores de señales de alimentación.

- Acondicionadores de señales de sensores: filtros analógicos, amplificadores y circuitos de protección.

En cuando a las señales de salida encontramos:

- Circuitos de protección y aislamiento.

- Amplificadores como la etapa de potencia que aporta las tensiones y las corrientes necesarias para manejar los inyectores.

\subsubsection{Limitaciones}

Desde el punto de vista de desarrollos futuros, la principal limitación de las unidades de control electrónica radica en la capacidad computacional de los microcontroladores. $\mathrm{Si}$ bien, estos dispositivos poseen una potencia de cálculo que excede a la necesaria para gestionar los vehículos actuales, algunas herramientas matemáticas que pueden ser necesarias para mejorar la gestión de los motores diésel requieren una potencia de cálculo muy elevada.

Algunas estrategias de gestión que necesitan cálculos complejos pueden influir considerablemente sobre las prestaciones de los microcontroladores. A modo de ejemplo, una transformada de Fourier de una serie de datos supone un coste computacional elevado, un motivo que explica la inexistencia de estrategias de control basadas en análisis en dominio frecuencia en las estrategias de control de las ECUs actuales o el uso de filtros digitales avanzados. 
Hay que anotar que la mayoría de las rutinas de control electrónico de los motores diésel se suelen ejecutar en un tiempo máximo de $10 \mathrm{~ms}$. Si este tiempo no se cumple (falta de determinismo) el funcionamiento del motor puede desestabilizarse.

Las características de un microcontrolador de la ECU quedan muy limitadas si se comparan con las de un ordenador personal corriente de hoy en día (2.5 Ghz 32 bits) . La forma de subsanar este hándicap es distribuir la gestión electrónica en varios subsistemas, dejando el control de sistemas auxiliares a otros microcontroladores fuera de la ECU y enviar por puerto CAN la información de los sistemas auxiliares a la ECU.

En cuanto al uso de la ECU para temas de investigación, la limitación principal es el número de variables al que se puede acceder. Como se detallará en el apartado 2.3, las variables a medir para la gestión actual de los motores no son muy elevadas, sin embargo, para las tareas de investigación se requieren más variables y medidas exigiendo mayor instrumentación y sistemas más potentes de adquisición de datos.

Para los campos de investigación, otra limitación que se debe tener en cuenta es la dificultad de manejar la información contenida en las ECUs. Los fabricantes protegen la información y los accesos a las estrategias de control programadas, imposibilitando en muchas ocasiones el desarrollo y/o la validación de nuevas metodologías.

\subsection{Variables a medir y sensores}

La tabla (2.1) presenta un resumen de las variables a medir y los sensores que se encargan de dichas medidas para la gestión de los motores diésel common-raíl turbo alimentados de última generación. 


\begin{tabular}{|c|c|c|}
\hline & Variable & Tipo de sensor \\
\hline \multirow{9}{*}{$\begin{array}{l}\text { Sistema de } \\
\text { admisión de } \\
\text { aire }\end{array}$} & $\begin{array}{l}\text { Temperatura } \\
\text { ambiente }\end{array}$ & Termorresistencia \\
\hline & Presión atmosférica & Sensor piezoresistivo \\
\hline & Masa de aire & Caudalímetro de hilo caliente \\
\hline & Presión de admisión & Sensor piezoresistivo \\
\hline & $\begin{array}{l}\text { Temperatura de } \\
\text { entrada de aire }\end{array}$ & Termorresistencia \\
\hline & $\begin{array}{l}\text { Posición Válvula } \\
\text { TGV }\end{array}$ & Sensor de posición resistivo \\
\hline & $\begin{array}{l}\text { Posición Válvula } \\
\text { EGR }\end{array}$ & Sensor de posición resistivo \\
\hline & $\begin{array}{l}\text { Posición válvula de } \\
\text { estrangulamiento }\end{array}$ & Sensor de posición resistivo \\
\hline & $\begin{array}{l}\text { Posición válvula } \\
\text { bypass }\end{array}$ & Sensor de posición resistivo \\
\hline \multirow{5}{*}{$\begin{array}{l}\text { Prestaciones } \\
\text { del motor }\end{array}$} & Posición del pedal & Sensor de posición resistivo \\
\hline & Régimen de giro & $\begin{array}{l}\text { - Sensor inductivo del cigueñal } \\
\text { - Sensor inductivo del árbol de levas }\end{array}$ \\
\hline & $\begin{array}{l}\text { Temperatura del } \\
\text { refrigerante }\end{array}$ & Termorresistencia \\
\hline & Presión de aceite & Sensor piezoresistivo \\
\hline & $\begin{array}{l}\text { Temperatura del } \\
\text { combustible }\end{array}$ & Termorresistencia \\
\hline Inyección & Presión del raíl & Sensor piezoresistivo \\
\hline \multirow{3}{*}{ Emisiones } & $\begin{array}{l}\text { Concentración de } \\
\text { oxígeno }\end{array}$ & Sonda Lambda \\
\hline & $\begin{array}{l}\text { Gradiente de } \\
\text { presiones en la } \\
\text { trampa de partículas }\end{array}$ & $\begin{array}{l}\text { - Sensor piezoresistivo salida del filtro } \\
\text { de partículas } \\
\text { - Sensor piezoresistivo entrada del filtro } \\
\text { de partículas }\end{array}$ \\
\hline & $\begin{array}{l}\text { Temperaturas en el } \\
\text { escape }\end{array}$ & Termopares \\
\hline
\end{tabular}

Tabla 2.1: Variables a medir y sensores 


\subsubsection{Sensores de medida de temperaturas}

Existen dos rangos principales de medida:

\section{Temperaturas bajas entre -40 y $170^{\circ} \mathrm{C}$}

En este rango, los sensores más utilizados están basados en resistencias, compuestas por materiales como Hierro, Zinc, Cobre, Cobalto, Magnesio y Níquel, de coeficiente térmico negativo (NTC) que disminuyen de forma logarítmica su resistencia al aumentar la temperatura. La no-linealidad de estos sensores suele ser compensada con circuitos acondicionadores, resultando al final una precisión de medida de alrededor de $3 \%$. Su coste reducido y estabilidad hace que este tipo de sensores sean los más utilizados para medir temperaturas de refrigerante del motor, del combustible y ambiental.

\section{Temperaturas altas hasta $750^{\circ} \mathrm{C}$}

Para este rango se emplean dos tecnologías:

- Termorresistencia, se basa en la característica que tienen ciertos materiales, como el Cobre, el Níquel o el Platino, de aumentar su resistencia eléctrica al incrementar la temperatura. Las termorresistencias de platino (Pt100, Pt200) son las más comunes. La adecuada sensibilidad a las variaciones de temperaturas, linealidad, vida útil y su alta precisión presentan las mayores ventajas. La limitación de este tipo de sensores radica en el elevado coste, gran tamaño, lentitud de respuesta (casi 2 seg), auto calentamiento y la necesidad de un puente de resistencias alimentado para poder obtener una señal analógica proporcional a la temperatura medida.

- Termopares, menos precisos $\left( \pm 1.5^{\circ} \mathrm{C}\right)$ pero con un rango amplio de medidas $(-200$ a $2000^{\circ} \mathrm{C}$ ), económicos, rápidos y pequeños. Presentan la tecnología más utilizada para medida de altas temperaturas. Utilizan el efecto físico (efecto Seebeck) de la tensión generada entre conductores de diferentes materiales cuando su punto de soldadura (punto caliente) se encuentra a una temperatura distinta a la de los extremos abiertos de los conductores (junta fría). El voltaje generado es proporcional a la diferencia de temperatura entre el lado caliente y frío. Por lo tanto, los termopares proporcionan una lectura de temperatura relativa. Para obtener una medida exacta de temperatura absoluta, se requiere un conocimiento exacto de la temperatura de referencia (en la junta fría) y recompensarla para obtener los voltios proporcionales a la temperatura absoluta. La señal en un voltaje típica de $60 \mathrm{mV} / 1000{ }^{\circ} \mathrm{C}$, suele ser amplificada y adaptada para las entradas analógicas de la ECU.

El bajo coste de los termopares y el tiempo de respuesta adecuado (aproximadamente $0.3 \mathrm{seg}$ ) les hace óptimos para medida de variaciones de temperaturas rápidas en gases. Se encuetran principalmente implementados para la medida de temperaturas en el escape. 


\subsubsection{Sensores de medida de presión}

Para la gestión de los motores diésel, las ECUs requieren una serie de medidas de presiones generalmente en un rango de 0 a 10 bares a excepción de la presión de inyección o la presión en el common-raíl que ronda los 1800 bares en la actualidad.

Para ello, se emplean sensores piezoresistivos que se basan sobre el fenómeno de cambio de la resistencia eléctrica de ciertos materiales cuando se someten a presiones o sobre el fenómeno de cambio de la resistencia de conductores (galgas extensiométricas) al cambiar de longitud o de sección. Esta última tecnología es la más extendida puesto que la mayoría de los sensores piezoresistivos, de los usados en el campo de automoción, están compuestos de unas galgas extensiométricas colocadas sobre una membrana que al deformarse cambia la resistencia de las mismas y aportan una lectura proporcional a la presión absoluta.

La sensibilidad de estos sensores viene determinada por la naturaleza, longitud y diámetro del material utilizado por una parte y por la flexibilidad de la membrana por otra. En general, dicha sensibilidad es óptima para medir presiones estáticas en procesos con poca variabilidad térmica. Puesto que las dilataciones térmicas pueden provocar deformaciones permanentes, para condiciones extremas, este tipo de sensores requiere una estructura de membrana cuidadosamente diseñada y, en ocasiones, un circuito de refrigeración. Además, requieren una calibración periódica para contrarrestar el efecto de las deformaciones permanentes. Dicha calibración se encuentra actualmente integrada en las ECUs, alcanzando de esta forma una resolución media en la medida de la presión mediante estos sensores de $\pm 1 \%$ respeto al fondo de escala.

Aunque los sensores de medida de presión son altamente costosos, el gran aporte de información que permiten recompensar dicho coste. De hecho, después de implementar a finales de los años 80 el sensor de presión de admisión, cada vez se incluyen más sensores de presión para gestionar los motores diésel como es el caso de los sensores de presión a la entrada y salida de los catalizadores para regular los contaminantes.

\subsubsection{Sensor de medida de régimen de giro}

Se trata de un sensor inductivo, implementado sobre el cigüeñal, capaz de detectar el paso de los dientes. Generalmente la rueda dentada del cigüeñal está compuesta de 58 dientes más un hueco correspondiente a dos dientes que permite tener una referencia respecto al PMS de un cilindro del motor. De esta forma, la resolución angular máxima que permite aportar la rueda dentada es de $6^{\circ}$.

La señal que aporta este sensor es de alto voltaje y semejante a una senoidal. Una etapa de acondicionamiento de dicha señal, permite obtener una señal de pulsos (TTL) que será ampliada mediante la unidad de procesos temporales (TPU) del microprocesador de la ECU para obtener el régimen de giro instantáneo. 


\subsubsection{Sensor de caudal de masa de aire}

La tecnología más utilizada para este tipo de sensores es la llamada de hilo caliente. Consta de una resistencia eléctrica cuya temperatura se mantiene fija mediante un circuito de control electrónico. El aumento del caudal de aire produce un enfriamiento, por convección, más rápido de la resistencia, en consecuencia, el circuito de control aporta más corriente para llevar la resistencia a la temperatura de control. En definitiva, la acción de control aportada es proporcional al caudal de aire.

\subsubsection{Sensor de concentración de oxígeno}

Los sensores de oxígeno cumplen la función de generar una tensión de salida que corresponde al cociente entre la cantidad de oxígeno en los gases de escape respecto al O2 de la atmósfera.

Un dispositivo de cerámica compuesto de zirconio y recubierto por una película de platino compone este tipo de sensores. El dióxido de zirconio ( $\mathrm{ZrO} 2)$ es un sólido cristalino de alta conductividad iónica. Basándose en operación electro-química, la superficie interna del sensor, en contacto con el oxígeno del aire, por una parte y la superficie externa, en contacto con los gases de escape, forman dos electrodos cuya diferencia de potencial, definida por las ecuaciones de Nerst, es proporcional al factor Lambda $(\lambda)$ que relaciona la cantidad de aire suministrada y la requerida para una combustión completa.

Con el mismo principio de funcionamiento, los sensores lambda actuales disponen de calentadores para alcanzar rápidamente la temperatura de funcionamiento, estructuras laminares del dispositivo cerámico y cámaras cerradas que contienen el gas de referencia. Aun así, la tensión de salida del sensor no es lineal con respecto a la concentración de oxígeno, por lo que es necesario acondicionar el sensor. La ECU se encarga de controlar la temperatura y del acondicionamiento de la señal del sensor.

Nuevas tecnologías, como los sensores de oxígeno de banda ancha, permiten un mayor rango de medida y mayor resolución. Otras alternativas implementan sensores resistivos compuestos de dióxido de Titanio ( $\mathrm{TiO} 2)$ que cambian de resistencia en función de la presión parcial del oxígeno en los gases de escape.

\subsubsection{Sensores de posición o desplazamiento}

Estos sensores son ampliamente usados para medir posición de válvulas y de pedal del acelerador. Su principio de funcionamiento consiste en un potenciómetro desplazado mediante el mecanismo que se pretende medir.

Son sensores alimentados cuya salida es proporcional a la caída de tensión en la resistencia activa del potenciómetro. 


\subsubsection{Otros sensores y tendencias}

A continuación se indican otros sensores que, aunque no se encuentran de manera generalizada integrados en las estructuras de control de los motores, abren un gran abanico de posibilidades para la mejora del control de los motores.

\section{El sensor del levantamiento de aguja}

Incorporado en algunos inyectores, se basa en la velocidad del levantamiento de la aguja del inyector que induce un campo magnético en una bobina. La variación del flujo magnético inducido es proporcional a la tensión de salida inducida según la ley de Faraday. Dicha tensión es integrada mediante un circuito electrónico obteniendo una tensión proporcional al levantamiento de la aguja.

El transformador diferencial variable línea (LVDT) es otro principio para medida del desplazamiento de la aguja del inyector. Está constituido por una serie de inductancias y la aguja del inyector que compone el núcleo magnético. Una corriente alterna conocida es aplicada en una bobina primaria, dicha corriente produce un campo magnético variable alrededor del núcleo, que a su vez induce un voltaje en la bobina secundaria. La diferencia de potencial en la bobina secundaria será proporcional al movimiento del núcleo y por lo tanto al de la aguja. Este sistema se utiliza ampliamente debido a su gran resolución, alta linealidad y rápida respuesta.

Hoy en día, el uso de estos sensores sigue estando limitado al diagnóstico de fallos de inyección. Sin embargo, la tendencia futura es usar este tipo de sensores para retroalimentar el bucle de control de cantidad de combustible inyectada.

\section{El sensor de NOx}

Básicamente consiste en dos sensores de concentración de oxígeno donde el primer sensor mide la concentración de oxígeno en los gases de escape que en una segunda etapa se calientan a temperaturas suficientemente altas para reproducir la reacción química que descompone las moléculas de $\mathrm{NO}\left(2 \mathrm{NO} \rightarrow \mathrm{N}_{2}+\mathrm{O}_{2}\right)$. La diferencia entre las concentraciones de oxígeno aportada por los dos sensores es proporcional a la concentración de $N O$ en los gases de escape. Para obtener la concentración de los $N O$ x es necesario hacer un ajuste al valor medido por este tipo de sensores.

Todo indica que este tipo de sensores van a ser tenidos muy en cuenta para los fabricantes de motores con objeto de superar la normativa EURO 6. Dichos sensores presentan una buena precisión. El único inconveniente a día de hoy radica en su tiempo de respuesta (unos $300 \mathrm{~ms}$ ). 


\section{El sensor de presión en cámara}

Dada la relevancia de estos sensores en esta tesis, en el capítulo 3, se detallara ampliamente este tipo de sensores y su potencial para la gestión de motores diésel.

\subsection{Actuadores}

Básicamente, la ECU asegura el control electrónico del motor mediante los siguientes actuadores:

- Reguladores de la presión del combustible como la válvula de descarga para la regulación presión del common-raíl o las bombas inyectoras.

- Reguladores de la cantidad de combustible a inyectar como los actuadores de los inyectores (duración de la inyección).

- Reguladores de masa de aire como electroválvula de recirculación de los gases de escape (EGR), válvula de admisión y electroválvula del turbocompresor con geometría variable.

- Reguladores de temperatura de aire de admisión (calentadores).

- Reguladores de temperatura de motor.

Las acciones de control son principalmente de tres tipos:

- Tensiones continuas en un rango de 0 a $12 \mathrm{~V}$.

- Señales eléctricas de ancho de pulso modulable (PWM), en forma de pulsos de frecuencia constante y duración del estado alto $(12 \mathrm{~V})$ proporcional al valor de la acción de control. La energía de dicha señal es convertida mediante un solenoide en un campo magnético uniforme capaz de mover un vástago que permite el movimiento de válvulas.

- Conexión y desconexión alimentando o desactivando un actuador electrónico, bien mediante señales digitales (Relés) que provienen de la ECU, o bien mediante pulsos convertidos mediante una etapa de potencia que eleva la acción de control a los valores de corriente y tensión necesarios para activar elementos que requieren alta potencia (inyectores de bobina u inyectores piezoeléctricos).

Los actuadores que regulan la masa de aire y que requieren una señal PWM son relativamente lentos. Sus tiempos de respuesta suelen tardar alrededor de $100 \mathrm{~ms}$. Eso no permite controlar la cantidad de masa de aire que se introduce en el cilindro cada vez que se renueve la carga (cada ciclo). Por lo tanto, la única variable controlada ciclo a ciclo y que influye directamente sobre las prestaciones del motor es el pulso de la inyección. 


\subsection{Estrategias de control}

Con el objetivo de optimizar el proceso de combustión en todo el rango de funcionamiento de un motor, la ECU calcula las cantidades de combustible y de aire necesarias en cada instante teniendo en cuenta un gran número de parámetros. Dichos parámetros no se corresponden siempre a medidas reales, al contrario, la gran mayoría son estimados mediante observadores y modelos empíricos fruto de un gran número de investigaciones.

Dichos modelos son gobernados por las ecuaciones físicas que rigen los procesos en estudio, siendo todas las variables factores físicos determinados. Sin embargo, desde el punto de vista de control, se consideran los procesos como cajas negras con unas condiciones de entorno (entradas y perturbaciones) conocidas y los modelos relacionan dichas condiciones de entorno con las salidas mediante funciones vectoriales de variables raramente correspondientes a factores físicos. De esta forma los modelos son catalogados como lineales y no lineales.

Los modelos lineales consideran el efecto de una combinación lineal de entradas y condiciones iniciales como una combinación lineal de sus efectos individuales sobre las salidas. Es decir, que una variable de salida de un modelo es una combinación lineal de la respuesta de varios submodelos independientes al variar las entradas del modelo de forma lineal. Dichos modelos y submodelos se clasifican como:

- Modelos basados en ecuaciones físicas [1,2], llamados también modelos heurísticos.

- Modelos empíricos basados en el comportamiento los subsistemas que, a partir de ensayos experimentales sobre el sistema, identifican la respuesta frente a cambios de la entrada utilizando herramientas matemáticas como pueden ser métodos de regresión o mínimos cuadrados.

El error de estos modelos respecto a los sistemas reales se minimiza mediante técnicas matemáticas basadas en la evaluación del error cuadrático, error de las variables y errores del modelo de predicción. Hendricks [3] detalla algunos modelos de control y tipos de observadores más usados por la comunidad científica para modelar el comportamiento de diferentes subsistemas de los motores. Algunos de estos algoritmos son implementados en las ECUs actuales con una mejora notable del comportamiento de los motores.

En consecuencia, además de las estrategias convencionales en bucle abierto y en bucle cerrado, las estrategias de control implementadas en las ECUs actuales son un híbrido de las convencionales. Especialmente, el control en cascada es ampliamente usado en la gestión de los motores diésel. Este tipo de control se compone de un lazo de control principal (p. ej. control de masa de aire mediante la válvula de EGR) donde se introducen varios lazos internos secundarios (p. ej. control de la presión de admisión mediante TGV, limitación de la cantidad de combustible, etc.) que dan lugar a una serie de consignas internas que mejoran la dinámica del control principal y atenúan el efecto de otras variables consideradas como perturbaciones.

De esta manera, las estrategias de control principales para el aporte del combustible y la gestión del aire se encuentran relacionadas entre sí. Como se podrá apreciar a 
continuación.

En un motor diésel common-raíl, la gestión del aporte del combustible se logra mediante tres parámetros: presión de raíl, duración e instante o punto de la inyección. Las consignas de la duración y la presión de inyección reflejan un modelado del tipo de inyector empleado, ya que la tasa del inyector, además de ser función de la presión y la duración de la inyección, depende de factores como la geometría del inyector, la dinámica del levantamiento de la aguja, las tensiones de la etapa de potencia y la densidad del combustible. El modelado, implementado en forma de tablas que deciden las consignas, tiene en cuenta todos los factores aneriores.

La presión de raíl se controla en bucle cerrado, generalmente mediante un algoritmo PID que a partir de la lectura de la presión de inyección, la corrige actuando sobre la válvula de descarga hasta alcanzar los valores de la consigna.

La duración y el punto de inyección tienen un efecto directo sobre la formación de los contaminantes y el ruido del motor [4-8]. La cantidad de combustible necesaria, determinada por la ECU, se suele repartir en varias inyecciones en función del régimen de giro. De esta manera se logra, mediante una o dos inyecciones piloto, disminuir el ruido y las emisiones de NOx del motor. Por medio de las altas presiones de la inyección principal (hasta 2000 bares en motores CR) se limita la formación de los NOx en la primera fase de la combustión y, mediante inyecciones posteriores a la principal, se logra controlar la temperatura de los gases de escape para etapas posteriores de tratamiento de los gases de escape y, por lo tanto,controlar la formación y las emisiones del hollín [9].

La determinación de la cantidad de combustible necesaria es función del estado del motor. Las estrategias más utilizadas para cada estado del motor son:

\section{Arranque}

Inyección de cantidad fija de combustible hasta superar un determinado valor de régimen de giro. Además de estrategias avanzadas de calentamiento y compensaciones para limitar contaminantes en caso de arranque en frío

\section{Ralentí}

Incorpora dos estrategias de control paralelas: la primera es el control en bucle abierto de la consigna de régimen de giro en función de la temperatura del motor y en caso de información disponible de los equipos auxiliares (alternador, aire acondicionado) se corrige el régimen de consigna. La segunda consta de un control PID que corrige la cantidad de combustible necesaria para alcanzar el régimen de giro consigna. 


\section{Funcionamiento normal}

En función del régimen de giro, posición de pedal, temperatura de combustible y del aire de admisión, la cantidad de combustible deseada se calcula mediante varios mapas. Dicha cantidad es limitada o compensada en función de varios parámetros, como por ejemplo:

- Limitación para no alcanzar el régimen de giro máximo del motor.

- Limitación debida a la temperatura del refrigerante o del aceite del motor.

- Limitación para protección de los actuadores (inyectores susceptibles a inyecciones de duración muy larga).

- Limitación en función de la cantidad de emisiones estimada mediante sonda lambda o usando el gasto del aire o la presión de admisión (limitador de humos).

- Compensación en función de la presión ambiente.

- Compensación de la cantidad de combustible inyectada en cada cilindro en caso de existencia de información sobre el levantamiento de aguja.

- Compensación en función de la estimación del par demandado.

- Compensación en función de la riqueza de la combustión (uso de sonda Lambda).

Una vez decidida la cantidad de combustible a inyectar, la ECU determinará la masa de aire necesaria para una combustión óptima. En los motores turbo-alimentados, dicha masa de aire se consigue por dos vías principales:

- La turbina se encarga de transformar la energía de los gases de escape en energía mecánica transmitida al compresor, permitiendo aumentar la densidad del aire que entra en el cilindro. La ECU actúa sobre la turbina (mediante TGV o Swirl) permitiendo alcanzar mayores presiones de admisión y por lo tanto aumentar la densidad del aire introducido en el cilindro.

- La retroalimentación de los gases de escape introduce una porción de dichos gases de nuevo en la admisión. Esta forma presenta la mejor alternativa para disminuir los NOx. La ECU se encarga de controlar la apertura de la válvula de EGR para permitir mayor o menor paso de los gases de escape. Generalmente, los algoritmos de control del EGR se activan cuando se demanda poco par, es decir, en casos como el arranque en frió o aceleraciones bruscas el control del EGR es desactivado.

En los últimos años, a las dos anteriores vías se han sumado nuevos actuadores y sensores [10]. En general, se han implementado sensores de posición de las válvulas EGR y TGV, además de válvulas de estrangulamiento en la admisión y circuitos de refrigeración de los gases de escape.

Las consignas de masa de aire y la presión de admisión vienen dadas por tablas numéricas en función del régimen de giro y de la masa de combustible a inyectar. Las 
estrategias de control tradicionales usan algoritmos PID para el control de cada variable sin tener en cuenta el acoplamiento que existe entre los dos controles. Sin embargo, debido a los complejos sistemas actuales de control del aire de admisión, algoritmos de control en cascada, adaptativos o basados en modelos [11], son cada vez más frecuentes.

\subsection{Las tendencias futuras}

Con el constante objetivo de aumentar las prestaciones y disminuir los contaminantes de los motores diésel, las tendencias futuras pueden ser clasificadas principalmente en tres marcos de actuación:

1. Implementación de nuevas teorías de control basados en modelos, como los controles predictivos [11-13] y redes neuronales [14-17] que permiten alcanzar las consignas en tiempos óptimos además de estimar variables difíciles de medir.

2. Implementación de sistemas de tratamiento de gases de escape o actuadores más rápidos con el objetivo de hacer control ciclo a ciclo del motor diésel.

3. Implementación de nuevos sensores, principalmente los que puedan aportar información sobre la combustión en tiempo real.

A modo de ejemplo y dentro de la clasificación anterior, se destacan las siguientes vías en el campo de la investigación:

- Sistemas de tratamiento de los gases de escape: catalizadores "Diésel Oxidation Catalyst" (DOC), catalizadores selectivos "Selective Catalyst Reduction" (SCR) y filtros de partículas "Diésel Particulate Filter" (DFP)) [18],

- Control de temperatura de los gases de escape tomando en cuenta el efecto de los sistemas de tratamiento de los gases de escape en los bucles de control de la renovación de la masa de aire, especialmente con el uso del EGR baja presión [18].

- Control de masa de aire residual en los cilindros controlando la apertura y cierre de las válvulas mediante válvulas controladas "Variable Valve Timing"(VVT) [19,20].

- Nuevas estrategias de control del EGR [21] .

- Implementación de modelos más robustos [22] en el control de emisiones y sensores de NOx [23].

- Control de la combustión dirigido a nuevos motores de combustión interna no convencionales como los de carga homogénea en encendido por compresión "Homogeneous Charge Compression Ignition" (HCCI), los de carga premezclada en encendido por compresión "Premixed Charge Compression Ignition" (PCCI) o los de combustiones frías "Low Temperature Combustion" (LTC) [24-26].

- Control de conmutación entre diferentes nuevos conceptos de combustión en el mismo motor diésel. 
- Control basado en medida de la presión en cámara [27].

Al margen de las alternativas del post-tratamiento de los gases de escape, el aprovechamiento óptimo de la máxima energía del combustible inyectado y con la menor contaminación posible pasa forzosamente por un control del proceso de la combustión ciclo a ciclo. Por lo tanto, se puede afirmar que la mayoría de las mejoras previstas para el futuro de los motores diésel requieren de una forma directa o indirecta el diagnóstico de la combustión. Si los modelos matemáticos de la combustión pueden jugar un papel relevante en los futuros desarrollos, la medida de la presión en cámara es la vía más directa y fiable para un buen diagnóstico de la combustión y, por lo tanto, representa una de las estrategias más convenientes para la mejora de prestaciones y la reducción de los contaminantes de los motores de combustión interna alternativos. Sin embargo, es necesario realizar un especial esfuerzo con el objetivo de reducir el número de variables de ajustes de las estrategias de control y el tamaño de las medidas necesarias por una parte, y facilitar la implementación de las estrategias en los motores de serie, por otra [26]. 


\section{Bibliografía del capítulo}

[1] J. A. Cook y B. K. Powell. "Modeling of an internal combustion engine for control analysis”. Control Systems Magazine, IEEE, 8(4):20 -26, aug 1988.

[2] Elbert Hendricks y Spencer C. Sorenson. "Mean value modelling of spark ignition engines". SAE paper, (900616), 1990.

[3] E. Hendricks y J. Luther. "Model and observer based control of internal combustion engines". In Modeling, Emissions and Control in Automotive Engines, 2001.

[4] T. C. Tow, D. A. Pierpont, y R. D. Reitz. "Reducing particulate and nox emissions by using multiple injections in a heavy duty d.i. diesel engine". SAE paper, (940897), 1994.

[5] D. A. Nehmer y R. D. Reitz. "Measurement of the effect of injection rate and split injections on diesel engine soot and nox emissions". SAE paper, (940668), 1994.

[6] T. Minami, K. Takeuchi, y N. Shimazaki. "Reduction of diesel engine nox using pilot injection". SAE paper, (950611), 1995.

[7] D. A. Pierpont y R. D. Reitz. "Effects of injection pressure and nozzle geometry on d.i. diesel emissions and performance". SAE paper, (950604), 1995.

[8] G. Dober, S. Tullis, G. Greeves, N. Milovanovic, M. Hardy, y S. Zuelch. "The impact of injection strategies on emissions reduction and power output of future diesel engines". SAE paper, (2008-01-0941), 2008.

[9] A. Garcia Martinez. Estudio de los efectos de la post inyección sobre el proceso de combustión y la formación de hollín en motores diésel. Tesis Doctoral, Universidad Politécnica de Valencia, 2009.

[10] V. Nieuwstadt y J. Michiel. Method and system for controlling EGR rate in diesel engines. Patente, Estados Unidos, May 2004. 
[11] J.M. Luján, H. Climent, C. Guardiola, y J.V. García-Ortiz. "A comparison of different algorithms for boost pressure control in a heavy duty turbocharged diesel engine”. Proc. Inst. Mech. Eng. Part D, Journal of Automobile Eng., 220:629-640, 2007.

[12] S. Nakayama, T. Ibuki, H. Hosaki, y H. Tominaga. "An application of model based combustion control to transient cycle-by-cycle diesel combustion". SAE paper, (2008-01-1311), 2009.

[13] H. Jin, S. Choi, H. Jin-Kaist, y S. Choi-Kaist. "Sliding sector mode control with linear parameter varying diesel engine model". SAE paper, (2011-01-0696), 2011.

[14] T. Tsuchiya, S. Morishita, T. Enomoto, H. Sasaki, y M. Yoshimura. "Revolution control of generator diesel engine by neural network controller". SAE paper, (200301-0365), 2003.

[15] J. Galindo, J.M. Lujan., J.R. Serrano, y L. Hernández. “Combustion simulation of turbochargers hsdi diesel engines during transient operation using neuronal networks". Applied Thermal Engineering, 25:877-898, 2005.

[16] R. Johnsson. "Cylinder pressure reconstruction based on complex radial basis function networks from vibration and speed signals". Mechanical Systems and Signal Processing, 20:1923-1940, 2006.

[17] S. Saraswati y S. Chand. "Reconstruction of cylinder pressure for si engine using recurrent neural network". Neural Computing \&amp; Applications, 19:935-944, 2010. 10.1007/s00521-010-0420-6.

[18] T.V. Johnson. "Diesel emission control in review". SAE paper, 1(2008-01-0069):68$81,2008$.

[19] A. Ferrari, M. Chiodi, M. Bargende, y P. et al. Roberti. "Virtual set-up of a racing engine for the optimization of lap performance through a comprehensive enginevehicle-driver model". SAE paper, (2011-24-0141), 2011.

[20] A. Baccile, D. Ceccarini, L. Cheng, A. Iacoponi, T. Lake, A. Noble, y J. Stokes. "An innovative control system for a $2 / 4$ stroke switchable engine". SAE paper, (200701-1199), 2007.

[21] M. Yao, Q. Zhang, H. Liu, P. Zhang, Z. Lin, T. Lin, y J. Shen. "Diesel engine combustion control: Medium or heavy egr?”. SAE paper, (2010-01-1125), 2010.

[22] F. Payri, J. Arrègle, J.J. López, y E. Mocholí "Diesel nox modeling with a reduction mechanism for the initial nox coming from egr or re-entrained burned gases". SAE paper, (2008-01-1188), 2008.

[23] C. Quérel, O. Grondin, y C. Letellier. "State of the art and analysis of control oriented nox models". SAE paper, (2012-01-0723), 2012. 
[24] M. Hasegawa, Y. Shimasaki, S. Yamaguchi, M. Kobayashi, H. Sakamoto, N. Kitayama, y T. Kanda. "Study on ignition timing control for diesel engines using in-cylinder pressure sensor". SAE paper, (2006-01-0180), 2006.

[25] P. Tunestål y M. Lewander. "Model predictive control of partially premixed combustion". In Luigi Re, Frank Allgöwer, Luigi Glielmo, Carlos Guardiola, y Ilya Kolmanovsky, editors, Automotive Model Predictive Control, volume 402 of Lecture Notes in Control and Information Sciences, pages 171-181. Springer London, 2010.

[26] H. Xie, R. Stobart, P. Tunestal, L. Eriksson, Y. Huang, y P. Leteinturier. "Future engine control enabling environment friendly vehicle”. SAE paper, (2011-01-0697), 2011.

[27] D. Schiefer, R. Maennel, y W. Nardoni. "Advantages of diesel engine control using in-cylinder pressure information for closed loop control". SAE paper, (2003-010364):01-0364, 2003. 

Capítulo 3

\section{Utilizaciones específicas de la presión en cilindro}




\section{Índice}

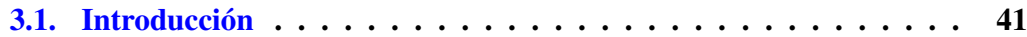

3.2. Uso de la presión en cámara . . . . . . . . . . . . 43

3.2.1. Presión máxima y ángulo de presión máxima. . . . . . . . . . 45

3.2.2. Presión media indicada . . . . . . . . . . . . . . . . 46

3.2.3. Diagrama $\log (p) / \log (V) \ldots \ldots \ldots \ldots \ldots$

3.2.4. Gradiente de presiones . . . . . . . . . . . . . . . . 47

3.2.5. Parámetros de la Combustión . . . . . . . . . . . . . . 47

3.3. Sistemas de medida de la Presión en cámara . . . . . . . . . . 49

3.3.1. Sensores y Acondicionadores . . . . . . . . . . . . . . . 49

3.3.1.1. Parámetros para elección de sensores de presión . . 49

3.3.1.2. Sensores de presión en cámara Piezorresistivos . . . 51

3.3.1.3. Sensores de presión en cámara ópticos . . . . . . . 52

3.3.1.4. Sensores de presión en cámara piezoeléctricos . . . 54

3.3.2. Características de la señal . . . . . . . . . . . . 56

3.3.3. Criterios de selección del sistema de medida . . . . . . . . 62

3.3.3.1. Resolución. . . . . . . . . . . . . . . . 63

3.3.3.2. Frecuencia de muestreo. . . . . . . . . . . . . 65

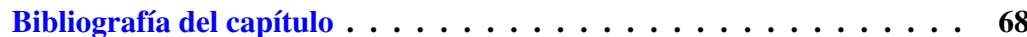




\subsection{Introducción}

Gran parte de la investigación está dirigida hacia la obtención de modelos fiables que, a partir del reducido número de sensores implementados en los motores, logren identificar variables no medibles y estimar parámetros de interés que puedan mejorar las prestaciones del motor. Estos modelos son usados en las ECUs actuales como generadores de consignas, es decir, generan valores de las variables físicas en los que se debería encontrar un sistema para que el comportamiento del motor sea óptimo. Los fabricantes se encargan de compactar los modelos y organizar las consignas, en forma de tablas y mapas, para reducir el coste computacional.

Desde el punto de vista físico, químico y termodinámico, algunos de los principales modelos van dirigidos hacia:

- El estudio de los procesos de la renovación de la carga. Se usan principalmente tres tipos de modelos [1-4]: modelos de flujo cuasi-estacionario, modelos de llenadovaciado y modelos de acción de ondas.

- El estudio del comportamiento hidrodinámico del sistema de inyección.

- El estudio de los procesos que ocurren dentro del cilindro $[2,5,6]$.

Los modelos obtenidos de los dos primeros tipos de estudios son los más implementados en el control de los motores de combustión interna alternativos de hoy en día. Los sensores, como se resumió en el capítulo anterior, necesarios para aportar la información requerida para dichos modelos están presentes en todos los motores de serie. Sin embargo, se carece de información relativa al proceso de combustión o de lo que ocurre en el seno del cilindro durante el ciclo termodinámico cerrado del motor (desde el cierre de las válvulas de admisión hasta la apertura de las válvulas de escape).

La presión en cilindro es la única variable factible de ser medida y que suministra información relativa al proceso de combustión. El conocimiento de dicha presión, así como de su evolución a lo largo de un ciclo de trabajo de motor, permite, no sólo obtener parámetros de diseño físico de elementos (cargas mecánicas), sino también parámetros relacionados con el proceso de combustión. La aplicación directa de las leyes de la termodinámica al volumen de control definido por el cilindro, pistón y culata, permite obtener la forma en la que se libera la energía en el proceso de combustión, una vez determinados ciertos parámetros (temperaturas de paredes, masas de aire y combustible, etc.) y siempre que se conozca de alguna manera el valor de la presión en cámara en cada instante del ciclo de trabajo del motor. Además, el conocimiento de dicha presión permite calcular de forma inmediata los trabajos indicados, de bombeo del motor, así como aportar una cantidad de información valiosa sobre el proceso de la combustión como se detallará más adelante.

Temperaturas que alcanzan $\operatorname{los} 1600^{\circ} \mathrm{C}$, gases corrosivos, partículas sólidas, fenómenos resonantes y sobre todo presiones que pueden variar de 1 a 250 bares en menos de $2 \mathrm{~ms}$ (125000 bar/seg), hacen que la medida de la presión en cámara sea altamente complicada. Además, se necesita hacer uso de sensores intrusivos para que el sensor este directamente en contacto con el gas encerrado dentro del cilindro. Eso presenta las mayores dificultades 


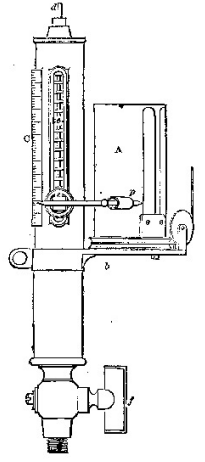

1769

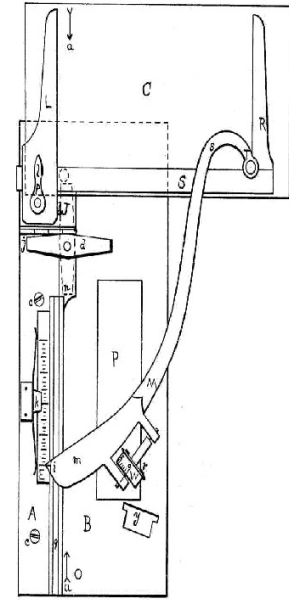

1882

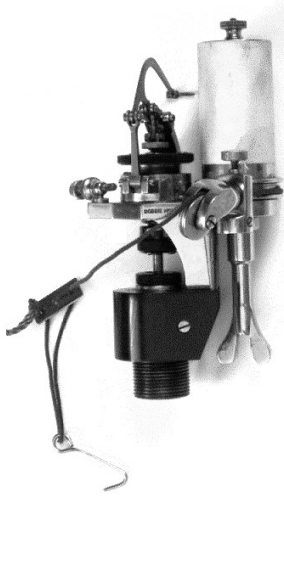

1903

Figura 3.1: Trazadores del diagrama indicador

puesto que dicho sensor tendrá que soportar condiciones dentro del cilindro altamente exigentes.

Desde finales del siglo XVII, los dispositivos de medida de la presión en el cilindro han sido objeto de gran cantidad de estudios. Uno de los primeros dispositivos fue inventado en 1769 por James Watt para trazar el diagrama indicador $p$ - $V$ en la cámara de su máquina de vapor. Consiste en un sistema mecánico accionado por el propio movimiento del pistón (eje de volumen) y por la presión en la cámara (eje de presión)(figura(3.1)). Este dispositivo fue mejorado a lo largo del siglo XVIII, obteniéndose así dispositivos mecánicos para el trazado del diagrama $p$ - $V$ para desarrollo de motores.

Los dispositivos de medida de la presión en la cámara han evolucionado desde entonces pasando por indicadores mecánicos de presión media y máxima, dispositivos electromecánicos, como los indicadores estroboscopios, hasta los modernos sistemas de adquisición basados en el uso de cristales piezoeléctricos o foto-diodos para sensores ópticos $[7,8]$.

Existen otras alternativas que usan básicamente sensores no intrusivos como acelerómetros que miden las vibraciones del motor, ofreciendo una señal que puede construirse de forma digital para obtener a partir de la misma la mayor información de la presión dentro del cilindro [9]. Estas alternativas dan mucha menor precisión y señales con mayor nivel de ruido.

Independientemente del tipo de sensor utilizado, las tecnologías modernas se basan en traducir la variación de la presión en señales eléctricas. Estas señales se someten a las siguientes etapas antes de ser registradas: 
- Etapa de amplificación: la magnitud de las señales eléctricas aportada por los sensores es muy baja y requiere ser amplificada para poder ser registrada.

- Etapa de acondicionamiento: en esta fase se elimina el ruido eléctrico tratando de no alterar la información contenida en la señal. Si las magnitudes de la señal lo permiten, esta etapa debe preceder a la etapa de amplificación con el fin de no amplificar el ruido.

Con el fin de aclarar la importancia de la presión en cámara, se partirá de un resumen de las principales aplicaciones en las que se suele utilizar esta variable, haciendo un especial énfasis sobre el impacto de la calidad de la medida sobre la información extraída mediante el uso de dicha presión.

En los siguientes apartados se detallaran los requisitos para un sistema de adquisición de datos para medida de la presión en cámara. Se partirá de la metodología para seleccionar un sensor adecuado de presión en cámara, se explicaran los métodos de acondicionamiento de la señal fuente y se dará luz a las características eléctricas de la señal eléctrica final. Estos aspectos pueden determinar los requisitos del sistema de medida óptimo y justificar su selección para la herramienta experimental del desarrollo de la tesis (capítulo 4).

\subsection{Uso de la presión en cámara}

El análisis de la presión en cámara puede aportar una gran cantidad de información mediante el análisis termodinámico de los datos registrados. La tabla (3.1) presenta algunas de las variables que se pueden calcular a partir de la presión en cámara así como parte de la información que puede obtenerse de dichas variables.

La información obtenida suele ser usada bien en la fase de diseño para determinar los limites y/o características del sistema, o bien para definir estado del sistema y determinar las acciones oportunas para mejorar el comportamiento del sistema (diagnóstico de fallos y control).

Como se comentó anteriormente, la calidad de la medida de la presión en cámara afecta a las variables calculadas y, por lo tanto, a la información obtenida. Para determinar los requisitos del sistema de medida se analizarán los efectos de los errores en la medida sobre las variables de interés. El sistema de medida debe anular. en todo lo posible, dichos efectos. 


\begin{tabular}{|c|c|}
\hline Variable & Información \\
\hline $\begin{array}{l}\text { Presión máxima y ángulo } \\
\text { de la presión máxima }\end{array}$ & $\begin{array}{l}\text { - Diagnóstico y tolerancia de fallos. } \\
\text { - Emplazamiento del PMS [10]. } \\
\text { - Cargas mecánicas [1,3]. } \\
\text { - Eficiencia. }\end{array}$ \\
\hline Diagrama $\log (p) / \log (V)$ & $\begin{array}{l}\text { - Estimación del exponente } \\
\text { politrópico. } \\
\text { - Estimación del PMS [11]. } \\
\text { - Estimación de inicio y final de la } \\
\text { combustión. } \\
\text { - Estimación de la masa } \\
\text { encerrada [12]. }\end{array}$ \\
\hline $\begin{array}{l}\text { Diagrama indicador y } \\
\text { presión media indicada }\end{array}$ & $\begin{array}{l}\text { - Prestaciones del motor, trabajo, } \\
\text { potencia, rendimiento, consumos } \\
\text { indicados, etc. }[1,3] \\
\text { - Perdidas mecánicas. } \\
\text { - Estabilidad y fallos de } \\
\text { combustión. } \\
\text { - Otros. }\end{array}$ \\
\hline Gradiente de presiones & $\begin{array}{l}\text { - Vibraciones } \\
\text { - Ruido de combustión }[13,14]\end{array}$ \\
\hline $\begin{array}{c}\text { Parámetros de la } \\
\text { Combustión } \\
{[1-4,10,15,16]}\end{array}$ & $\begin{array}{l}\text { - Ley de liberación de calor. } \\
\text { - Fracciones quemadas. } \\
\text { - Eficiencia de la combustión. } \\
\text { - Composición de gases. } \\
\text { - Temperaturas. } \\
\text { - Emisiones. } \\
\text { - Otros. }\end{array}$ \\
\hline
\end{tabular}

Tabla 3.1: Variables calculadas a partir de la presión en cámara 


\subsubsection{Presión máxima y ángulo de presión máxima.}

En general la presión máxima $\left(p_{\max }\right)$, como magnitud teórica, aporta información sobre los limites teóricos que tienen que soportar los elementos que constituyen el motor. Mediante la ecuación de los gases perfectos y aproximaciones termodinámicas se puede calcular la temperatura máxima $\left(T_{\max }\right)$ que se puede alcanzar dentro del cilindro, siempre que se disponga de información relativa a la transmisión de calor dentro de la cámara.

La presión máxima medida puede ser utilizada para diagnóstico de fallos como:

- Fallos por exceder la máxima presión teórica.

- Fallos de combustión: si se compara la $p_{\max }$ de un ciclo de motor en combustión con la de un ciclo de motor en arrastre, se puede establecer ratios para detectar ausencia o anomalías en la combustión.

- Fallos de medida: al estudiar la variación de $p_{\max }$ entre varios ciclos, se puede establecer un criterio para rechazar medidas donde la dispersión es muy alta [2].

El uso de $p_{\max }$ para control está más extendido en motores de encendido provocado que en motores diésel debido a la alta relación de compresión de estos últimos. Para los motores de encendido provocado son relevantes las publicaciones [17-21] entre otras, y que tratan principalmente de:

- Uso de $p_{\max }$ para limitar emisiones de NOx mediante estimación de $T_{\max }$ conociendo la masa atrapada en el cilindro y actuando sobre el inicio de la combustión [17].

- Optimización del gasto de combustible limitando la $p_{\max }$ [20].

- Control de la mezcla aire-combustible [19,21,22].

Sin embargo, la localización o el ángulo en el que se produce la $p_{\max }$ es más indicativo del comportamiento de la combustión. Entre otras aplicaciones en la literatura, y principalmente para motores de encendido provocado, se propone su uso para:

- Diagnóstico de inestabilidades y fallos de combustión a partir de la dispersión en la localización del ángulo de $p_{\max }$ [23].

- Control del inicio de la combustión [18].

- Control de emisiones, de mezcla aire-combustible y de recirculación de los gases de escape (EGR) $[17,19]$.

En estas aplicaciones y en otras, los requisitos de la medida de la magnitud de $p_{\max }$ son relativamente bajos. No se precisa una resolución elevada aunque se tiene que tener en cuento los efectos de la deriva térmica del sensor debido a las temperaturas elevadas que existen en el momento en el que se alcanza la $p_{\max }$. Es por esto que se aconseja el uso de sensores refrigerados para este ámbito de aplicaciones. En atención al uso que se va a hacer de ella, la precisión requerida para la determinación de la localización de la $p_{\max }$, se encuentra entorno a $1^{\circ}$. 


\subsubsection{Presión media indicada}

La pmi es el parámetro más significativo obtenido del diagrama de indicador. Permite caracterizar las prestaciones del motor y es representativo de otros parámetros indicados como el trabajo, potencia, rendimiento y consumo indicados.

Shimasaki [20] plantea el uso de la pmi para la estimación del par motor, control de la estabilidad de la combustión y control de EGR. Innumerables autores plantean el uso de este parámetro para el control de la combustión [21,24-26].

La mayoría de los estudios proponen usos de la pmi aplicado a motores de encendido provocado, siendo los métodos más usados aquellos que evalúan parámetros estadísticos de la variación de la pmi entre varios ciclos. Eso se puede explicar por la mayor dispersión cíclica existente en este tipo de motores. En estos casos de estudio, la resolución angular y la magnitud de la presión no son parámetros críticos, ya que solo se estudia la variación entre ciclos.

En cuanto a la precisión angular requerida para una determinación adecuada de la pmi, Brunt y Lucas [27] concluyen en su estudio que el cálculo de la pmi no es muy sensible a la resolución angular. Sin embargo, Martín [2] afirma que la pmi es muy sensible al desfase total, es decir, al correcto calado presión-volumen y que es mucho más critico que la resolución angular al que se muestrea la señal de la presión.

En general, hay que asegurar el correcto calado presión-volumen mediante ensayos específicos con muy alta precisión angular. Una vez determinado correctamente este parámetro, una resolución angular de $1^{\circ}$ es suficiente para una buena estimación de la pmi a partir de la presión en cámara medida [28].

La linealidad del sensor y una buena calibración es mucho más importante a la hora de calcular la pmi. Por otra parte, hay que tener en cuenta la corta deriva térmica de los sensores (short-term drift). Este fenómeno puede producir hasta un error del $9 \%$ en la estimación del pmi en el caso de utilizar sensores de calidad reducida [29].

\subsubsection{Diagrama $\log (p) / \log (V)$}

Este diagrama permite obtener el exponente politrópico durante la compresión, el cual es útil para otras aplicaciones como la estimación de la masa atrapada y de la masa de EGR [30]. Este diagrama se puede utilizar para estimar la posición del punto muerto superior (PMS) [11] y la dispersión de la relación de compresión entre los cilindros [12]. También puede ser utilizado para estimar los puntos de inicio y final de la combustión [31].

Los mismos requisitos de medida que se aplican cuando se utiliza la presión en cilindro para la estimación de la pmi se pueden aplicar para la construcción y el estudio del diagrama $\log (p) / \log (V)$. Principalmente, hay que tener en cuenta la deriva térmica de los sensores [32]. 


\subsubsection{Gradiente de presiones}

La derivada de la presión, como se detallará más adelante, se puede utilizar para detectar el inicio de la combustión y para estimar la velocidad máxima de combustión y su ubicación. La máxima $\Delta p / \Delta \alpha$ es normalmente utilizada como un estimador simple del ruido de combustión emitido por el motor [14].

Como se comentó anteriormente, para estudios de ruidos de combustión se precisa muy altas frecuencias de muestreo $(50 \mathrm{kHz})$. Es necesario en este caso que el muestreo sea temporal ya que la variación instantánea del régimen de giro genera errores en las frecuencias temporales calculadas a partir de las medidas realizadas mediante muestreo angular.

En los casos en los que la estimación de fenómenos que se producen durante la combustión (p.ej. detonación) se hace a partir del gradiente de presiones, la precisión en la estimación está directamente relacionada con la frecuencia de muestreo de la señal medida. A medida que se requiere mayor precisión se debe aumentar la frecuencia de muestreo.

\subsubsection{Parámetros de la Combustión}

Los métodos de diagnóstico de la combustión a partir de la presión en cámara analizan los procesos que ocurren en la cámara de combustión. Las técnicas utilizadas para esta exploración se llaman técnicas de diagnóstico de combustión, y permiten justificar algunos valores relativos a las prestaciones y emisiones del motor.

Estas técnicas son bidireccionales, es decir, se basan en la estimación de parámetros de la combustión (principalmente la ley de liberación de calor) a partir de señales experimentales medidas, o bien en la estimación de prestaciones del motor a partir de información relevante impuesta por el modelo de combustión o la ley de liberación de calor.

Estas técnicas complementarias, han sido clasificadas por Lapuerta [5]en:

- Técnicas experimentales que incluyen ensayos en motores reales o en modelos físicos simplificados.

- Técnicas de diagnóstico basadas en la medida e interpretación de alguna señal experimental.

- Técnicas teóricas basadas en el empleo de modelos de cálculo con diferentes grados de complejidad.

Fruto de la combinación de las dos primeras técnicas surgen los modelos termodinámicos de diagnóstico a partir de la presión que emplean las ecuaciones de los modelos termodinámicos teóricos (conservación de la masa y energía) sin imponer la ley de liberación de calor que es calculada a partir de la presión.

Si no se requiere alta precisión en el diagnóstico de la combustión se puede desestimar la transmisión de calor y considerar un comportamiento isoentrópico. La tasa 
de liberación de calor aparente puede ser calculada a partir del primer principio de la termodinámica mediante la ecuación:

$$
d F Q L=\frac{\gamma}{\gamma-1} \cdot p \cdot \frac{d V}{d t}+\frac{1}{\gamma-1} \cdot V \cdot \frac{d p}{d t}
$$

Sin embargo, cuando se requiere mayor precisión, como es el caso del desarrollo de la presente tesis, hay que recurrir a un conjunto de herramientas mucho más complejas mediante hipótesis que se acercan más a la realidad.

Como se ha comentado en la introducción de la presente tesis, para la elaboración de la misma, se ha partido del conocimiento existente en el grupo de investigación CMTMotores Térmicos, basado en los trabajos previos de Macian [33], Tinaut [34], Oliver [35], Armas [10] y principalmente Martín [2].

Sin ánimo de ser exhaustivos, y dada la importancia del modelo de diagnóstico de combustión que se va a utilizar para validar los resultados de esta tesis, en el capítulo de la descripción de las herramientas experimentales (capítulo 4) se procederá a describir los parámetros necesarios y los diferentes submodelos utilizados en el proceso de diagnóstico de la combustión a partir de la señal de presión. Además, para aclarar los diferentes submodelos, los detalles de cálculo y estimación de cada variable, así como la sensibilidad de los modelos y los diferentes ajustes de los modelos, se puede recurrir al trabajo de Martín [2].

De todos modos, los métodos más precisos de diagnóstico de combustión, usan la presión en cámara como entrada imprescindible. Varios estudios, como el propuesto por Payri [36] y Martín [4], revelan la sensibilidad de dichos métodos frente a la precisión en la medida de la presión en cámara. Martín [2] concluye su trabajo afirmando que:

- El desfase es crítico para el cálculo de las prestaciones del motor y la ley de liberación de calor.

- La incertidumbre de presión de referencia $\left(p_{r e f}\right)$ afecta considerablemente a la forma de la ley de liberación de calor.

Lapuerta y otros [37] afirman que el efecto de la calibración del sensor no es tan perjudicial como el del referenciado de la presión.

Debido a la gran cantidad de información que aporta la medida de la presión en cámara para el diagnóstico de combustión, la obtención de esta variable dota de un interés relevante al proceso de medida de la misma. Es decir, si se requiere obtener con alta precisión el inicio de la combustión, conviene medir con alta resolución angular. Pero si solamente interesa la ley de liberación de calor, una resolución angular baja sería conveniente.

Para estudios de investigación, tradicionalmente se usa una resolución angular de $0.5^{\circ}$, aunque no existe ningún estudio relevante sobre el efecto de muestrear a diferentes resoluciones sobre la estimación de los parámetros de la combustión. 


\subsection{Sistemas de medida de la Presión en cámara}

\subsubsection{Sensores y Acondicionadores}

En apartados anteriores, se mencionaron los diferentes tipos de sensores usados para la medida de la presión en cámara. El uso eficiente de cada tipo de sensor queda condicionado al objetivo para el que se va a registrar la presión. Por ello, se resumirá a continuación los tipos principales de sensores usados para medir la presión en cámara, indicando para cada uno las posibilidades y limitaciones que presenta partiendo de los parámetros estándares que influyen en la elección de un sensor o otro.

\subsubsection{Parámetros para elección de sensores de presión}

El ambiente al que se va a someter el sensor de medida de presión en cilindro es muy exigente. Generalmente, y desde el punto de vista químico, dentro del cilindro se generan productos de la combustión, entre otros, dióxido de carbono, agua, cloruros, hidrocarburos parcialmente quemados, así como óxidos de nitrógeno. Los componentes ácidos son los más perjudiciales para los materiales en contacto con los gases de combustión. Dichos componentes, son más peligrosos cuando existen mayores impurezas en el combustible, principalmente impurezas de azufre que dan lugar a ácido sulfúrico de alto poder corrosivo [38].

Desde el punto de vista de solicitaciones mecánicas y térmicas, se puede alcanzar respectivamente presiones de 300 bares en motores diésel sobre alimentados y temperaturas de los gases por encima de los $1700^{\circ} \mathrm{C}$ de pico.

Así, cualquier tipo de sensor de presión para motores diésel, está sometido a condiciones química (corrosión) y físicas (vibraciones, temperaturas altas, presiones altas, choques térmicos, etc.) muy exigentes. Esas condiciones deben ser cuidadosamente tenidas en cuenta a la hora de decidir, entre otros, algunos parámetros cruciales que tienen que considerarse a la hora de elegir el sensor óptimo. Estos parámetros son:

Rango de medida: determina el rango de presiones que el sensor es capaz de detectar con precisión manteniendo su seguridad. Una medida fuera de rango puede romper el sensor o dar una lectura errónea debido al cambio de la linealidad del sensor fuera de su rango natural de funcionamiento. Los sensores deben ser capaces de soportar picos de presión que puedan salir del rango en condiciones de funcionamiento especial. Por lo tanto, junto con el rango de medida, debe considerarse además el tiempo en el que el sensor puede estar sometido a presiones fuera de su rango nominal. 
Sensibilidad: determina la variación de la característica eléctrica (Ohmios, Culombio, etc.) frente a una variación del efecto mecánico a cuantificar (presión). A medida que el valor absoluto de la sensibilidad es mayor, la conversión de esa característica eléctrica en voltaje o corriente es más fácil.

Rango de temperaturas: es el intervalo de temperaturas nominales al que el sensor puede estar expuesto.

Linealidad: la linealidad de un transductor es la calidad de mantener una sensibilidad constante en todo el rango de medida. Suele expresarse como desviación típica normalizada respeto al máximo valor de medida permitido (fondo de escala). En caso de no linealidad del sensor, hay que tener bien identificada la no-linealidad, la norepetitividad o fenómenos de histéresis que permitan identificar el comportamiento de la sensibilidad en todo el rango de funcionamiento del sensor.

Dimensiones: tienen mayor importancia en caso de sensores que afectan al volumen donde se va a realizar la medida. El caso de medida de presión en cilindro es un caso especial puesto que unas dimensiones grandes afectan al volumen del cilindro además de la dificultad de montaje que presentan estos sensores.

Frecuencia de resonancia: la respuesta de los sensores al someterles a una fuente de excitación oscilatoria es función de la frecuencia de dicha fuente. La frecuencia de resonancia es aquella que provoca una respuesta del sensor de máxima amplitud. Alrededor de esa frecuencia, el comportamiento dinámico de las señales muestreadas deja de ser plano y los componentes de la señal con unas frecuencias cercanas a la de resonancia se ven amplificadas, lo que distorsiona la señal. Conviene que la frecuencia de resonancia del sensor este suficientemente alejada de las frecuencias contenidas en la señal de presión en cámara.

Respuesta dinámica: determinada por dos factores principales:

- El tiempo de respuesta o constante de tiempo, que refleja el tiempo necesario para que el sensor alcance su valor final de lectura después de ser excitado con un escalón de presión.

- Sobreoscilación, que determina el rizado máximo alcanzado por el sensor antes de estabilizarse su respuesta ante un escalón de presión.

Si se necesita un número elevado de muestras de presión a lo largo de un ciclo termodinámico, la respuesta dinámica del sensor tiene que contar con una constante de tiempo pequeña y una sobreoscilación prácticamente nula. Pero, si la finalidad de la medida es identificar fenómenos puntuales (p .ej. presión máxima) la respuesta dinámica no es un factor demasiado importante. 
Sensibilidad térmica, error por choque térmico y deriva térmica: la sensibilidad térmica expresa el efecto de la temperatura del entorno sobre los elementos constructivos del sensor y, por lo tanto, sobre su comportamiento [39]. Esta sensibilidad debe ser muy baja ya que, de lo contrario, se suelen producir dos tipos de deriva indeseable a lo largo de tiempo de medida:

El primero, llamado deriva térmica rápida (short-term drift), se caracteriza por la aparición de errores de medida a lo largo de un ciclo de combustión. Es causado por gradientes altos de temperatura de los gases de combustión (hasta $2000^{\circ} \mathrm{C}$ en pocos milisegundos), por lo que los materiales del sensor pueden tener una dinámica lenta para recuperar su estado normal. La magnitud de este error por choque térmico depende (además del sensor) de la posición del sensor, del régimen de giro y de la carga del motor.

El segundo tipo, llamado deriva térmica lenta (long-term drift), se caracteriza por una acumulación del error por choque térmico a lo largo de tiempo originado por dos causas: el efecto de la temperatura media sobre el cristal y el efecto del primer tipo de deriva térmica, debido a que al final de cada ciclo de combustión existe un error que se va acumulando con el tiempo. Este tipo de deriva térmica toma mayor importancia cuando hay cambios bruscos en el régimen del motor o en la carga.

Compensación de aceleraciones: los sensores de presión deben estar aislados de vibraciones ajenas al fenómeno a medir. Los fabricantes de sensores usan geometrías, tolerancias y materiales especiales para compensar las aceleraciones de los elementos de medida generadas por consecuencia de dichas vibraciones.

\subsubsection{Sensores de presión en cámara Piezorresistivos}

Como se comentó anteriormente, esos sensores se basan sobre el fenómeno de cambio de la resistencia eléctrica de ciertos materiales cuando se someten a presiones. Generalmente estos sensores se caracterizan por el reducido rango de presiones ( 0 a 10 bares) y de temperaturas (hasta $180^{\circ} \mathrm{C}$ ) de funcionamiento. Eso los hace óptimos para medidas de presiones en admisión y en escape (aunque generalmente necesitan refrigeración). Sin embargo, los fabricantes de este tipo de sensores se han interesado en su uso para medir la presión en cilindro. Para conseguirlo, se suele implementar entre el diafragma (material con superficie directamente en contacto con la atmósfera del cilindro) y la superficie del elemento piezoresistivo del sensor, diversos materiales o espacios que permiten exponer el sensor a condiciones menos exigentes de temperatura y presión (figura (3.2)).

Los sensores piezorresistivos adaptados para la medida de la presión en cámara pueden alcanzar un rango de trabajo hasta 250 bares de presión y en condiciones extremas de $450^{\circ} \mathrm{C}$ de temperatura del sensor. El mayor reto para los fabricantes de este tipo de sensores es disminuir la sensibilidad térmica, compensar las aceleraciones y aumentar su vida útil.

Las ventajas de estos sensores consisten, por una parte en el bajo nivel de ruido que aportan las medidas debido a su mayor tolerancia a vibraciones y por otra parte en el mejor referenciado de la presión puesto que la resistencia eléctrica del sensor es directamente proporcional a la presión medida. 


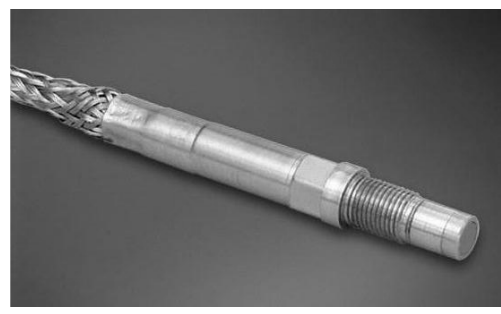

(a) Sensor

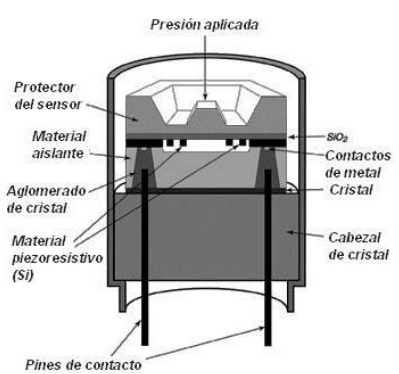

(b) Esquema

Figura 3.2: Sensor de presión en cámara Piezorresistivo Kulite ETMER-CS-190

Los acondicionadores de este tipo de sensores tienen la función principal de fuente de alimentación. La lectura es amplificada y tratada de forma analógica para compensar efectos de temperatura y/o vibraciones.

\subsubsection{Sensores de presión en cámara ópticos}

El elemento común de estos sensores es una membrana deformable por efecto de la presión. La presión a registrar se obtiene mediante la cuantificación de dicha deformación que es proporcional a la presión aplicada.

Los sensores ópticos tradicionales usan fotodiodos para detectar directamente el efecto de la deformación de la membrana, bien calculando el ángulo deformado con un captador óptico compuesto de varios fotodiodos (figura (3.3a)) o bien midiendo la intensidad de luz recibida directamente (figura (3.3b)).

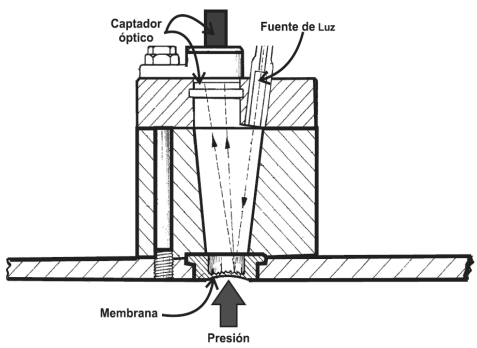

(a) Con múltiples fotodiodos

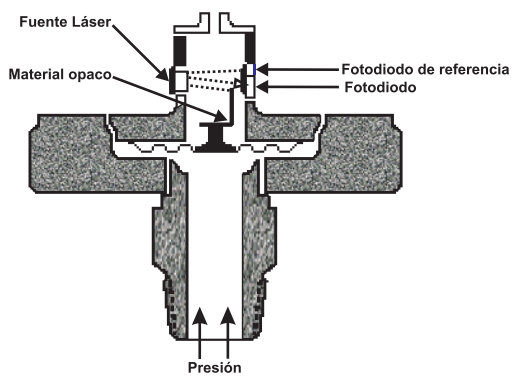

(b) Por intensidad luminosa

Figura 3.3: Sensores ópticos para presión en cámara

Numerosos trabajos muestran la fiabilidad de estos sensores, Wlodarcyk [40]afirma que estos sensores tienen menos de un $2 \%$ de error para presiones altas y baja sensibilidad 


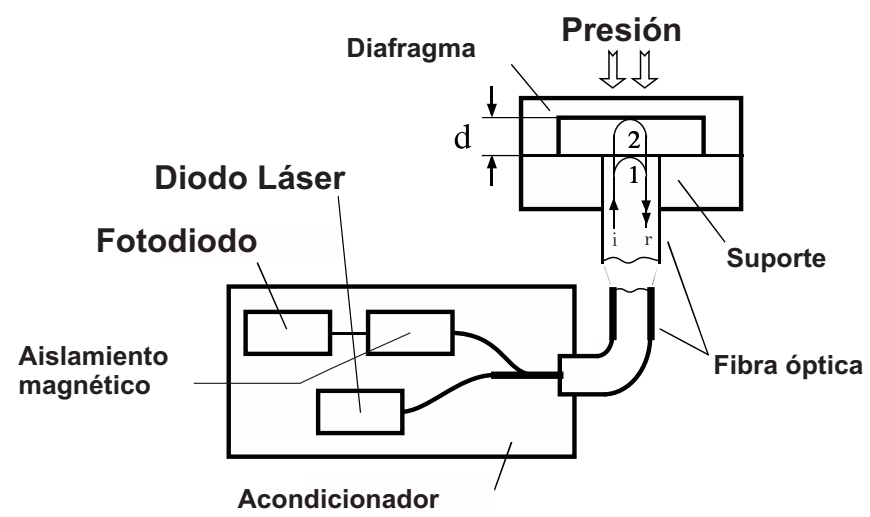

Figura 3.4: Sensores de fibra óptica para presión en cámara

térmica. El mayor problema de estos sensores radica en la calidad de los diafragmas empleados. A largo plazo, dichos diafragmas se dilatan y/o agrietan, lo que distorsiona las medidas. Los fabricantes de este tipo de sensores suelen estimar su vida útil en varios cientos de millones de ciclos.

Los sensores ópticos de presión más avanzados han optado por el uso de la fibra óptica (figura (3.4)). Estos sensores utilizan el desplazamiento de fase de una fuente de luz para medir la presión. La luz se transmite a través de una fibra óptica con componentes directamente en contacto con el diafragma. Al deformarse el diafragma por efecto de la presión, se desplazan determinados componentes especiales de la fibra óptica, lo que lleva a cambiar la sección de paso del haz de luz [41]. La luz que recorre la fibra óptica es medida por un analizador de espectros ópticos. El cambio espectral entre la fuente y la luz recibida es proporcional a la presión aplicada.

Varios estudios reflejan la fiabilidad de estos sensores para medida de la presión en cámara. Algunos como Wlodarczyk y otros [42] aseguran un 1\% de error y muy baja sensibilidad térmica. Otros como Atkins y Wenderker [41-44] muestran varias posibilidades de implementar este tipo de sensores presentando resultados fiables para dichas posibilidades. .

La ventaja de estos sensores es la baja sensibilidad térmica que presentan. Como desventaja principal se encuentra el significante ruido presente en la medida así como la dificultad que presenta implementar complicados algoritmos para el análisis espectral necesario o, en su caso, la utilización de otros acondicionadores de señal (filtros o interferómetro del mach-Zehnder) para traducir las magnitudes eléctricas medidas por este tipo de sensores. 


\subsubsection{Sensores de presión en cámara piezoeléctricos}

Se basan en el fenómeno piezoeléctrico que consiste en la aparición de cargas eléctricas al aplicar una fuerza o aceleración exterior sobre materiales como el cuarzo (SiO2), titanio de bario (BaTiO3), Orto-fosfato de Galio(GaPO4) u otros materiales sintéticos $[20,45]$. Este fenómeno proporciona una tecnología de medida en condiciones extremas de temperatura, de corrosión o de altas presiones con dinámica de comportamiento rápida.

Los efectos piezoeléctricos fueron descritos por primera vez por los hermanos Pierre y Jacques Curie en 1880. "Piezein" proviene del griego y significa comprimir o aplicar una presión. Los dos físicos descubrieron que las superficies de algunos cristales están cargadas eléctricamente cuando el cristal se somete a una tensión mecánica. Esta carga eléctrica es directamente proporcional a la fuerza ejercida sobre el cristal y se mide en pico-coulomb (pC).

Sin embargo, estos efectos no pasaron de ser más que una curiosidad científica durante más de 30 años. La primera publicación sobre la medición de la presión con sensores piezoeléctricos en los motores de combustión interna apareció en 1925 [46]. A finales de la década de 1940 se comenzó el desarrollo y fabricación de sensores de presión piezoeléctricos a mayor escala para su utilización en el desarrollo de motores.

Junto con las patentes de amplificador de carga de Walter P. Kistler en 1950, la tecnología de medición con transductores piezoeléctricos abrió la puerta para la medición dinámica de las variables mecánicas con gran precisión. En 1958, se puso en marcha el primer sensor de presión en miniatura de aplicación universal que llegó a establecer un nuevo estándar en la medición de la presión. En las décadas de los años 60, 70 y 80 los fabricantes siguieron perfeccionando los materiales piezoeléctricos que utilizan los sensores, buscando nuevos cristales y diversas formas para su mecanización. Se consiguió en esas décadas elevar las prestaciones de dichos sensores y sus tamaños permitiendo mayor facilidad para su instalación en las cámaras de motores.

El definitivo establecimiento del uso de los sensores piezoeléctricos se produjo con la aparición del amplificador de carga en 1950, aparato imprescindible que acondiciona la señal fuente del sensor convirtiéndola de una señal proporcional a la carga eléctrica del cristal piezoeléctrico a un voltaje proporcional a la presión medida. Así, en las décadas de los años 80 y 90 hubo mayor interés por desarrollar mejores amplificadores de carga que de introducir nuevos materiales en la fabricación de los sensores piezoeléctricos. Interés justificado por el rápido avance de la electrónica en esas décadas.

Desde el inicio del siglo XXI y hasta nuestros días, y bajo la demanda de la mayoría de los fabricantes de motores, los fabricantes de sensores piezoeléctricos muestran un mayor esfuerzo para lanzar al mercado sensores piezoeléctricos de bajo coste para que puedan ser viables económicamente en su uso para automoción y dejen de estar restringidos a la investigación. Hoy en día ya existen vehículos comerciales con sensores de presión en cámara instalados: Toyota a finales de los 80, Audi (Q7) y Honda (iDTEC) en el 2008, GM (Cadillac-CTS) en el 2009 y Opel (Vectra).

Los captadores piezoeléctricos utilizados actualmente en la investigación, se caracterizan por su robustez y sensibilidad. Aunque presenten problemas de estabilidad 

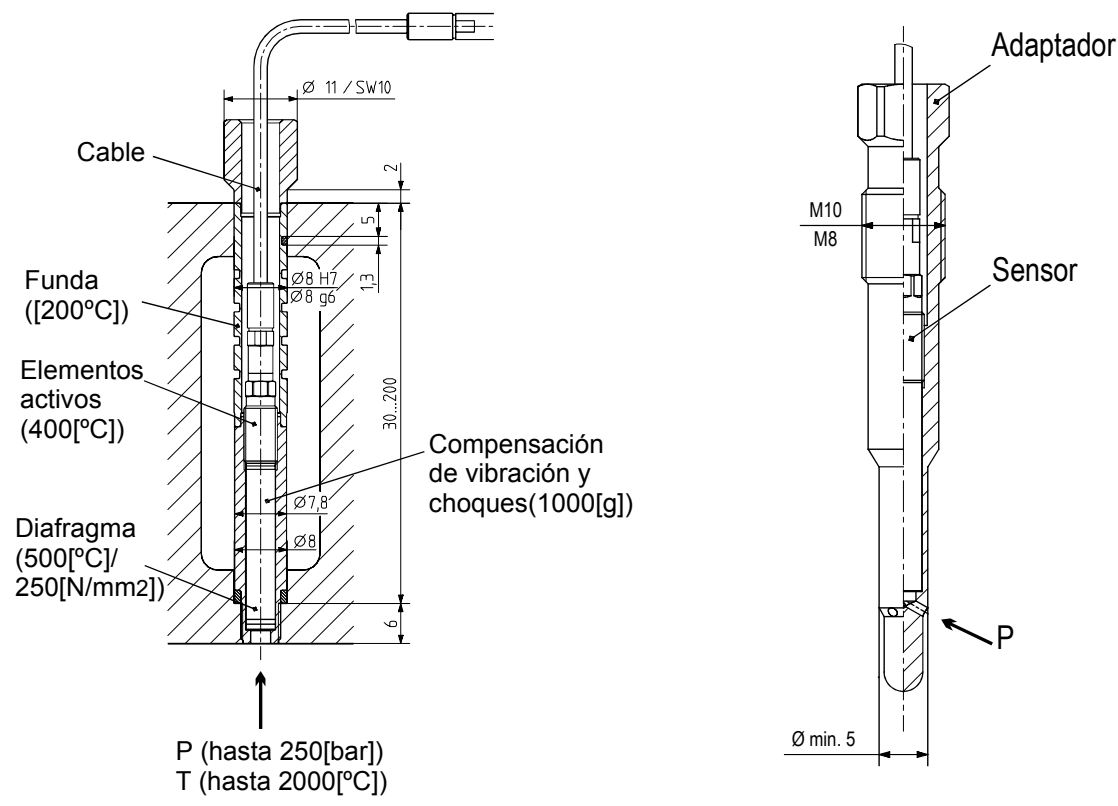

Figura 3.5: Sensor piezoeléctrico y adaptador

a largo plazo, cierta influencia a los efectos de la temperatura y aceleración, así como posibilidad de aparición de parásitos eléctricos, siguen siendo prácticamente insustituibles para la medición de la presión en cámara [17].

El cristal de cuarzo, elemento principal de estos sensores, presenta una vida útil infinita, pues la estructura molecular del cristal no puede modificarse mientras no se excedan los rangos de funcionamiento. Sin embargo, el cuerpo del sensor puede dañarse con el tiempo y perjudicar las medidas. Elementos como el diafragma que permite transmitir al cristal la variación de la presión o compensadores de aceleraciones suelen deteriorarse después de varios años de funcionamiento.

Al ser estos sensores intrusivos (el sensor tiene que estar en contacto con los gases encerados en el cilindro) se debe tener en cuenta la ubicación del sensor [47]. Los fabricantes aportan varios diseños ya sea para montar estos sensores por mecanizado de la culata, como para introducirlos en el cuerpo de otros elementos como la bujía de calentamiento para motores diésel (figura (3.5)). Además, en algunos sensores, se suelen implementar circuitos de refrigeración con los que se consigue atenuar el efecto de la sensibilidad térmica. Esta solución demanda mayor tamaño del sensor, lo que no es siempre posible. Sin embargo los sensores no refrigerados aportan precisiones elevadas, ya que incorporan diafragmas capaces de proteger el cristal de las altas temperaturas de los gases, además, un montaje correcto hace que el flujo de calor se disipe por la culata [48].

Los captadores piezoeléctricos son, con diferencia, los más empleados para medir la 
presión el cilindro $[12,35,49,50]$ y los que se van a considerar en este trabajo. De hecho, estos sensores son usados como referencia para estudiar las prestaciones de cualquier otro tipo de sensores [40]. Su sensibilidad alcanza -90pC/bar, lo que les permite una precisión elevada.

\subsubsection{Características de la señal}

Partiendo de que se van a emplear sensores piezoeléctricos para la medida de la presión en cámara, y para definir los requisitos del sistema de medida, conviene recurrir a la experiencia para determinar las características básicas de la señal a medir y las diferentes etapas transcurridas desde la captación de la variación de la presión hasta su registro.

Como se comentó anteriormente, los cristales piezoeléctricos cambian de carga $(q)$ al estar sometidos a una fuerza exterior, de manera que estos cristales aportan una variación de carga $\left(\frac{d q}{d t}\right)$ proporcional a la variación de presión $\left(\frac{d p}{d t}\right)$. Desde el punto eléctrico, un cristal piezoeléctrico corresponde a un circuito equivalente simplificado de Butterworth-Van Dyke [51], como el mostrado en la figura (3.6a), donde los componentes eléctricos son equivalentes a las propiedades mecánicas del cristal:

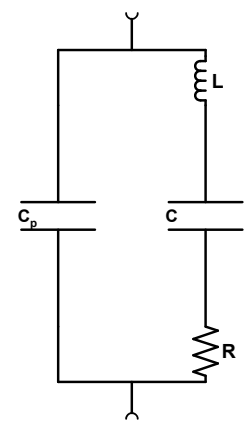

(a) Circuito equivalente del cristal piezoeléctrico

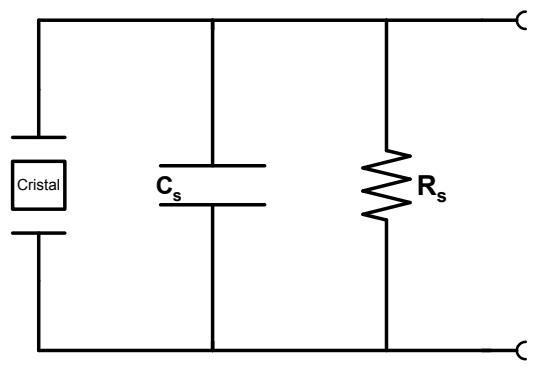

(b) Circuito equivalente del sensor de presión piezoeléctrico

Figura 3.6: Circuitos equivalentes

- La rama izquierda de este circuito: llamada la rama estática y sólo contiene las capacitancia que representa la capacidad del cuarzo $(C)$ y las capacidad de las conexión externa $\left(C_{p}\right)$.

- La rama derecha se conoce como la rama mocional y representa las resonancias acústicas en el cuarzo y su carga. $R$ representa las pérdidas debidas a la fricción mecánica, $L$ es equivalente a la masa del cristal en vibración y $C_{s}$ indica el relajamiento mecánico del cristal. 
El circuito equivalente presentado en la figura (3.6b) representa el cristal y las conexiones internas del sensor, siendo este circuito el usado para analizar las propiedades de los sensores de presión piezoeléctricos. De hecho, el análisis de las magnitudes del diagrama de Bode de este circuito (figura (3.7)) aporta información sobre el rango óptimo de frecuencias del sensor tomando como criterio una distorsión en la amplitud inferior al $5 \%$ ( equivalente a $3 \mathrm{~dB}$ ). Este rango viene limitado por una frecuencia de corte inferior determinada por la constante del tiempo $\tau=R_{s} . C_{s}$ y que produce un efecto de deriva en la señal. Y por $0,2 \cdot f_{n}$ siendo $f_{n}$ la frecuencia natural del cristal.

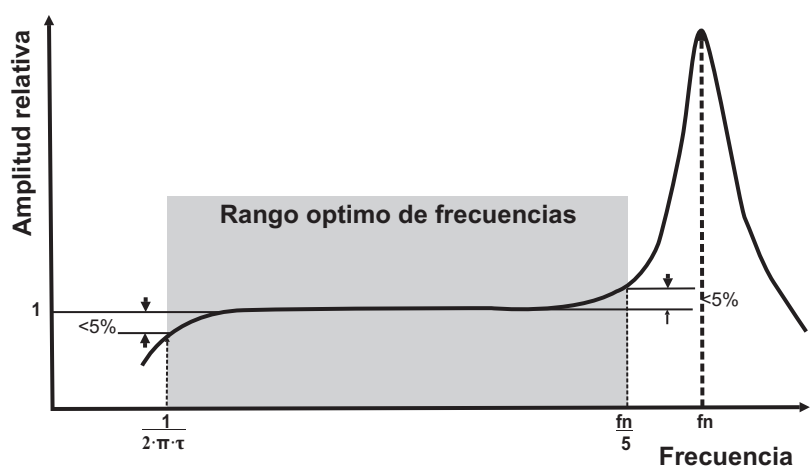

Figura 3.7: Representación típica del diagrama de Bode de magnitudes para sensores de presión piezoeléctricos

A nivel práctico, un valor típico de $f_{n}$ ronda los $150 \mathrm{kHz}$. Cualquier componente de presión en cámara con frecuencia superior a $30 \mathrm{kHz}$ se ve amplificada, pudiendo dar lugar al fenómeno de resonancia del cristal y falsificando así las medidas. Ante frecuencias muy bajas, el comportamiento se ve afectado por las cargas térmicas aplicadas al sensor (deriva térmica) y por las impedancias de carga que se conectan al sensor, es decir, las impedancias del cable que transmite la carga y del acondicionador de carga.

Los cables que transmiten la carga deben tener una alta resistencia térmica y química, ya que la conexión eléctrica entre el sensor piezoeléctrico y el acondicionador de carga debe estar muy aislada, aproximadamente $R_{c}=10^{13} \Omega$. El ruido proporcionado por la excitación de las constantes dieléctricas del cable se suma al ruido que puede aparecer por vibración del cable, lo cual produce pequeñas cargas en el conductor (efecto triboeléctrico) que son equivalentes a un condensador de capacitancia $C_{c} \backsim 1 p C$. Los fabricantes evitan este ruido implementando capas intermedias especiales recubiertas con grafito en los cables. Aun así, la capacitancia de los cables se ve atenuada en función de la longitud de los mismos. Un cable específico de $2 \mathrm{~m}$ para transmitir la carga suele tener un rango de frecuencias efectivas, o un ancho de banda, aproximadamente de $180 \mathrm{kHz}$. Este ancho se ve reducido a $130 \mathrm{kHz}$ para cables de $20 \mathrm{~m}$ de longitud. 
Una variación de carga eléctrica del orden de varios picoculombios, se traduce en una corriente eléctrica $(i)$ tal como se presenta en la ecuación 3.3.1:

$$
i=\frac{d q}{d t}=k \cdot \frac{d p}{d t}
$$

donde $(k[p C / b a r])$ presenta la constante de proporcionalidad que se aproxima a la sensibilidad del sensor.

Para obtener la magnitud de la presión, se recurre a la integración de esta ecuación:

$$
p_{t_{1}}-p_{t_{0}}=\frac{1}{k} \cdot \int_{t_{0}}^{t_{1}} d q=\frac{1}{k} \cdot \int_{t_{0}}^{t_{1}} i \cdot d t
$$

Esta integración se materializa mediante amplificadores de carga que convierten la carga en voltaje proporcional a la presión aplicada al sensor piezoeléctrico. La etapa de entrada de un amplificador de carga cuenta con un circuito de realimentación capacitiva que equilibra el efecto de la señal de carga de entrada aplicada. La señal de retorno representa, por tanto, el valor de la carga de entrada.

La figura(3.8) representa el circuito equivalente al conjunto sensor-cable y amplificador.

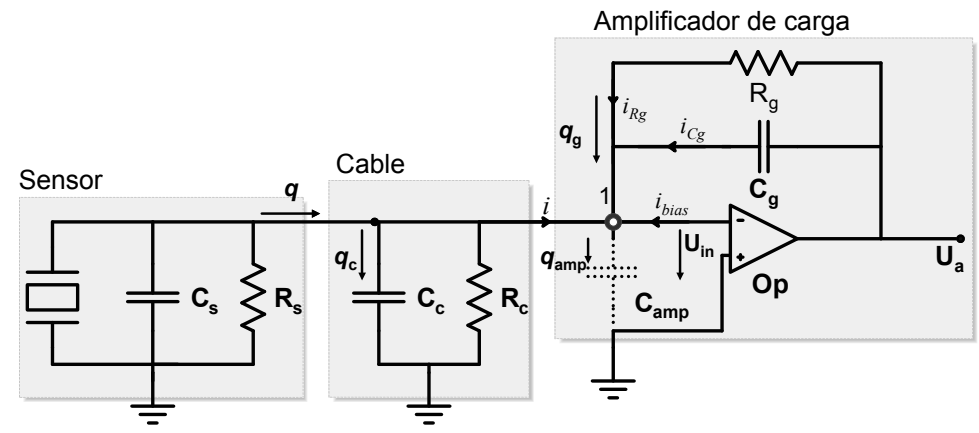

Figura 3.8: Circuito equivalente del conjunto sensor-cable-amplificador de carga

Si se desprecian las pérdidas de carga en las resistencias, la carga $q$, generada por el sensor, se aplica a la entrada inversora del amplificador operacional y se distribuye entre el condensador $C_{c}$ del cable, el condensador $C_{a m p}$ que representa la capacidad del amplificador operacional y el condensador $C_{g}$ de retroalimentación de la etapa principal del amplificador de carga:

$$
q=q_{c}+q_{a m p}+q_{g}
$$

Aplicando la ecuación electrostática que relaciona la carga con el voltaje $(q=C \cdot U)$ se obtiene:

$$
q=U_{a m p} \cdot\left(C_{c}+C_{a m p}\right)+U_{g} \cdot C_{g}
$$


Considerando que se trata de un amplificador operacional ideal, la tensión en la entrada inversora debe ser igual a la tensión de la entrada no-inversora, $U_{i n}=U_{a m p}=0$. Y por lo tanto:

$$
q \simeq U_{g} \cdot C_{g}=U_{a} \cdot C_{g} \Longleftrightarrow U_{a}=\frac{q}{C_{g}}
$$

El resultado muestra claramente como, en el caso ideal, la tensión de salida de un amplificador de carga depende sólo de la carga de entrada y la capacidad de retroalimentación $C_{g}$, que debe ser seleccionada en función del rango de medidas. Las capacitancias de entrada y de cable no tienen ninguna influencia sobre la tensión de salida. Esta es la ventaja principal de los amplificadores de carga.

Como se demostrará a continuación, la resistencia de retroalimentación $R_{g}$ tiene la función de proporcionar estabilidad del nivel de continua en el circuito y de definir el límite inferior de frecuencia del amplificador. Dicha resistencia debe ser lo bastante elevada para eliminar las cargas residuales del condensador $C_{g}$ en un tiempo óptimo.

De la aplicación de la ley de Kirchoff al nodo 1 de la figura (3.8) se obtiene:

$$
i+i_{R_{g}}+i_{C_{g}}+i_{\text {bias }}=0
$$

donde $i_{\text {bias }}$ representa las corrientes de polarización ("Bias") de entrada del amplificador operacional que al considerarlo ideal son nulas.

Teniendo en cuenta la ecuación 3.3.1:

$$
\frac{d q}{d t}+C_{g} \cdot \frac{d U_{a}}{d t}+\frac{U_{a}}{R_{g}}=0
$$

Resolviendo esta ecuación diferencial, se obtiene la respuesta temporal que relaciona la carga con el voltaje de salida del amplificador de carga:

$$
U_{a}=\frac{-q}{C_{g}} \cdot e^{-t / R g \cdot C g}
$$

La figura (3.9) representa la respuesta temporal de un amplificador de carga en función de distintas resistencias $R_{g}$, así como la respuesta a bajas frecuencias.

La respuesta a bajas frecuencias se traduce en efecto de deriva (Drift [46]) sobre la medida. Las cargas residuales se acumulan a lo largo del tiempo saturando en ocasiones el amplificador de carga. Para evitar este efecto, la resistencia $R_{g}$ debe ser cuidadosamente seleccionada. Un valor muy elevado $\left(R_{g} \simeq 10^{14} \Omega\right)$ de la resistencia no elimina las cargas residuales, sin embargo, es óptimo para medida de señales cuasi-estáticas o para la calibración. Valores pequeños de la resistencia $\left(R_{g} \simeq 10^{9} \Omega\right)$ permiten descargar rápidamente la carga (actuando como filtros de paso alto), siendo óptimos para medidas donde interese la dinámica de la señal pero presentan mayor error. 

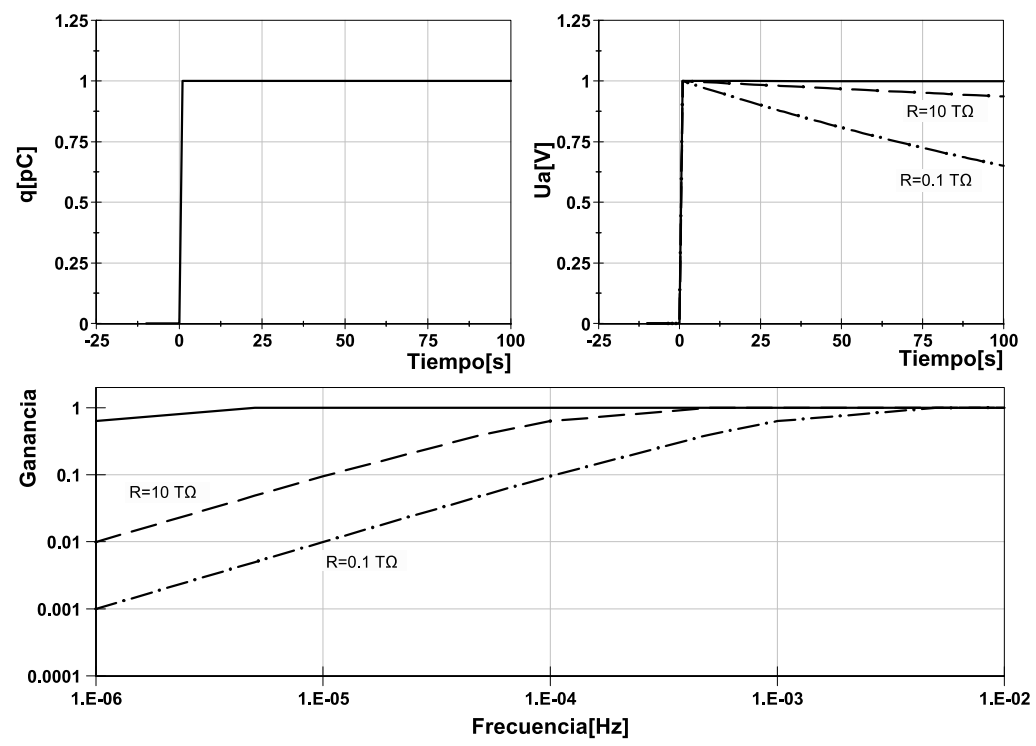

(a) Respuesta típica temporal y en frecuencia de un amplificador de carga.
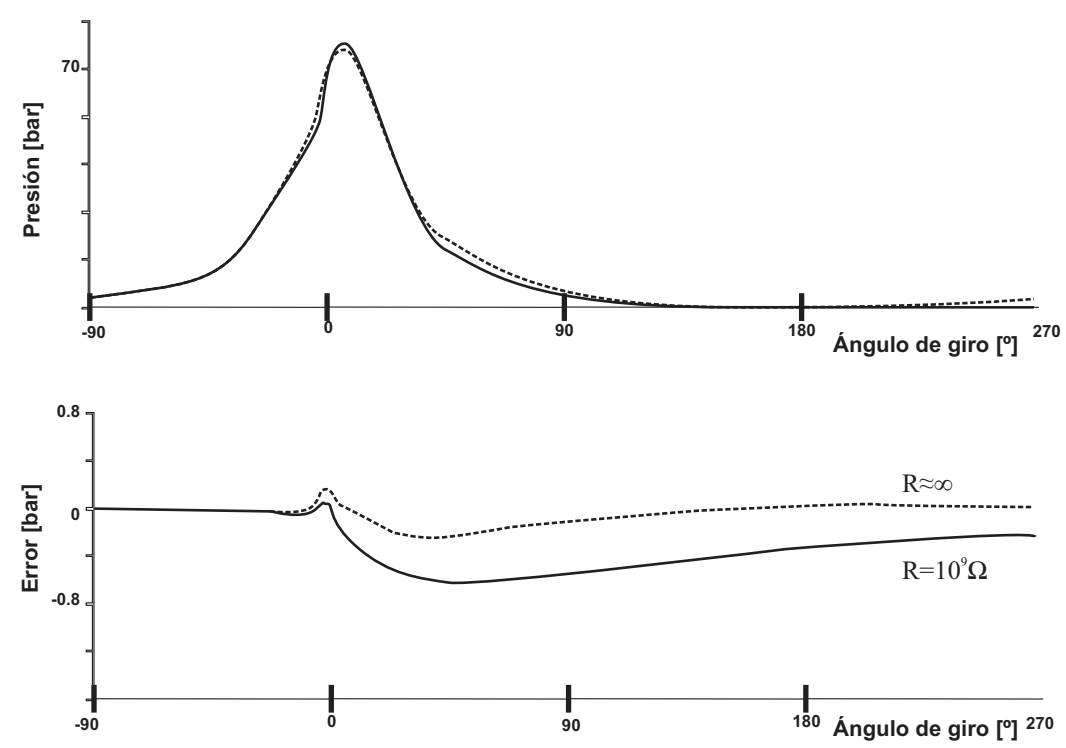

(b) Efecto de deriva en la señal de presión a bajo régimen de giro (sensor con poca sensibilidad).

Figura 3.9: Respuesta típica temporal y en frecuencia de un amplificador de carga. 
En la figura (3.9b) se aprecia que para incrementos significativos de la presión en cámara (durante la compresión), el error es menor al usar amplificadores de carga con $R_{g}$ pequeña. En cualquier caso, durante la compresión, el error cometido con distintas magnitudes de $R_{g}$ es prácticamente despreciable. Sin embargo, en la fase de escape el error es mayor, sobre todo para resistencias pequeñas, pues aunque la presión en cámara cambia de forma suave, la descarga rápida del condensador $C_{g}$ hace que la tensión de salida del amplificador de carga disminuya de forma más brusca, lo que hace aumentar el error.

Para eliminar la deriva debida a las cargas residuales, se puede forzar la descarga del condensador $C_{g}$ cortocircuitándolo mediante un circuito de compensación de deriva que tendrá que actuar cuando la carga en el sensor sea nula. Para la medida de presión en cámara, sería óptimo usar resistencia muy grande $\left(R_{g} \simeq 10^{11} \Omega\right)$ y cortocircuitar al final de cada ciclo de motor. De esta forma, se puede eliminar también los errores debidos a la deriva térmica acumulados a lo largo de la medida (long-term drift).

El conjunto sensor - cable - amplificador de carga tiene un comportamiento estable en la zona óptima de frecuencias $\left[\frac{1}{2 \cdot \pi \cdot R_{c} \cdot C_{c}}, 30 \mathrm{kHz}\right]$ (figura (3.7)). Sin embargo, esta zona se ve afectada por fenómenos debidos al viaje de la onda de presión en la cavidad impuesta por el adaptador o por el propio mecanizado para introducir el sensor (figura (3.5)). En dicha cavidad se producen ondas con frecuencias de resonancia (ecuación (3.3.4)) mucho más bajas que la frecuencia natural del sensor. En la práctica esta frecuencia ronda los $18 \mathrm{kHz}$.

$$
f_{\text {cav }}=\frac{\text { velocidad del sonido }}{4 \cdot \text { Longituddelacavidad }}
$$

Este fenómeno, conocido como oscilaciones en el tubo (pipe oscillations), tiene una frecuencia $\left(f_{\text {cav }}\right)$ que depende de la geometría de la cavidad y de la velocidad del sonido (ecuación (3.3.4)). A medida que aumenta la longitud de la cavidad, las oscilaciones disminuyen significativamente, sin embargo, se transmiten mayores cargas térmicas provocando sobrecalentamiento y por lo tanto la alteración en la sensibilidad térmica del sensor $[39,48]$. Dado que la velocidad del sonido depende del cociente de calores específicos, de la constante específica de los gases y principalmente de la temperatura, y aumentando ésta cuando inicia la combustión, se producen oscilaciones en la medida de presión en cámara, debidas justamente a este aumento de temperatura.

La tensión de salida del amplificador de carga suele ser acondicionada para eliminar ruidos antes de su amplificación. Generalmente, se usan filtros de paso alto (para eliminar ruidos de baja frecuencia y parte de los efectos de deriva) y filtros de paso bajo (para eliminar ruidos eléctricos de alta frecuencia). El diseño de esta etapa es de suma importancia, debido a que, cualquier filtro impone un desfase en la señal y una pérdida de información existente en la misma.

Por este motivo, hay que elegir cuidadosamente las frecuencias de corte y las características de los filtros. El punto de partida de diseño de los filtros es la elimincaión de las frecuencias altas debidas a las resonancias, tanto la del sensor como las debidas a la cavidad "pipe oscillations". Estos filtros eliminan el efecto del "aliasing" que surge en el muestreo, deben ser electrónicos (así el filtrado es continuo y no discreto) y tener una 
frecuencia de corte máxima equivalente a una quinta parte la frecuencia natural del cristal (figura (3.7)). En el capítulo 5 se desarrollara más el diseño de los filtros como parte del tratamiento de la señal de presión en cámara.

En resumen, la conversión de la señal de la presión en cámara a voltaje se ve alterada por cuatro factores principales:

- Efecto de la deriva debida a la respuesta dinámica del conjunto sensor, cable y amplificador de cargas. Este efecto se puede atenuar eligiendo adecuadamente los parámetros $R_{g}$ y $C_{g}$ del amplificador de carga.

- Efecto de deriva debida a las cargas térmicas sobre el sensor. Este efecto se atenúa eligiendo sensores con sensibilidad térmica baja o sensores refrigerados.

- Efecto de las oscilaciones que aparecen como consecuencia de la circulación de la onda de presión en la cavidad debida al montaje del sensor. Dichas oscilaciones se pueden eliminar mediante un filtrado apropiado si no se puede evitar la cavidad.

- Efecto de acondicionamiento y filtrado de la señal. Se traduce en un desfase y/o una distorsión de la señal.

A estos factores, se les puede sumar el efecto de la linealidad del sensor, efecto que se evalúa mediante el proceso de la calibración. En el presente trabajo se ha utilizado la calibración estática basada en aplicar, con equipos especiales, una presión conocida y medir el voltaje de salida del amplificador de carga. La viabilidad de este proceso está justificada en varios trabajos $[2,10,35,52,53]$.

Para caracterizar el efecto de la linealidad de toda la cadena del sistema de medida, se realiza la calibración de todo el conjunto (sensor, cable, amplificador de carga y sistema de medida).

\subsubsection{Criterios de selección del sistema de medida}

El sistema de medida convierte una señal analógica (voltios) en un número (binario) mediante convertidores analógicos / digitales (CA/D "Analog-to-Digital Converter"). La calidad de los datos generados por el sistema de medida se evalúa haciendo uso de los siguientes parámetros:

- Relación señal-ruido $(S N R)$ de cuantización.

- Resolución.

- Frecuencia de muestreo o Resolución temporal. 


\subsubsection{Resolución.}

La señal analógica, que varía de forma continua en el tiempo, se somete a un muestreo a una velocidad fija (frecuencia de muestreo), obteniéndose así una señal digital compuesta por una serie de valores. Cada valor se expresa con un número de bits equivalente a la resolución $(\Delta)$ del convertidor A/D.

El error cometido al convertir la señal analógica en un valor digital, llamado error de cuantificación, depende de la linealidad del convertidor, la temperatura y ambiente de trabajo y, principalmente, de la resolución del convertidor. Este error suele ser cuantificable por la mitad del rango de la señal analógica que corresponde a la variación del bit menos significativo (LSB o "Least Significant Bit") del valor digital, tal y como indica la ecuación (3.3.5), donde $B$ presenta la longitud en bits de la muestra (resolución digital) y $F S$ el rango de medida.

$$
\mid \text { Errordecuantificación }[V] \mid \leq \frac{\Delta}{2}=\frac{F S[V]}{2^{B}}
$$

En general [54], el error de cuantificación provoca un ruido blanco uniforme en la medida. Su efecto se evalúa mediante la relación señal - ruido de cuantización ( $S N R_{Q}$ “Signal-to-Quantization-Noise Ratio"), que se define como la relación, en decibelios, entre la potencia de la señal y la potencia de la señal originada por el error de cuantificación.

$$
S N R_{Q}[d B]=10 \cdot \log \frac{P_{\text {señal }}}{P_{\text {ruido }}}=10 \cdot \log \frac{\int_{-\infty}^{+\infty} s^{2}(t) \cdot d t}{\int_{-\infty}^{+\infty} r^{2}(t) \cdot d t}
$$

Si el ruido de cuantificación $(r(t))$ se mantiene uniforme en el rango de amplitudes $\left(\frac{-\Delta}{2}, \frac{\Delta}{2}\right)$, el valor medio del error es, por lo tanto, nulo y la potencia del ruido, con relación al escalón de cuantificación, es la varianza $\left(\sigma^{2}=\frac{1}{\Delta} \int_{\frac{-\Delta}{2}}^{\frac{\Delta}{2}} r^{2} \cdot d r\right)$ de esta distribución uniforme, siempre que el muestreo se haga con la frecuencia de Nyquist.

Es decir:

$$
S N R_{Q}[d B]=10 \cdot \log \frac{P_{\text {señal }}}{\sigma_{r}^{2}}=10 \cdot \log P_{\text {señal }}-10 \cdot \log \frac{\Delta^{2}}{12}
$$

De donde:

$$
S N R_{Q}[d B]=10 \cdot \log P_{\text {señal }}-10,79-20 \log F S+6,02 \cdot B
$$

Al desconocer la potencia de la señal, la determinación de la relación señal-ruido de cuantificación máxima teórica de un convertidor A/D se suele hacer considerando la señal como una señal sinusoidal de amplitud máxima correspondiente al fondo de escala. Eso da lugar a la aproximación presentada en la ecuación siguiente:

$$
\operatorname{SNR}_{Q}[d B]=6,02 \cdot B+1,76
$$


Las ecuaciones 3.3.7 y 3.3.8 sugieren que por cada bit, la relación señal a ruido de cuantización mejora en aproximadamente $6 \mathrm{~dB}$. Un método para seleccionar la resolución del convertidor A/D es fijar el valor de $S N R_{Q}$.

Por otra parte, la elección de la resolución depende del error nominal de la medida, es decir, el error más probable permitido en la medida y que es calculado por la ecuación (3.3.9) [55]:

$$
\text { error }_{\text {nominal }}=\sqrt{\sum \text { errores }^{2}}
$$

Para la medida de la presión en cámara, el rangodemedida $(V)$ corresponde a un rangode medida (bar) o fondo de escala $(F S)$. La tabla (3.2) presenta la relación entre la resolución del convertidor A/D y la resolución en magnitud eléctrica y física obtenida para un rango de voltaje [0..10V] equivalente a un fondo de escala [0..250bar].

\begin{tabular}{|c|c|c|c|c|c|}
\hline $\begin{array}{l}\text { Resolución } \\
\text { [bit] }\end{array}$ & $\begin{array}{l}\text { Resolución } \\
{[\mathrm{mV}]}\end{array}$ & $\begin{array}{l}\text { Resolución } \\
\text { [mbar] }\end{array}$ & $\begin{array}{c}\text { Error } \\
\text { máximo } \\
{[\%]}\end{array}$ & $\begin{array}{c}\text { Peso del } \\
\text { error }^{a}[\%]\end{array}$ & $\begin{array}{l}\text { Peso del } \\
\text { error }^{b}[\%]\end{array}$ \\
\hline 8 & 39.0 & 976.6 & 0.391 & 18.13 & 48.39 \\
\hline 10 & 9.8 & 244.1 & 0.098 & 4.61 & 13.73 \\
\hline 12 & 2.4 & 61.0 & 0.024 & 1.13 & 3.39 \\
\hline 14 & 0.6 & 15.3 & 0.006 & 0.28 & 0.85 \\
\hline 16 & 0.2 & 3.8 & 0.002 & 0.09 & 0.28 \\
\hline
\end{tabular}

Tabla 3.2: Resolución de la medida en función de la resolución del convertidor A/D.

${ }^{a}$ Caso más desfavorable

${ }^{b}$ Caso favorable

Si se trabaja en un rango de presiones hasta 250 bares (suficiente para motores alternativos de automoción actuales), se considera un sensor de baja linealidad ( $0.5 \%)$ y una sensibilidad térmica alta ( $2 \%$ en el caso más desfavorable), además de un conjunto (cable, amplificador de carga) con error típico de $0.5 \%$, se tiene que:

$$
\operatorname{error}_{\text {nominal }}[\%]=\sqrt{0,5^{2}+2^{2}+0,5^{2}+\operatorname{error}_{C A D}^{2}}=\sqrt{4,5+\text { error }_{C A D}^{2}}
$$

Con los valores de error de la tabla (3.2), para el caso más desfavorable, una resolución mayor de 12 bits tiene un peso insignificante sobre el error de la medida (inferior al $1 \%$ ). Incluso para el caso más favorable, donde se considera que el sensor no tiene sensibilidad térmica, una resolución de 12 bits es adecuada.

Una resolución elevada, no asegura mayor exactitud en la medida, solo asegura mayor precisión. A medida que aumenta la resolución el coste económico del sistema de medida y el coste computacional para análisis de datos es más importante.

Lo anterior justifica por que en el presente trabajo, y para temas de investigación, se opta por el uso de sistemas de medida con resolución superior o igual 12 bits. 
Para el trabajo realizado en esta tesis, se ha seleccionando definitivamente, por disponibilidad, un sistema de medida de 16 bits de resolución.

\subsubsection{Frecuencia de muestreo.}

El tiempo que tarda un convertidor analógico/digital en llevar a cabo una conversión es inversamente proporcional a la frecuencia de muestreo expresada en [Hz]. A medida que el convertidor A/D tarde más en proporcionar un valor digital, la frecuencia de muestreo disminuye.

La frecuencia de muestreo $\left(f_{m}\right)$ mínima requerida viene determinada, de acuerdo con el teorema de muestreo de Nyquist-Shannon, por el doble de la frecuencia límite que se desea registrar en la señal de interés. Por lo tanto, sólo se podrán registrar frecuencias por debajo de la mitad de la frecuencia de muestreo, lo que exige que la señal analógica esté limitada en banda (filtrado analógico).

Si no se cumple el teorema de Nyquist y se efectúa el registro con una frecuencia menor que la establecida por el teorema, además de perder información, se produce el efecto de solapamiento ("aliasing") de espectros.

Sin embargo, una frecuencia de muestreo muy elevada (sobre-muestreo) no asegura mejores medidas. De hecho la relación $S N R_{Q}$ de la ecuación (3.3.7) y su aproximación en la ecuación (3.3.8), contemplan un ruido que se extiende por toda la banda de Nyquist. Si se modifica esta banda se deberá añadir una constante para la banda restante. Dicho de otro modo, la aproximación de la ecuación (3.3.8) se reescribe de la forma siguiente:

$$
\operatorname{SNR}_{Q}[d B]=6,02 \cdot\left(B+10 \cdot \log \left(\frac{f_{m}}{f_{\text {Nyquist }}}\right)\right)+1,76
$$

Donde, se aprecia que si se cumple el teorema de Nyquist $\left(f_{m}=f_{N y q u i s t}\right)$, la ecuación (3.3.10) es equivalente a la ecuación (3.3.8).

La ecuación (3.3.10) sugiere que una frecuencia de muestreo 10 veces mayor que la frecuencia de Nyquist, produce el mismo efecto sobre la relación $S N R_{Q}$ que aumentar la resolución en 4 bits (lo que apoya la elección final de una resolución de 16 bits 3.3.3.1).

Para la medida de la presión en cámara, la frecuencia de muestreo tiene que ser capaz de detectar la frecuencia de los fenómenos que se pretenden estudiar. Esto es, la frecuencia de muestreo de la presión en cámara depende de su uso (3.2). Payri [52] y otros confirman que para frecuencias bajas $(<200 \mathrm{~Hz})$ el contenido de la presión en cámara está dominado por la señal de arrastre, mientras que las frecuencias medias $(200 \mathrm{~Hz}$ a 6 $\mathrm{kHz}$ ) son absolutamente dominadas por la señal de la combustión y las altas frecuencias $(6 \mathrm{kHz}$ a $20 \mathrm{kHz}[13,14])$ son una consecuencia de las resonancias relacionadas con la combustión.

Por lo tanto, cuando se usa la presión en cámara para estudio de la combustión, la frecuencia de muestreo del sistema de medida debe ser como mínimo de $12 \mathrm{kHz}$. Además, para evitar el efecto de aliasing, es obligatorio implementar filtros analógicos anti-aliasing con una frecuencia de corte aproximadamente de $6 \mathrm{kHz}$. Esta obligación esta justificada por la imposibilidad de eliminar el aliasing por filtrado digital, pues una vez registrada la señal no se puede asegurar que un componente de baja frecuencia de la señal sea debido al aliasing o no [54]. 
Los filtros anti-aliasing eliminan componentes armónicos por encima de la máxima frecuencia de interés. Más que eliminar, realmente se trata de hacer que los niveles de estos armónicos se reduzcan al umbral de incertidumbre de la señal de entrada. Además, hay que tener muy en cuenta el desfase creado por estos filtros.

Al menos que se registre simultáneamente el ángulo de giro del motor, los sistemas de medida no son capaces de asociar los valores de presión registrados con su correspondiente ángulo de giro. Se precisa entonces una referencia angular para cualquier empleo de la presión en cámara. El efecto de los errores en dicho referenciado esta ampliamente estudiado ( $[2,10,56])$.

En general, se suele utilizar la señal del ángulo de giro, en forma de pulsos, para lanzar el proceso de muestreo. En este caso, la frecuencia de muestreo viene determinada por el régimen de giro instantáneo y la resolución angular equivalente al número de pulsos por revolución como indica la ecuación (3.3.11), donde rpm es el régimen instantáneo de giro en revoluciones por minuto, $N_{\text {pulsos }}$ el número de pulsos por revolución y $\Delta_{\text {angular }}$ es la resolución angular empleada:

$$
f_{m}[H z]=\frac{r p m \cdot N_{\text {pulsos }}}{60}=\frac{6 \cdot r p m}{\triangle_{\text {angular }}}
$$

La problemática de lanzar el proceso de muestreo mediante una señal de pulsos, reside en que el convertidor A/D requiere un tiempo considerable para detectar el pulso externo, lanzar la conversión y proporcionar la muestra. Esto limita la frecuencia máxima de la señal de pulsos que suele ser muy inferior a la frecuencia de muestreo temporal del convertidor.

Si se pretende estudiar los fenómenos de la combustión, la frecuencia de muestreo mínima debe ser de $12 \mathrm{kHz}$ para cubrir todo el ancho de banda hasta un máximo de 6 $\mathrm{kHz}$ [52]. La tabla (3.3) presenta la resolución angular, requerida para este objetivo, en función del régimen de giro. Dichas resoluciones aseguran el adecuado registro de los fenómenos a estudiar, dejando la exactitud de los análisis función del buen referenciado angular respecto al punto muerto superior $(P M S)$ del motor.

\begin{tabular}{|c|c|}
\hline $\begin{array}{c}\text { Régimen de giro } \\
{[\mathrm{rpm}]}\end{array}$ & $\begin{array}{c}\text { Resolución } \\
\text { angular }\left[{ }^{\circ}\right]\end{array}$ \\
\hline 500 & 0.25 \\
\hline 1000 & 0.5 \\
\hline 2500 & 1.25 \\
\hline 5000 & 2.5 \\
\hline
\end{tabular}

Tabla 3.3: Resolución angular requerida para estudios de combustión $\left(f_{m_{\max }}=12 \mathrm{kHz}\right)$.

En el presente trabajo, a la hora del estudio de fenómenos de combustión, se opta por el uso de una resolución angular comprendida entre 0.2 y $0.5^{\circ}$ para abarcar todo el campo de regímenes. Aun que, como se resume en la ilustración de la figura (3.10), la elección de la frecuencia de muestreo depende del uso que se le va a dar a la presión en cámara 
como se comentó anteriormente. Las frecuencias de la figura (3.10) son recomendadas para los estudios experimentales en la investigación de motores de combustión interna alternativos.

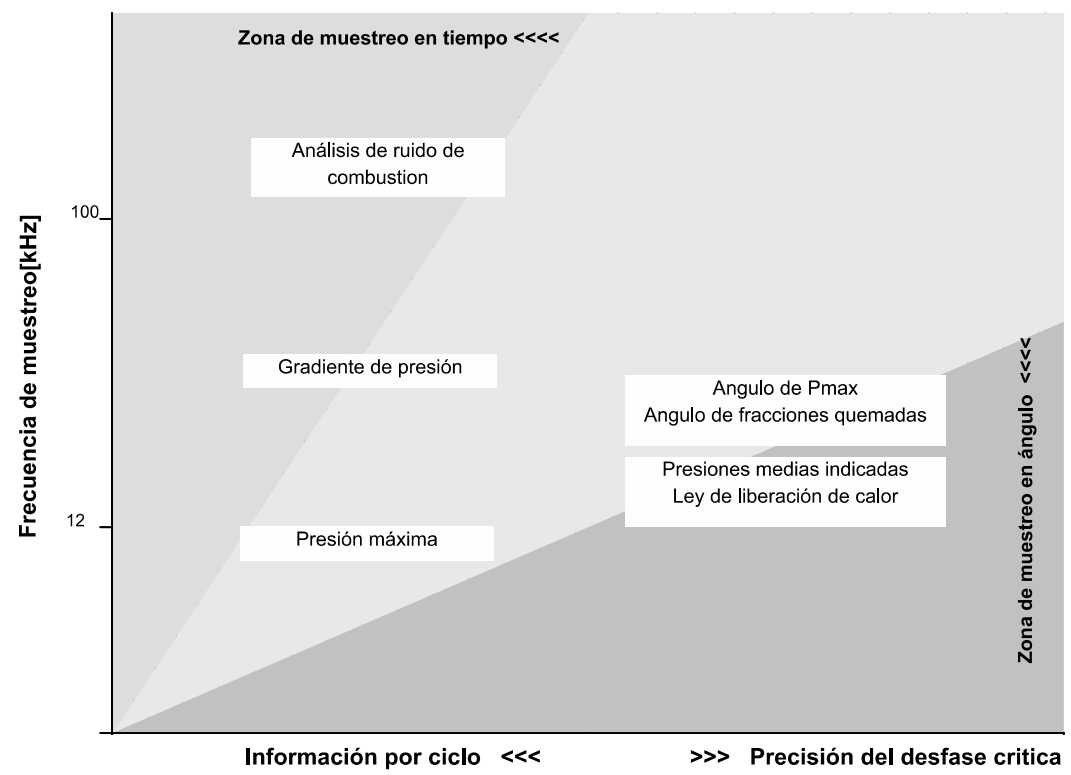

Figura 3.10: Frecuencia de muestreo según el uso de la señal de presión en cámara

En los motores de serie actuales, la referencia angular viene determinada por el sensor de régimen de giro instalado sobre una rueda dentada (generalmente de 60 dientes menos dos que corresponden a un ángulo determinado para el fasado del punto muerto superior relativo a un cilindro). En este caso, la resolución angular máxima es de $6^{\circ}$. Para cumplir los requisitos recomendados de la frecuencia de muestreo, se tiene que estimar el ángulo de giro entre dos pulsos consecutivos de la señal de régimen de giro originados por la rueda dentada, mediante una de las dos formas siguientes:

- Si se hace un post-tratamiento de los datos, se recomienda interpolar el ángulo de giro entre dos pulsos seguidos usando la ecuación 3.3.11.

- Si se requiere la referencia angular instantáneamente, se aconseja extrapolar respeto a valores anteriores del ángulo de giro tomando en cuenta tanto el régimen instantáneo de giro como la aceleración del cigüeñal ya que, por las características de los procesos en los motores, el régimen de giro es variable a lo largo de un ciclo de motor.

Este principio es el usado por el codificador angular, que consiste en una rueda dentada, generalmente 360 dientes y un sensor óptico que detecta el paso de los dientes. La 
resolución en este caso es de $1^{\circ}$ de giro. Sin embargo, la electrónica de estos codificadores angulares permite obtener mayor precisión estimando el ángulo de giro entre dientes y generando más pulsos eléctricos. 


\section{Bibliografía del capítulo}

[1] J.B. Heywood. Internal combustion engine fundamentals. McGraw-Hill,Inc. ISBN 0-07-100499-8, 1988.

[2] J. Martín. Aportación al diagnóstico de la combustión en motores Diesel de inyección directa. Tesis Doctoral, Universidad Politécnica de Valencia, 2007.

[3] F. Payri y J.M. DESANTES. Motores de combustión interna alternativos. isbn: 978-84-291-4802-2, 2011.

[4] J. Martín. Diagnóstico de la combustión en motores diésel de inyección directa. ISBN: 978-84-291-4717-9, 2012.

[5] M. Lapuerta, O. Armas, y J.J. Hernández. "Diagnosis of di diesel combustion from in-cylinder pressure signal by estimation of mean thermodynamic properties of the gas". Applied Thermal Engineering, 19:513-529, 1999.

[6] C. Fenollosa. Aportación a la descripción fenomenológica de proceso de combustión por difusión diesel. Tesis Doctoral, Universidad Politécnica de Valencia, 2003.

[7] J.M. Desantes, J. Galindo, C. Guardiola, y V. Dolz. "Air mass flow estimation in turbocharged diesel engines from in-cylinder pressure measurement". Experimental Thermal and Fluid Science, 34:37-47, 2009.

[8] J.A. Gatowski, E.N. Balles, K.M. Chun, J.A.F.E. Nelson, E. hian, y J.B Heywood. "Heat release analysis of engine pressure data". SAE paper, (841359), 1984.

[9] O. Ulrico, R. Wlodarczyk, y M.T. Wlodarczyk. "High-accuracy low-cost cylinder pressure sensor for advanced engine controls". SAE paper, (2001-01-0991), 2001.

[10] O. Armas. Diagnostico experimental del proceso de combustión en motores Diesel de inyección directa. Tesis Doctoral, Universidad Politécnica de Valencia, Valencia, Spain, 1998. 
[11] M. Morishita y T. Kushiyama. "An improved method for determining the tdc position in a pv-diagram”. SAE paper, (980625), 1998.

[12] D.R. Lancaster, R.B. Krieger, y J.H. Lienesch. "Measurements and analysis of engine pressure data". SAE paper, (750026), 1975.

[13] A. J. Torregrosa, A. Broatch, X. Margot, V. Marant, y Y. Beauge. "Combustion chamber resonances in direct injection automotive diesel engines: a numerical approach”. International Journal of Engine Research, 5(1):83-91, 2004.

[14] A. J. Torregrosa, A. Broatch., J. Martín, y L Monelletta. "Combustion noise level assessment in direct injection diesel engines by means of in-cylinder pressure components". Measurement Science and Technology, 18(7):2131-2142, 2007.

[15] M.F.J. Brunt, H. Rai, y A.L. Emtage. "The calculation of heat release energy from engine cylinder pressure data”. SAE paper, (981052), 1998.

[16] M.F.J. Brunt y C.P. Kieron. "Calculation of heat release in direct injection diesel engines”. SAE paper, (1999-01-0187), 1999.

[17] S. Leonhardt, N. Müller, y R. Isermann. "Methods for engine supervision and control based on cylinder pressure information". IEEE/ASME Transactions on Mechatronic, 4:235-245, 1999.

[18] R. Müller, M. Hart, A. Truscott, A. Noble, G. Krötz, M. Eickhoff, C. Cavalloni, y M. Gnielka. "Combustion pressure based engine management system". SAE paper, (2000-01-0928), 2000.

[19] P. Yoon, S. Parkand M. Sunwoo, I. Ohm, y K.J. Yoon. "Closed-loop control of spark advance and air-fuel ratio in si engines using cylinder pressure". SAE paper, (2000-01-0933), 2000.

[20] Y. Shimasaki, M. Kobayashi, H. Sakamoto, M. Ueno, M. Hasegawa, S. Yamaguchi, y T. Suzuki. "Study on engine management system using chamber pressure sensor integrated with spark plug”. SAE paper, (2004-01-0519), 2004.

[21] M. Beasley, R. Cornwell, P. Fussey, R. King, A. Noble, T. Salamon, y A. Truscott. "Reducing diesel emissions dispersion by coordinated combustion feedback control". SAE paper, (2006-01-0186), 2006.

[22] I. Fuji, S. Yagi, M. Kawai, y H. Yoshikawa. "Mbt control utilizing crank angle of maximum combustion pressure". SAE paper, (890759), 1989.

[23] Y. Hata, K. Ikeura, T. Morita, y T. Abo. "Engine control system using a cylinder pressure sensor". IEE PROCEEDINGS, 136, 1989.

[24] M. Hasegawa, Y. Shimasaki, S. Yamaguchi, M. Kobayashi, H. Sakamoto, N. Kitayama, y T. Kanda. "Study on ignition timing control for diesel engines using in-cylinder pressure sensor". SAE paper, (2006-01-0180), 2006. 
[25] H.L. Husted, D. Kruger, G. Fattic, G. Ripley, y E. Kelly. "Cylinder pressure-based control of pre-mixed diesel combustion”. SAE paper, (2007-01-0773), 2007.

[26] E. Corti, D. Moro, y L. Solieri. "Real-time evaluation of imep and rohr-related parameters". SAE paper, 2007-24-0068(2007-24-0068), 2007.

[27] M.F.J. Brunt y G.G. Lucas. "The effect of crank angle resolution on cylinder pressure analysis". SAE paper, (910041), 1991.

[28] H.S. Rai, M.F.J. Brunt, y C.P. Loader. "Quantification and reduction of imep errors resulting from pressure transducer thermal shock in a si engine". SAE paper, (199901-1329), 1999.

[29] R.H. Kuratle y B. Märki. "Influencing parameters and error sources during indication on internal combustion engines". SAE paper, (920233), 1992.

[30] J. Worm. "An evaluation of several methods for calculating transient trapped mass with emphasis on the "delta p" approach". SAE paper, (2005-01-0990), 2005.

[31] R. Douglas, R.J. Kee, y B.P. Carberry. "Analysis of chamber pressure data in two stroke engines”. SAE paper, (972792), 1997.

[32] R.S. Davis y G.J. Paterson. "Cylinder pressure data quality checks and procedures to maximize data accuracy". SAE paper, (2006-01-1346), 2006.

[33] V. Macian. Contribución al estudio de la influencia del movimiento del ruido sobre la dispersión cíclica y su caracterización. Tesis Doctoral, Universidad Politécnica de Valencia, 1984.

[34] F.V. Tinaut. Contribución al estudio del proceso de combustión en motores de encendido por compresión de inyección directa. Tesis Doctoral, Universidad Politécnica de Valencia, 1986.

[35] E. Oliver. Estudio de sistemas de adquisición y tratamiento de datos en motores de combustión interna alternativos. Tesis Doctoral, Universidad Politécnica de Valencia, Valencia, 1991.

[36] F. Payri, S. Molina, J. Martín, y O. Armas. "Influence of measurement errors and estimated parameters on combustion diagnosis". Applied Thermal Engineering, 26:226-236, 2006.

[37] M. Lapuerta, O. Armas, y V. Bermúdez. "Sensitivity of diesel engine thermodynamic cycle calculation to measurement errors and estimated parameters". Applied Thermal Engineering, 20:843861, 2000.

[38] B. Tormos. Diagnóstico de Motores diésel mediante el análisis del aceite usado. REVERTÉ, S. A., 2005. 
[39] E. Rosseel, R. Sierens, y R.S.G. Baert. "Evaluating piezo-electric transducer response to thermal shock from in-cylinder pressure data". In SAE Conference, Detroit, 1999, 1999.

[40] M.T. Wlodarcyk. "High accuracy glow plug-integrated cylinder pressure sensor for closed loop engine control". SAE paper, (2006-01-0184), 2006.

[41] R. A. Atkins, J. H. Gardner, W. N. Gibler, C. E. Lee, M. D. Oakland, M. O. Spears, V. P. Swenson, H. F. Taylor, J. J. McCoy, y G. Beshouri. "Fiber-optic pressure sensors for internal combustion engines". Applied Optics, 33:1315-1320, 1994.

[42] M.T. Wlodarczyk, T. Poorman, L. Xia, J. Arnold, y T. Coleman. "Incylinder fiberoptic pressure sensors for monitoring and control of diesel engines". SAE paper, (981913):36-42, 1998.

[43] M. Komachiya, H. Sonobe, S. Oho, M. Kurita, T. Nakazawa, y T. Sasayama. "Multiplex in-cylinder pressure measurement utilizing an optical fiber with specific refractive-index composition”. Applied Optics, 35 - 7:1143-1150, 1996.

[44] M. Wendeker y T. Kaminski. "Development of a fiber-optic sensor for the measurement of dynamic cylinder pressure in spark ignition engine". Sensors, IEEE, (2005):8, 2005.

[45] J. Tichý, J. Erhart, E. Kittinger, y J. Prívratská. Fundamentals of piezoelectric sensorics: mechanical, dielectric, and thermodynamical properties of piezoelectric materials. Springer, Berlin ;:London, 2010.

[46] G. Gautschi. Piezoelectric sensorics: force, strain, pressure, acceleration and acoustic emission sensors, materials and amplifiers. Engineering online library. Springer, 2002.

[47] D.T. Hountalas y A. Anestis. "Effect of pressure transducer position on measured cylinder pressure diagram of high speed diesel engines". Energy Conversion and Management, 39:589-607., 1998.

[48] A. Van Aken. "Adapter errors in indicator diagrams of combustion engines". IMechE, 191,8/77:125-134, 1977.

[49] S.C. Homsy y A. Atreya. "An experimental heat release rate analysis of a diesel engine operating under steady conditions". SAE paper, (970889), 1997.

[50] G.H. Neo y N. Collings. "Pressure data analysis of formula one racing engines". SAE paper, (970061), 1997.

[51] A. Arnau Vives. Piezoelectric Transducers and Applications. Springer, 2nd ed. edition, Noviembre 2010. 
[52] F. Payri, A. Broatch, B. Tormos, y V. Marant. "New methodology for in-cylinder pressure analysis in direct injection diesel engines application to combustion noise". Measurement Science and Technology, 16:540-547, 2005.

[53] J. Tichy y G.Gautschi. Piezo-Elektrische Meßtechnik. Springer, 1980.

[54] A. Antoniou. Digital Signal Processing: Signals, Systems, and Filters. McGraw-Hill Professional, Octubre 2005.

[55] P.R. Bevington y D. Robinson. Data Reduction and Error Analysis for the Physical Sciences. McGraw-Hill, 3rd edition, 2002.

[56] M.J. Stas. "An universally applicable thermodynamic method for tdc determination”. SAE paper, (2000-01-0561), 2000. 

Capítulo 4

\section{Fuentes de información experimental y teórica.}




\section{Índice}

4.1. Motores y salas de ensayo. Descripción general. . . . . . . . . . . 77

4.2. Sistema de medida de las variables instantáneas. . . . . . . . . 80

4.3. Determinación de la ley de liberación de calor. . . . . . . . . . 82

4.3.1. Parámetros del motor . . . . . . . . . . . . . . . . . . 83

4.3.2. Ecuación de los gases perfectos . . . . . . . . . . 85

4.3.3. Balance energético . . . . . . . . . . . . . . . 86

4.3.4. Balance másico . . . . . . . . . . . . . . . . . . . 87

4.3.5. Parámetros de interés para el desarrollo de la tesis. . . . . . . 88

Bibliografía del capítulo . . . . . . . . . . . . . . . 93 
El presente apartado no pretende hacer una descripción exhaustiva de la peculiaridades de las instalaciones experimentales empleadas, sino una breve vista general de los sistemas empleados. Especialmente aquellos que tienen efecto directo sobre la medida de la presión en cámara y el diagnóstico de la combustión como se ha indicado en los apartados anteriores.

Además, se pretende en este capítulo aportar una breve descripción de los conceptos teóricos de la herramienta principal de diagnóstico de la combustión empleada en la presente tesis, para la obtención de resultados de referencia que validen las metodologías que serán presentadas más adelante.

\subsection{Motores y salas de ensayo. Descripción general.}

Dado que los ensayos experimentales sobre los motores van a constituir la fuente de información básica para el desarrollo del presente trabajo, se han planteado dichos ensayos de forma que satisfagan los requisitos del modelo de diagnóstico de combustión (CALMEC [1]) empleado para cotejar y validar los resultados finales de la presente tesis. Para ello, y siguiendo la metodología de trabajo expuesta en varios trabajos anteriores ( [2-5] entre otros), se han utilizado para cada ensayo variables registradas por la propia ECU, variables medias (de temperaturas, gastos másicos y presiones medias) registradas por los sistemas de adquisición de datos disponibles en las diferentes salas de ensayo y variables instantáneas, aplicando a todas ellas los criterios de selección del sistema de adquisición de datos discutidos en el capítulo anterior.

En cuanto a los motores diésel utilizados, diferenciamos en dos modalidades principales: motores monocilíndricos y policilíndricos. Los motores monocilíndricos son versiones que reproducen los motores que se fabrican en serie para su uso en automoción. La existencia de un solo cilindro evita la posible aparición de dispersiones entre cilindros, como ocurre en los motores policilíndricos, tanto a nivel de geometrías como a nivel de las condiciones de funcionamiento, y da la posibilidad de tener un sistema de sobrealimentación independiente de la combustión. Esto permite evitar fuentes de error y aclara las relaciones causa-efecto de los fenómenos estudiados. Además, la realización de las medidas sobre un solo cilindro implica una reducción, en parte, de los equipos de medida y una reducción de costes de operación al disminuir los consumos de combustible, refrigerante y energía eléctrica.

Para el desarrollo de la presente tesis, se han utilizado un motor monocilíndrico y tres motores policilíndricos diferentes. Las principales características de cada uno de los motores se recogen en la tabla (4.1).

Cada motor está instalado en una sala de ensayo equipada con un dinamo-freno, cuyas prestaciones son acordes con la potencia del motor a ensayar. Dichos dinamo-frenos se controlan electrónicamente, permitiendo así realizar tanto ensayos en puntos estabilizados como dinámicos (tanto variaciones de carga como aceleraciones de motor o simulación de ciclos en carretera).

Los motores son instrumentados, sin alterar sus prestaciones, con sensores acordes con las exigencias de la investigación. Combinando así la información extraída 


\begin{tabular}{lccccc} 
Parámetro & & Monocilíndrico & M9R & DW10 & DW12 \\
\hline \hline $\mathbf{N}^{\mathbf{0}}$ de Cilindros & {$[-]$} & 1 & 4 & 4 & 4 \\
Cilindrada total & {$[l]$} & 2.2 & 2 & 2 & 2.1 \\
Carrera & {$[\mathrm{mm}]$} & 96 & 90 & 88 & 96 \\
$\begin{array}{l}\text { Diámetro } \\
\text { Relación de } \\
\text { compresión }\end{array}$ & {$[\mathrm{mm}]$} & 85 & 84 & 85 & 85 \\
$\begin{array}{l}\text { Rango de } \\
\text { regímenes }\end{array}$ & & 18 & 15.6 & 16 & 18 \\
Rango de Par & $\mathrm{rpm}$ & $1000-4500$ & $1000-4500$ & $780-4500$ & $900-5000$ \\
\hline
\end{tabular}

Tabla 4.1: Principales características de los motores ensayados.

de las ECUs. Además, cada sala de ensayo está equipada con los sensores y actuadores necesarios para medir y controlar todas las variables de interés. Se asegura de esta manera una alta precisión y repetitividad de los ensayos al emplear circuitos independientes de acondicionamiento de combustible, aire, agua y lubricante, instrumentados adecuadamente y controlados mediante algoritmos de control adaptados a cada circuito de acondicionamiento.

La figura (4.1) presenta un ejemplo de la instrumentación de las salas de ensayo utilizadas para el desarrollo de la presente tesis. En concreto se representa el caso de una sala de ensayos para motores monocilíndricos.

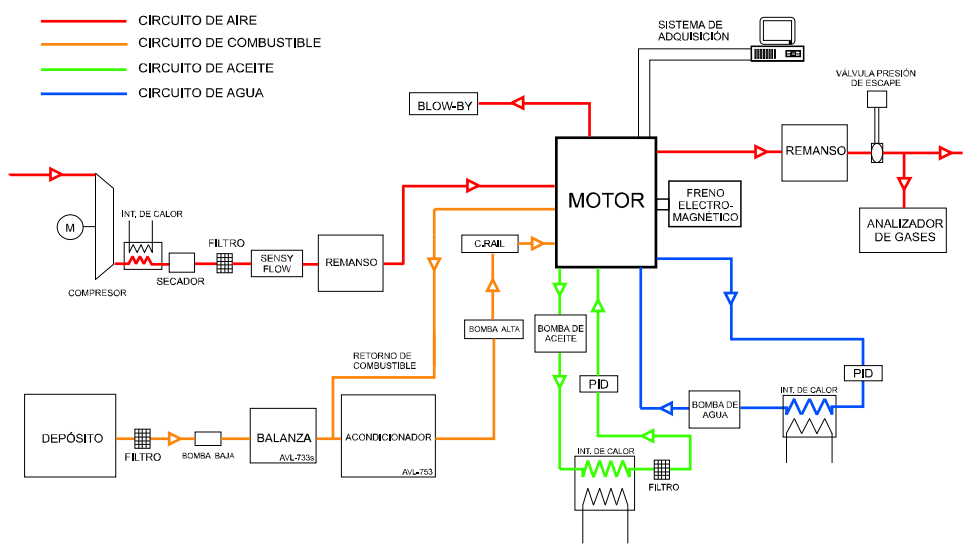

Figura 4.1: Esquema de la sala de ensayos para motores monocilíndricos. 
Las variables registradas para determinar la ley de liberación de calor, con la máxima precisión, para cada ensayo han sido las siguientes:

Régimen de giro medio. Se mide, con una precisión de $\pm 1 \mathrm{rpm}$, mediante un sensor magnético montado sobre el eje del freno que va acoplado al cigüeñal. Dicho freno es el encargado de absorber la potencia del motor en el caso de ensayos en combustión y de hacer girar al motor en ensayos con el motor arrastrado. Las consignas de régimen de giro son alcanzadas mediante el variador de frecuencias del freno, siendo la dinámica de alcance de las consignas, básicamente, función de la carga y del régimen de giro del motor [6].

Par: Se mide, con una resolución $\pm 0.1 \mathrm{Nm}$, mediante una célula de carga colocada en acople entre el eje del freno y el cigüeñal, Un proceso de calibración periódica, mediante pesas calibradas, permite lecturas fiables de este parámetro.

Gasto de aire: medido con un sensor de hilo caliente y corregido en función de la temperatura y la presión.

Gasto de combustible: Se mide mediante una balanza. El error de los equipos de medida suele ser inferior al $1 \%$ para puntos estacionarios.

Gasto de blow-by. Se mide mediante una tobera calibrada o un medidor de vórtice forzado. El error de dichos equipos suele ser inferior al $\pm 5 \%$.

Presiones medias del gas en el colector de admisión y en el colector de escape. Se miden mediante sensores piezoresistivos refrigerados, si el punto de medida lo exige, logrando una precisión de \pm 10 mbar.

Temperaturas medias del gas en el colector de admisión y de escape, del refrigerante, del aceite lubricante, del combustible y otras temperaturas son medidas mediante termopares. El equipo de medida alcanza una precisión de $\pm 1^{\circ} \mathrm{C}$.

Variables de la ECU: presiones, temperaturas, gastos y caudales medidas por la ECU con los propios sensores del motor además de variables de control que aplica la propia ECU como el número, puntos y duración de las inyecciones.

Variables instantáneas: con una frecuencia de muestro adecuada para cada régimen de giro y una resolución óptima, definida en el capítulo anterior, se han registrado, entre otras variables, las siguientes:

- El pulso de comando del inyector para determinar la tasa de inyección a partir de la caracterización previa de los inyectores.

- La señal de la presión en cámara o la señal correspondiente a la derivada de la presión en cámara utilizando la alternativa que será propuesta en el apartado 5.4 del capítulo 5. 


\subsection{Sistema de medida de las variables instantáneas.}

La tabla (4.2) resume el efecto de algunos parámetros del sistema de medida de presión sobre la información obtenida de dicha medida tal y como se ha discutido en el capítulo anterior.

\begin{tabular}{|l|c|c|c|}
\hline \multirow{2}{*}{ Variable } & \multicolumn{3}{|c|}{ Parámetros del sistema de adquisición } \\
\cline { 2 - 4 } & $\begin{array}{c}\text { Sensibilidad } \\
\text { del sensor }\end{array}$ & $\begin{array}{c}\text { Sensibilidad } \\
\text { térmica }\end{array}$ & $\begin{array}{c}\text { Frecuencia } \\
\text { de muestreo }\end{array}$ \\
\hline \hline Presión máxima & Baja & Media & Baja \\
\hline ángulo de la presión máxima & Baja & Alta & Alta \\
\hline Diagrama $\log (p) / \log (V)$ & Baja & Alta & Baja \\
\hline Presiones medias indicadas & Alta & Alta & Media \\
\hline Gradiente de presiones & Alta & Baja & Alta \\
\hline \hline Ruido de combustión & Alta & Baja & Muy Alta \\
\hline \hline Ley de liberación de calor & Alta & Media & Media \\
\hline $\begin{array}{l}\text { SOC, EOC, Ángulos de } \\
\text { Fracciones quemadas }\end{array}$ & Alta & Media & Alta \\
\hline Fracciones quemadas & Alta & Media & Media \\
\hline Temperaturas & Baja & Media & Baja \\
\hline Emisiones & Baja & Baja & Media \\
\hline
\end{tabular}

Tabla 4.2: Sensibilidad de la información obtenida a partir de la presión en cilindro en función de algunos parámetros del sistema de adquisición de datos

Siendo la ley de liberación de calor la fuente principal que se va a utilizar para la validación de los resultados de la presente tesis, y considerando los criterios de selección del sensor y sistema de adquisición de datos para las señales instantáneas comentados en el apartado 3.3 del capítulo anterior, se ha optado por el uso de sensores piezoeléctricos y el sistema de adquisición de datos mostrados en las tablas 4.3 y 4.4 sucesivamente.

\begin{tabular}{lcccc} 
Sensor & & Kistler 6055B & Kistler 6057A & AVL GU13P \\
\hline \hline Sensibilidad & $\mathrm{pC} / \mathrm{bar}$ & 20 & 15 & 15.53 \\
Rango & $\mathrm{bar}$ & $0-250$ & $0-250$ & $0-200$ \\
Frecuencia natural & $\mathrm{kHz}$ & 130 & 130 & 130 \\
Linealidad & $\%$ & 0.4 & 0.6 & 0.3 \\
Rango de temperaturas & ${ }^{\circ} \mathrm{C}$ & $-50-400$ & $-50-350$ & $-50-400$ \\
Sensibilidad térmica & $\%$ & 2 & 1.8 & 2 \\
Motor & & DW10-DW12 & Monocilíndrico & M9R \\
\hline
\end{tabular}

Tabla 4.3: Sensores piezoeléctricos utilizados para la medida de presión en cámara. 


\begin{tabular}{lc} 
Nombre & Yokogawa DL716 \\
\hline \hline Número de canales & 16 \\
Resolución & $16 \mathrm{bits}$ \\
Frecuencia de muestreo máxima & $100 \mathrm{kHz}$ \\
Resolución angular empleada & $0.5^{\circ}$ \\
\hline
\end{tabular}

Tabla 4.4: Sistema de adquisición de señales instantáneas. 


\subsection{Determinación de la ley de liberación de calor.}

En la figura (4.2) se muestra un esquema general del proceso de diagnóstico de la combustión a partir de la presión en cámara (CALMEC) elaborado y validado por el grupo de investigación [1]. Donde, a partir de la medida de la presión en cámara acondicionada, variables medias que reflejan las condiciones de funcionamiento del motor (temperaturas, presiones medias, gastos, etc.) y constantes relacionadas con la geometría del motor y con el ajuste del modelo, se obtienen resultados medios ( $p m i$, ángulos de quemado de fracciones del combustible, SoC, EoC, etc.) y resultados instantáneos, principalmente la ley $(F Q L)$ o la tasa de liberación de calor $(d F Q L)$ en función del ángulo de giro del motor.

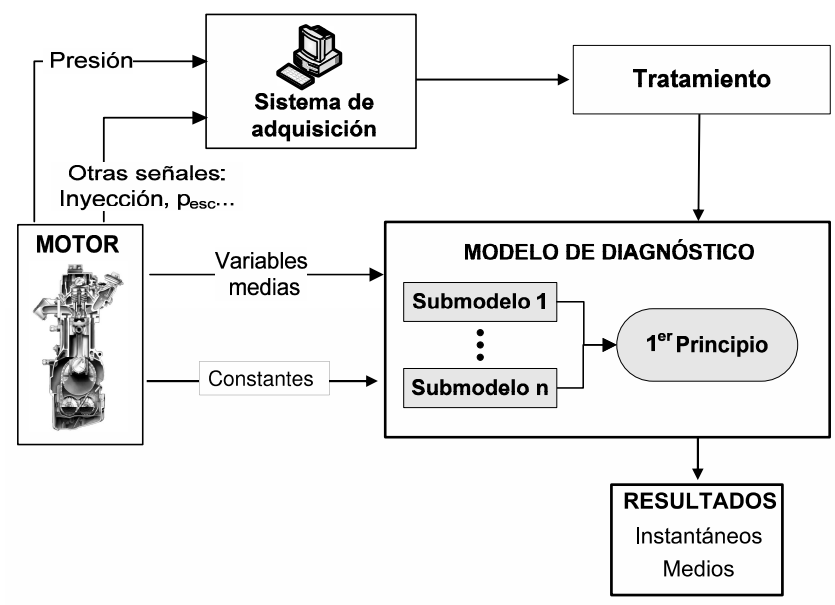

Figura 4.2: Esquema general del proceso de diagnóstico de la combustión a partir de la presión en cámara.

El modelo de diagnóstico de la combustión se basa en las siguientes hipótesis:

1. Presión uniforme en la cámara.

2. Existen tres especies en la cámara: aire, combustible gaseoso y productos quemados estequiométricamente.

3. La mezcla de gases tiene comportamiento de gas ideal.

4. La energía interna se calcula mediante correlaciones en función de la temperatura y la composición instantánea.

5. Se utiliza la temperatura media de la cámara (modelo de una zona) o de cada zona (modelo de 2 zonas) para el cálculo de la energía interna sensible. 
6. La cámara de combustión durante el ciclo cerrado es un sistema abierto debido al blow-by y la inyección de combustible (en MEC y GDI).

7. Existe transmisión de calor por convección a las paredes de la cámara.

8. Se considera que los elementos en movimiento se deforman por presión e inercia.

\subsubsection{Parámetros del motor}

El volumen instantáneo, de la ecuación (4.3.1), se calcula a partir de la geometría del mecanismo biela-manivela, en función del ángulo de giro del cigüeñal y teniendo en cuenta la relación de compresión del motor $\left(r_{c}\right)$ :

$$
V=V_{c c}+\frac{\pi \cdot D^{2}}{4}\left[L_{\text {máx }}-L\right]+\Delta V_{p}+\Delta V_{i}
$$

donde:

$L_{\text {max }}-L \quad$ se calcula resolviendo el mecanismo biela-manivela:

$$
L_{\text {max }}-L=\left(L_{b}+S / 2\right)-S / 2 \cdot\left(\cos (\alpha)+\sqrt{1 / \lambda^{2}-\operatorname{sen}^{2}(\alpha)}\right)
$$

$V_{C C} \quad$ es el volumen de la cámara de combustión calculado a partir de $r_{c}=\frac{V_{d}+V_{C C}}{V_{C C}}$

$V d=\frac{\pi \cdot D^{2}}{4} \cdot\left(L_{\max }-L\right)$ es el volumen instantáneo desplazado por el mecanismo de biela-manivela.

Los volúmenes encerrados por la geometría del cilindro se ven afectados por las deformaciones.

siendo:

$\Delta V_{p} \quad$ es el incremento de volumen debido a la deformación por presión:

$\Delta V_{p}=K_{\text {def }} \cdot \frac{\pi \cdot D^{2}}{4} \cdot \frac{p}{E_{\text {acero }}} \cdot\left(\frac{D}{d_{\text {bulon }}}\right)^{2} \cdot\left(h_{\text {cab }}+L_{b}+\frac{S}{2}\right)$

$\Delta V_{i} \quad$ es el incremento o decremento del volumen por las fuerzas de inercia sobre los elementos móviles:

$$
\Delta V_{i}=K_{\text {def }} \cdot \frac{m \cdot a}{E_{\text {acero }}} \cdot\left(\frac{D}{d_{\text {bulon }}}\right)^{2} \cdot\left(h_{\text {cab }}+L_{b}+\frac{S}{2}\right)
$$


Los parámetros de la geometría del motor necesarios para el cálculo del volumen son, por lo tanto, los siguientes:

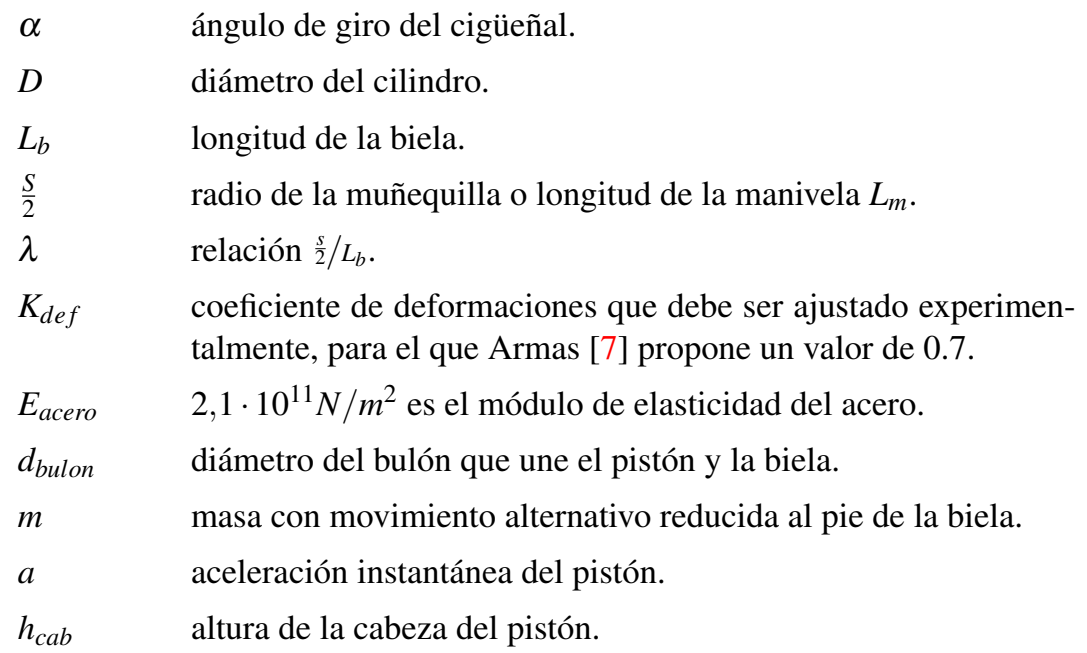

Además de estos parámetros, es imprescindible determinar la posición del PMS respecto a la presión máxima registrada, ya que, fenómenos como deformaciones mecánicas, dilataciones térmicas de los materiales y/o holguras entre piezas, hacen que la posición angular correspondiente al volumen mínimo en condiciones estáticas y dinámicas no coincidan.

Errores en el calado angular de la presión de $1^{\circ}$ conducen a errores no despreciables en el diagnóstico de combustión y principalmente en el cálculo de la PMI. Para evitar esto, es necesario conocer el desplazamiento del ángulo de volumen mínimo (considerado en este trabajo como el ángulo del PMS) y al que nos referimos como el desfase presión - volumen. En la literatura se han encontrado diversos métodos cuyo objetivo es la determinación de este desfase.

Métodos experimentales, que pueden alcanzar los $0.03^{\circ}$ de resolución, son basados en la introducción de sensores intrusivos cuya precisión depende del posicionamiento correcto del sensor [8].

Los métodos teóricos basados en ensayos de arrastre del motor, calculan, mediante aproximaciones a polinomios o análisis del exponente politrópico, la presión máxima real necesaria para determinar el desfase termodinámico.

Algunos de estos métodos consideran que la posición de la presión máxima en un motor real arrastrado no coincide con el ángulo de volumen mínimo, sino que existe un desfase termodinámico $\left(\triangle \alpha_{t}\right)$ debido principalmente a las pérdidas de calor por transmisión a las paredes $\left(\triangle \alpha_{Q}\right)$ y en menor medida a las pérdidas de masa por el blow-by $\left(\triangle \alpha_{m}\right)$.

En este trabajo, y según Hohenberg [9], dichos desfases pueden calcularse con la siguiente expresión: 


$$
\triangle \alpha_{t}=\triangle \alpha_{Q}+\triangle \alpha_{m}=\left[\frac{Q_{p_{\max }}}{(c p / R)}+\frac{60 \cdot m_{b b_{\max }} \cdot R \cdot T}{2 \pi \cdot N}\right] \cdot \frac{180 \cdot \pi}{\pi \cdot p_{\max } \cdot K_{1}}
$$

siendo:

$$
\begin{array}{ll}
p_{\max } & \text { la presión máxima en }[\mathrm{Pa}] \\
Q_{p_{\max }} & \text { el calor, en }[\mathrm{J} / \mathrm{rad}], \text { transmitido a las paredes en el ángulo de } p_{\max } \\
m_{b b_{p_{\max }}} & \text { el gasto másico en }[\mathrm{kg} / \mathrm{s}] \text { de } b l o w-b y \text { en el ángulo de } p_{\max } \\
N & \text { el régimen de giro del motor en }[\mathrm{rpm}] \\
K_{1} & =\frac{\pi \cdot D^{2} \cdot L_{m}}{4} \cdot(1+L m / L b) \text { en }[\mathrm{m} 3 / \mathrm{rad} 2], \text { un parámetro que depen- } \\
& \text { de de la geometría del motor. }
\end{array}
$$

Con este método se asegura un valor del desfase que puede ser utilizado con bastante precisión para continuar con el desarrollo de la tesis.

\subsubsection{Ecuación de los gases perfectos}

Aplicando la ecuación (4.3.3) de los gases perfectos, puede calcularse la temperatura de la mezcla (aire, combustible y gases quemados), una vez conocida la presión y el volumen de la cámara:

$$
p \cdot V=m_{c} \cdot R_{c} \cdot T
$$

La masa de la mezcla puede calcularse mediante la ecuación (4.3.4) desde el cierre de la válvula de admisión $\left(\alpha_{i n i}=R C A\right)$ hasta el ángulo $\alpha$ :

$$
m_{c}(\alpha)=m_{a}+m_{E G R}+m_{r e s}-m_{c c}+\int_{S O I}^{\alpha} m_{f}-\int_{R C A}^{\alpha} m_{b b}
$$

donde:

$$
\begin{array}{ll}
m_{a} & \text { masa de aire instantánea. } \\
m_{E G R} & \text { masa de gases recirculados (EGR). } \\
m_{r e s} & \begin{array}{l}
\text { masa de gases residuales, estimada mediante un modelo sencillo } \\
\text { de llenado y vaciado. }
\end{array} \\
m_{c c} & \begin{array}{l}
\text { masa de cortocircuito estimada con el mismo modelo que el } \\
\text { utilizado para estimar la } m_{\text {res }} .
\end{array} \\
\int_{S O I}^{\alpha} \dot{m_{f}} & \begin{array}{l}
\text { gasto másico del combustible acumulado desde el inicio de la } \\
\text { inyección (masa de combustible) }
\end{array} \\
\int_{R C A}^{\alpha} \dot{m_{b b}} & \begin{array}{l}
\text { gasto másico acumulado de fugas por blow-by desde el cierre de } \\
\text { admisión. }
\end{array}
\end{array}
$$


La constante específica $R_{c}$ de la ecuación (4.3.3) puede obtenerse en función de la concentración de las especies existentes en la cámara en cada instante mediante la ecuación (4.3.5):

$$
R_{c}=R_{a} Y_{a}+R_{f} Y_{f}+R_{q} Y_{q}
$$

donde:

$R_{i} \quad$ constante específica de cada especie (aire para $i=a$, combustible para $i=f$, productos quemados estequiométricamente para $i=$ $q)$ ). Se calcula como el cociente entre la constante específica de los gases y el peso molecular de cada especie $(i)$.

$Y_{i} \quad$ fracción másica de cada especie (modelo una zona ${ }^{1}$ ).

Del mismo modo, se calculan los calores específicos a volumen constante $\left(c_{v, a}, c_{v, f}\right.$ y $\left.c_{v, q}\right)$ y las energías internas específicas $\left(u_{a}, u_{f}\right.$ y $\left.u_{q}\right)$ que se obtienen por medio de correlaciones polinómicas en función de la temperatura [10].

\subsubsection{Balance energético}

Aplicando el primer principio de la termodinámica a la cámara de combustión, considerada como sistema abierto debido a las fugas por blow-by y al aporte de combustible, el balance energético permite obtener el calor liberado en la combustión. En efecto, la expresión general del primer principio describe la variación de la energía interna de los gases en la cámara como:

$$
\begin{aligned}
\mathrm{d} U_{c} & =-\mathrm{d} Q+\mathrm{d} W+h_{f, i n y} \cdot \mathrm{d} m_{f, i n y}-h_{c} \cdot \mathrm{d} m_{b b} \\
& =-\mathrm{d} Q-p \cdot \mathrm{d} V+h_{f, i n y} \cdot \mathrm{d} m_{f, i n y}-h_{c} \cdot \mathrm{d} m_{b b}
\end{aligned}
$$

donde:

$$
\begin{aligned}
& \mathrm{d} U_{c}=\mathrm{d}\left(m_{c} \cdot u_{c}\right) \text { la variación de la energía interna de los gases en la cámara. } \\
& \begin{aligned}
Q=h_{w} \cdot\left[A_{p}\right. & \left.\left(T_{g}-T_{p}\right)+A_{c u l}\left(T_{g}-T_{c}\right)+A_{c i l}\left(T_{g}-T_{c i l}\right)\right] \text { es el calor transmiti- } \\
& \text { do a las paredes con: } \\
& h_{w}: \text { coeficiente de película, se calcula mediante el modelo de } \\
& \text { Woschni mejorado [5] } \\
& A_{i} \text { y } T_{i}: \text { área y temperatura de pared (p: pistón, cul: culata y cil: } \\
& \text { cilindro) calculadas mediante el modelo nodal [5,7] } \\
& T_{g}: \text { temperatura del gas } \\
p \cdot \mathrm{d} V \quad & \text { es el trabajo. }
\end{aligned}
\end{aligned}
$$

\footnotetext{
${ }^{1}$ El modelo es de una zona si sólo se considera la fase gaseosa, donde el combustible líquido inyectado no se considera a efectos de masa ni energía hasta que se haya evaporado.
} 
$h_{f, i n y} \cdot \mathrm{d} m_{f, i n y}$ es el flujo de entalpía liberado por la masa de combustible inyectada $\left(d m_{f, i n y}\right)$, donde $h_{f, \text { iny }}$ es la entalpía del combustible inyectado. Despreciando el volumen del combustible líquido y asumiendo evaporación y calentamiento instantáneos, $d m_{f, i n y}$ puede ser reemplazada por la variación de masa de combustible evaporada $\left(d m_{f, e v}\right)$ como indica la ecuación (4.3.7).

$h_{c} \cdot \mathrm{d} m_{b b} \quad$ es la energía perdida por fugas de blow-by.

$$
\mathrm{d} U_{c}=-\mathrm{d} Q-p \mathrm{~d} V+h_{f, i n y} \mathrm{~d} m_{f, e v}-h_{c} \mathrm{~d} m_{b b}
$$

\subsubsection{Balance másico}

La variación de la energía interna de los gases en la cámara se puede calcular aplicando el balance másico.

$$
\begin{aligned}
\mathrm{d} U_{c}= & \mathrm{d}\left(m_{a} \cdot u_{a}+m_{f, g} \cdot u_{f, g}+m_{q} \cdot u_{q}\right) \\
= & m_{a} \cdot \mathrm{d} u_{a}+m_{f, g} \cdot \mathrm{d} u_{f, g}+m_{q} \cdot \mathrm{d} u_{q} \\
& +\mathrm{d} m_{a} \cdot u_{a}+\mathrm{d} m_{f, g} \cdot u_{f, g}+\mathrm{d} m_{q} \cdot u_{q}
\end{aligned}
$$

En la ecuación (4.3.8) se aprecia que $\mathrm{d} U_{c}$ se compone de una energía interna sensible, que es debida al cambio de temperatura, y de una energía interna debida al cambio de la composición de los gases por causa de la combustión.

Considerando el modelo de una zona, la variación de la energía interna sensible se plantea mediante la siguiente ecuación:

$$
m_{a} \cdot \mathrm{d} u_{a}+m_{f, g} \cdot \mathrm{d} u_{f, g}+m_{q} \cdot \mathrm{d} u_{q}=m_{c} \cdot c_{v, c} \cdot \mathrm{d} T
$$

La variación de la energía interna por el cambio de la composición, teniendo en cuenta el combustible evaporado y las pérdidas por el blow-by puede expresarse como:

$$
\begin{aligned}
& \mathrm{d} m_{a} \cdot u_{a}+\mathrm{d} m_{f, g} \cdot u_{f, g}+\mathrm{d} m_{q} \cdot u_{q}= \\
& \quad\left[u_{q}-\frac{u_{a}+u_{f, g} \cdot F_{e}}{F_{e}+1}\right] \cdot\left(\mathrm{d} m_{q}+Y_{q} \cdot \mathrm{d} m_{b b}\right)-u_{q} \cdot \mathrm{d} m_{b b}+u_{f, e v} \cdot \mathrm{d} m_{f, e v}
\end{aligned}
$$

donde $F_{e}$ presenta la fracción entre las masas de aire y combustible que se queman en condiciones estequiométricas $\left(F_{e}=\mathrm{d} m_{f, q} / \mathrm{d} m_{a, q}\right)$.

Sustituyendo y reorganizando, la ecuación (4.3.7) queda

$$
\begin{aligned}
& {\left[u_{q}-\frac{u_{a}+u_{f, g} F_{e}}{F_{e}+1}\right] \cdot\left(\mathrm{d} m_{q}+Y_{q} \mathrm{~d} m_{b b}\right)=}
\end{aligned}
$$

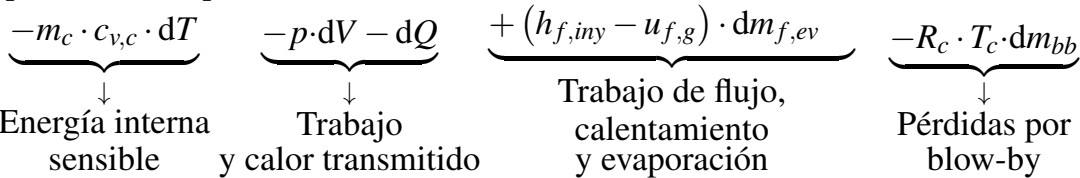


El primer miembro de la igualdad anterior es el calor liberado en la combustión, de modo que puede escribirse como en la ecuación (4.3.12), donde dicho calor es la suma de la variación de la energía interna sensible, la transmisión de calor a las paredes de la cámara, el trabajo de los gases sobre el pistón, la energía asociada al fenómeno de inyección-evaporación del combustible y el trabajo de flujo de las fugas por blow-by.

$$
\mathrm{dFQL}=m_{c} \cdot c_{v, c} \cdot \mathrm{d} T+\mathrm{d} Q+p \cdot \mathrm{d} V-\left(h_{f, i n y}-u_{f, g}\right) \mathrm{d} m_{f, e v}+R_{c} T_{c} \mathrm{~d} m_{b b}
$$

Esta ecuación, representa la formula principal del cálculo de la tasa de liberación de calor que va a ser utilizada para la validación de los resultados de la presente tesis. Si bien, la cantidad de variables que se toman en cuenta para su cálculo y la acumulación de errores en la obtención de cada variable puede sugerir un grado importante de incertidumbres en las tasas calculadas y en definitiva en el diagnóstico de la combustión, el conocimiento de la sensibilidad del modelo de diagnóstico de combustión a la variación de cada variable involucrada adquirido por el grupo de investigación de la CMT, ha permitido optimizar, para cada tipo de motor, el diagnóstico de la combustión resumiendo todo el conocimiento adquirido, en este campo, en el programa llamado CALMEC, cuyos resultados son fiables y serán utilizados como referencia en la presente tesis.

\subsubsection{Parámetros de interés para el desarrollo de la tesis.}

Probablemente la información más valiosa que se puede obtener a partir de la señal de presión en cámara y parámetros del motor es la ley de liberación de calor. Dicha ley, junto con información del proceso de inyección, permite obtener con detalle el tiempo de retraso y las características de las tres fases de la combustión definidas para motores diésel (combustión premezclada, combustión por difusión y combustión por difusión tardía) [11,12]. Se comentarán a continuación se comentarán estas tres fases:

Fase I (combustión premezclada). Localizada entre el inicio de la combustión (SoC) y el primer mínimo local de la tasa de liberación de calor. Esta fase ocurre muy rápidamente, tiene un carácter fuertemente no estacionario y es donde se produce la oxidación del combustible que se ha mezclado con el aire durante el tiempo de retraso.

Fase II (combustión por difusión). La ley de liberación de calor durante esta fase está controlada principalmente por el proceso de mezcla del combustible evaporado con el aire en el interior de la cámara de combustión. Generalmente se asume que la combustión se realiza en condiciones localmente estequiométricas, o próximas a ella, en términos de oxígeno y combustible. Durante esta fase la estructura de la llama alcanza un período de cuasi-estacionariedad, que se mantiene mientras se siga inyectando combustible. Dicha fase dura hasta el final de la inyección (EoI).

Fase III (combustión por difusión tardía o lenta). Consiste en un decrecimiento progresivo de la ley de liberación de calor causado por el deterioro del proceso de mezcla aire/combustible, acompañado de generación de energía cinética turbulenta 


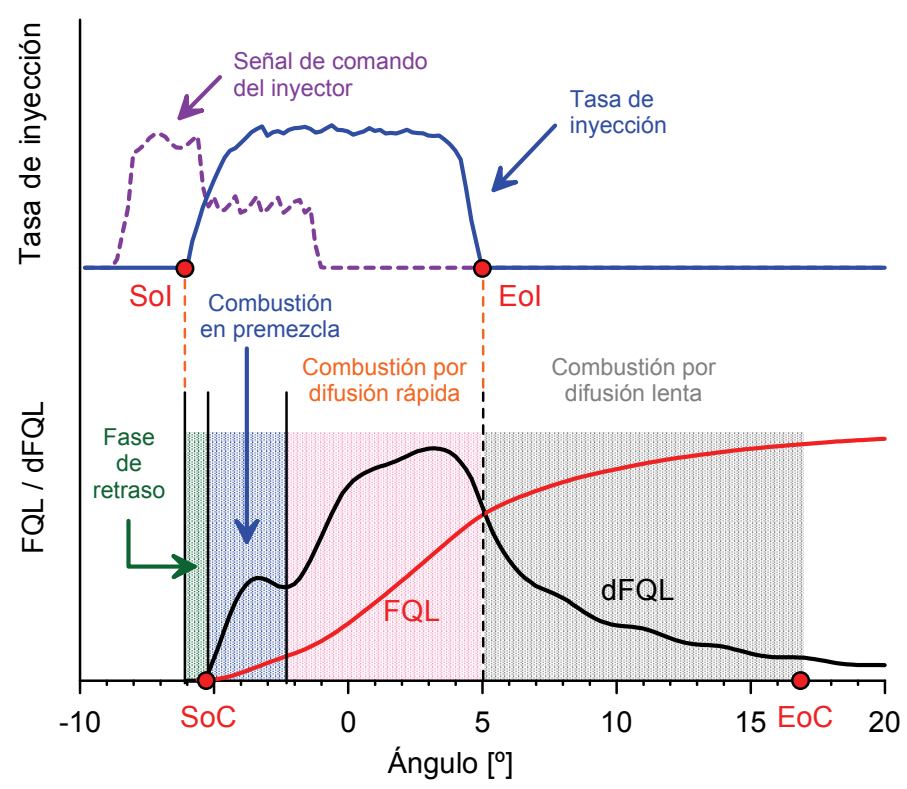

Figura 4.3: Fases de la combustión

en el interior de la cámara de combustión dado que el pistón se suele encontrar en su fase de expansión.

Antes de todo, conviene acotar los parámetros más importantes de la ley de combustión, que sus evaluaciones en tiempo real pueden ser de gran utilidad. En este sentido, no se puede despreciar la ley de liberación de calor instante por instante (magnitudes en Julios $/^{\circ}$ ), ni el total de las fracciones de calor liberado en cada momento o ángulo de giro (ángulo de quemado del 10,50,90...\% del combustible), pero, los tres siguientes parámetros son de especial interés:

El ángulo de inicio de combustión "Start of Combustion"(SoC): que corresponde al ángulo de inicio de la combustión. A partir de este parámetro, y dado el ángulo de inicio de la inyección (Start of Injection (SoI)), se obtiene el tiempo de retraso que es debido a dos fenómenos simultáneos:

- Retraso físico $(\backsim 10 \%)$. El tiempo necesario para la vaporización y homogeneización del combustible. Es función de las características del chorro (presión de inyección, tobera), propiedades del combustible (viscosidad), densidad y movimiento del aire en la cámara.

- Retraso químico ( $\backsim 90 \%)$. Representa el tiempo en el que se realizan las prereacciones necesarias para el inicio de la combustión. Es función de las propiedades 
del combustible (índice de cetano), temperatura y presión del aire en cámara (a mayor temperatura y presión al final de la compresión menos tiempo de retraso) y choque del chorro con las paredes.

El ángulo de final de la combustión "End of Combustion"(EoC) que permite definir la duración de la combustión.

El ángulo de máxima tasa de liberación de calor ángulo de dFQLmax donde la velocidad de la combustión alcanza su máximo.

El SoC tiene especial importancia en el control de la combustión de los motores diésel, ya que permite obtener directamente el tiempo de retraso que debería ser lo más corto posible. Un tiempo de retraso largo, y en condiciones de bajas cargas y con el motor frío, ocasiona golpeteo o "knock", lo que causa importantes pérdidas de calor, de rendimiento e incluso averías del motor.

En cuanto a las metodologías de detección de los parámetros definidos anteriormente, si bien el punto de máxima liberación de calor ( $d F Q L \max$ ) es fácil de detectar, los criterios para determinar los puntos de inicio y final de la combustión son cuestionables.

En la literatura [11], se estima el ángulo del SoC como aquel ángulo de giro en el que se ha quemado una determinada cantidad del combustible. Dicha cantidad se suele tomar entre el 1 y el $10 \%$ del combustible total introducido independientemente del número de inyecciones o combustiones a lo largo del mismo ciclo. Otro método cada vez más extendido ( [13-16]) se basa en el cociente de presiones (presión en combustión/ presión en arrastre), considerando el SoC como el punto en el que la presión en combustión es superior a la presión en arrastre (estimada), o bien relacionando mediante ecuaciones lineales los ángulos en que se alcanzan determinados valores del cociente de presiones con el SoC estimado por otras metodologías.

Otros se basan en el diagrama $\log (p) / \log (V)$ y consideran como inicio de la combustión el instante donde el diagrama se despega de la recta asíntota que marca la fase de expansión [17].

En cuanto al EoC, no hay un consenso claro para determinar dicho parámetro. Por lo general, se considera el EoC como el ángulo en el que se ha liberado más del $90 \%$ de la energía del combustible total inyectado. Algunos autores consideran el $90 \%$, otros el $95 \%$, incluso algunos lo consideran como el $99 \%$. Por lo general, a partir del $90 \%$ del calor liberado, la $F Q L$ toma una forma casi plana debido a la baja eficiencia del proceso de la oxidación. Eso hace, que la diferencia entre el ángulo donde se haya quemado el $90 \%$ del combustible y donde se haya quemado el $99 \%$ pueda alcanzar varias decenas de grados. En la presente tesis, se considerará el EoC como el ángulo de giro donde se alcanza el $95 \%$ del total del calor liberado en la combustión principal.

En lo que sigue, se considerará como uno de los métodos más robustos, el basado en el análisis de la curva de $d F Q L$, considerando (para el caso teórico) el SoC como el ángulo en que la $d F Q L$ toma valores superiores a 0 . Se toma esta opción, ya que si bien para el caso de combustiones fuertes la mayoría de los métodos son validos, en caso de combustiones débiles es de suma dificultad asegurar con cualquiera de los métodos basados en la presión en cámara la localización del SoC. Ejemplo de ello, y como se 
mostrara a continuación, es el caso de inyecciones múltiples, donde pueden solaparse las combustiones, o para inyecciones muy tempranas en condiciones que dificultan las combustiones piloto. En estos casos, además de herramientas de análisis se requiere el ojo experto u otras herramientas para el análisis de la combustión.

Para la validación de los resultados de la presente tesis, se calcula mediante CALMEC la ley de liberación de calor y, una vez obtenida esta ley, se localiza directamente el ángulo de dFQLmax. En cuando al inicio de la combustión, se resuelve de la forma siguiente:

- No se toma en cuenta la parte negativa de la $\mathrm{d} F Q L$.

- Se divide la $\mathrm{d} F Q L$ en tramos definidos por dos valles, o pasos por cero, intercalados por un pico, solapando tramos con área total inferior a un $2 \%$ del área total del tramo adjunto. Obteniendo así, varios mínimos locales (dos por tramo), de los cuales se elegirán los correspondientes al SoC de cada combustión. Para llevar a cabo esta elección, partiendo de los puntos de inyección se estima el retraso teórico del auto-encendido mediante la correlación propuesta por [18] en función de la presión y temperatura media : $\tau_{\text {delay }}=0,0043 \cdot \exp \left(\frac{8870}{\bar{T}}\right) \cdot \bar{p}^{-1,258}$.

- En las zonas de influencia, determinadas por dos inicios de la inyección más el retraso teórico del auto-encendido correspondiente (]$\alpha_{\text {iny }, i}+\tau_{\text {delay }, i}, \alpha_{\text {iny }, i+1}+$ $\tau_{\text {delay }, i+}[$ ), se consideran los tramos anteriormente definidos como posibles partes de combustión. De estos tramos, se desestiman aquellos cuyo calor liberado sea inferior al $6 \%$ de la energía teórica liberada por la cantidad de combustible inyectada en dicha inyección. De esta forma, se considera el SoC como el primer mínimo local del tramo definitivo correspondiente a cada inyección y el EoC como el final del tramo o el correspondiente al $95 \%$ del total del calor liberado en caso de la combustión principal.

En el ejemplo de la figura (4.4), se aprecia que para el caso de la combustión principal la determinación del SoC es directa, sin embargo existen dos opciones para el SoC de la combustión piloto (presentadas en puntos negros). El programa CALMEC puede desestimar el primer punto puesto que el posible calor que se hubiese liberado por la $\mathrm{dFQL}$ entre los dos puntos seria insignificante frente al total, como se refleja en la ley de liberación de calor $F Q L$ adimensional presentada en la misma figura.

Este método ha sido comprobado en múltiples motores y en un gran número de condiciones de funcionamiento de motores y aporta resultados altamente coherentes pese a la sensibilidad del diagnóstico de la combustión a los parámetros y las hipótesis de cálculo [5]. Sin embargo, hay que resaltar que las tolerancias empleadas en la detección del SoC pueden alterar significativamente los resultados, principalmente para los SoC correspondientes a combustiones débiles. En la figura (4.4), por ejemplo, hay aproximadamente $7^{\circ}$ de diferencia entre considerar el SoC en un punto o en otro. Además, al no tener en cuenta los valores negativos, diferencias del orden de $1^{\circ}$ pueden afectar a la determinación final de los parámetros de la combustión. 


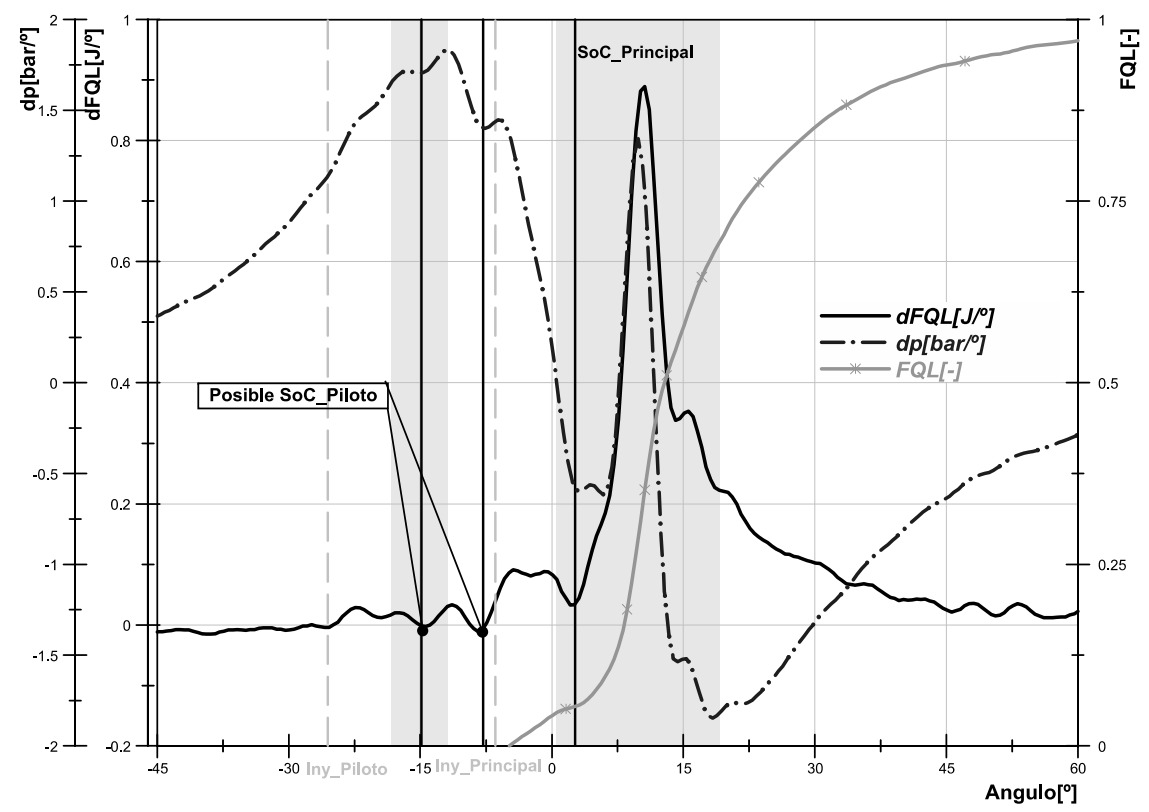

Figura 4.4: Localización del SoC a partir de la dFQL (Motor DW12 a 3500rpm,16.3Nm) 


\section{Bibliografía del capítulo}

[1] F. Payri, S. Molina, J. Martín, y O. Armas. "Influence of measurement errors and estimated parameters on combustion diagnosis". Applied Thermal Engineering, 26:226-236, 2006.

[2] V. Macian. Contribución al estudio de la influencia del movimiento del ruido sobre la dispersión cíclica y su caracterización. Tesis Doctoral, Universidad Politécnica de Valencia, 1984.

[3] M. Lapuerta. Un modelo de combustión fenomenológico para un motor Diesel de inyección directa rápido. Tesis Doctoral, Universidad Politécnica de Valencia, 1988.

[4] C. Fenollosa. Aportación a la descripción fenomenológica de proceso de combustión por difusión diesel. Tesis Doctoral, Universidad Politécnica de Valencia, 2003.

[5] J. Martín. Aportación al diagnóstico de la combustión en motores Diesel de inyección directa. Tesis Doctoral, Universidad Politécnica de Valencia, 2007.

[6] Carlos Guardiola. Detección y compensación de irregularidades de inyección a través de la medida del régimen instantáneo del turbogrupo. Temas avanzados en motores de combustión interna. Editorial Reverté, S.A., 2005.

[7] O. Armas. Diagnostico experimental del proceso de combustión en motores Diesel de inyección directa. Tesis Doctoral, Universidad Politécnica de Valencia, Valencia, Spain, 1998.

[8] E. Oliver. Estudio de sistemas de adquisición y tratamiento de datos en motores de combustión interna alternativos. Tesis Doctoral, Universidad Politécnica de Valencia, Valencia, 1991.

[9] G. Hohenberg. Experimentelle Erfassung der Wandwarme in Kolbenmotoren (Experimental acquisition of the wall heat in piston engines). Tesis Doctoral, Technical University of Graz, Graz, Austria, 1980. 
[10] M. Lapuerta, O. Armas, y J.J. Hernández. "Diagnosis of di diesel combustion from in-cylinder pressure signal by estimation of mean thermodynamic properties of the gas”. Applied Thermal Engineering, 19:513-529, 1999.

[11] J.B. Heywood. Internal combustion engine fundamentals. McGraw-Hill,Inc. ISBN 0-07-100499-8, 1988.

[12] F. Payri y J.M. DESANTES. Motores de combustión interna alternativos. isbn: 978-84-291-4802-2, 2011.

[13] S. Leonhardt, N. Müller, y R. Isermann. "Methods for engine supervision and control based on cylinder pressure information". IEEE/ASME Transactions on Mechatronic, 4:235-245, 1999.

[14] M. C. Sellnau, F. A. Matekunas, P. A. Battiston, C.Chang, y D.R. Lancaster. "Cylinder-pressure-based engine control using pressure-ratio-management and lowcost non-intrusive cylinder pressure sensors". SAE paper, (2000-01-0932), 2000.

[15] R. Reitz y J. von der Ehe. "Use of in-cylinder pressure measurement and the response surface method for combustion feedback control in a diesel engine". IMechE, 220 Part D, 2006.

[16] M. Yoon, K. Lee, M. Sunwoo, y B. Oh. "Cylinder pressure based combustion phasing control of a CRDI diesel engine”. SAE paper, (2007-01-0772), april 2007.

[17] R. Douglas, R.J. Kee, y B.P. Carberry. "Analysis of chamber pressure data in two stroke engines”. SAE paper, (972792), 1997.

[18] J.M. García. Aportación al estudio del proceso de combustión turbulenta de chorros en motores diesel de inyección directa. Tesis Doctoral, Universidad Politécnica de Valencia, 2004. 
Capítulo 5

Aporte al tratamiento de la presión en cámara para el diagnóstico de la combustión. 


\section{Índice}

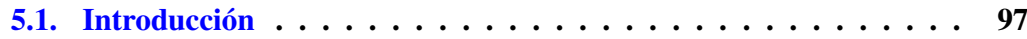

5.2. Etapa 1. Promediado ................. 98

5.2.1. Propuesta para definir el número de ciclos a promediar . . . . 105

5.3. Etapa 2. El filtrado digital de la señal de presión . . . . . . . . 111

5.3.1. Métodos experimentales para determinar los parámetros del filtro . . . . . . . . . . . . . . . . . . 114

5.3.2. Propuesta para determinar los parámetros del filtro . . . . . 115

5.3.3. Propuesta para el filtrado de la señal de presión en cilindro en tiempo real. . . . . . . . . . . . . . . . . . . 118

5.4. Propuesta para la medida de la derivada de la presión en cámara 119

Bibliografía del capítulo . . . . . . . . . . . . . 123 


\subsection{Introducción}

En los capítulos anteriores, se ha tratado la sensibilidad de la medida del sensor respeto al sistema de adquisición utilizado. Se han definido los requisitos de dicho sistema en función de la aplicación final que se le pretende dar a la medida de presión en cámara. Sin embargo, hay que hacer constar que de los posibles ruidos que pueden estar en una medida, no todos son debidos al sistema de adquisición de datos, de hecho, aun cuando un motor este funcionando de forma estable en unas determinadas condiciones operativas, pequeñas variaciones en los procesos termofluidodinámicos hacen que en la cámara no se produzca una evolución de la presión perfectamente cíclica.

Esta dispersión tiene su origen en variaciones de la cantidad del combustible suministrado, del punto de inyección o encendido y del llenado y movimiento del aire en la cámara. Esto lleva a la necesidad de medir varios ciclos de presión consecutivos, ya que uno solo cualquiera elegido al azar puede no ser representativo del funcionamiento estacionario del motor.

Sin embargo, esta alternativa no es viable cuando se pretende hacer estudios donde la presión en cilindro cambia entre un ciclo y otro, como en el caso de los transitorios. En estos casos, la dispersión es debida a cambios reales en el punto de funcionamiento del motor, pues el tránsito de un punto a otro significa cambios significativos en gasto de combustible, masa de aire, puntos de inyección, etc. Por lo tanto, solo se procederá a la obtención de un ciclo representativo de varios ciclos consecutivos para estudios estacionarios.

En general, el tratamiento de la medida de la presión en cámara, una vez registrada, consta de 3 etapas:

1. Promediado: que trata de obtener un ciclo representativo de varios ciclos medidos, haciendo un promediado matemático u otras alternativas como se detallara más adelante.

2. Filtrado: aplicando filtros para eliminar ruidos y/o información no deseada.

3. Referenciado: que permite obtener presiones absolutas reales a partir de las lecturas registradas de la señal del sensor piezoeléctrico. Los métodos de referenciado y la sensibilidad del diagnóstico de la combustión a los errores de referenciado se han estudiado ampliamente en varios trabajos ( [1-4] entre otros) y no serán discutidos en la presente tesis.

A continuación se detallarán de forma particular las dos primeras etapas comentadas anteriormente. 


\subsection{Etapa 1. Promediado}

La ecuación (5.2.1) representa el promediado matemático de una serie de valores determinados:

$$
\overline{p_{\alpha}}=\frac{1}{n_{c}} \cdot \sum_{k=1}^{n_{c}} p_{\alpha, k}
$$

donde:

$$
\begin{array}{ll}
\overline{p_{\alpha}} & \text { la presión media de la muestras en } \alpha . \\
n_{c} & \text { el número de ciclos a promediar. } \\
p_{\alpha, k} & \text { la muestra } \alpha \text { de la presión medida en el ciclo } k .
\end{array}
$$

La presión media obtenida cumple con dos criterios:

- Bajo error típico en la medida.

- Filtrado de la señal.

En primer lugar, y desde el punto de vista estadístico y según el teorema del límite central, si de cualquier población $\left(\left\{p_{\alpha, 1}, p_{\alpha, 2}, . ., p_{\alpha, n_{c}}, . ., p_{\alpha, N}\right\}\right)$ se extraen muestras aleatorias del mismo tamaño $n_{c}\left(\left\{p_{\alpha, 1+j}, p_{\alpha, 2+j}, \ldots, p_{\alpha, n_{c}+j}\right\}_{j \in\left[0, N / n_{c}\right]}\right)$, al aumentar el número de muestras sus medias se distribuyen normalmente, con media y un error típico definidos como:

$$
\text { err }_{\text {tip }}=\frac{\sqrt{\sum\left(\overline{p_{\alpha}}-p_{\alpha, k}\right)^{2}}}{n_{c}}=\frac{\text { desviacióntípica }}{\sqrt{n_{c}}}
$$

El error típico representa la desviación del promedio de las muestras tomadas respeto a la media de la población. Es decir, si se considera la media de la población como el valor más preciso de la presión en un punto dado, al promediar $n_{c}$ muestras de esta población el error cometido se aproxima al error típico con un nivel de confianza dado (ya que se desconoce la media de la población). Por lo tanto, es natural que al aumentar el tamaño de las muestras $\left(n_{c}\right)$ el error sea menor ya que la media de la muestra se aproximará más a la media de la población. Si $n_{c}$ es muy grande, el error tiende a cero y, en este caso, la media de la muestra converge a la media de la población [5].

En definitiva, se puede afirmar que el promediado reduce la amplitud ruido con una razón equivalente a la raíz cuadrada de $n_{c}$, considerando el ruido en este caso como el error típico de la medida.

Por otra parte, el promediado actúa como un filtro de medias móviles, considerado como un filtro de paso bajo que permite eliminar de la señal componentes de muy baja frecuencia. Para evaluar este filtro habría que recurrir al análisis de la señal en dominio de frecuencias.

En efecto, dada una serie de muestras de la señal de presión en cámara $\left(\left\{p_{\alpha, 1}, p_{\alpha, 2}, . ., p_{\alpha, n_{c}}, . ., p_{\alpha, N}\right\}\right)$, la ecuación (5.2.1) puede reescribirse de la forma siguiente: 


$$
\overline{p_{\alpha}}=\frac{1}{n_{c}} \cdot \sum_{k=1}^{n_{c}} p_{\alpha, k}=\sum_{k=1}^{n_{c}} \frac{1}{n_{c}} \cdot p_{\alpha, k}=\sum_{k=-\infty}^{+\infty} h_{k} \cdot p_{\alpha, k}
$$

Donde:

$$
h_{k}= \begin{cases}\frac{1}{n_{c}} & \text { para } k=1 \cdots n_{c} \\ 0 & \text { para otros casos }\end{cases}
$$

Además la ecuación (5.2.2) sugiere que el promediado es la convolución matemática $\left((p * h)_{i}\right)$ de la serie de muestras tomadas $p$ y el filtro $h$.

El teorema de convolución [6] afirma que la transformada de Fourier de una convolución de dos funciones es el producto punto a punto de las transformadas de las funciones. En otras palabras, la convolución en el dominio temporal es equivalente al producto punto a punto en el dominio espectral o de frecuencias.

Para el caso de señales discretas, se emplea la transformada discreta de Fourier (TDF) "discrete Fourier transform " unidimensional expresada como:

$$
F(h)=\frac{1}{N} \cdot \sum_{x=0}^{N-1} f(x) \cdot e^{-\frac{2 \cdot \pi \cdot j \cdot x \cdot h}{N}}
$$

Donde:

$F(h) \quad$ es la TDF de la función temporal discreta $f(x)$ compuesta por una secuencia de $N$ números complejos que representan el peso de contribución a $f(x)$ de una función periódica (exp $\left[\frac{2 \cdot \pi \cdot j \cdot x \cdot h}{N}\right]=$ $\left.\cos \left(\frac{2 \cdot \pi \cdot x \cdot h}{N}\right)+j \cdot \operatorname{sen}\left(\frac{2 \cdot \pi \cdot x \cdot h}{N}\right)\right)$, en dominio de frecuencias.

$j \quad$ es la unidad imaginaria $(\sqrt{-1})$.

$x \quad$ el índice del elemento correspondiente a $f(x)$ (muestra: $0,1,2 \ldots, N$ 1).

$h \quad$ el índice del armónico, o factor de multiplicación de la frecuencia fundamental, entre 0 y $N-1$.

$N \quad$ el número de elementos que forman $f(x)$ (número total de muestras).

$e^{-\frac{2 \cdot \pi \cdot j}{N}} \quad$ la raíz N-ésima de la unidad.

El módulo de $F(u)$ presenta el espectro de Fourier como el representado en la figura (5.2).

Del mismo modo, se define la inversa de la transformada de Fourier como:

$$
f(x)=\sum_{h=0}^{N-1} F(h) \cdot e^{\frac{2 \cdot \pi \cdot j \cdot x \cdot h}{N}}
$$


Para el filtro $h_{k}$, que se asemeja al promediado de la señal (ecuación (5.2.3)), la TDF es:

$$
H[f]=\frac{\sin \left(\pi \cdot f \cdot n_{c}\right)}{n_{c} \cdot \sin (\pi \cdot f)}
$$

Donde la frecuencia $f$ varía entre 0 y la mitad de la frecuencia del ciclo del motor $\left(f_{0}\right)$.

La figura (5.1) representa la respuesta en dominio frecuencia del filtro de medias móviles para diferentes números de ciclos a promediar $(n c)$, donde el eje de magnitud representa la ganancia del filtro y el eje de frecuencias relativas representa el cociente entre la frecuencia de la señal y la frecuencia de muestreo. Se aprecia que este filtro tiene una mala atenuación de las frecuencias que se pretenden eliminar lo que hace de él un filtro no óptimo para eliminar frecuencias bajas y, por lo tanto, fuerza al uso de filtros más avanzados en el tratamiento de la señal.

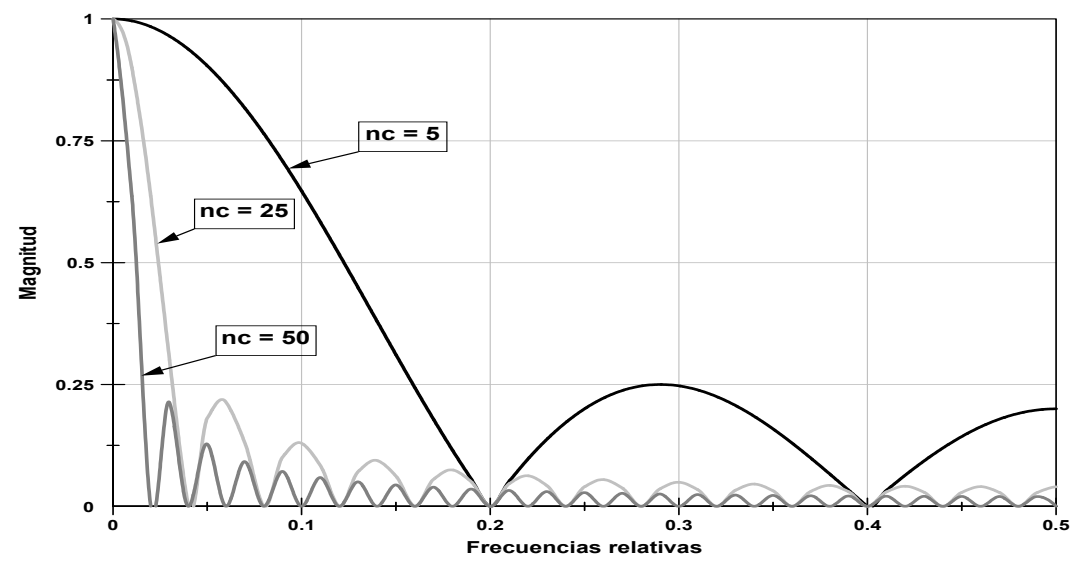

Figura 5.1: Respuesta en frecuencia de filtros de media móvil con diferentes números de ciclos a promediar.

En el caso práctico, el espectro de la señal (figura (5.2)) muestra que tanto en arrastre como en combustión, los armónicos del ciclo medio tienen un modulo mucho mayor que los que no corresponden al ciclo medio.

Para la figura (5.2) se ha tomado una medida de 25 ciclos consecutivos de presión en cámara con una frecuencia de muestreo angular de $0.5^{\circ}$ obteniendo 1440 muestras por ciclo. Para cada ángulo de giro se calcula la media de las presiones mediante ecuación (5.2.1) obteniendo el ciclo promedio.

En la figura (5.2) se aprecia que los armónicos múltiples del número de ciclos muestreados $\left(n_{c}\right)$ tienen magnitudes similares a las del ciclo promedio. La explicación radica en la multiplicación de la transformada de Fourier del filtro $(H(f)$ de la ecuación (5.2.6)) por la transformada de la serie de muestras correspondientes a cada ángulo de giro. 


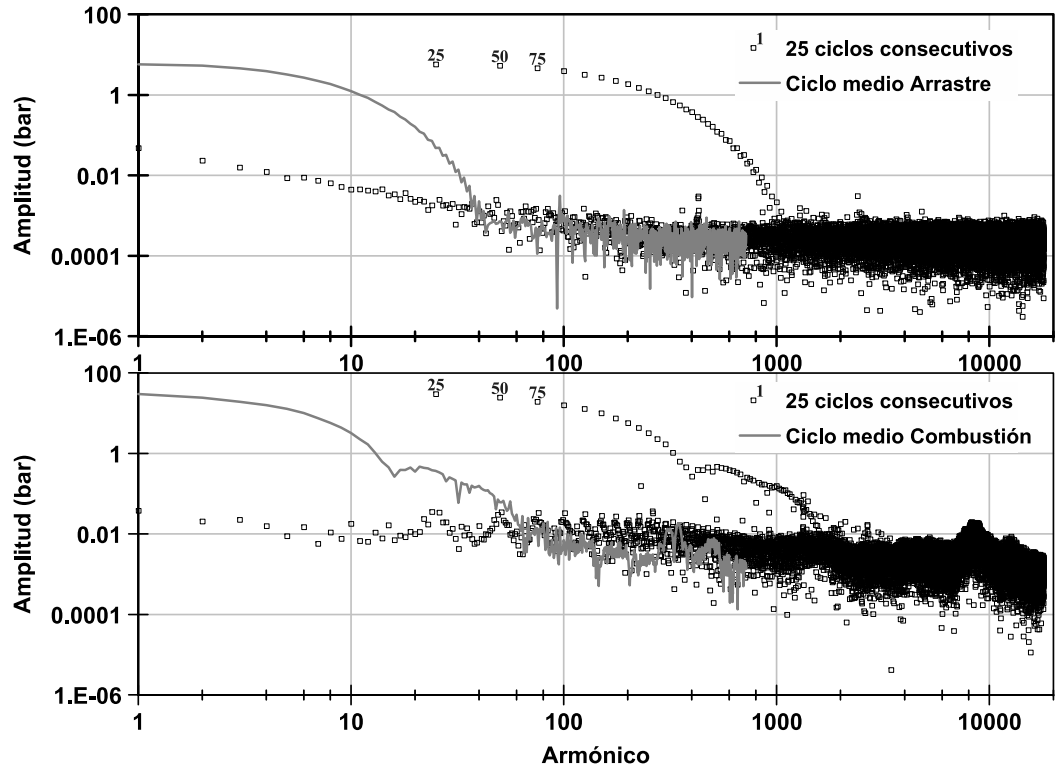

Figura 5.2: Espectro de señal medida de presión en arrastre (arriba) y combustión (abajo) 
Martín [4] argumenta que la frecuencia fundamental del ciclo medio es $n_{c} \cdot f_{0} \mathrm{y}$, por lo tanto, los armónicos correspondientes a frecuencias múltiplos de $n_{c} \cdot f_{0}$ serán armónicos tanto del ciclo medio como de la señal con $n_{c}$ ciclos. Eso es cierto siempre que la señal sea periódica, es decir, para valores $p_{k}=p_{k+n_{p c}}$. Por este motivo, en el trabajo de Martín [4], para garantizar que se cumpla la condición de periodicidad se aplica una ventana a la señal completa. Para ello se ajusta el nivel de la señal de modo que se minimice $p_{k}^{2}+p_{k+n_{p c}}^{2}$ $\mathrm{y}$, a continuación, se multiplica por una ventana de periodo $1 / f_{0}$ y valor 1 salvo en los extremos, donde se emplean dos rampas sinusoidales que van de 0 a 1 al inicio y de 1 a 0 al final.

Sin embargo, si no se considera que la señal es completamente periódica, se propone considerar la señal medida como una función discreta bidimensional $f(x, y)$ donde $y$ representa el número de ciclo y $x$ representa el número de la muestra o el ángulo de giro .

Aplicar la transformada discreta de Fourier bidimensional (TDF $b$ ) se corresponde a calcular la ecuación siguiente:

$$
F(u, v)=\frac{1}{N \cdot M} \sum_{x=0}^{M-1} \sum_{y=0}^{N-1} f(x, y) \cdot e^{-2 \pi j\left(\frac{u \cdot x}{M}+\frac{v \cdot y}{N}\right)}
$$

La transformada inversa se calcula mediante:

$$
f(x, y)=\sum_{u=0}^{M-1} \sum_{v=0}^{N-1} F(u, v) \cdot e^{2 \pi j\left(\frac{u \cdot x}{M}+\frac{v \cdot y}{N}\right)}
$$

Donde los incrementos de muestreo en el dominio espacial y de frecuencia están relacionados por: $\Delta u=\frac{1}{M \cdot \Delta x} \quad$ y $\quad \Delta v=\frac{1}{N \cdot \Delta y}$

Además, la característica de la separabilidad de la TDF permite calcular la TDF de una función bidimensional como una combinación de dos transformadas de Fourier discretas unidimensionales, calculando primero una TDF sobre una variable y aplicando al resultado de nuevo la TDF sobre la otra variable $[6,7]$.

$$
\begin{gathered}
F(u, v)=\frac{1}{n_{p c}} \sum_{y=0}^{n_{p c}-1} F(u, y) \cdot e^{-2 \pi j\left(\frac{u \cdot y}{n_{p c}}\right)} \\
F(u, y)=\frac{1}{n_{c}} \sum_{x=0}^{n_{c}-1} f(x, y) \cdot e^{-2 \pi j\left(\frac{u \cdot x}{n_{c}}\right)}
\end{gathered}
$$

Por lo tanto, si una función puede tener forma unidimensional (un conjunto de $n_{c} \cdot n_{p c}$ elementos sucesivos) y forma bidimensional (un conjunto de $n_{p c}$ valores repetidos $n_{c}$ veces), la TDF será equivalente a la TDF $b$ reconstruyendo la matriz de resultados TDF $b$, es decir:

Si $f_{u}(x)=f_{b}\left(x_{1}, y_{1}\right) \Leftrightarrow F_{u}(h)=F_{b}(u, v)$

A modo de ejemplo, para $\left(x=x_{1}, y_{1}=0\right)$ la conclusión anterior es inmediata y $(h=u$ $, v=0)$. 
Sin embargo, en el caso general consideramos el cambio de variable $x$ por: $x=$ $x_{1}+n_{p c} \cdot y_{1}$ con $\left(x_{1}=\left(0,1, \ldots, n_{p c}-1\right) ; y_{1}=\left(0,1, \ldots, n_{c}-1\right)\right)$. Claramente, para cada valor de $x$ existe un único par de valores $\left(x_{1}, y_{1}\right)$ que cumpla la igualdad $x=x_{1}+n_{p c} \cdot y_{1} \mathrm{y}$, por lo tanto, se puede comprobar la siguiente igualdad:

$$
\frac{1}{n_{c} \cdot n_{p c}} \cdot \sum_{x=0}^{n_{c} \cdot n_{p c}-1}(\cdots)=\frac{1}{n_{c} \cdot n_{p c}} \cdot \sum_{x_{1}=0}^{n_{p c}-1} \sum_{y_{1}=0}^{n_{c}-1}(\cdots)
$$

Por lo tanto, la TDF expresada en la ecuación (5.2.4) adoptará la forma:

$$
F(h)=\frac{1}{n_{c} \cdot n_{p c}} \cdot \sum_{x_{1}=0}^{n_{p c}-1} \sum_{y_{1}=0}^{n_{c}-1} f\left(x_{1}, y_{1}\right) \cdot e^{-2 \pi j\left(\frac{h \cdot\left(x_{1}+n_{p c} \cdot y_{1}\right)}{n_{c} \cdot n_{p c}}\right)}
$$

Por otra parte, el armónico $h$ puede, a su vez, reconstruirse en forma bidimensional considerando $h=n_{c} \cdot u+v$, entonces:

$$
F(h)=\frac{1}{n_{c} \cdot n_{p c}} \cdot \sum_{x=0}^{n_{p c}-1} \sum_{y=0}^{n_{c}-1} f\left(x_{1}, y_{1}\right) \cdot e^{-2 \pi j\left(\frac{\left(n_{c} \cdot u+v\right) \cdot\left(x_{1}+n_{p c} \cdot y_{1}\right)}{n_{c} \cdot n_{p c}}\right)}
$$

Desarrollando:

$$
\begin{aligned}
F_{u}(h) & =\frac{1}{n_{c} \cdot n_{p c}} \cdot \sum_{x=0}^{n_{p c}-1} \sum_{y=0}^{n_{c}-1} f\left(x_{1}, y_{1}\right) \cdot e^{-2 \pi j\left(\frac{n_{c} \cdot u \cdot n_{p c} \cdot y_{1}+n_{c} \cdot u \cdot x_{1}+v \cdot n_{p c} \cdot y_{1}+v \cdot x_{1}}{n_{c} \cdot n_{p c}}\right)} \\
& =\frac{1}{n_{c} \cdot n_{p c}} \cdot \sum_{x=0}^{n_{p c}-1} \sum_{y=0}^{n_{c}-1} f\left(x_{1}, y_{1}\right) \cdot e^{-2 \pi j\left(\frac{n_{c} \cdot u \cdot n_{p c}+1}{n_{c} \cdot n_{p c}}\right)} \cdot e^{-2 \pi j\left(\frac{n_{c} \cdot u \cdot x_{1}+v \cdot n_{p c} \cdot y_{1}+v \cdot x_{1}}{n_{c} \cdot n_{p c}}\right)} \\
& =\frac{1}{n_{c} \cdot n_{p c}} \cdot \sum_{x=0}^{n_{p c}-1} \sum_{y=0}^{n_{c}-1} f\left(x_{1}, y_{1}\right) \cdot e^{-2 \pi j\left(\frac{x_{1}}{n_{p c}} \cdot\left(u+\frac{v}{n_{c}}\right)+\frac{y_{1} \cdot v}{n_{c}}\right)}
\end{aligned}
$$

En definitiva:

$$
F_{u}\left(h=n_{c} \cdot u+v\right)=F_{b}\left(u+\frac{v}{n_{c}}, v\right)
$$

del mismo modo se puede comprobar que si $f\left(x=n_{c} \cdot x_{1}+y_{1}\right)=f\left(x_{1}, y_{1}\right)$, entonces:

$$
F_{u}\left(h=u+n_{c} \cdot v\right)=F_{b}\left(u, v+\frac{u}{n_{p c}}\right)
$$

Una medida de la presión en cámara compuesta de $n_{c}$ ciclos, cada uno de $n_{p c}$ muestras, se puede considerar como una función bidimensional $p(x, y)$ donde $x$ es el ciclo e $y$ la muestra correspondiente a un ángulo de giro: 


$$
p(x, y)=\left(\begin{array}{cccc}
p_{0,0} & p_{1,0} & \cdots & p_{n_{p c}-1,0} \\
p_{0,1} & p_{1,1} & \cdots & p_{n_{p c}-1,1} \\
\vdots & \vdots & \cdots & \vdots \\
p_{0, n_{c}-1} & p_{1, n_{c}-1} & \cdots & p_{n p_{c}-1, n_{c}-1}
\end{array}\right)
$$

Entonces, la ecuación (5.2.7) toma la siguiente forma:

$$
P(u, v)=\frac{1}{n_{p c} \cdot n_{c}} \sum_{x=0}^{n_{c}-1} \sum_{y=0}^{n_{p c}-1} p(x, y) \cdot e^{-2 \pi i\left(\frac{u . x}{n_{c}}+\frac{v . y}{n_{p c}}\right)}
$$

Por tanto, la transformada de la matriz $p(x, y)$ se puede realizar calculando, en una primera etapa, la transformada unidimensional de cada una de sus columnas (presiones correspondientes a un ángulo de giro constante) y posteriormente, calcular la transformada a cada una de las filas de la matriz $P(u, y)$ obtenida en la primera etapa.

La primera fila de la matriz $P(u, y)$ corresponde a $P(u=0, y)$. Sustituyendo en la ecuación (5.2.10):

$$
P(0, y)=\frac{1}{n_{c}} \sum_{x=0}^{n_{c}-1} p(x, y) \cdot e^{0}=\frac{1}{n_{c}} \sum_{x=0}^{n_{c}-1} p(x, y) \quad y=\left(0,1, \ldots, n_{p c}-1\right)
$$

Es decir, cada elemento de la primera fila de la matriz $P(u, y)$ representa cada uno de los elementos del ciclo medio calculado mediante promedio matemático (5.2.1). Eso es así puesto que el primer armónico de la TDF de una señal siempre corresponde con la media de la señal completa (nivel de continua). Por lo tanto, la TDF de la primera fila de la matriz $P(u, y)$ es equivalente a la TDF del ciclo medio.

La TDF de la ecuación (5.2.14) corresponde a la $P(0, v)$. Teniendo en cuenta la relación (5.2.12), los armónicos correspondientes a $P(0, v)$ son equivalentes a los armónicos $h=n_{c} \cdot v$ de la TDF de una medida de presión compuesta por $n_{c}$ ciclos consecutivos. Además, dichos armónicos componen la TDF del promedio de la señal de presión.

La TDF bidimensional es ampliamente usada en el tratamiento de imágenes [8]. Para nuestro caso, algunas del las ventajas que puede aportar esta herramienta pueden ser:

- Disminución de la carga computacional: la TDF necesita $\left(n_{p c} \cdot n_{c}\right)^{2}$ multiplicaciones complejas y $\left(n_{p c} \cdot n_{c}\right)^{2}-1$ sumas complejas. La TDF $b$ usando la separabilidad necesita en el primer paso $n_{c}^{2}$ multiplicaciones y $n_{c}^{2}-1$ sumas y en el segundo paso $n_{p c}^{2}$ multiplicaciones y $n_{p c}^{2}-1$ sumas. Eso hace un total de $n_{c}^{2}+n_{p c}^{2}$ multiplicaciones y $n_{c}^{2}+n_{p c}^{2}-2$ sumas para la TDF $b$ usando la separabilidad [7].

- Se puede obtener directamente la señal promediada en la primera etapa.

Independientemente del método del promediado, el valor clave para una primera aproximación más precisa a la señal de presión es el del número de ciclos a promediar. 


\subsubsection{Propuesta para definir el número de ciclos a promediar}

La revisión bibliográfica ( $[1,9-13])$ indica que no existe consenso en cuanto al número mínimo de ciclos necesarios a medir. La tabla (5.1) presenta un resumen de las propuestas de varios autores.

\begin{tabular}{|c|c|c|c|}
\hline Autor & $\mathrm{N}$ ciclos & Tipo del motor & Tipo de ensayos \\
\hline Armas [1] & 20 & 4 cilindros-diésel & Estacionarios \\
\hline Cartwright and Fleck [13] & 40 & $\begin{array}{l}2 \text { cilindros- } \\
\text { gasolina }\end{array}$ & Estacionarios \\
\hline Lancaster et al [9] & 40 & $\begin{array}{l}2 \text { cilindros- } \\
\text { gasolina }\end{array}$ & Estacionarios \\
\hline Macian [10] & 110 & $\begin{array}{l}2 \text { cilindros- } \\
\text { gasolina }\end{array}$ & Transitorios \\
\hline Randolph [11] & 300 & $\begin{array}{l}2 \text { cilindros- } \\
\text { gasolina }\end{array}$ & $\begin{array}{l}\text { Estudio de la deriva } \\
\text { térmica de los sensores }\end{array}$ \\
\hline Brunt and Emtage [12] & 300 & $\begin{array}{c}2 \text { cilindros- } \\
\text { gasolina }\end{array}$ & Transitorios \\
\hline Lancaster et al [9] & 300 & $\begin{array}{l}4 \text { y } 2 \text { cilindros- } \\
\text { gasolina }\end{array}$ & Transitorios \\
\hline
\end{tabular}

Tabla 5.1: Número de ciclos promediados en varios trabajos.

Tal variación suele ser justificada por las diferencias entre motores y en el mismo motor, por la diferente dispersión en puntos de funcionamiento distintos, e incluso entre diferentes zonas del ciclo [14].

En los motores de encendido provocado la dispersión cíclica es mucho más pronunciada. La mayor estabilidad de los procesos termofluidodinámicos de los motores diésel, hace que el número de ciclos a promediar sea menor.

El número de ciclos a promediar varía, por lo tanto, según el tipo de motor usado, el tipo de la cadena de adquisición y el procesado al que se va a someter la señal medida.

Para fines de diagnóstico de combustión y usando la cadena de adquisición de la presión en cámara expuesta anteriormente (Sensor piezoeléctrico y etapa de acondicionamiento con baja sensibilidad termodinámica, filtrado antialiasing, cadena de medida con 16 bits de resolución y una resolución angular de $0.5^{\circ}$ ), se ha planteado la metodología que se expone a continuación para determinar el número de ciclos optimo a promediar.

- En primer lugar, se define el punto de funcionamiento del motor donde la señal de presión en cámara representa mayor dispersión (caso más desfavorable) entre medidas consecutivas. Dicho punto viene definido principalmente por el régimen de giro y el grado de carga.

- Se toma un número elevado de ciclos de presión en cámara en ese punto. 
- Se estudia el error típico cometido en el promediado de $n_{c}$ ciclos consecutivos en cada ángulo de giro.

- Se establece una tolerancia máxima de dicho error y se toma por válido el número de ciclos a promediar que cumpla dicha tolerancia.

- Se comprueba la validez de dicho número de ciclos a promediar evaluando el error típico obtenido en la dFQL calculada a partir de la señal de presión promediada.

Para los motores diésel, dado que durante la combustión aparecen más oscilaciones que durante la compresión o el lazo de bombeo, para determinar el punto de funcionamiento del motor, donde la presión en cámara representa mayor dispersión entre medidas consecutivas, es necesario efectuar un análisis en función del régimen de giro y el grado de carga del motor.

Es necesario advertir que una mayor dispersión entre las medidas de presión para un mismo punto de funcionamiento no quiere decir, directamente, mayor grado de dispersión en las combustiones tal y como se demostrará a continuación. De hecho, varios autores consideran que el motor siempre es más inestable a bajo régimen y bajo grado de carga $[4,10]$. Dicha inestabilidad, que se refiere a mayor grado de combustiones desiguales y definida en ocasiones como la dispersión cíclica, viene cuantificada mediante la covarianza de la pmi calculada mediante la ecuación5.2.15:

$$
\operatorname{CoV}(p m i)_{i}=\frac{\sigma_{p m i_{i}}^{2}}{\overline{p m i_{i}}}=\frac{\left(\sqrt{\sum\left(\overline{p m i_{i}}-p m i_{i, k}\right)^{2}}\right)^{2}}{n_{c} \cdot \overline{p m i_{i}}}
$$

Siendo:

$$
\begin{array}{ll}
\sigma & \text { la desviación típica. } \\
\overline{p m i_{i}} & \begin{array}{l}
\text { el valor medio de la presión media indicada en el punto } i \text { de } \\
\text { funcionamiento del motor. }
\end{array} \\
p m i_{i, k} & \text { la presión media indicada en el punto } i \text { de cada ciclo } k \text { medido. }
\end{array}
$$

La figura (5.3) representa la distribución de la covarianza del pmi en función del punto de funcionamiento de un motor diésel (DW10). Se puede confirmar que, a bajo régimen y baja carga, la covarianza del pmi es muy elevada (2-3\%), sin embargo, en zonas de muy alto régimen y baja carga, reaparece este fenómeno con covarianzas de hasta el $5 \%$.

Si bien, los valores pueden variar en función del motor y los sistemas de adquisición de datos, la tendencia es muy repetitiva.

Entre las causas que provocan dicha tendencia, Lee y otros [15] demuestran que el efecto de la deriva térmica de los sensores sobre el error cometido en la medida de la presión en cámara, debido a las combustiones, es de suma importancia. Además, según los mismos autores, se puede relacionar el error cometido en la medida de la presión máxima con el error cometido en el cálculo de la presión media efectiva y, por lo tanto, con su covarianza. 


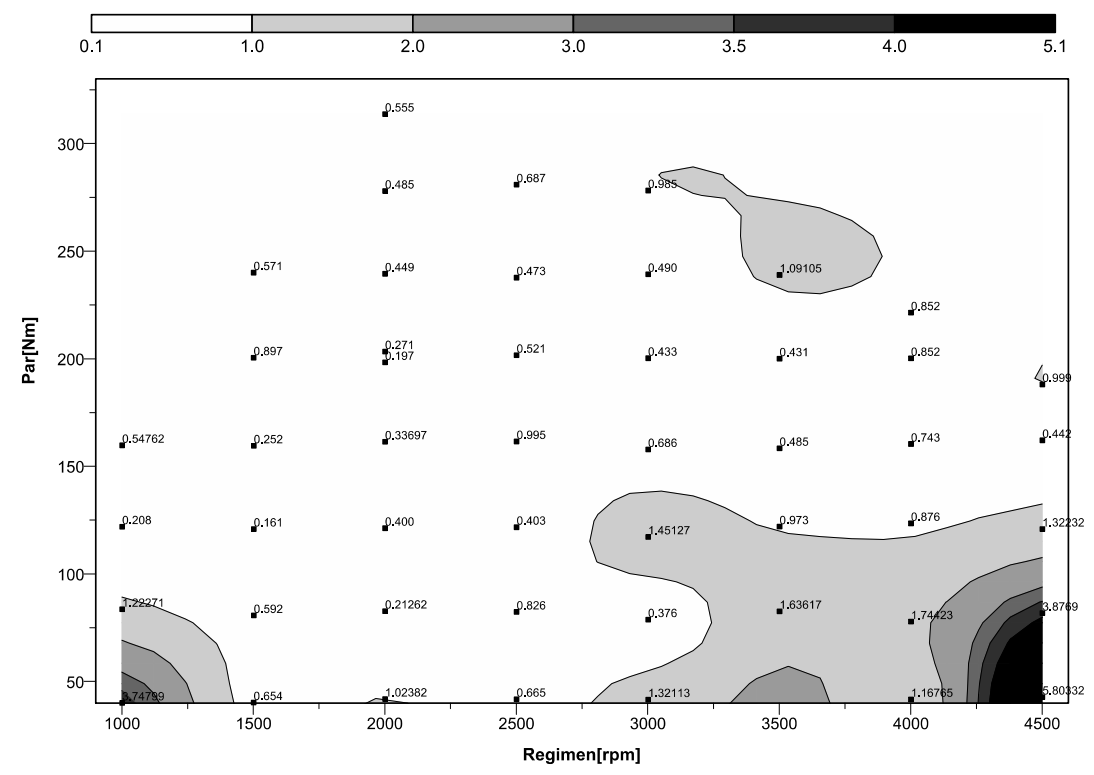

Figura 5.3: Distribución de la covarianza de la pmi en función del punto de funcionamiento de un motor diésel

Sin embargo, siendo el pmi un valor correspondiente a un ciclo termodinámico, es lógico esperar que, para un punto de funcionamiento estable, la acumulación de las variaciones reales y los errores de medida de la presión cometidos en cada ángulo de giro y principalmente en la zona de combustión, son la causa de la variación del pmi entre ciclos y, por lo tanto, en la covarianza del pmi aunque la variación máxima de la medida de presión tenga un peso significativo.

En cada punto de funcionamiento del motor de la figura (5.3) y a cada ángulo de giro del motor se calcula la covarianza de las presiones medidas para sucesivos ciclos según la fórmula:

$$
\operatorname{CoV}(p)_{i, \alpha}=\frac{\sigma_{p_{i, \alpha}}^{2}}{\overline{p_{i, \alpha}}}=\frac{\left(\sqrt{\sum\left(\overline{p_{i, \alpha}}-p_{i, \alpha, k}\right)^{2}}\right)^{2}}{n_{c} \cdot \overline{p_{i, \alpha}}}
$$

Donde:

$$
\begin{array}{ll}
\sigma & \text { desviación típica. } \\
n_{c} & \text { número de ciclos medidos en el punto de funcionamiento } i .
\end{array}
$$$$
\text { desviación típica. }
$$$$
\overline{p_{i, \alpha}} \quad \text { valor medio de la presión en el punto } i \text { de funcionamiento del }
$$
motor para el ángulo de giro del motor $\alpha$. 
$p_{i, \alpha, k} \quad$ presión medida en el punto $i$ de funcionamiento del motor, ángulo $\alpha$ y del ciclo $k$ medido.

La figura (5.4) representa la variación de la desviación típica de la presión en cámara en función del ángulo de giro. En dicha figura, se representa para cada ángulo de giro la desviación típica entre 50 medidas de presión en cámara, correspondientes a 50 ciclos en un punto de funcionamiento estable $(3000 \mathrm{rpm}, 84 \mathrm{Nm})$ del motor M9R. También se representa la tasa adimensional de liberación de calor obtenida a partir de la señal de presión promediada y filtrada. Se aprecia que, a partir de las inyecciones (presentadas por zona sombreada), y precisamente en presencia de la combustión, la desviación típica se ve incrementada notablemente. Dicha apreciación es repetitiva para distintos puntos de funcionamiento del motor (figura (5.5)).

Teniendo en cuenta este fenómeno, para definir el punto de funcionamiento del motor donde la señal de la presión en cámara representa mayor dispersión, bastaría con evaluar la desviación típica en función del ángulo para distintos puntos representativos del funcionamiento del motor. También se puede usar como criterio la varianza o la covarianza, aunque esta última puede complicar el análisis en ángulos de baja presión.

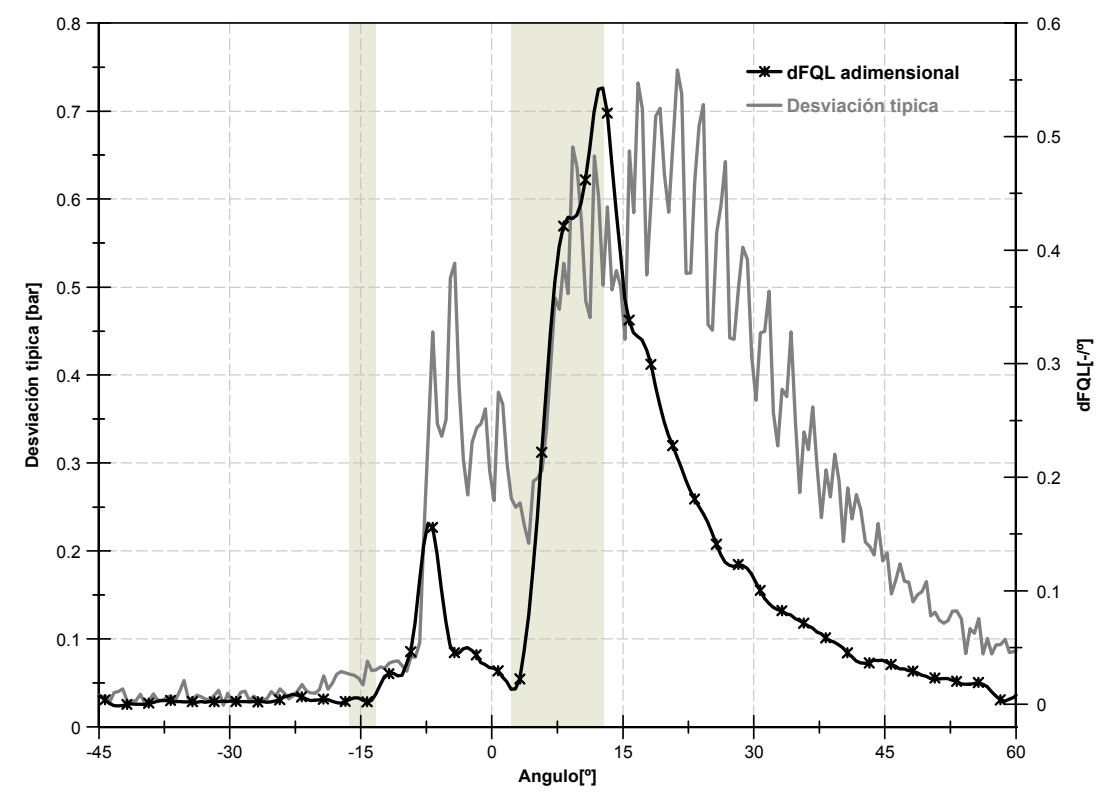

Figura 5.4: Desviación típica de la presión entre varios ciclos en función del ángulo de giro 


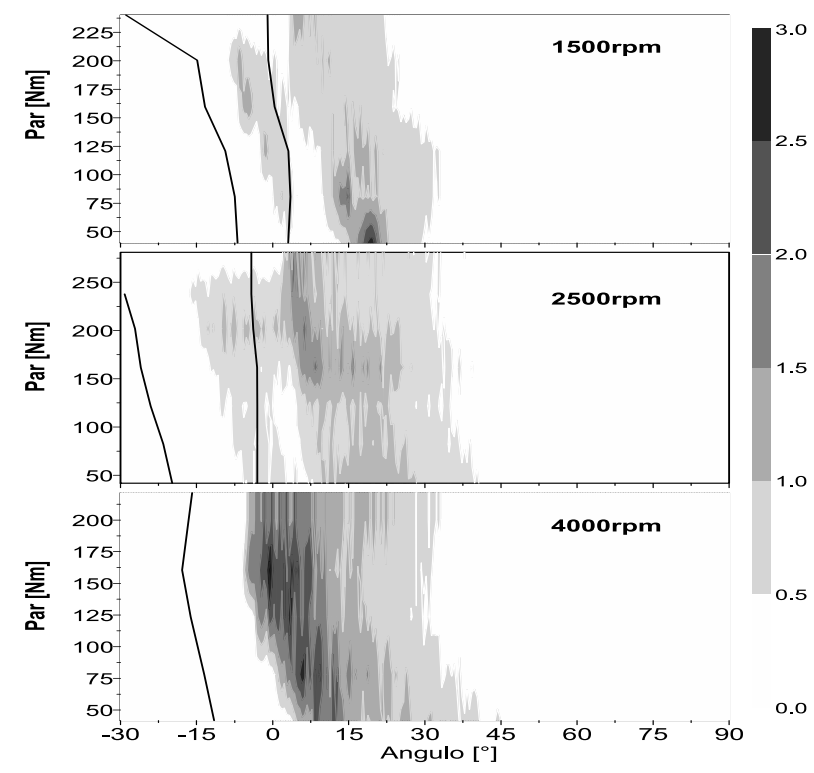

Figura 5.5: Desviación típica de la presión a varios regímenes y cargas.

La figura (5.6) representa la covarianza máxima de las presiones medidas. Dicha covarianza máxima, se obtiene a partir de los valores correspondientes a las covarianzas calculadas mediante la ecuación (5.2.16) en las zonas de combustión con el fin de evitar valores engañosos a bajas presiones medias. Se observa que las máximas covarianzas se localizan a baja carga y altos regímenes (valores que superan el $2.6 \%$ ).

Comparando la figura (5.3) y la figura (5.6) se puede apreciar que a baja carga y bajo régimen las combustiones son mucho menos estables y que a alto régimen el ruido presente en las medidas toma un peso significativo.

Dicha inestabilidad es debida a que a bajo régimen y baja carga, las bajas temperaturas, las tasas elevadas de EGR y las múltiples inyecciones afectan en la combustión y, por lo tanto, en la repetitividad de la presión en cámara, lo que se traduce en una mayor desviación típica entre las muestras tomadas para cada ángulo de giro. Sin embargo, a alto régimen y alta carga, aun en ausencia de EGR y de inyecciones múltiples, las altas masas de combustible inyectadas y las altas temperaturas provocan combustiones más intensas y de larga duración acompañadas con oscilaciones más significativas en la señal de presión, lo que se traduce en una mayor desviación típica de las medidas.

Además, al usar un muestreo angular, a mayor régimen de giro mayor frecuencia temporal de muestreo, por lo que, a mayor régimen de giro mayor muestras de señal de presión son tomadas durante la combustión y, por lo tanto, mayores serán las oscilaciones en la medida de la señal de presión en cámara.

Por este motivo, se considerará la zona de funcionamiento de alto régimen y alta carga, 


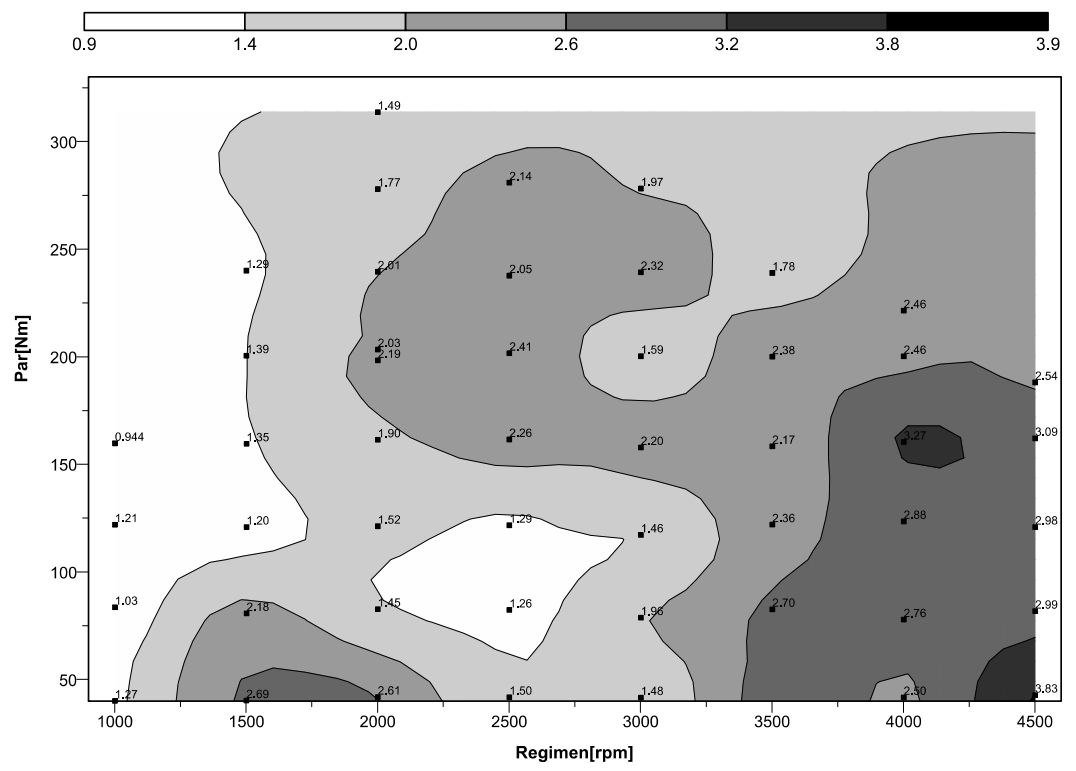

Figura 5.6: Distribución de la máxima covarianza de la presión en función del punto de funcionamiento de un motor diésel

como la zona donde se produce menor repetitividad en la medida de la señal de presión en cámara y, por lo tanto, la más adecuada para determinar el número de ciclos óptimo a promediar para obtener la señal de presión en cámara más precisa.

Como se comentó anteriormente, a medida que aumenta el número de muestras a promediar disminuye el error típico ya que la señal promediada converge a la media de un número infinito de ciclos. En la figura (5.7), se representa, para un punto de funcionamiento de motor con alto régimen y alto grado de carga, la desviación típica máxima, a lo largo de un ciclo, entre ciclos de presión promedios en función del número de ciclos promediados. Se aprecia que a partir de 20 ciclos dicha desviación es inferior a los 100 mbar, consiguiendo así un error típico inferior a los 20 mbar. En la misma figura, se aprecia que para una señal de presión en cámara obtenida a partir del promedio de 20 ciclos medidos, el error típico cometido en el cálculo de la $d F Q L$ es inferior a 0.1 $\mathrm{J} /{ }^{\circ}$, validando el criterio que considera 20 ciclos como un número adecuado de ciclos a promediar para análisis de la combustión en motores diésel. Para mayor precisión se usará el promedio de 25 ciclos en el desarrollo del presente trabajo. 

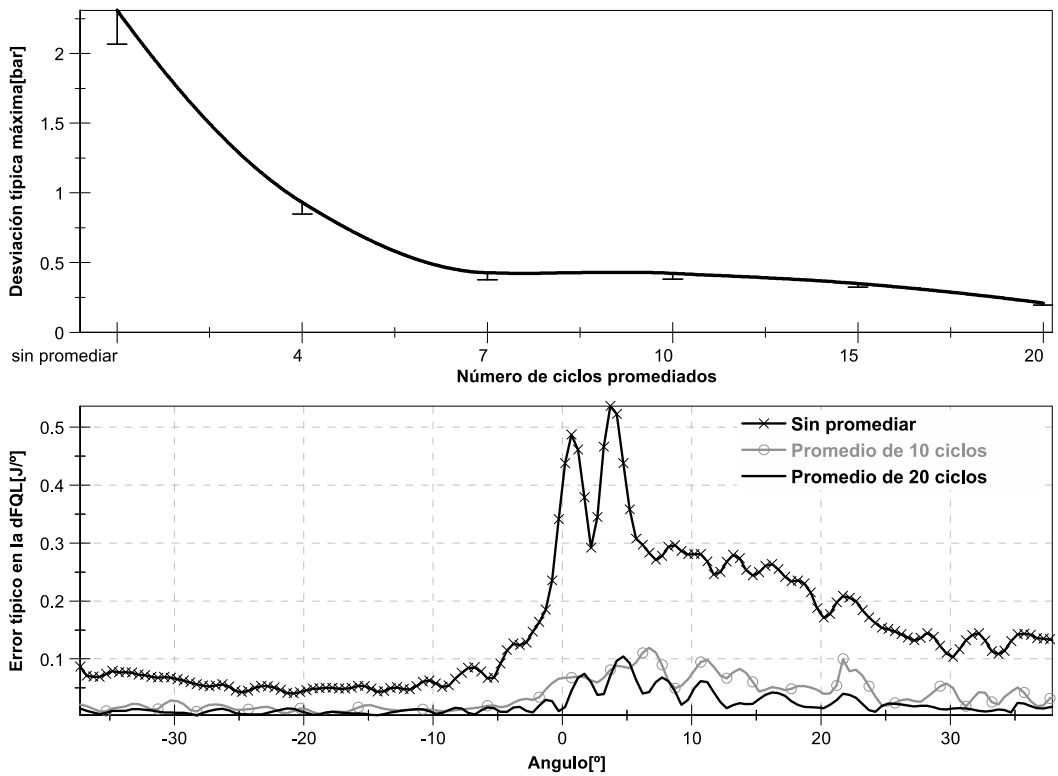

Figura 5.7: Efecto del número de ciclos de presión a promediar

\subsection{Etapa 2. El filtrado digital de la señal de presión}

Los filtros digitales son filtros estables y ampliamente empleados [8],[20]y [22]. Estos filtros tienen como ventajas principales una alta inmunidad al ruido, alta precisión y fácil implementación y modificación de sus características además de su bajo coste.

Se pueden dividir principalmente en dos categorías: filtros recursivos y filtros no recursivos tabla (5.2) .

\begin{tabular}{|c|c|}
\hline Forma matemática & Tipo y ejemplo \\
\hline$y_{i}=\sum_{k} h_{k} \cdot x_{i-k}$ & $\begin{array}{c}\text { No Recursivo (FIR): Media móvil (MA) } \\
\text { orden } k\end{array}$ \\
\hline$\sum_{k} g_{k} \cdot y_{i-k}=x_{i}$ & Recursivo (IIR) : Autoregresivo (AR) orden $k$ \\
\hline$\sum_{k_{1}} g_{k_{1}} \cdot y_{i-k_{1}}=\sum_{k_{2}} h_{k_{2}} \cdot x_{i-k_{2}}$ & $\begin{array}{l}\text { Recursivo (IIR), Autoregresivo y media en } \\
\text { movimiento }\left(\operatorname{ARMA}\left(k_{1}, k_{2}\right)\right)\end{array}$ \\
\hline
\end{tabular}

Tabla 5.2: Tipos de filtros digitales

Los filtros digitales recursivos también se denominan "de respuesta infinita al impulso" (Infinite Impulse Response IIR). Su respuesta es función de la excitación y también de las respuestas anteriores, ya que son filtros con realimentación donde la 
salida no depende sólo de la entrada actual " $x_{i}$ ", sino también de valores pasados de la salida " $y_{i}$ ". Presentan en general distorsión de fase puesto que la fase no es lineal con la frecuencia.

Los filtros digitales donde no haya recursividad, se denominan de "Respuesta finita al Impulso" (Finite Impulse Response FIR), ya que si la entrada se mantiene nula durante periodos consecutivos, la salida será también nula. La respuesta es, por lo tanto, de duración finita, siendo una suma ponderada de valores pasados y presentes de la entrada. De ahí que a algunos de estos filtros se les denomine de Media Móvil (Moving Average MA).

Los filtros FIR son de fase lineal y son siempre estables. Por lo tanto, se puede compensar el desfase y son los más convenientes para tratamiento de señales donde se requiere conocimiento y precisión en el desfase de la señal como es el caso de señales de presión en cámara.

El orden de un filtro IIR es mucho menor que el de un filtro FIR para una misma aplicación. Eso hace que los filtros IIR sean los más adecuados para filtrar señales en tiempo real.

Para determinar el orden y los coeficientes de los filtros digitales suele utilizarse el dominio de frecuencias, puesto que el objetivo final del filtrado es separar frecuencias consideradas ruido de las restantes que se pretenden estudiar. Es decir, estos filtros deben permitir que algunas señales pasen inalterables, mientras se realiza un bloqueo a las otras frecuencias. Para dichos filtros se definen las siguientes variables:

- La banda de paso que se refiere al rango de frecuencias a conservar.

- La banda de interrupción donde se sitúan aquellas frecuencias que son eliminadas.

- La banda de transición se encuentra entre las dos anteriores. Suele expresarse como una fracción de la frecuencia de muestreo, y se caracteriza por el grado de rapidez de extinción o de apagado (roll-off) que a medida que se incrementa la banda de transición se hace más estrecha.

El límite entre la banda de paso y la banda de transición es conocido como la frecuencia de corte. En el diseño de un filtro analógico, la frecuencia de corte se encuentra ubicada habitualmente donde la amplitud de una señal con dicha frecuencia, se reduce al $70.7 \%$. En los filtros digitales, su ubicación es menos estandarizada y es común encontrarse con el $99 \%, 90 \%, 70.7 \%$ y $50 \%$ de los niveles de amplitud definidos para la frecuencia de corte.

Cuando se sabe con exactitud las frecuencias indeseables en una señal, el filtro ideal sería aquel que anula la magnitud de los armónicos o frecuencias no deseadas y mantiene las restantes. Si se calcula la transformada de Fourier inversa de esta respuesta en frecuencia, se obtiene el núcleo del filtro ideal (kernel) que no es más que la función matemática "sinc" $(\sin (x) / x$ de la ecuación (5.2.6)). Esta función, es empleada por medio del teorema de convolución, mencionado anteriormente, que afirma que la convolución en el dominio temporal es equivalente al producto punto a punto en el dominio espectral o de frecuencias. 
En la práctica, la realización del filtro ideal no es posible puesto que la función "sinc" es definida en todo el intervalo más menos infinito, lo que requiere un número infinito de muestras en la señal. Para evitar este problema, se toma solamente una cantidad (orden del filtro) simétrica y finita de valores de la función "sinc" y se desplaza para que empiece desde el origen.

Esta aproximación puede presentar sobre-valores "overshoot" en la respuesta debidos al fenómeno denominado "Gibbs", que se manifiesta cuando la función que se está desarrollando en serie de Fourier tiene discontinuidades que se generan en los bordes de la función, lo que imposibilita una buena convergencia en las proximidades de las discontinuidades y afecta gravemente a las derivadas de las señales obtenidas.

En el uso de las señales de presión en cámara, el efecto anterior tiene graves consecuencias sobre la derivada de la presión o en cálculo de la $d F Q L$ [16].

Para suavizar este fenómeno se debe suavizar la banda de transición del filtro (aumentar el roll-off) usando filtros digitales FIR de tipo sinc-ventanado "windowedsinc" con banda de transición suavizada mediante la multiplicación de los coeficientes del filtro ideal aproximado con funciones, como la de Blackman, Hamming, Hanning, etc. entre otros [17-19]. Por lo tanto, se considerará el armónico o frecuencia de corte $(f c)$ y el ancho de la banda de transición como parámetros básicos de diseño de los filtros.

El ancho de banda de transición normalizada está directamente relacionado con el orden del filtro por medio de la ecuación siguiente:

$$
\text { Orden del filtro }=\frac{4}{\text { Ancho de la banda de transición }}
$$

Un ancho de banda de transición no nulo, permite suavizar el fenómeno de Gibbs, sin embargo, supone darle magnitud no nula a frecuencias "no deseadas" en la señal filtrada. Por lo tanto, hay un compromiso entre la rapidez de caída, de dicha magnitud y el tiempo de cálculo necesario para calcular la convolución y, en consecuencia, el tiempo de calculo de la señal filtrada.

El tiempo de cálculo de la señal filtrada no supone ningún problema para el tratamiento offline de la señal, no obstante, en el tratamiento en tiempo real, hay que considerar seriamente el orden del filtro a aplicar.

Para el filtrado de la presión en cámara, cuando la frecuencia de muestreo es variable en función del régimen de giro, la elección de los parámetros del filtro no es trivial. Por lo tanto, se debe plantear el diseño en función de la cantidad de frecuencias existentes en el punto de ensayo (arrastre o combustión) y el régimen de giro (frecuencia de muestreo).

En la figura (5.2) se aprecia que el espectro del ciclo medio puede dividirse en tres zonas:

- Zona de bajas frecuencias que corresponde a variaciones lentas de la presión debida al trabajo mecánico y la transmisión de calor.

- Zona de medias frecuencias que solo aparecen en combustión

- Zona de altas frecuencias debida básicamente a fenómenos de resonancia (de combustión y del sensor) y ruido. 
Al estudiar la combustión, la zona de altas frecuencias debe ser eliminada sin alterar la zona de medias frecuencias. Definir el final de cada zona es, por tanto, crucial a la hora de diseñar los filtros digitales de la señal de presión en cámara.

Además, al hacer un muestreo angular, la frecuencia de muestreo temporal es variable, por lo tanto, a medida que aumente el régimen de giro del motor, la señal muestreada contendrá frecuencias más altas. Siendo la relación entre el armónico de corte " $k_{c}$ " y la frecuencia temporal de corte " $f_{c}$ ":

$$
f_{c}[\mathrm{~Hz}]=k_{c} \cdot \frac{n[\mathrm{rpm}] \cdot \frac{360[\mathrm{o} / \mathrm{rev}]}{60[\mathrm{~s} / \mathrm{min}]}}{f_{\text {m, angular }} \cdot n p c}
$$

Donde:

$$
\begin{array}{ll}
f_{m, \text { angular }} & \text { es la frecuencia de muestreo angular }\left(0.5^{\circ}\right) \text { que aporta } n p c \\
\text { muestras por ciclo(1440). }
\end{array}
$$

\subsubsection{Métodos experimentales para determinar los parámetros del filtro}

Los métodos experimentales usados para determinar los parámetros de filtros digitales, se han elaborado tomando como criterio la calidad de la curva de calor transmitido a las paredes, calculado a partir de la señal de presión, tal como propone Lapuerta y otros [20], multiplicado por el exponente politrópico muy sensible al filtro, ya que emplea la derivada de la presión para el cálculo.

Los mejores resultados se obtuvieron para los valores de armónico de corte $k_{c}$ y ancho de banda de transición mostrados en la tabla (5.3).

\begin{tabular}{|l|c|c|c|c|}
\cline { 2 - 5 } & Arrastre & \multicolumn{3}{|c|}{ Combustión } \\
\hline \hline $\begin{array}{l}\text { Régimen de giro } \\
(\boldsymbol{n}[\mathbf{r p m}])\end{array}$ & 55 & $n<1250$ & $1250<n<4000$ & $n>4000$ \\
\hline Armónico de corte $k_{c}$ & 60 & \multicolumn{3}{|c|}{$k_{c}+30$} \\
\hline $\begin{array}{l}\text { Fin de la banda de } \\
\text { transición } k_{\text {stop }}\end{array}$ & & \multicolumn{3}{|c}{} \\
\hline
\end{tabular}

Tabla 5.3: Parámetros del filtro digital.

Siendo el filtro FIR usado de tipo sinc-ventanado con banda de transición suavizada mediante función de Hanning [21]:

$$
\left\{\begin{array}{l}
\theta_{k}=1 \\
\theta_{k}=\frac{1}{2} \cdot\left[\cos \left(\frac{k-\left(k_{c}-\frac{B W}{2}\right)}{B W} \cdot \pi\right)+1\right] \\
\theta_{k}=0
\end{array}\right.
$$


Siendo $k_{c}$ el armónico de corte cuya magnitud se reduce al $50 \%$ mediante el filtro $\left(\theta_{k_{c}}=0.5\right)$ y el ancho de banda $B W=2 \cdot\left(k_{s t o p}-k_{c}\right)$.

El filtro propuesto ha sido probado en varios motores y distintos tipos de sensores arrojando resultados muy satisfactorios. No obstante, Armas y Martín [1,4] consideran el filtro demasiado conservador y aconsejan visualizar en cada caso el espectro previamente a su filtrado con el objetivo de detectar posibles irregularidades y garantizar así la idoneidad del filtro propuesto.

\subsubsection{Propuesta para determinar los parámetros del filtro}

El método anterior para determinar los parámetros del filtro puede presentar limitaciones al aplicar puntos de corte fijos (solo dependientes del régimen de giro), ya que, para los ensayos en combustión, las condiciones de funcionamiento difieren mucho entre un punto y otro y, por tanto, el proceso de combustión dentro del cilindro es muy diferente y, en consecuencia, la presión medida también lo es. Para evitar trabajo costoso de análisis de la frecuencia de corte, se propone elegir el armónico de corte a partir de la información contenida en el propio espectro de una medida de varios ciclos consecutivos y teniendo en cuenta, además del régimen de giro, la carga del motor.

En caso de arrastre, el armónico de corte obtenido, mediante el criterio de la calidad de la curva de calor por el exponente politrópico utilizado en los métodos experimentales, suele coincidir con aquél armónico donde converge el armónico del ciclo medio a los armónicos restantes, es decir, el inicio de la zona del espectro donde no se puede diferenciar el armónico del ciclo medio de los demás armónicos.

En la figura (5.8) se ilustra la diferencia de la amplitud entre el armónico del ciclo medio y la de la media de los $n_{c}-1$ armónicos siguientes (los que no corresponden al ciclo medio como se explicó antes 5.2). Dicha diferencia se calcula a partir del espectro de la medida de la señal de presión de tamaño $N\left(n_{c}\right.$ ciclos $)$ mediante la fórmula:

$$
\operatorname{dif}(k)=\text { Amplitudarmónico }\left(k \cdot n_{c}\right)-\frac{\sum_{i=1}^{n_{c}-1} \text { Amplitudarmónico }\left(k \cdot n_{c}+i\right)}{n_{c}-1}
$$

Con $k \in\left[1, \frac{N}{2}\right]$

Del mismo modo, para obtener el armónico de corte en caso de combustiones, se estima el armónico de corte como: el armónico donde la media de los armónicos que no corresponden al ciclo medio es casi igual al armónico del ciclo medio, es decir, el armónico $k$ donde la $\operatorname{dif}(k)$ de la 5.3.1 se mantiene en valores mínimos.

Desarrollando esta metodología para varios motores y en diferentes puntos de funcionamiento, se han obtenido los resultados ilustrados en la figura (5.9) para varios regímenes de giro y distintos grados de carga. Definiendo el armónico de corte en el punto donde la diferencia de la ecuación (5.3.1) es inferior a $0.25 \%$ de la máxima diferencia de cada ensayo. Además, se ha definido el armónico del fin de la banda de transición como el armónico, siguiente al armónico de corte, cuya amplitud supere el $0.5 \%$ de la máxima diferencia. De esta forma se asegura que ningún armónico posterior al del de corte, tendrá 


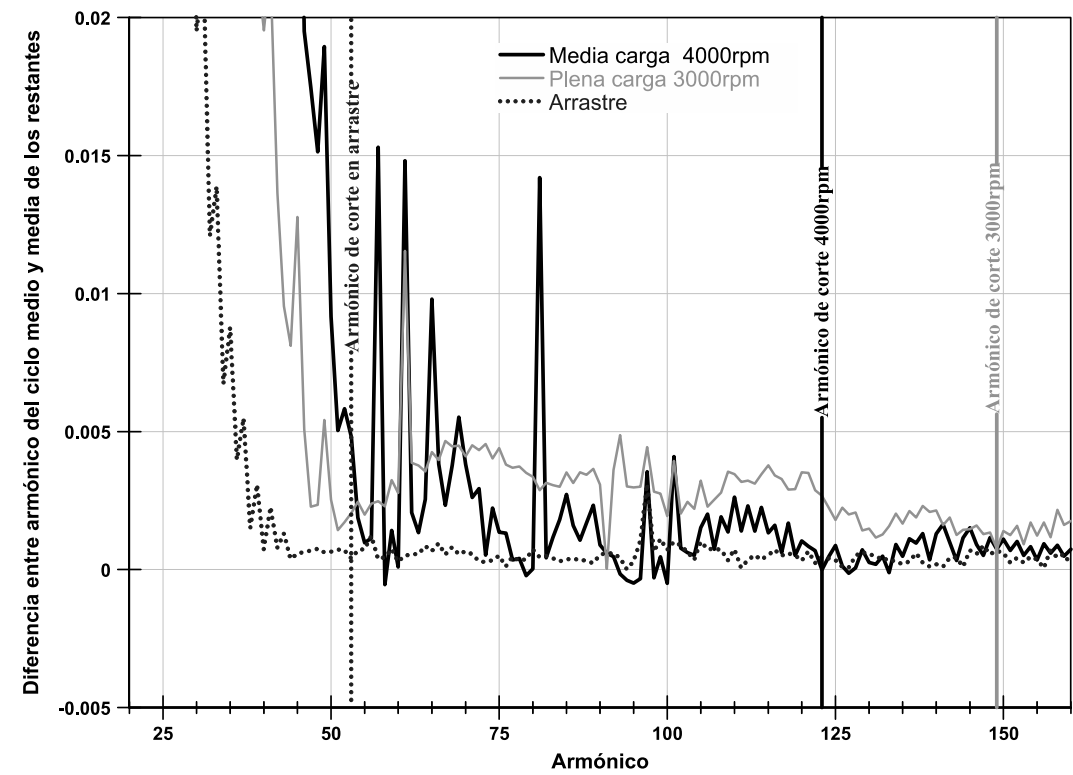

Figura 5.8: Criterio de selección de la frecuencia de corte

una amplitud superior a la del armónico de corte. Estas tolerancias, deben ser ajustadas en caso de sistemas de medida menos precisos.

La presente metodología aporta resultados muy satisfactorios al filtrar señales promediadas de 25 ciclos consecutivos, dando como resultado una desviación típica entre $d F Q L$ calculadas inferior a $0.1 \mathrm{~J} /{ }^{\circ}$ para todos los puntos de funcionamiento de los motores probados. Además, los valores de las frecuencias de corte son muy cercanos a plena carga a los obtenidos por métodos tradicionales (tabla (5.3)).

En esta alternativa no se toma en cuenta la dispersión de los armónicos. Para ello, se puede completar la metodología propuesta por medio del uso de herramientas estadísticas, determinando el armónico de corte como aquél donde existe una probabilidad superior al $1 \%$ de que la diferencia entre la distribución de armónicos del ciclo medio y la de los armónicos restantes sea negativa, es decir, cuando el intervalo de confianza de dicha diferencia adquiera valores negativos [22]. Para el armónico del final de la banda de transición, esta probabilidad se incrementa al $10 \%$.

El método propuesto muestra una gran repetitividad, tanto en condiciones de arrastre como en combustión, cuando se aplica a un número diferente de ciclos consecutivos de la medida de presión en cámara, observándose que la obtención del primer punto de corte converge al mismo valor a medida que el número de ciclos aumenta. La utilización del filtro ha demostrado ser valiosa en su aplicación para la medida de la presión en cámara de MCIA y, en cuanto la derivada de la misma (utilizada para el cálculo de $d F Q L$ ), es 


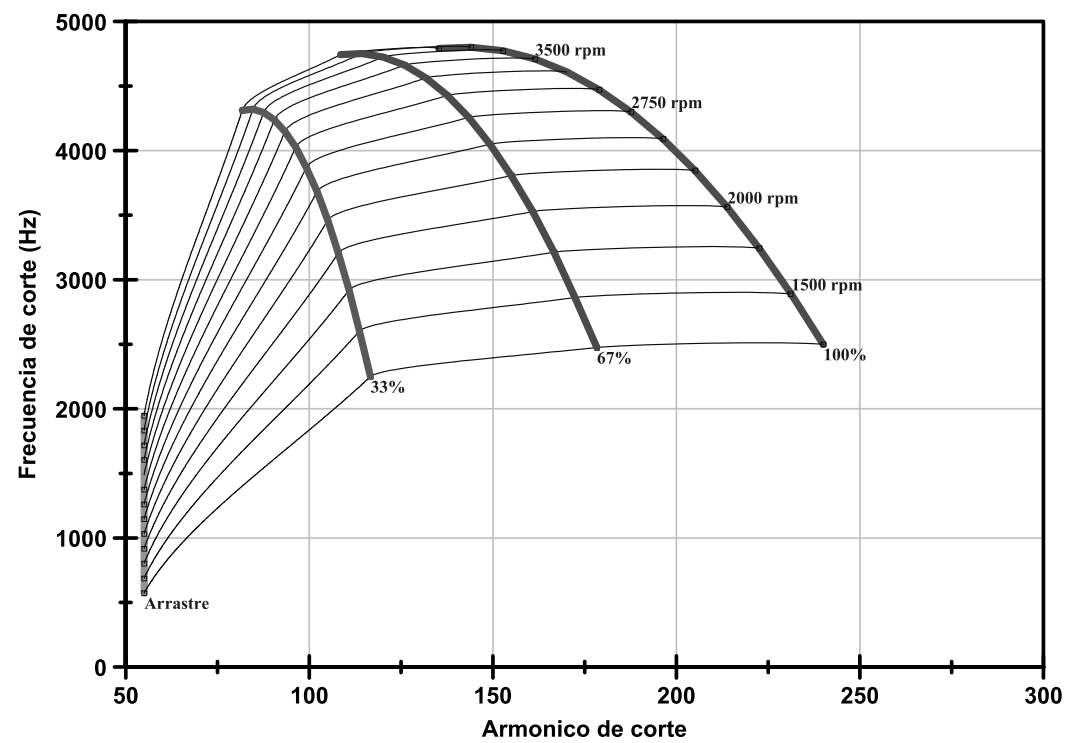

Figura 5.9: Parámetros del filtro por Método alternativo

suavizada $[23,24]$.

De todas formas, como se aprecia en la figura (5.9), los parámetros de los filtros obtenidos satisfacen dos criterios de suma importancia:

- En primer lugar, se rechazan componentes de la señal con frecuencias mayores a $6 \mathrm{kHz}\left(k_{c}<6 \mathrm{kHz}\right)$, que generalmente no están relacionados con el proceso de la combustión [25, 26].

- En segundo lugar, se atribuyen a puntos de funcionamiento de baja carga (con mayor combustiones inestables) bandas de transición menos estrechas, permitiendo así menor oscilaciones en la respuesta de los filtros.

El mejor criterio de evaluación del filtro, resulta finalmente ser, la baja desviación de la $d F Q L$ obtenida. 


\subsubsection{Propuesta para el filtrado de la señal de presión en cilindro en tiempo real.}

Cuando se requiere el tratamiento online de la señal de presión en cámara, el promediado y el filtrado anteriormente mencionado no son viables, ya que se requiere un tamaño de memoria de datos importante y no es viable esperar 25 ciclos para disponer de una señal representativa. Por lo tanto, los criterios de diseño del filtro para aplicaciones en tiempo real y/o de control cambian significativamente. Sin embargo, los filtros diseñados para el tratamiento offline sirven de punto de partida y herramienta de validación de las prestaciones de los filtros en tiempo real.

Los filtros digitales en tiempo real deben tener el mínimo orden posible, además de tener la respuesta que mejor se ajuste al filtro ideal.

Para ello, se define la respuesta deseada del filtro FIR de paso bajo en todo el rango de funcionamiento de los motores diésel, basándose en las frecuencias de corte de los datos de la figura (5.9) y añadiendo las siguientes restricciones:

1. Un rizado $\delta_{p}$ en la ganancia de las amplitudes de la banda de paso inferior a $0.001 \mathrm{~dB}$

2. Una ganancia $\delta_{a}$ máxima de $-60 \mathrm{~dB}$ en la banda de paro.

3. Un ancho de banda de transición máximo de $1 \mathrm{kHz}$.

A partir de esta definición, se calculan los parámetros $h(i)$ del filtro cuyo espectro se ajuste mejor a dicha definición. La mejor forma de lograr este objetivo es usar el método de Remez [27], que sugiere minimizar el error máximo entre el filtro definido y el filtro ideal mediante algoritmos iterativos [19]. Dicho error viene definido por:

$$
|E(w)|=|D(w)-H(w)| \leqq \begin{cases}\delta_{p} & \text { para } 0<w=2 \cdot \pi \cdot \frac{f}{f_{m}}<2 \cdot \pi \cdot \frac{f_{c}}{f_{m}} \\ \delta_{a} & \text { para } 2 \cdot \pi \cdot \frac{f_{\text {stop }}}{f_{m}}<w<\pi\end{cases}
$$

Donde:

El filtro ideal viene representado por: $D(w)= \begin{cases}1 & \text { para } 0<w=2 \cdot \pi \cdot \frac{f}{f_{m}}<2 \cdot \pi \cdot \frac{f_{c}}{f_{m}} \\ 0 & \text { para } 2 \cdot \pi \cdot \frac{f_{\text {stop }}}{f_{m}}<w<\pi\end{cases}$

y $H(w)$ representa la TDF de los coeficientes del filtro, de orden $N$, a diseñar que cumplen con la ecuación:

$$
\begin{cases}h_{0}\left(i_{0}\right)=h_{0}\left(-i_{0}\right)=\frac{1}{N}\left[h(0)+\sum_{k=1}^{N / 2} 2 \cdot h\left(\frac{2 \pi k}{N}\right) \cdot \cos \left(\frac{2 \pi k}{N} \cdot i_{0}\right)\right] & \operatorname{con} i_{0}=0,1, \cdots, \frac{N}{2} \\ h(i)=h_{0}\left(i_{0}-\frac{N}{2}\right) & \operatorname{con} i=0,1, . . N-1\end{cases}
$$

Dicho método de diseño aporta los coeficientes de los filtros óptimos para tratar la señal de la presión en cámara en tiempo real presentados en figura (5.10).

Los parámetros correspondientes al filtro han sido programados en una plantilla de control en tiempo real aportando resultados ampliamente satisfactorios. Un ejemplo de ello se ilustra en la figura (5.11). En dicha figura se observa la reducción de la desviación 


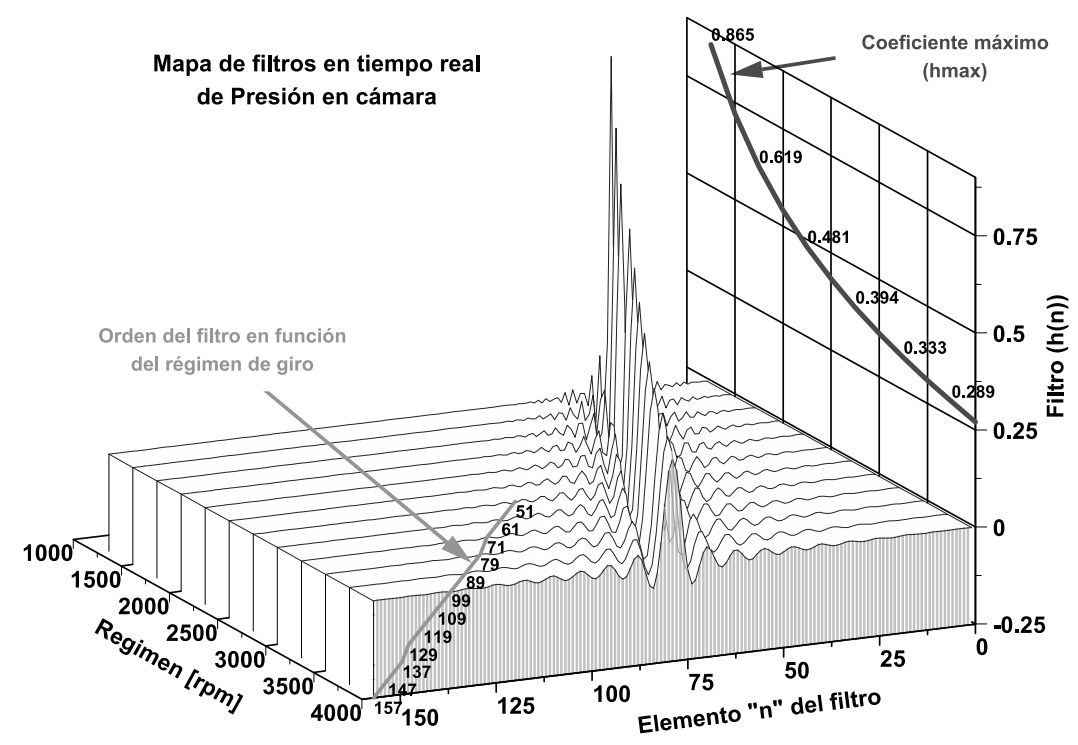

Figura 5.10: Parámetros del filtro en tiempo real para estudios de la combustión

típica, para el mismo ángulo de giro, entre ciclos consecutivos en un punto estable de funcionamiento del motor (DW10 a $2500 \mathrm{rpm}$ y plena carga). Desviación que se asemeja razonablemente a la obtenida por la aplicación de los filtros offline.

\subsection{Propuesta para la medida de la derivada de la presión en cámara}

Dada la complejidad y el alto coste de los amplificadores de carga discutidos en el capítulo 3, se plantea para la medición de la derivada de la presión en cámara remplazar el amplificador de carga por una etapa electrónica que cumple el papel de seguidor de corrientes cuya salida será proporcional a la corriente proporcionada por el sensor (??).

La figura (5.12) representa el esquema del circuito seguidor de corrientes cuya salida cumple con la ecuación:

$$
U_{a}=R . i=R \cdot \frac{d q}{d t}=c t e \cdot \frac{d p}{d t}
$$

El bajo coste y la obtención directa de la derivada de la señal de presión, muy fiel a los gradientes de presión que ocurren dentro del cilindro, son las ventajas principales de dicha alternativa. Sin embargo, se han observado las siguientes limitaciones:

- Para obtener la señal de presión se requiere integrar la señal, y al ser la carga una 


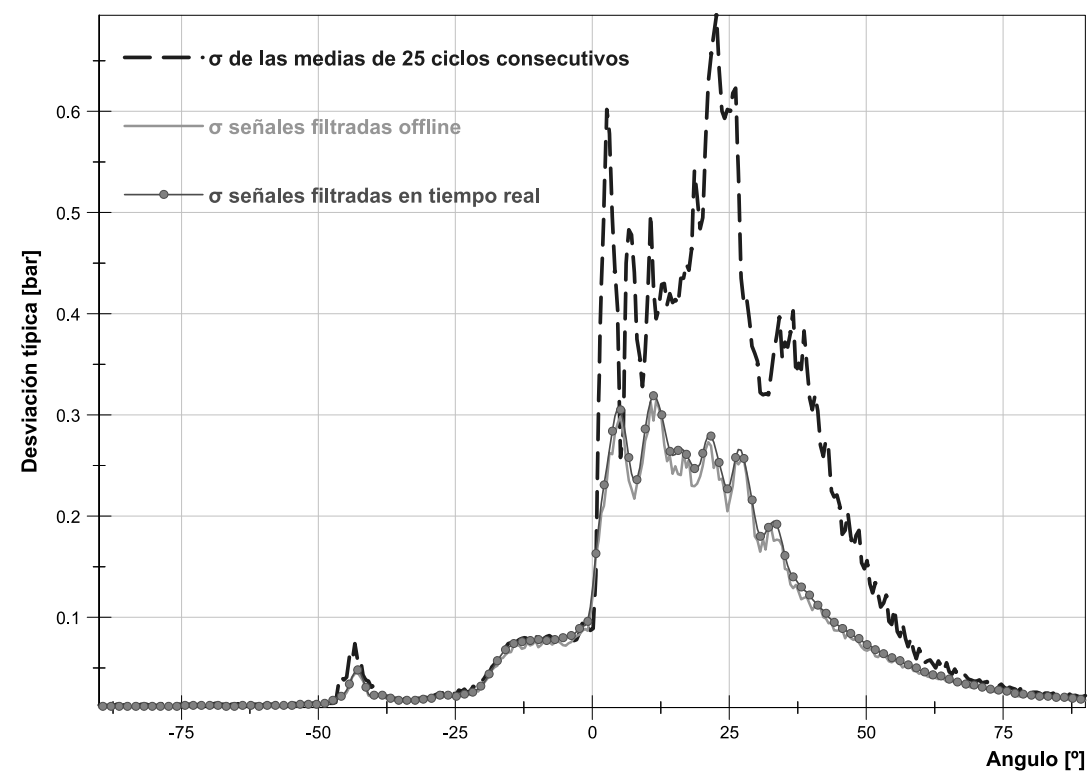

Figura 5.11: Prestaciones del filtro en tiempo real, para estudios de la combustión. (Motor DW10 a 2500 rpm y plena carga)

función temporal, cuando se muestrea en ángulo de giro de motor, se complica la integración numérica debido al tiempo variable entre muestras. Además, se requiere una frecuencia de muestreo muy alta.

- No se eliminan las cargas residuales del sensor, lo que se traduce en una posible saturación del sensor a largo plazo, además de la presencia de una deriva muy significativa cuando se integra la señal medida para obtener la presión.

- Para eliminar el efecto de las cargas residuales se ha incorporado una etapa electrónica que descarga el sensor al final de cada ciclo, aun así, la necesidad de altas frecuencias de muestreo temporal limitan la calidad de las presiones calculadas con esta alternativa.

La figura (5.13) muestra un ensayo real sobre motor policilíndrico a $2000 \mathrm{rpm}$ y plena carga, donde la señal de la presión en cámara ha sido medida mediante un sensor piezoeléctrico y un amplificador de carga y la derivada de la presión ha sido medida mediante el mismo sensor y un seguidor de corrientes. Las limitaciones comentadas anteriormente se ilustran en dicha figura donde se aprecia que la presión calculada a partir de la $d p$ medida presenta una cierta distorsión y una deriva importante debidas principalmente a la baja frecuencia que se ha empleado, a propósito, para dicho ensayo (una muestra cada $1^{\circ}$ ). 


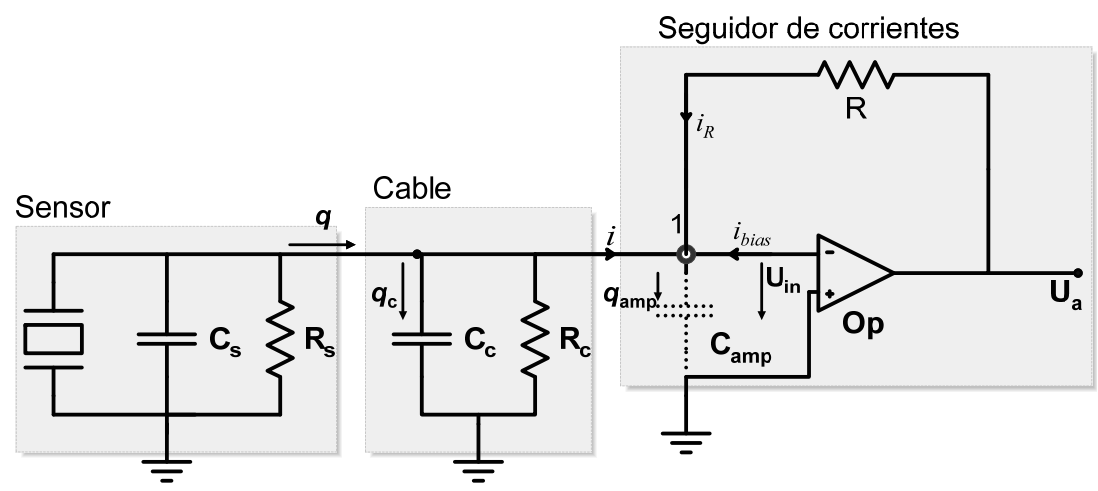

Figura 5.12: Seguidor de corrientes

En definitiva, si se requiere obtener la señal de presión en sí misma, este método no es recomendable, ya que sería más efectivo realizar una integración de forma analógica (frecuencia de muestreo infinita) usando los amplificadores de carga presentados anteriormente.

Sin embargo, para estudios basados en la derivada de la presión en cámara, la información aportada por esta alternativa es de suma importancia y será utilizada en fases posteriores de la presente tesis. De hecho, aunque la derivada de la señal medida directamente puede ser más beneficiosa, para validar las metodologías de diagnóstico de la combustión a partir de la derivada de la señal de presión en cámara, que se propondrán en el capítulo siguiente, el cálculo de la $d F Q L$ requiere la señal de presión en cámara. Sin embargo, es imposible registrar instantáneamente las dos señales (presión y su derivada), puesto que el mismo sensor no puede conectarse a dos etapas de acondicionamiento. Por lo tanto, se usarán por igual las señales medidas como las derivadas calculadas a partir de la señal de presión en cámara obtenida con el amplificador de carga para desarrollo de las metodologías. 

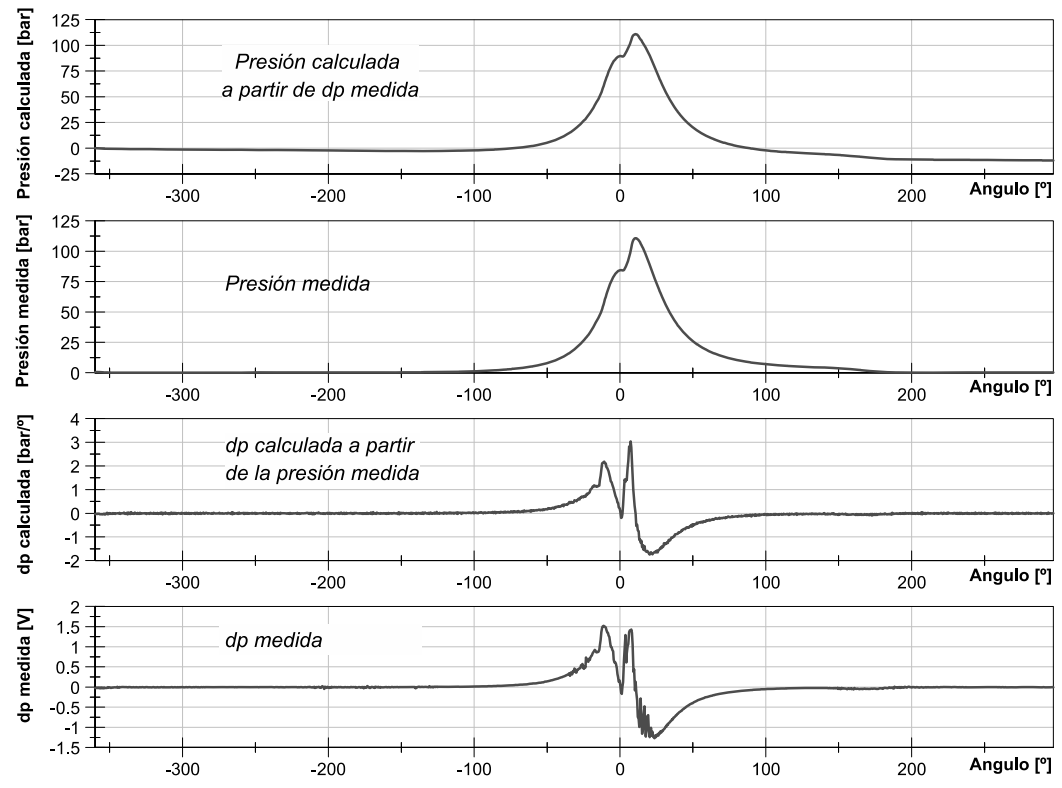

Figura 5.13: Comparativa entre medidas y cálculos obtenidos mediante amplificador de carga (presión) y seguidor de corriente(dp) 


\section{Bibliografía del capítulo}

[1] O. Armas. Diagnostico experimental del proceso de combustión en motores Diesel de inyección directa. Tesis Doctoral, Universidad Politécnica de Valencia, Valencia, Spain, 1998.

[2] M. Lapuerta, O. Armas, y J.J. Hernández. "Diagnosis of di diesel combustion from in-cylinder pressure signal by estimation of mean thermodynamic properties of the gas". Applied Thermal Engineering, 19:513-529, 1999.

[3] M. Lapuerta, O. Armas, y V. Bermúdez. "Sensitivity of diesel engine thermodynamic cycle calculation to measurement errors and estimated parameters". Applied Thermal Engineering, 20:843861, 2000.

[4] J. Martín. Aportación al diagnóstico de la combustión en motores Diesel de inyección directa. Tesis Doctoral, Universidad Politécnica de Valencia, 2007.

[5] P.R. Bevington y D. Robinson. Data Reduction and Error Analysis for the Physical Sciences. McGraw-Hill, 3rd edition, 2002.

[6] R. Bracewell. The Fourier Transform \& Its Applications. McGraw-Hill Science/Engineering/Math, 3rd edition, 1999.

[7] J.W. Cooley, P.A.W. Lewis, y P.D. Welch. "The fast fourier transform and its applications". Education, IEEE Transactions on, 12(1):27 -34, march 1969.

[8] R.C. González y R.E. Woods. Tratamiento digital de imágenes. Addison Wesley Iberoamericana, 1996.

[9] D.R. Lancaster, R.B. Krieger, y J.H. Lienesch. "Measurements and analysis of engine pressure data". SAE paper, (750026), 1975.

[10] V. Macian. Contribución al estudio de la influencia del movimiento del ruido sobre la dispersión cíclica y su caracterización. Tesis Doctoral, Universidad Politécnica de Valencia, 1984. 
[11] A.L. Randolph. "Methods of processing cylinder-pressure transducer signals to maximize data accuracy". SAE paper, (900170), 1990.

[12] M.F.J. Brunt y A.L. Emtage. "Evaluation of imep routines and analysis errors". SAE paper, (960609), 1996.

[13] A. Cartwight y R. Fleck. "Cylinder pressure analysis in high performance two stroke engines”. SAE paper, (962535), 1996.

[14] E. Oliver. Estudio de sistemas de adquisición y tratamiento de datos en motores de combustión interna alternativos. Tesis Doctoral, Universidad Politécnica de Valencia, Valencia, 1991.

[15] S. Lee, C. Bae, R. Prucka, G. Fernandes, Z.S. Filipi, y D.N. Assanis. "Quantification of thermal shock in a piezoelectric pressure transducer". SAE paper, 114, No. 3(2005-01-2092):1370-1381, 2005.

[16] S.X. Shi y H.Z. Sheng. "Numerical simulation and digital signal processing in measurements of cylinder pressure of internal combustion engines". IMechE, C20/87:211-218, 1987.

[17] L. Cohen. Time-Frequency Analysis. Prentice Hall Signal Processing Series. Prentice Hall PTR, 1995.

[18] B. Boashash. Time-Frequency Signal Analysis and Processing: A Comprehensive Reference. Elsevier, 2003.

[19] A. Antoniou. Digital Signal Processing: Signals, Systems, and Filters. McGraw-Hill Professional, Octubre 2005.

[20] M. Lapuerta, O. Armas, y S. Molina. "Study of the compression cycle of a reciprocating engine through the polytropic coefficient". Applied Thermal Engineering, 23:313-323, 2003.

[21] F.J. Harris. "On the use of windows for harmonic analysis with the discrete fourier transform". Proceedings of the IEEE, 66:51-83, 1978.

[22] E.L. Kosarev y E. Pantos. “Optimal smoothing of'noisy'data by fast fourier transform”. Journal of Physics E: Scientific Instruments, 16(6):537, 1983.

[23] F. Payri, J.M. Lujan, J. Martin, y A. Abbad. "Digital signal processing of in-cylinder pressure for combustion diagnosis of internal combustion engines". Mechanical Systems and Signal Processing, 24(6):1767 - 1784, 2010.

[24] F. Payri, P. Olmeda, C. Guardiola, y J. Martín. "Adaptive determination of cutoff frequencies for filtering the in-cylinder pressure in diesel engines combustion analysis". Applied Thermal Engineering, In Press, Corrected Proof:-, 2011. 
[25] J.M. Desantes, A.J. Torregrosa, y A. Broatch. "Wavelet transform applied to combustion noise analysis in high-speed di diesel engines". SAE paper, (2001-011545), 2001.

[26] F. Payri, A. Broatch, B. Tormos, y V. Marant. "New methodology for in-cylinder pressure analysis in direct injection diesel engines application to combustion noise". Measurement Science and Technology, 16:540-547, 2005.

[27] E. Ya. Remez. "Sur la détermination des polynômes d'approximation de degré donnée.". Comm. Soc. Math. Kharkov, 10:41, 1934. 

Capítulo 6

\section{Propuestas de métodos de parametrización de la combustión en tiempo real}




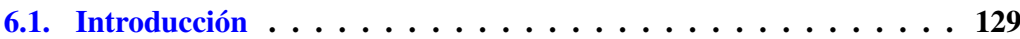

6.2. Síntesis y planteamiento de las metodologías . . . . . . . . 130

6.3. Metodología de diagnóstico de la combustión a partir del análisis temporal de la $d p . \ldots \ldots \ldots \ldots \ldots \ldots$

6.3.1. Herramientas de análisis de la señal en dominio temporal . . . 133

6.3.2. Aplicación del método basado en el análisis temporal de la derivada de la presión . . . . . . . . . . . . . . 136

6.3.3. Validación, análisis comparativo detección del SoC . . . . . 140

6.3.3.1. Caso de puntos de funcionamiento del motor con una inyección. . . . . . . . . . . . . . 140

6.3.3.2. Caso de puntos de funcionamiento del motor con varias inyecciones. . . . . . . . . . . . . 141

6.3.4. Validación, análisis comparativo detección del EoC . . . . . 150

6.3.5. Validación, análisis comparativo detección del ángulo de máxima $d F Q L \ldots \ldots$. . . . . . . . . . . . . . 153

6.3.6. Implementación en tiempo real. . . . . . . . . . . . . . 157

6.4. Metodología de diagnóstico de la combustión a partir del análisis en dominio de frecuencias de la $d p \ldots \ldots \ldots 16 \ldots$

6.4.1. Herramientas de análisis conjuntas de la señal en tiempo y frecuencia . . . . . . . . . . . . . 161

6.4.2. Aplicación de los métodos basados en el análisis en frecuencias de la $d p . \ldots \ldots$. . . . . . . . . . . . . . . 163

6.4.3. Aplicación de los métodos basados en el análisis conjunto Tiempo-Frecuencias de la $d p . \quad \ldots \ldots$

6.5. Análisis comparativo de las metodologías propuestas y conclusiones.173 Bibliografía del capítulo . . . . . . . . . . . . . . 176 


\subsection{Introducción}

Las relaciones de causa-efecto entre las fases de combustión, por una parte, los elementos encerrados en el cilindro, el trabajo producido y los contaminantes, por otra parte, están ampliamente estudiados y documentados. Principalmente la formación de los contaminantes ha sido objeto de numerosos estudios en las dos últimas décadas.

De hecho, las emisiones se forman como consecuencia del uso de una mezcla heterogénea de aire y combustible para la combustión. La variación en la relación airecombustible en la cámara de combustión provoca una variación drástica en la temperatura de combustión a través del cilindro que conduce a la formación de las emisiones no deseadas. Efectivamente, la presencia de zonas ricas en combustible genera mayor cantidad de monóxidos de carbón (CO) e hidrocarburos (HC) sin quemar y carbonilla u hollín, mientras que en las zonas pobres se produce una mayor cantidad de óxidos de nitrógeno (NOx), además de producirse oxidación de parte de los $\mathrm{CO}, \mathrm{HC}$ y carbonilla formados en las zonas ricas.

La formación de los NOx es el resultado del nitrógeno que reacciona con el oxígeno en la cámara de combustión. Esta reacción se produce fácilmente con las altas temperaturas existentes en las etapas iniciales del proceso de combustión (cerca de $1900^{\circ} \mathrm{C}$ ), cuando se alcanza la presión máxima y localmente en las zonas en las que el dosado es cercano al estequiométrico, punto en el que se tienen las temperaturas más altas. Mientras la temperatura del cilindro se mantiene suficientemente alta, el NOx que se ha formado comenzará a disociarse de nuevo en nitrógeno y oxígeno. Esto sólo se produce a una velocidad apreciable hasta que la temperatura media de la cámara de combustión disminuye con la presión debido al proceso durante la carrera de expansión. Cuando esto ocurre la disociación cesa y las concentraciones de NOx se congelan. Dicho de otro modo, la velocidad de la refrigeración de las moléculas de NOx, debida a la presencia de gradientes de temperatura elevados entre las diferentes zonas de la cámara de combustión, es la razón principal de que produzcan altos niveles de NOx en motores diésel.

En cuanto a las emisiones de las partículas en los motores diésel, éstas están compuestas principalmente de hidrocarburos sin quemar y material carbonoso hollín. La formación se presenta principalmente en las regiones ricas, a nivel local de la cámara de combustión, donde falta oxígeno para oxidar todos los hidrocarburos del combustible. Algunos hidrocarburos parcialmente quemados pueden condensarse en zonas frías de la cámara, principalmente cerca de la pared del cilindro, formando estructuras de grafito como las de hollín.

Por consiguiente, la duración y las condiciones en las que se desarrolla cada fase de la combustión están directamente relacionadas con los contaminantes emitidos. Si bien, las soluciones (activas o pasivas) que permiten la reducción de contaminantes basan su desarrollo en la información obtenida de la combustión, la futura implementación de dicha información en las estrategias de control de los motores diésel no tiene desprecio.

Sin embargo, debido a la complejidad de los algoritmos de cálculo y la gran cantidad de variables necesarias para lograr un buen diagnóstico de la combustión, surge la necesidad de disponer de herramientas fiables y simples que aporten información de la combustión con los mínimos recursos y en tiempo real. Dicho tiempo real, tiene al menos 
que cumplir con el determinismo exigido por la ECU que, tal y como se comentó en el capítulo 2, no permite ni un tamaño de memoria elevado para registrar datos ni un número elevado de variables medidas necesarias para un preciso diagnóstico de la combustión.

Asimismo, teniendo en cuenta los requisitos del diagnóstico en tiempo real de los parámetros de la combustión, y con el objetivo de facilitar alternativas para dicho diagnóstico, en el presente capítulo, se detallarán algunas herramientas basadas en el análisis de la derivada de la presión en cámara que puedan servir para el cumplimiento de este objetivo. Se presentará el planteamiento de varias metodologías para el diagnóstico, se describirán las herramientas necesarias para su desarrollo, se presentarán los resultados y se discutirán las ventajas y las limitaciones de cada metodología, validando y comparando los resultados con los datos obtenidos con la metodología de diagnóstico de la combustión clásica (CALMEC) mencionada en el apartado 4.3 del capítulo 4.

\subsection{Síntesis y planteamiento de las metodologías}

Como ya se ha comentado en este documento, los modelos de diagnóstico de la combustión más precisos requieren una gran cantidad de variables medidas y otras calculadas para estimar cada fenómeno físico-químico de atomización, evaporación, mezcla, etc. que permita conseguir una descripción global del conjunto del proceso de inyección-combustión diésel. Estos modelos, aun teniendo en cuenta sus limitaciones y las incertidumbres de las hipótesis que incluyen, son insustituibles. Sin embargo, cuando se requiere información en tiempo real sobre eventos particulares a lo largo del fenómeno de la combustión, dichos modelos pueden ser inefectivos, principalmente por dos motivos:

- Desde el punto de vista adquisición de señales, el número de variables a medir se ve incrementado notablemente.

- Desde el punto de vista computacional, la cantidad y la complejidad de operaciones a efectuar es muy elevada, además, la memoria virtual necesaria también es muy elevada. Por ejemplo, para calcular una dFQL aparente a lo largo de un ciclo termodinámico se requieren al menos 4 vectores de datos: presión, volumen, $d p$ y $d V$. Si cada vector se compone de 1440 valores (frecuencia de muestreo angular de $0.5^{\circ}$ ) y cada valor tiene una resolución de 16bits, cada vector de datos ocupará aproximadamente 2.8 Kbytes. Por lo tanto, el resultado y los cuatro vectores de datos ocuparán un total aproximado de 14 Kbytes para cada ciclo. Este dato es despreciable para los ordenadores actuales, sin embargo, para el microcontrolador de las ECUs actuales representa más del $50 \%$ de su memoria RAM interna. Para paliar este problema, se usan las memorias externas del microcontrolador, con el inconveniente de la pérdida de tiempo al acceder a dichas memorias.

Reducir la carga, el tiempo computacional y el número de variables a medir son las principales ventajas que plantean las metodologías que se proponen en este capítulo.

El fundamento principal de estas metodologías se basa en la afirmación de que la liberación de calor produce variaciones bruscas en la señal de presión en cámara y viceversa. De hecho, en numerosos trabajos, se suele separar la presión en cámara en presión correspondiente al ciclo en arrastre y en presión debida a la combustión [1,2]. 
El objetivo de estas metodologías consiste en detectar eventos a lo largo de la derivada de la señal de presión en cámara. A modo de ejemplo, se presenta en la figura (6.1) las señales de la derivada de presión en cámara en arrastre y en combustión.
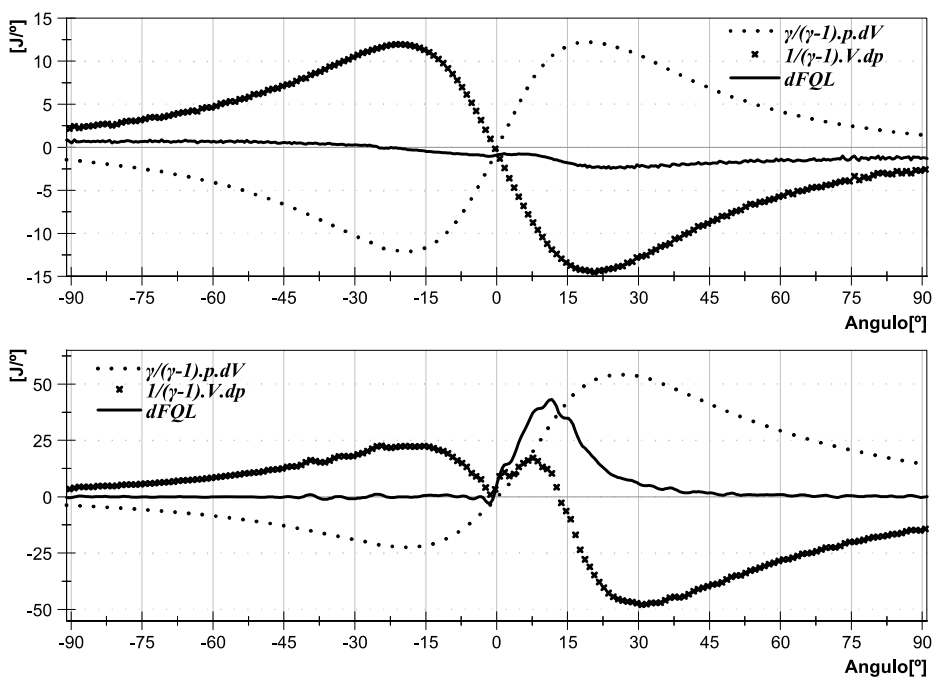

Figura 6.1: Descomposición de la Tasa de liberación de calor aparente para motor en arrastre (arriba) y motor en combustión (abajo) para el mismo régimen de giro.

Como se ve reflejado en la figura (6.1) superior, en caso de arrastre no se aprecian discontinuidades o cambios bruscos en ninguno de los componentes de la $d F Q L$ aparente. Sin embargo, en presencia de la combustión (figura (6.1) inferior) se aprecia que la forma que adapta la $d F Q L$ es básicamente debida a las fluctuaciones que aparecen en el termino $\frac{1}{\gamma-1} \cdot V \cdot \frac{d p}{d t}$. Además, puesto que la variación del volumen es muy lineal (continuidad ecuación del sistema biela-manivela) y la variación del coeficiente isoentrópico se puede considerar lineal (incluso nula si se considera dicho coeficiente constante), el parámetro que más influye en la forma de la $d F Q L$ aparente es la derivada de la presión.

En caso de arrastre, y en los alrededores del PMS, el volumen se puede considerar constante (solo representa un cambio del $2.5 \%$ del volumen total) y la derivada de la presión en cámara es creciente y tiene carácter decreciente con un paso por cero en el punto de máxima presión, debido al paso de compresión a expansión.

En caso de combustión, si bien, la variación del volumen y su derivada, en función del ángulo de giro, no debería alterarse respeto al caso de arrastre, puesto que el volumen está gobernado por el sistema biela-manivela, la energía liberada por la combustión se invierte directamente en producir cambios de la evolución de la presión. De hecho, cabe esperar que a mayor energía liberada por ángulo de giro más rápido incrementara la presión, es decir, valores de la derivada de presión más altos, discontinuidades o cambios bruscos 
en la forma de la derivada de la presión pueden relacionarse con las características de la combustión.

La figura (6.2) representa el comportamiento de la presión, volumen y sus derivadas a lo largo de un ciclo con combustión lo que confirma lo expuesto anteriormente. Es más, se aprecia como cuando ocurre la combustión la derivada de la presión en cámara es la única variable que representa cambios bruscos en su forma. Los rizados en la derivada de la presión pueden verse con claridad mientras que resulta más difícil detectarlos al observar solo la señal de la presión en cámara ya que la integración y el filtrado enmascaran los eventos que si que se observan en la derivada.

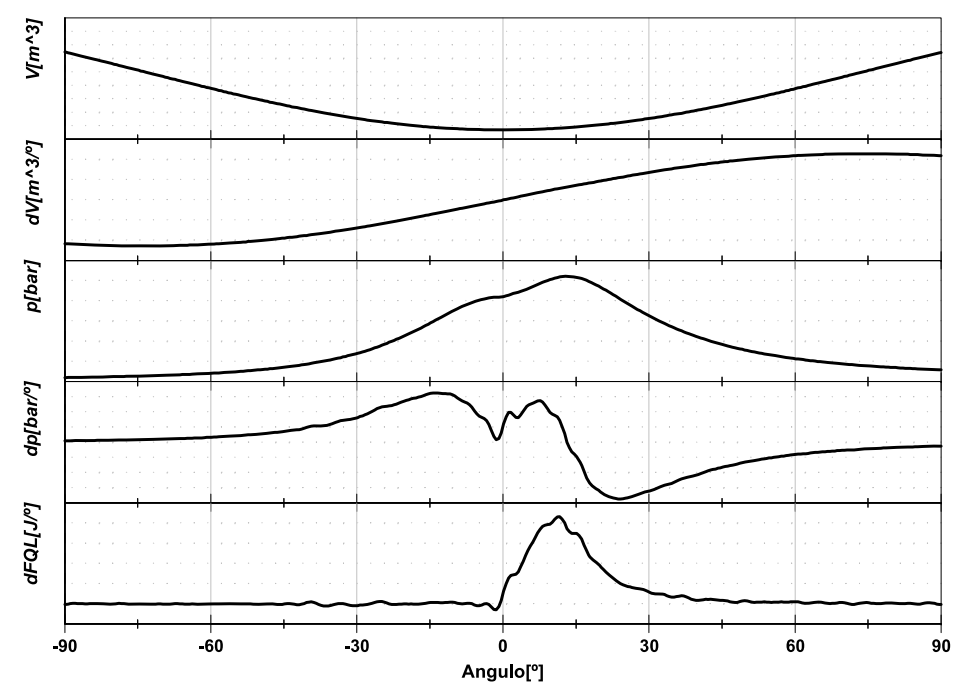

Figura 6.2: Descomposición de las variables que influyen en el cálculo de la $\mathrm{dFQL}$ aparente en presencia de la combustión.

En definitiva, en este capítulo se va a analizar experimentalmente la relación existente entre los parámetros de la combustión y la información extraída de la forma y la dinámica de la señal de la derivada de la presión en cámara. Dicha relación parte del siguiente principio: "A partir del ángulo inicio de la inyección (SoI) cualquier cambio brusco en la forma o en las frecuencias que componen la señal de la derivada de la presión es consecuencia de la variación en la magnitud de la tasa de liberación de calor (dFQL)".

Se plantean dos formas para el estudio de la señal:

- En primer lugar, un estudio en dominio temporal de la derivada de la señal de presión en cámara para extraer los puntos más significativos, como los mínimos y máximos locales, amplitud del rizado y energía de dicha señal y relacionarlos con el SoC y el EoC. 
- En segundo lugar, se plantea un estudio en dominio de frecuencias de dicha señal para localizar puntos donde existen cambios significativos de frecuencia de la misma y relacionarlos con fenómenos de la combustión.

\subsection{Metodología de diagnóstico de la combustión a partir del análisis temporal de la $d p$.}

\subsubsection{Herramientas de análisis de la señal en dominio temporal}

Esta herramienta trata la señal muestra por muestra basándose en criterios estadísticos o puramente matemáticos, como la evaluación de las derivadas para localizar máximos, mínimos y puntos de inflexión de la señal. Dichas localizaciones, que en definitiva, se pretenden identificar de forma automática.

En efecto, cuando se trata de señales continuas equivalentes a funciones temporales, la ciencia matemática define el punto de inflexión de una función como aquel donde la derivada de dicha función cambia de signo. Sin embargo, cuando se trata de señales discretas y en presencia de mucho ruido, no todos los puntos de inflexión de la señal son puntos de inflexión reales. Este aspecto fuerza la utilización de filtros que, en general, producen distorsiones, desfases y pérdida de información relevante contenida en la señal.

Generalmente, se suele analizar la señal muestreada en ventanas móviles, es decir, se analiza la señal en grupos secuenciales de muestras tomadas equiparando cada grupo a una función base o polinomios de grados de complejidad sujetos al criterio del usuario. De esta forma, se suele implementar de forma implícita, un filtrado de la señal y, al mismo tiempo, obtener para cada rango de datos una estimación de la función base que más se ajuste a cada grupo de puntos.

En la práctica, para detectar los puntos de inflexión de una señal se puede utilizar polinomios cuadráticos, asemejando cada secuencia de muestras $\left(\left[\left(i-k, y_{i-k}\right), \cdots,\left(i, y_{i}\right)\right.\right.$, $\left.\left.\cdots,\left(i+k, y_{i+k}\right)\right]\right)$ a una parábola $\left(\widehat{y_{x}}=a \cdot x^{2}+b \cdot x+c\right)$ cuyo coeficientes $(a, b, c)$ son definidos mediante métodos que minimicen el error cometido en la aproximación. Al considerar dicho error como el error cuadrático medio, el método de ajuste, denominado “ajuste por mínimos cuadrados”, busca los parámetros $(a, b, c)$ minimizando la función:

$$
S(a, b, c)=\sum_{x=i-k}^{i+k}\left(\widehat{y_{x}}-y_{x}\right)^{2}=\sum_{x=i-k}^{i+k}\left(a \cdot x^{2}+b \cdot x+c-y_{x}\right)^{2}
$$

El mínimo de la función representada en la ecuación(6.3.1) se alcanza cuando sus derivadas parciales se anulan, es decir, el problema de calcular los coeficientes $(a, b, c)$ se limita a resolver el siguiente sistema:

$$
\left\{\begin{array}{l}
\frac{\partial S}{\partial a}=0=2 \cdot \sum_{i-k}^{i+k}\left(a \cdot x^{2}+b \cdot x+c-y_{x}\right) \cdot x^{2} \\
\frac{\partial S}{\partial b}=0=2 \cdot \sum_{i-k}^{i+k}\left(a \cdot x^{2}+b \cdot x+c-y_{x}\right) \cdot x \\
\frac{\partial S}{\partial c}=0=2 \cdot \sum_{i-k}^{i+k}\left(a \cdot x^{2}+b \cdot x+c-y_{x}\right)
\end{array}\right.
$$

De forma matricial: 


$$
\left[\begin{array}{ccc}
(i+k)-(i-k) & \sum_{i-k}^{i+k} x & \sum_{i-k}^{i+k} x^{2} \\
\sum_{i-k}^{i+k} x & \sum_{i-k}^{i+k} x^{2} & \sum_{i-k}^{i+k} x^{3} \\
\sum_{i-k}^{i+k} x^{2} & \sum_{i-k}^{i+k} x^{3} & \sum_{i-k}^{i+k} x^{4}
\end{array}\right] \cdot\left[\begin{array}{c}
c \\
b \\
a
\end{array}\right]=\left[\begin{array}{c}
\sum_{i-k}^{i+k} y_{x} \\
\sum_{i-k}^{i+k} x \cdot y_{x} \\
\sum_{i-k}^{i+k} x^{2} \cdot y_{x}
\end{array}\right]
$$

Para resolver dicho sistema de ecuaciones, se requieren como mínimo 3 puntos. A medida que aumenta el número de puntos se logra un efecto de filtrado de la señal puesto que todos los puntos se ajustan a la ecuación de la parábola.

Una vez calculados los coeficientes $(a, b y c)$, localizar el punto de inflexión, si existe, en el rango de puntos elegido, se reduce a evaluar la primera y la segunda derivada de la ecuación de la parábola según el criterio:

$$
\begin{cases}x=-\frac{b}{2 \cdot a} & \text { punto de inflexión si } x \in] i-k, i+k[ \\ 2 \cdot a>0 & \text { la parábola presenta un máximo local en } x \\ 2 \cdot a<0 & \text { la parábola presenta un mínimo local en } x\end{cases}
$$

Por lo tanto, las ubicaciones de los puntos de inflexión estimados por este método no corresponden, generalmente, a puntos de datos reales en la señal de entrada muestreada. El punto obtenido puede situarse entre dos muestras reales seguidas, lo que presenta una ventaja cuando la frecuencia de muestreo es relativamente baja.

Este método es altamente fiable aunque tiene un coste computacional de cálculo de matrices elevado. Para reducir este coste computacional de detección de mínimos locales en tiempo real se propone el algoritmo presentado en la figura (6.3), que consiste en lo siguiente:

- A partir del punto de inyección se muestrea y localiza el primer punto $(\alpha)$ cuya valor de $d p$ es inferior a una tolerancia multiplicada por la $d p$ mínima inicializada como el valor de $d p$ en el punto de inyección .

- Se comprueba para los puntos posteriores que el mínimo localizado no haya sido superado, incrementando un contador que en caso de superar el margen de puntos establecido se da por válido el mínimo local localizado.

Se ha considerado un margen de 5 muestras y una tolerancia de 1.05 para los ensayos reales sobre los motores diésel. Aunque dichos parámetros, y principalmente la tolerancia, dependen de la calidad del sistema de adquisición de datos y de la cantidad de ruido presente en la señal de $d p$.

Cuando la señal de $d p$ presenta un ruido considerable, y vista la dificultad del filtrado en tiempo real, para suavizar la señal se considera el uso de un filtro de media móvil centralizada, con ventanas de longitud $N=5$ usando la formula:

$$
\overline{d p_{\alpha}}=\frac{1}{N} \sum_{i=\alpha-\frac{N}{2}}^{\alpha+\frac{N}{2}} d p_{i}
$$

Por lo tanto, para determinar cambios bruscos en la derivada de la presión, y teniendo en cuenta los datos de la inyección (número de inyecciones y puntos de inyección), se han empleado algoritmos de detección de mínimos locales de la señal bruta del sensor 


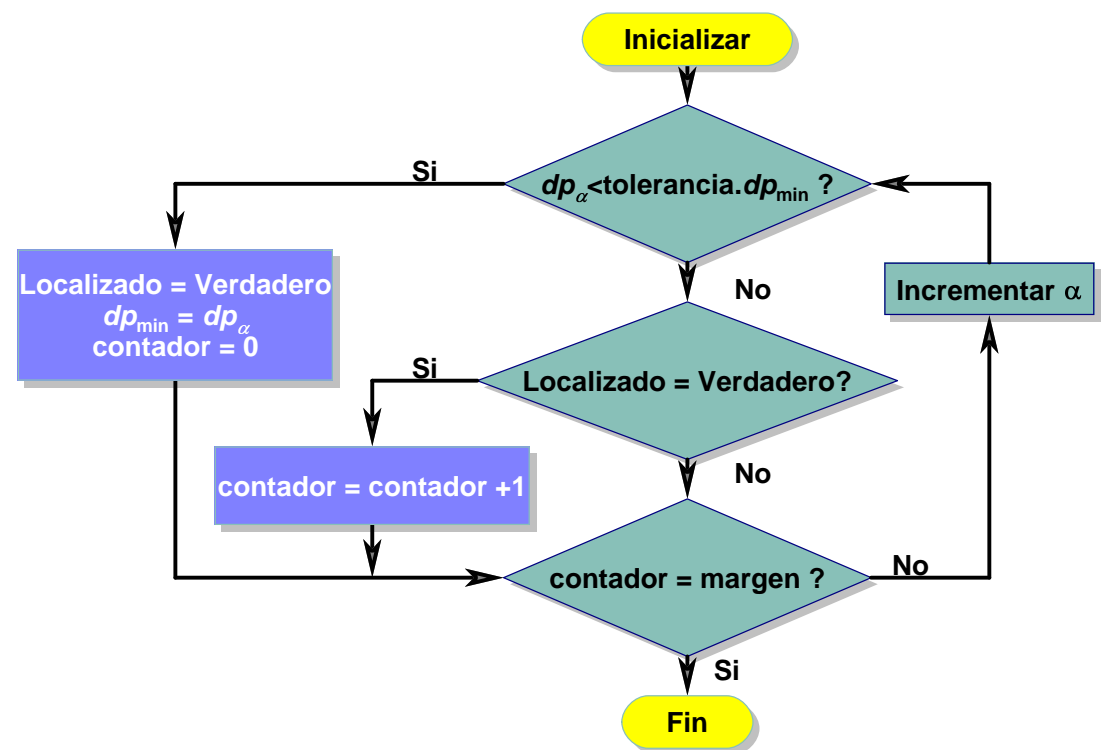

Figura 6.3: Algoritmo básico de detección de mínimos locales

piezoeléctrico, o derivada calculada de la presión medida, usando ventanas con un número adecuado de muestras y una tolerancia adaptada al ruido eléctrico que acompaña a la señal. 


\subsubsection{Aplicación del método basado en el análisis temporal de la derivada de la presión}

En apartados anteriores, se ha desarrollado la idea principal del diagnóstico de la combustión mediante el tratamiento de la señal de la derivada de la presión en dominio temporal. En el presente párrafo se desarrollará la aplicación de dicha idea para motores diésel, estudiando su validez para varios tipos de motores diésel y teniendo en cuenta diferentes puntos de funcionamiento y números de inyecciones.

En la figura (6.4) se representa el caso de una inyección para motor monocilíndrico, la zona sombreada representa el rango comprendido entre el inicio y el final de la inyección ([SoI,EoI]). El ángulo del SoC es el determinado mediante el programa CALMEC y el método de mínimos locales. En dicha figura, la derivada de la presión se obtiene a partir de la señal de presión en cámara promediada y filtrada. Obviamente, el primer mínimo local significativo de $d p$ después del inicio de la inyección corresponde con el SoC estimado mediante la $d F Q L$ de CALMEC. Además, se aprecia que el ángulo de la máxima tasa de liberación de calor, coincide con el pico máximo de $d p$.

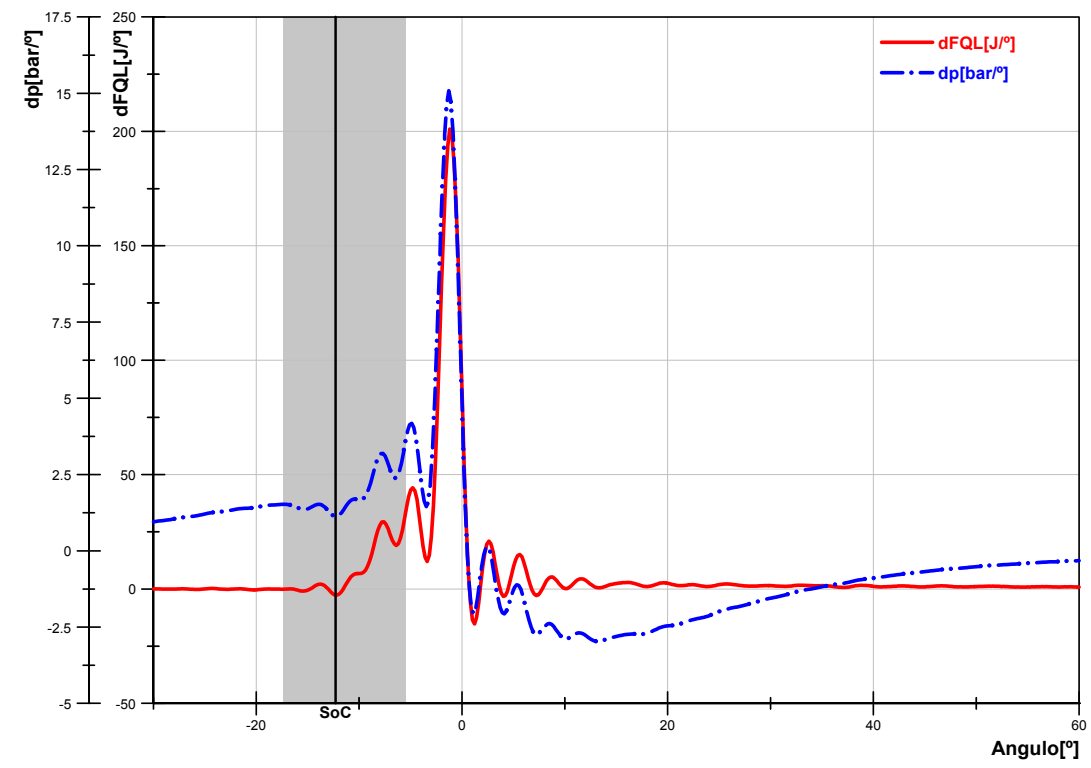

Figura 6.4: Detección del SoC para motor monocilíndrico a (1000rpm, 10.27Nm)

En caso de trabajar con los motores policilíndricos, al usar la $d p$ sin filtrar, el criterio de la detección del SoC por mínimos locales debe ajustarse, considerando una mayor tolerancia al ruido y un filtrado de media móvil centrada, tal y como se mencionó anteriormente (6.3.1). 
La figura (6.5) representa un caso típico de un punto de funcionamiento del motor con una sola inyección. Se aprecia como la $d p$ presenta un rizado considerable a lo largo de la combustión. La evaluación de dicho caso aporta información valiosa para ajuste de los métodos de diagnóstico de la combustión basados en el análisis temporal de la derivada de la presión en cámara.

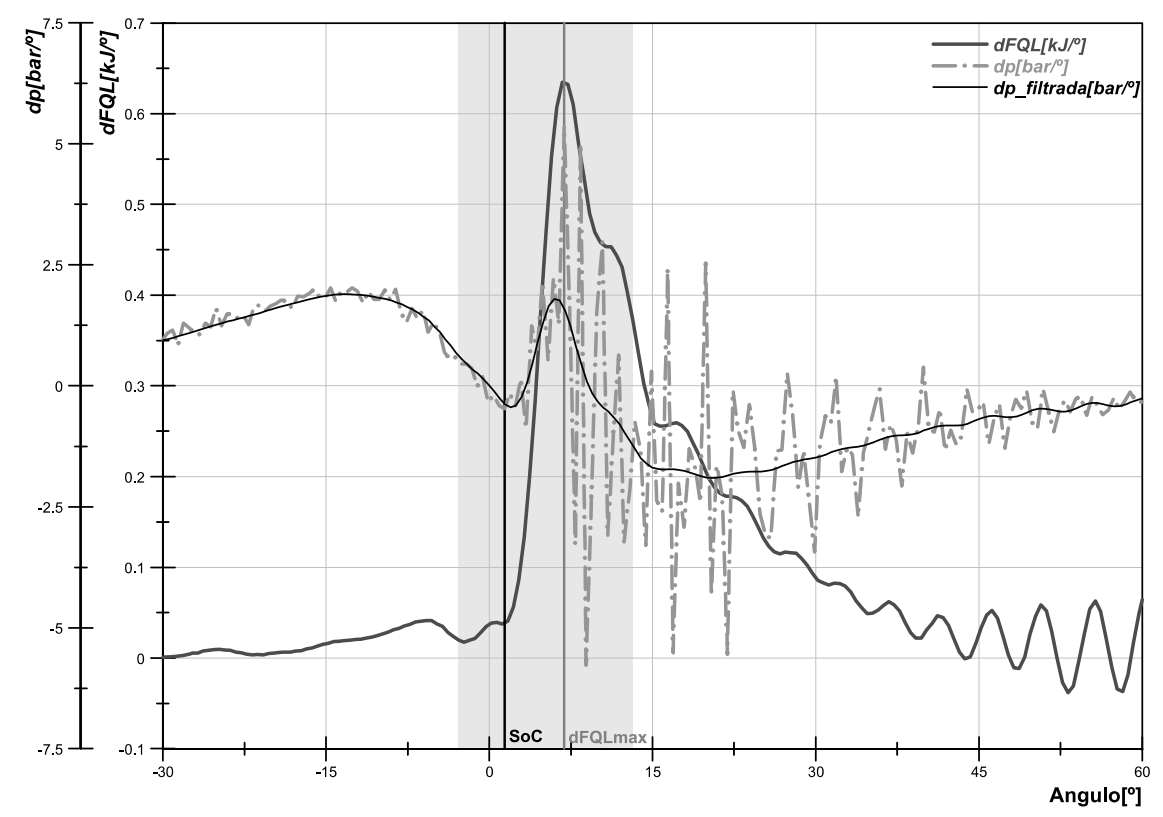

Figura 6.5: Detección del SoC para motor policilíndrico DW10 a (4000rpm, 41.72Nm)

En efecto, y como se comento en tratamiento de la señal de la presión en cámara (apartado 5.1), la desviación típica entre muestras correspondientes al mismo ángulo de giro es mayor para tramos del ciclo donde exista liberación de calor. Las bajas desviaciones típicas se atribuyen al ruido eléctrico y vibraciones, mientras que las altas desviaciones son causadas por la dispersión cíclica del motor y efectos de la combustión sobre el sensor. Estas magnitudes se ven amplificadas al estudiar la desviación de la derivada de la presión aunque no existe proporcionalidad entre las desviaciones de la presión y las de $d p$ :

$$
\sigma_{p}=\sqrt{\frac{\sum_{k=1}^{n_{c}}\left(\overline{p_{\alpha}}-p_{\alpha, k}\right)^{2}}{n_{c}}}<\sigma_{d p}=\sqrt{\frac{\sum_{k=1}^{n_{c}}\left(\overline{d p_{\alpha}}-d p_{\alpha, k}\right)^{2}}{n_{c}}}
$$

Un criterio como el que determina el SoC como el ángulo de giro donde la desviación típica de la derivada de la presión en cámara empieza a incrementarse considerablemente, es igual de válido que el criterio propuesto utilizando mínimos locales. De hecho, se obtienen resultados muy coherentes considerando solo 7 ciclos. Sin embargo, para el 
tratamiento en tiempo real, no es válido esperar varios ciclos para estimar parámetros de la combustión. En este caso, se propone el criterio del rizado medio instantáneo que, en lugar de evaluar la desviación típica en un ángulo de giro para varios ciclos sucesivos, evalúa el rizado medio para varias muestras consecutivas $\left(n_{m}\right)$ usando la ecuación siguiente:

$$
\overline{\sigma_{\alpha^{\prime}}}=\frac{\sum_{k^{\prime}=\alpha-\frac{n_{m}}{2}}^{\alpha}\left|\overline{d p_{\alpha}}-d p_{\alpha, k^{\prime}}\right|}{n_{m}}
$$

donde $\overline{d p_{\alpha}}=\frac{1}{n_{m}} \sum_{k^{\prime}=\alpha-\frac{n_{m}}{2}}^{\alpha+\frac{n_{m}}{2}} d p_{\alpha, k^{\prime}}$ es la media de $d p$ en una ventana de $n_{m}$ muestras centralizada en el ángulo $\alpha$.

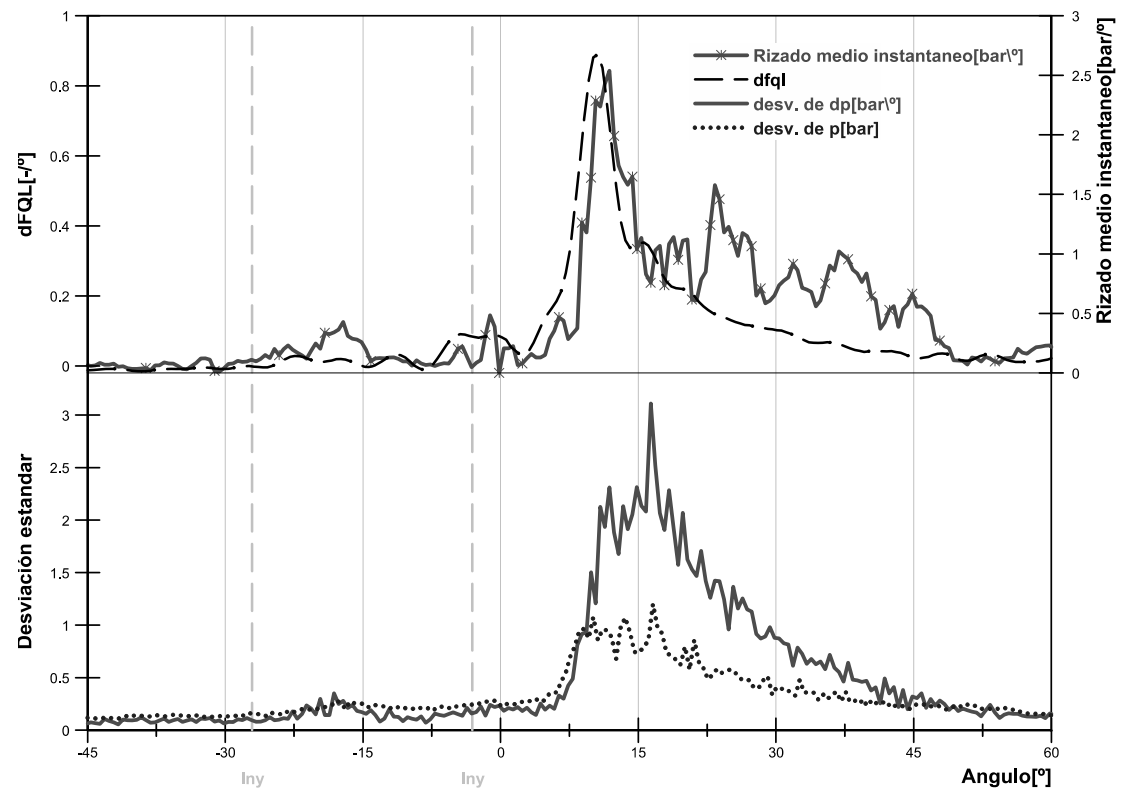

Figura 6.6: dFQL y parámetros estadísticos para la detección de parámetros de la combustión mediante el análisis en el dominio tiempo de la señal de dp.

En la figura (6.6) se aprecia el comportamiento de las desviaciones típicas en la medida de 25 ciclos (parte baja de la gráfica) y el del rizado medio instantáneo para un ciclo cualquiera (parte alta), para un determinado punto de funcionamiento del motor (DW12) donde se producen dos inyecciones por ciclo.

Despreciando el efecto del ruido de fondo de la medida, se puede observar que, al producirse la inyección, el rizado medio instantáneo se altera debido al acoplamiento del ruido eléctrico de la señal de la inyección con la señal de $d p$.

Durante la combustión, las grandes y rápidas alteraciones en la presión, las oscilaciones en el conducto donde está implementado el sensor piezoeléctrico y varios 
fenómenos más, hacen aumentar considerablemente el rizado presente en la señal de $d p$. Los máximos valores se alcanzan alrededor de la máxima $d F Q L$.

La información aportada por $\overline{\sigma_{\alpha}}$ se combinará con el método de detección del SoC a partir de los mínimos locales de $d p$, principalmente para las combustiones piloto donde existe mayor incertidumbre. En este caso, se asignará el SoC al punto donde se localiza un mínimo local seguido por un rizado notable.

A modo de ejemplo, en la figura(6.7) se detectan los mínimos locales A y B, considerando el punto A como SoC de la combustión piloto, puesto que presenta mayor rizado que el punto $\mathrm{B}$, además de ser el punto $\mathrm{A}$ un mínimo local.

Por otra parte, se considera el final de la combustión (EoC) como el punto donde el rizado vuelva a tomar valores parecidos a los correspondientes al ruido de fondo (evaluado antes de las inyecciones) con una tolerancia de ajuste.

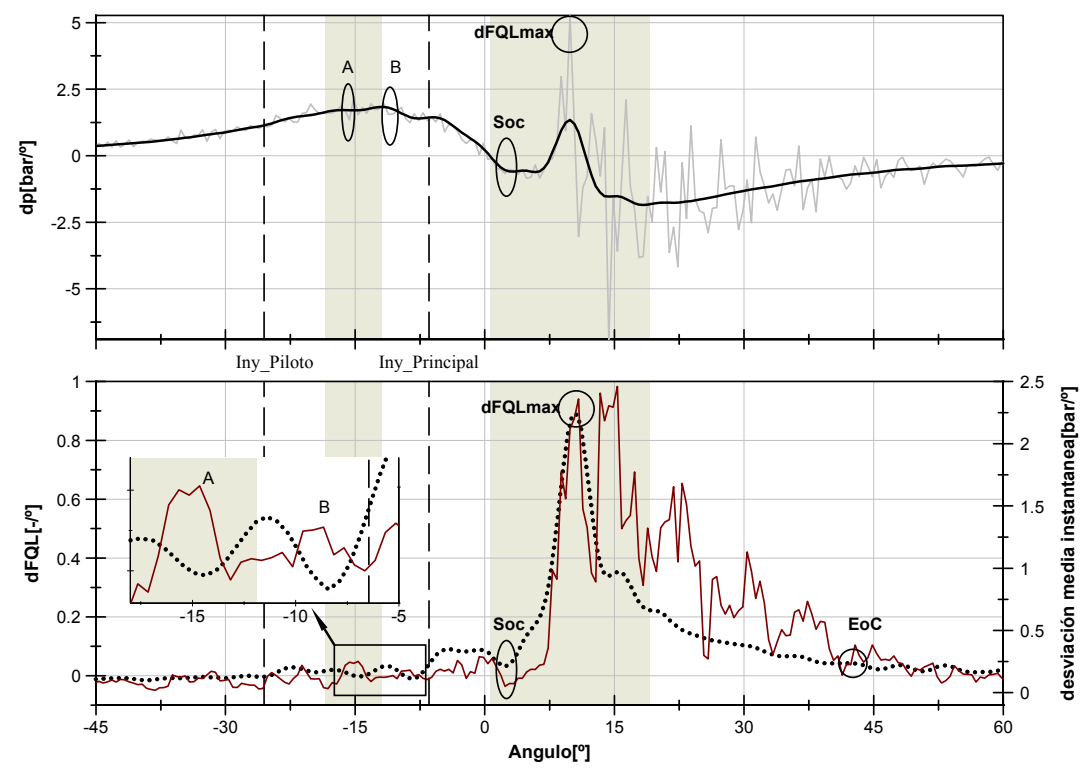

Figura 6.7: Parámetros influyentes en el diagnóstico de la combustión mediante el análisis en el dominio tiempo de la señal de dp (DW12 a 3500rpm, 16.3Nm) 


\subsubsection{Validación, análisis comparativo detección del SoC}

Para validar la metodología de detección del SoC mediante el método basado en el análisis temporal de la derivada de la presión presentado, y al que se va a referir como el método de mínimos locales, se han agrupado los resultados de ensayos sobre diferentes motores en función del número de inyecciones correspondientes a los puntos de funcionamiento a analizar. De esta forma, se podrá evaluar la robustez de la metodología para combustiones piloto y para combustiones principales.

En el caso de varias inyecciones, y debido a la incertidumbre en la localización del SoC de las combustiones piloto, la validación de la metodología de diagnóstico de la combustión a partir del análisis temporal de la $d p$ se efectuara de dos formas:

1. Comparando los resultados obtenidos mediante CALMEC (SoC_CALMEC) y los obtenidos mediante la metodología propuesta (SoC_min.local) aplicada a la señal media sin filtrar .

2. Estudiando la distribución estadística de los resultados de la metodología propuesta aplicada a la señal ciclo a ciclo sin filtrar y comparando con los resultados obtenidos mediante CALMEC.

\subsubsection{Caso de puntos de funcionamiento del motor con una inyección.}

La comparativa entre los resultados de SoC estimados por CALMEC y los estimados por el método propuesto, para varios puntos de funcionamiento de un motor policilíndrico (DW10), se refleja en la figura (6.8).

La desviación presentada para cada punto representa la desviación estándar del error cometido al tratar ciclos sin promediar ni filtrar respecto a los resultados de CALMEC. Se aprecia que, generalmente, a medida que el inicio de la combustión se acerca al PMS la desviación estándar del error se hace más pequeña. Eso es debido a las mayores presiones en el SoC que permiten mejores condiciones de combustión y, por lo tanto, mayor repetitividad en condiciones estables del motor.

Los resultados obtenidos son muy repetitivos para varios tipos de motores y en todas las condiciones de funcionamiento. La tabla (6.1) presenta los parámetros estadísticos del error cometido en la comparativa para los 4 motores reflejados en la tabla 4.1.

\begin{tabular}{|c|c|}
\hline Parámetro & Valor \\
\hline \hline Moda & 0 \\
\hline Media & 0.03 \\
\hline Mediana & 0 \\
\hline Desviación estándar & 0.34 \\
\hline Máximo & 1 \\
\hline $\mathrm{N}^{\circ}$ de puntos & 45 \\
\hline $\mathrm{N}^{\mathbf{o}}$ de ciclos & 1125 \\
\hline
\end{tabular}

Tabla 6.1: Parámetros estadísticos del error en la detección del SoC, caso 1 inyección. 


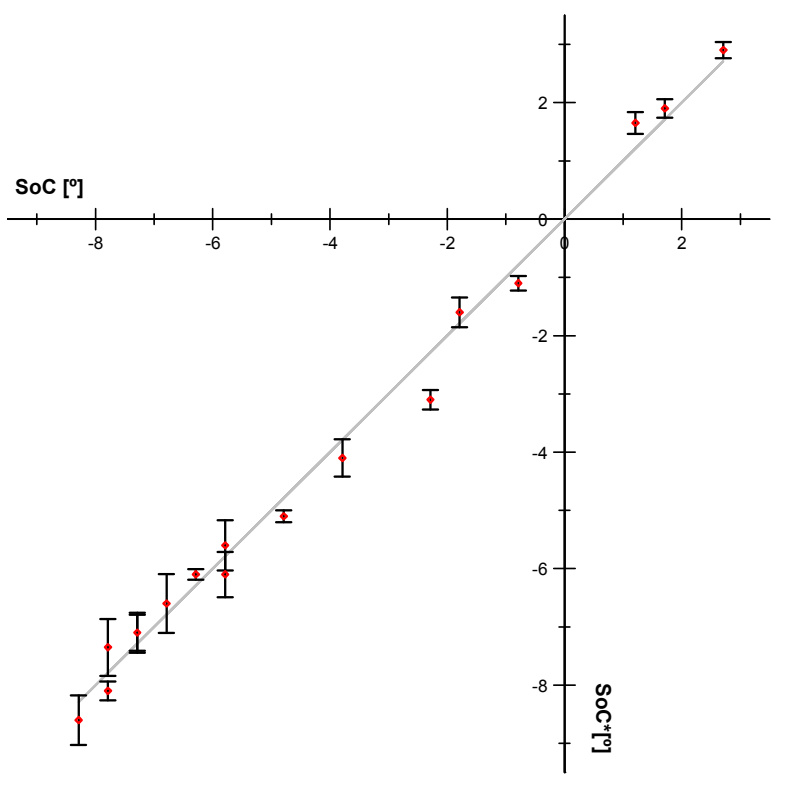

Figura 6.8: Comparativa detección del SoC con CALMEC y con el método de mínimos locales $\left(\operatorname{SoC}^{*}\right)$

\subsubsection{Caso de puntos de funcionamiento del motor con varias inyecciones.}

La figura (6.9) representa, para puntos de funcionamiento del motor DW12 con dos inyecciones, una comparativa entre los resultados obtenidos mediante CALMEC y los obtenidos mediante la metodología propuesta aplicada a la señal media sin filtrar para detectar el SoC de la combustión principal. La media del error cometido es menor de un grado $\left(0.5 \pm 0.74^{\circ}\right)$.

Sin embargo, en la detección del SoC de la combustión piloto, el error cometido es mayor. La figura (6.10) ilustra, de manera efectiva, que los resultados obtenidos se distribuyen alrededor de la línea de igualdad con un error de $1.75 \pm 0.84^{\circ}$.

Aplicando el método en tiempo real y tratando ciclo a ciclo las derivadas de la presión en cámara, se representa mediante las figuras 6.11 y 6.12, para los mismos puntos de funcionamiento del motor DW12 representados en la figuras 6.9 y 6.10, la media y la desviación típica de los resultados para el caso de la combustión principal (figura (6.11) ) y el caso de la combustión piloto (figura (6.12)). 


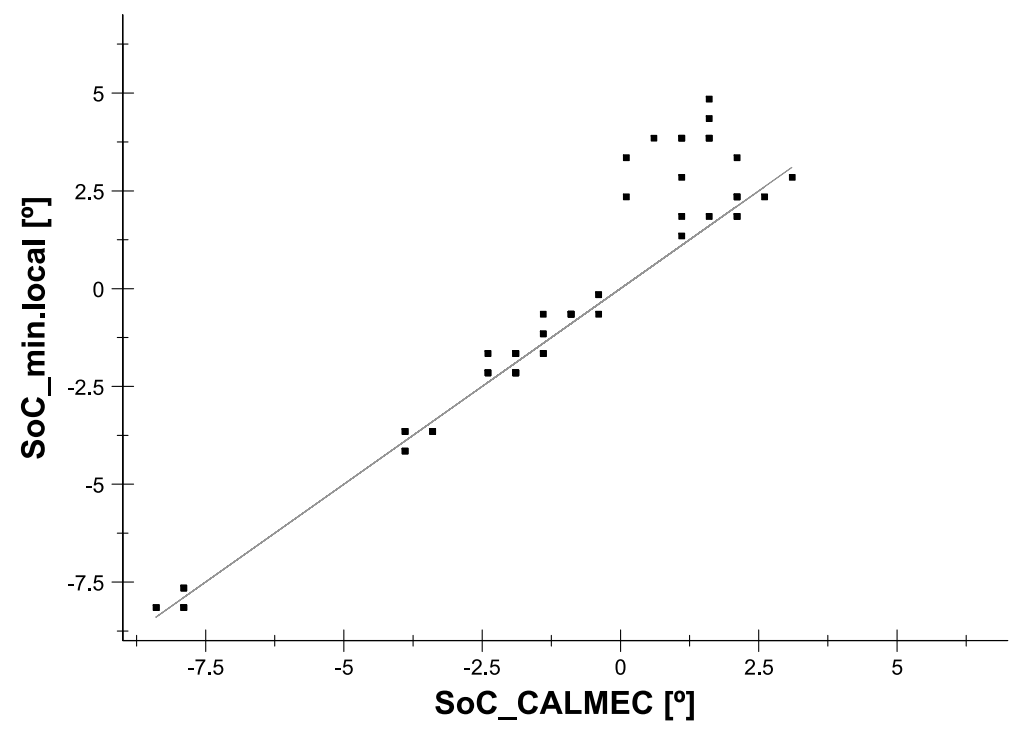

Figura 6.9: Comparativa detección del SoC de la combustión principal con CALMEC y con el método de mínimos locales aplicado al ciclo medio.

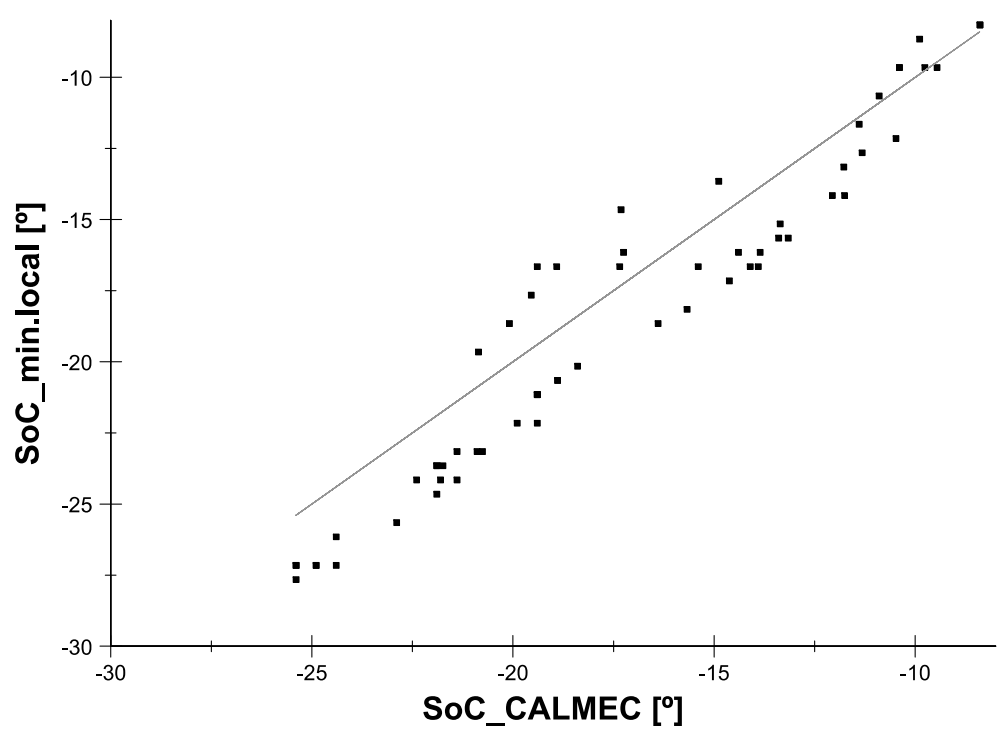

Figura 6.10: Comparativa detección del SoC de la combustión piloto con CALMEC y con el método de mínimos locales aplicado al ciclo medio. 


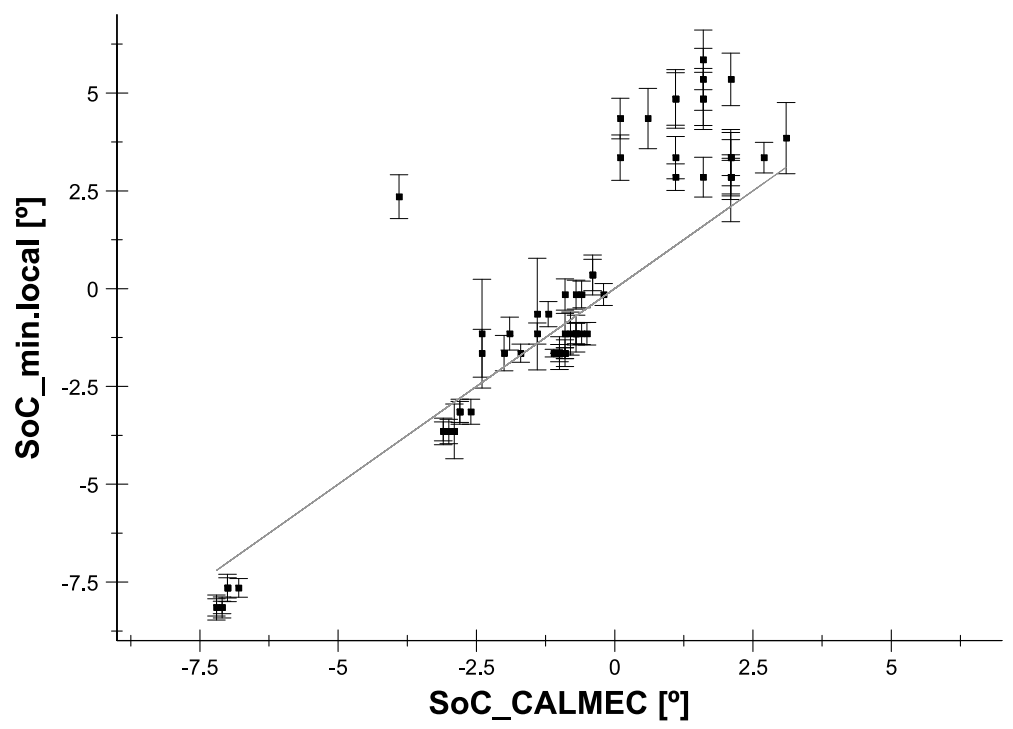

Figura 6.11: Comparativa detección del SoC de la combustión principal con CALMEC y con el método de mínimos locales aplicado ciclo a ciclo.

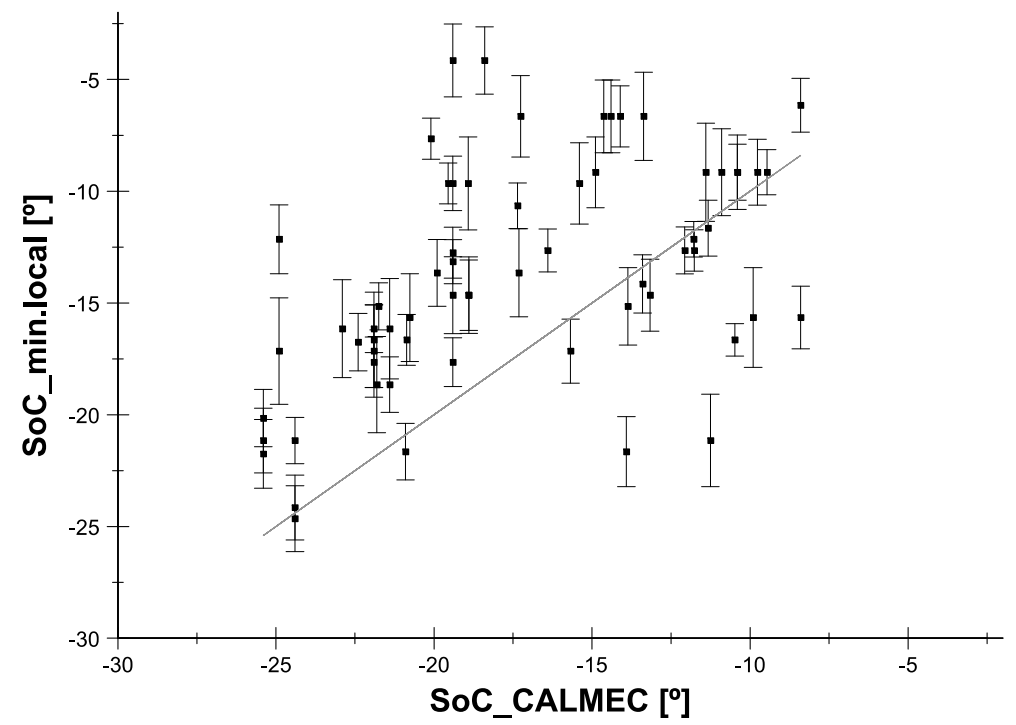

Figura 6.12: Comparativa detección del SoC de la combustión piloto con CALMEC y con el método de mínimos locales aplicado ciclo a ciclo. 
Para el caso del diagnóstico ciclo a ciclo de la combustión principal, la dispersión de los resultados obtenidos es inferior a $1.4^{\circ} \mathrm{y}$, además, la diferencia entre la media de los resultados en un punto de funcionamiento y el resultado aportado por CALMEC es inferior a $1^{\circ}$ en la mayoría de los casos.

Sin embargo, para el diagnóstico ciclo a ciclo de la combustión piloto, la metodología propuesta presenta mayor grado de dispersión para los mismos puntos de funcionamiento (alrededor de $4.13^{\circ}$ para el motor DW12). Además, la media para cada punto de funcionamiento presenta un error significativo respecto a los resultados obtenidos mediante CALMEC.

Para un mejor análisis de los resultados se representan la distribuciones del valor absoluto del error obtenido; comparando, por una parte, el error cometido al tratar el ciclo medio (sin filtrar) del cual se obtiene la tasa de liberación de calor mediante CALMEC, y por otra parte el error cometido al tratar la $d p$ ciclo a ciclo, comparando con la localización del SoC conseguida mediante el análisis (mediante la metodología propuesta) del ciclo medio.

La figura (6.13) confirma que los resultados relativos a la combustión principal son altamente precisos, independientemente de si se trata el ciclo medio (figura (6.13a)) o si se hace el tratamiento ciclo a ciclo (figura (6.13b)).

Sin embargo, para la combustión piloto hay una gran diferencia entre la distribución del error al tratar ciclo a ciclo o el ciclo medio. Este efecto puede explicarse por los siguientes motivos:

- La baja precisión al tratar el ciclo medio (figura(6.14a)) es debida a la incertidumbre en la detección del SoC por cualquiera de los dos métodos comparados entre sí. Las condiciones desfavorables para la combustión del combustible de las inyecciones piloto son muy probables. Incluso en el caso de combustiones lentas se dificulta mucho la detección del SoC para cualquier método basado en la presión o su derivada. Aun así, errores inferiores a $4^{\circ}$ de giro del motor se consideran aceptables (para combustiones débiles).

- En cuanto se hace la comparativa ciclo a ciclo (figura (6.14b)), se aprecia que en un $43 \%$ de los casos el error es inferior a $1^{\circ}$, lo que da robustez al método. Aunque, aparecen errores de gran magnitud (superiores a $4^{\circ}$ ). Este efecto es indicativo y está justificado por la dispersión cíclica del motor, pues aumenta el número de ciclos en los que el tratamiento de la derivada de presión aporta una detección de SoC bastante repetitiva y sin embargo, existen algunos ciclos en los que no se detecta el SoC o se obtienen valores suficientemente alejados de la media.

La tabla (6.2) representa los parámetros estadísticos de la dispersión del error para las dos metodologías para combustiones piloto. La lectura de dicha tabla confirma que:

- El método de los mínimos locales proporciona una diferencia más repetitiva con respecto al método de $C A L M E C$ de $1.75^{\circ}$ con una desviación típica de $0.83^{\circ}$ al tratar el ciclo medio correspondiente al punto de funcionamiento del motor.

- El valor más repetitivo, cuando se comparan los resultados del tratamiento de un ciclo cualquiera mediante la metodología de mínimos locales con los resultados 


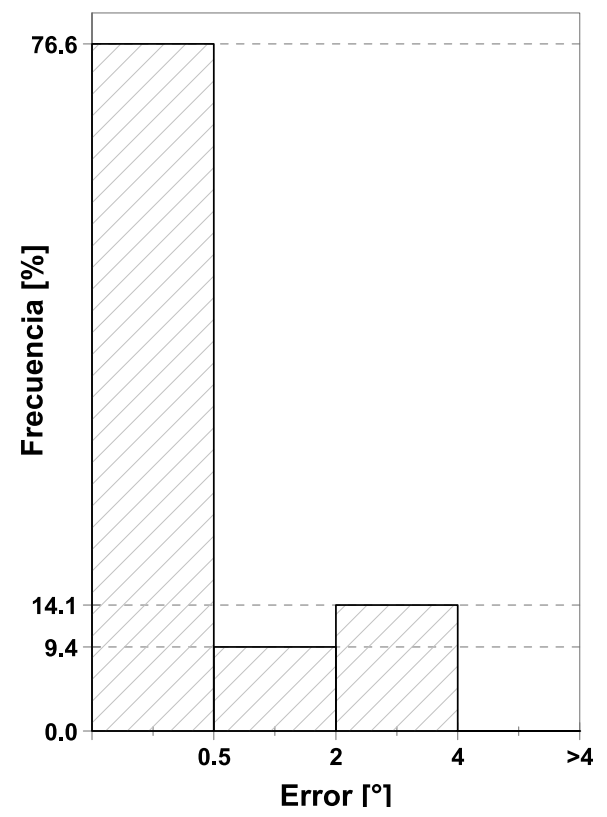

(a) Histograma del error al tratar el ciclo medio

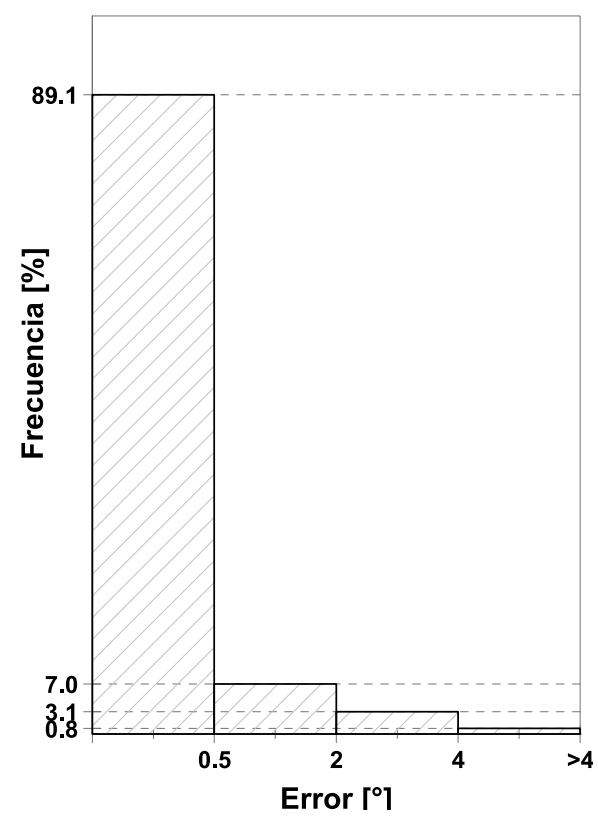

(b) Histograma del error al tratar ciclo a ciclo

Figura 6.13: Distribución del error en el diagnóstico del SoC de la combustión principal, puntos de funcionamiento del motor con 2 inyecciones.

del análisis con CALMEC del ciclo medio de presión en cámara, es de $2.25^{\circ}$ con una desviación típica de $3.12^{\circ}$. Valores altos, justificados por la diferencia entre las señales ciclo a ciclo y la señal del ciclo medio filtrado para el análisis mediante $C A L M E C$, por la dispersión cíclica del motor y la debilidad de muchas combustiones piloto.

- Al comparar los resultados entre el análisis del ciclo medio y un ciclo cualquiera, el valor de la diferencia más repetitivo es cero, aunque la desviación estándar es del orden de la media $\left(3.77^{\circ}\right)$. Esto se debe a que los valores extremos son altos $\left(15^{\circ}\right)$ y ocurren en ausencia de localización del SoC por uno de los dos métodos aplicados. 


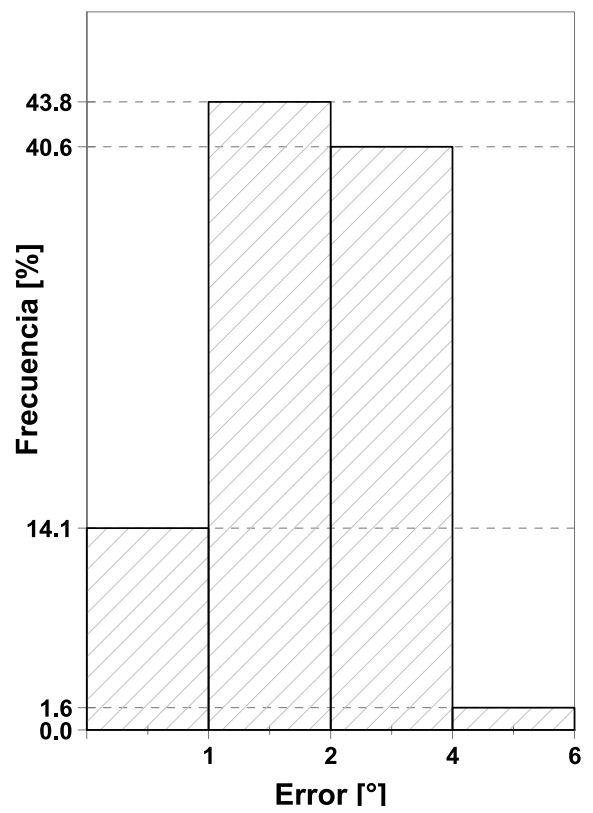

(a) Histograma del error al tratar el ciclo medio

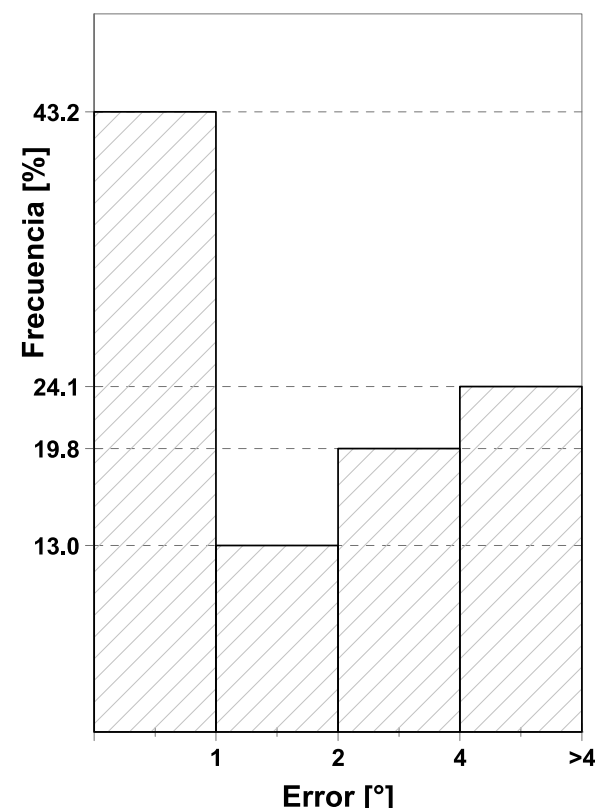

(b) Histograma del error al tratar ciclo a ciclo

Figura 6.14: Distribución del error en el diagnóstico del SoC de la combustión piloto , puntos de funcionamiento del motor con 2 inyecciones.

\begin{tabular}{|c|c|}
\hline Parámetro & Valor \\
\hline \hline Moda & 1.75 \\
\hline Media & 1.85 \\
\hline Mediana & 1.75 \\
\hline Desviación estándar & 0.834 \\
\hline Máximo & 5.10 \\
\hline $\mathrm{N}^{\circ}$ de puntos & 128 \\
\hline
\end{tabular}

(a) Ciclos medios

\begin{tabular}{|c|c|c|}
\hline Parámetro & CALMEC & min. local \\
\hline \hline Moda & 2.25 & 0 \\
\hline Media & 3.64 & 4.11 \\
\hline Mediana & 3.26 & 2.45 \\
\hline Desviación estándar & 3.12 & 3.77 \\
\hline Máximo & 14.75 & 15 \\
\hline $\mathrm{N}^{\mathbf{o}}$ de ciclos & 3200 & 3200 \\
\hline
\end{tabular}

(b) Ciclo a ciclo

Tabla 6.2: Parámetros estadísticos del error en la detección del SoC de la combustión piloto, caso 2 inyecciones. 
En caso de inyecciones piloto múltiples, los resultados anteriores son repetitivos. En efecto, en la figura (6.15) se representan los resultados de comparación del método de mínimos locales y el de CALMEC para el caso de 3 inyecciones.

Para la primera inyección piloto (la más lejana del PMS), el histograma es parecido al obtenido en caso de puntos con dos inyecciones, aunque aparecen casos con diferencia superior a 6 grados, debido a que se trata de inyecciones mucho más avanzadas. Sin embargo, el error disminuye al comparar los resultados de ciclos entre sí para el mismo punto de funcionamiento.

Para las segundas combustiones, los resultados de los dos métodos son más parecidos (62\% de los casos presentan una diferencia inferior a $0.5^{\circ}$ ) y, al tratar ciclo a ciclo, los resultados son coherentes teniendo en cuenta la dispersión cíclica del motor.

En cuanto a la combustión principal, no cabe duda de que con los dos métodos se obtienen datos similares, un $82 \%$ de repetitividad con diferencias menores de $1^{\circ}$, al tratar los ciclos medios, y mayor del $87 \%$ al tratar ciclo a ciclo. 


\section{Histograma del error respecto al ciclo medio}

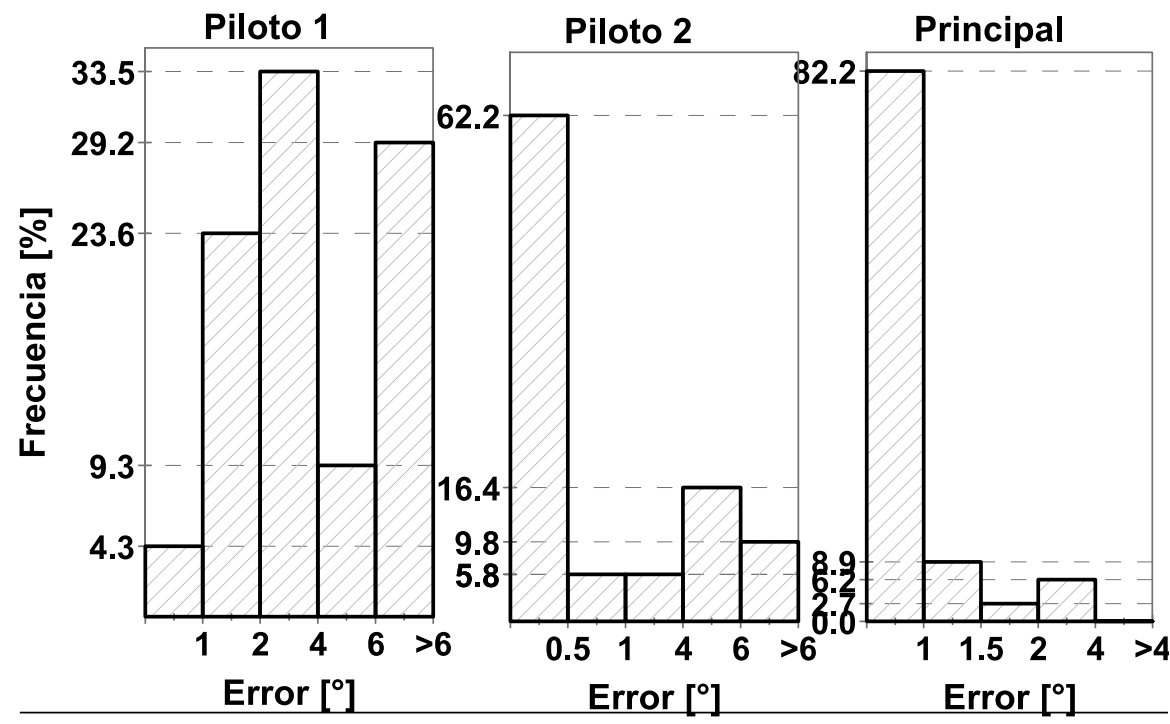

\section{Histograma del error ciclo a ciclo}

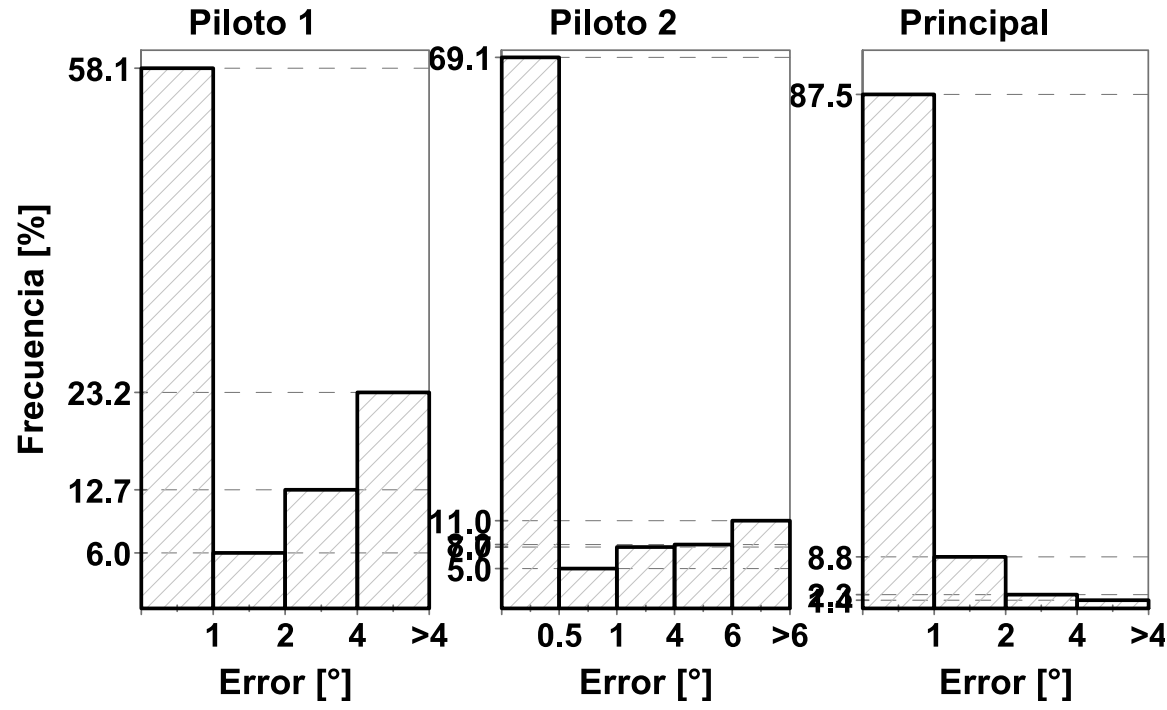

Figura 6.15: Distribución del error de comparación entre CALMEC y min local, puntos de funcionamiento con 3 inyecciones 
Para un mayor detalle en el análisis de los resultados, en el caso de 3 inyecciones, la tabla (6.3) detalla el análisis estadístico de los resultados y confirma la viabilidad del método de diagnóstico del SoC basado en la localización de los mínimos locales de la derivada de la presión.

\begin{tabular}{|l|c|c|c|}
\hline Parámetro & Piloto 1 & Piloto 2 & Principal \\
\hline \hline Moda & 2.25 & 2.25 & 0.25 \\
\hline Media & 3.84 & 2.20 & 0.65 \\
\hline Mediana & 3.11 & 0.25 & 0.25 \\
\hline Desviación estándar & 2.68 & 3.35 & 0.74 \\
\hline Máximo & 10.29 & 13.68 & 3.75 \\
\hline $\mathrm{N}^{\mathbf{o}}$ de puntos & \multicolumn{3}{|c|}{292} \\
\hline
\end{tabular}

(a) Ciclos medios

\begin{tabular}{|l|c|c|c|c|c|c|}
\multicolumn{1}{c}{} & \multicolumn{2}{c}{ Piloto 1 } & \multicolumn{2}{c|}{ Piloto 2 } & \multicolumn{2}{c|}{ Principal } \\
\hline Método & CALMEC & min.local & CALMEC & min.local & CALMEC & min.local \\
\hline \hline Moda & 1.25 & 0.5 & 0.25 & 0 & 0.25 & 0.5 \\
\hline Media & 5.72 & 3.3 & 3.48 & 1.82 & 1.11 & 0.8 \\
\hline Mediana & 4.22 & 1.5 & 1.25 & 0.5 & 0.75 & 0.5 \\
\hline $\begin{array}{l}\text { Desviación } \\
\text { estándar }\end{array}$ & 4.73 & 3.6 & 3.87 & 3.16 & 1.77 & 1.07 \\
\hline Máximo & 16.75 & 15 & 20.65 & 17.1 & 16.2 & 14.5 \\
\hline N $^{\mathbf{0}}$ de ciclos & 11700 & 11700 & 11700 & 11700 & 11700 & 11700 \\
\hline
\end{tabular}

(b) Ciclo a ciclo

Tabla 6.3: Parámetros estadísticos del error en la detección del SoC, puntos de funcionamiento con 3 inyecciones.

Las distribuciones estadísticas obtenidas en este apartado se corresponden a los motores diésel M9R, DW12 y DW10. Para dichos motores se han presentado los resultados relativos a más de 253 puntos de funcionamiento obteniendo resultados satisfactorios con motores, condiciones de funcionamiento y sistemas de adquisición diferentes. 


\subsubsection{Validación, análisis comparativo detección del EoC}

El método de detección del EoC, a partir del análisis temporal de la señal de $d p$, considera el EoC como el ángulo donde la señal de $d p$ empieza a tener un rizado despreciable que, para el caso de tiempo real, corresponde al rizado medio instantáneo calculado antes de las inyecciones.

La detección del EoC a partir de la ley de liberación de calor es muy sensible al tratamiento de la señal de la presión en cámara. El estudio del efecto del promediado sobre la $d F Q L$ efectuado en el apartado 5.2.1 demostró que a partir del promediado de 20 ciclos el error típico cometido en el cálculo de la $d F Q L$ mediante $C A L M E C$ es inferior a $0.1 \mathrm{~J} /{ }^{\circ}$. Este error presenta el valor máximo obtenido durante un ciclo termodinámico y se suele localizar en la zona de máxima $d F Q L$. Sin embargo, la acumulación de todos los errores al integrar la $d F Q L$ hace que el error típico cometido en el cálculo de la $F Q L$ cobre mayor importancia al final de las combustiones. Dicho error, aunque sea pequeño (inferior al $0.25 \%$ ), afecta considerablemente la localización del EoC.

En el caso del diagnóstico en tiempo real, y pese al filtrado, la diferencia entre las $F Q L$ de ciclos consecutivos presenta una dispersión considerable. La figura (6.16a) muestra como en los ángulos correspondientes a fracciones quemadas entre el $60 \%$ y el $95 \%$ se tienen las mayores desviaciones típicas. El efecto de esta dispersión puede observarse en la figura (6.16b), donde se aprecia que, para el caso del ángulo correspondiente a una fracción quemada del $95 \%$, el rango de ángulos correspondientes varia del orden de $11.5^{\circ}$ con una desviación típica de $3.1^{\circ}$, aunque, para dicho rango de ángulos, la variación de la $F Q L$ tiene una desviación típica de $0.4 \%$ en un rango de valores entre 93.4 y $96.6 \%$.

Descartando la capacidad de localizar el EoC de las combustiones piloto, se plantea ahora el análisis comparativo entre los resultados obtenidos a partir de ley de liberación de calor $(F Q L)$ calculada mediante CALMEC y el método basado en la estimación del rizado medio instantáneo en las derivadas de la presión ciclo a ciclo.

El análisis comparativo de los resultados de la localización del EoC se puede plantear de dos formas distintas:

- Comparando los EoC estimados con cada método, fijando para el cálculo de CALMEC el EoC como el ángulo donde se ha liberado el $90 \%$.

- Estudiando los parámetros estadísticos de la dispersión del calor liberado obtenido con CALMEC en los EoC obtenidos por el análisis temporal de la $d p$.

Dada la sensibilidad de la estimación del EoC mediante CALMEC, para la validación de los resultados obtenidos con cada metodología se aceptarán las estimaciones del EoC cuya dispersión máxima (en el mismo punto de funcionamiento) sea inferior a $9^{\circ}$ y donde se manifieste una dispersión máxima en la fracción liberada de calor inferior al 2.5\% estimada mediante CALMEC a partir del ciclo medio. 


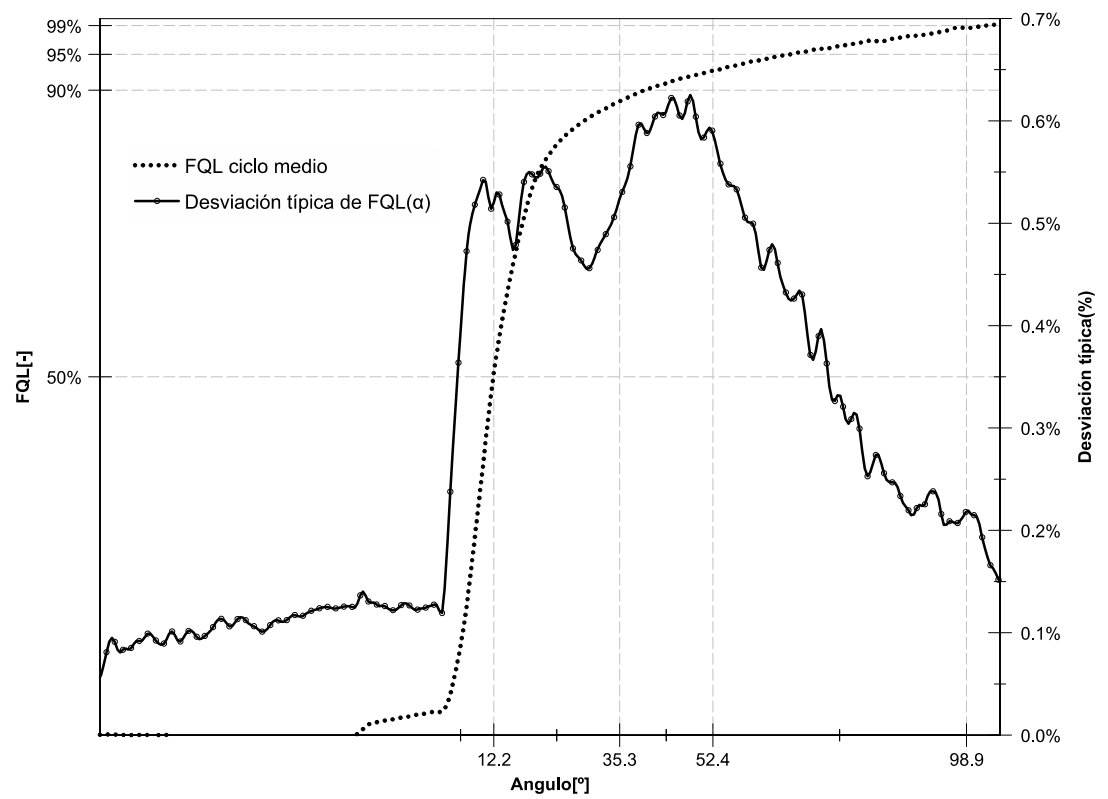

(a) Desviación típica de la $F Q L$.

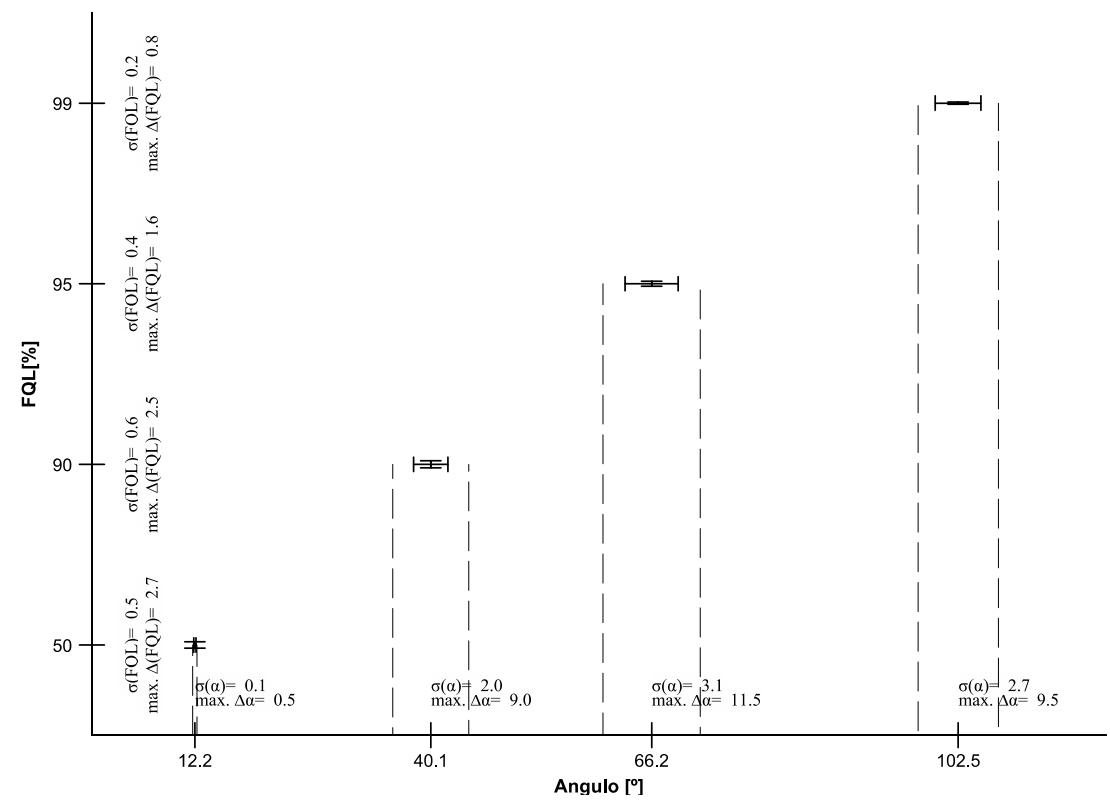

(b) Sensibilidad de la detección del EoC con CALMEC.

Figura 6.16: Efecto de la dispersión entre ciclos en la localización del EoC. 
La figura (6.17) presenta la distribución estadística de los resultados obtenidos en más de 500 puntos de funcionamiento de varios motores (DW10, DW12, DV6 y M9R). Se comprueba que, efectivamente, los resultados obtenidos tienen un rango de variación inferior a $9^{\circ}$ en el $86 \%$ de los casos, en el mismo punto de funcionamiento del motor, y la distribución del calor liberado en los ángulos de EoC estimados tiene una media de $94.6 \%$ con una desviación de $1.25 \%$.

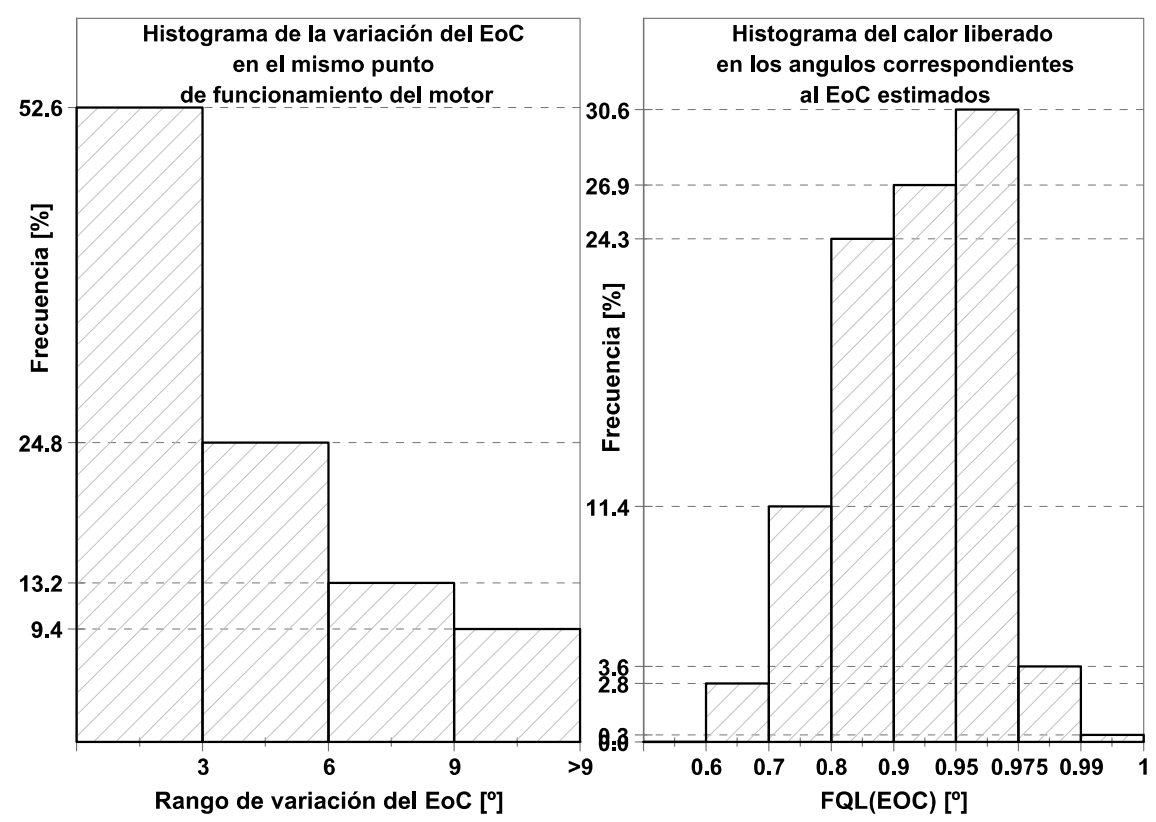

Figura 6.17: Validación de la estimación del EoC mediante análisis temporal de la señal de dp.

La variación significativa en la tasa de liberación de calor, calculada a partir del ciclo medio tratado, es debida a que el método presentado usa el criterio de: "el EoC es el ángulo a partir del cual el calor liberado por la combustión deja de ser influyente en la variación de la presión”. Por otra parte, el ángulo del EoC estimado por los métodos tradicionales tiene en cuenta todo el calor liberado durante todo el ciclo y el método propuesto sólo el correspondiente a la combustión principal. 


\subsubsection{Validación, análisis comparativo detección del ángulo de máxima dFQL}

La magnitud del máximo gradiente de presiones tiene un amplio uso en el estudio de vibraciones y ruidos de combustión [2]. Sin embargo, relacionar el ángulo donde se produzca dicho máximo gradiente con parámetros relacionados con la combustión no es tarea sencilla.

A priori, cabe esperar que el dpmax se produzca cuando haya mayor liberación de calor o, lo mismo, cuando la velocidad de la combustión es máxima. Es decir, cuando la segunda derivada de la $F Q L$ sea nula.

En los motores convencionales, el ángulo de máxima $d F Q L$ suele producirse después del punto muerto superior y como máximo hasta unos $25^{\circ}$ después. A modo de aproximación, y teniendo en cuenta la derivada de la tasa aparente del calor liberado, con un coeficiente politrópico $\gamma$ constante, obtenemos:

$$
d^{2} F Q L=0=d\left(\frac{\gamma}{\gamma-1} \cdot p \cdot \frac{d V}{d t}\right)+d\left(\frac{1}{\gamma-1} \cdot V \cdot \frac{d p}{d t}\right)
$$

A partir del punto muerto superior, la primera parte de la suma $d\left(\frac{\gamma}{\gamma-1} \cdot p \cdot \frac{d V}{d t}\right)$ es siempre positiva (expansión y presión positiva creciente) mientras no se alcance la presión máxima. Por lo tanto, para que se anule la segunda derivada de la $F Q L, \frac{1}{\gamma-1} \cdot V \cdot \frac{d p}{d t}$ tiene que tomar valores negativos y en consecuencia $\frac{d p}{d t}$ tiene que ser decreciente.

Es decir, después de cada máximo local de $d p\left(d\left(\frac{1}{\gamma-1} \cdot V \cdot \frac{d p}{d t}\right)=0\right)$ que se produzca después del punto muerto superior, debe producirse un máximo local en la $d F Q L$. Dicho criterio viene ilustrado en la figura (6.18).

Aproximar el ángulo de dpmax local al ángulo de máxima $d F Q L$ tiene, en la mayoría de los casos, un error despreciable para aplicaciones de diagnóstico en tiempo real, como se comprobará en adelante. Dicho error se puede minimizar teniendo en cuenta el ángulo donde se produzca el dpmax y el ángulo de inicio de la combustión.

Tener en cuenta el ángulo donde se produzca el dpmax viene justificado por la magnitud del componente $d\left(\frac{\gamma}{\gamma-1} \cdot p \cdot \frac{d V}{d t}\right)$ de la ecuación (6.3.2), que se hace mayor a medida que se aleja del PMS.

De hecho, cuando existen varios máximos locales en la $d p$, detectar el ángulo del máximo absoluto de la $d F Q L$ en un ciclo termodinámico requiere una corrección de la $d p$ en función del ángulo de giro. El estudio paramétrico del efecto del avance de la inyección sobre la localización del $d F Q L \max$, reflejado en la figura (6.19), muestra como en los casos A y B el ángulo de la dFQLmax ocurre después del segundo máximo local de la $d p$. Dicho segundo máximo local de la $d p$, aun teniendo una magnitud inferior al primer máximo de la $d p$, el efecto relacionado con el ángulo de giro tiene mayor peso en la zona de los segundos picos. 


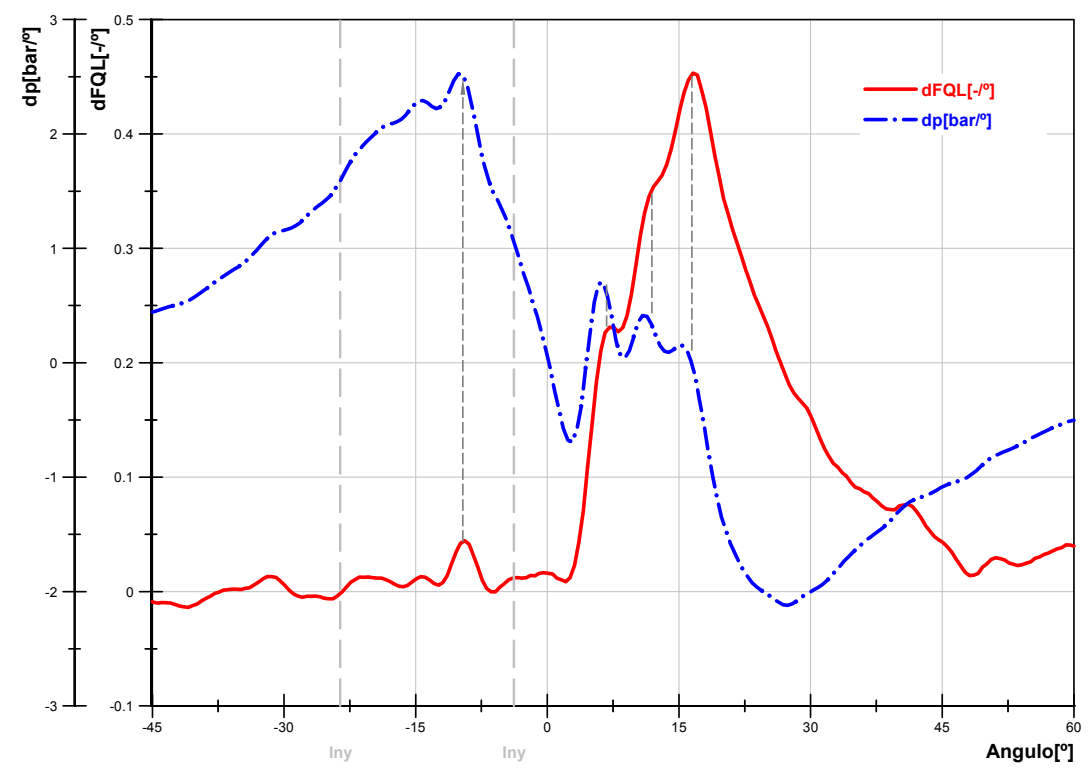

Figura 6.18: Aproximación del ángulo de máxima $\mathrm{dFQL}$ al ángulo de máxima dp local.

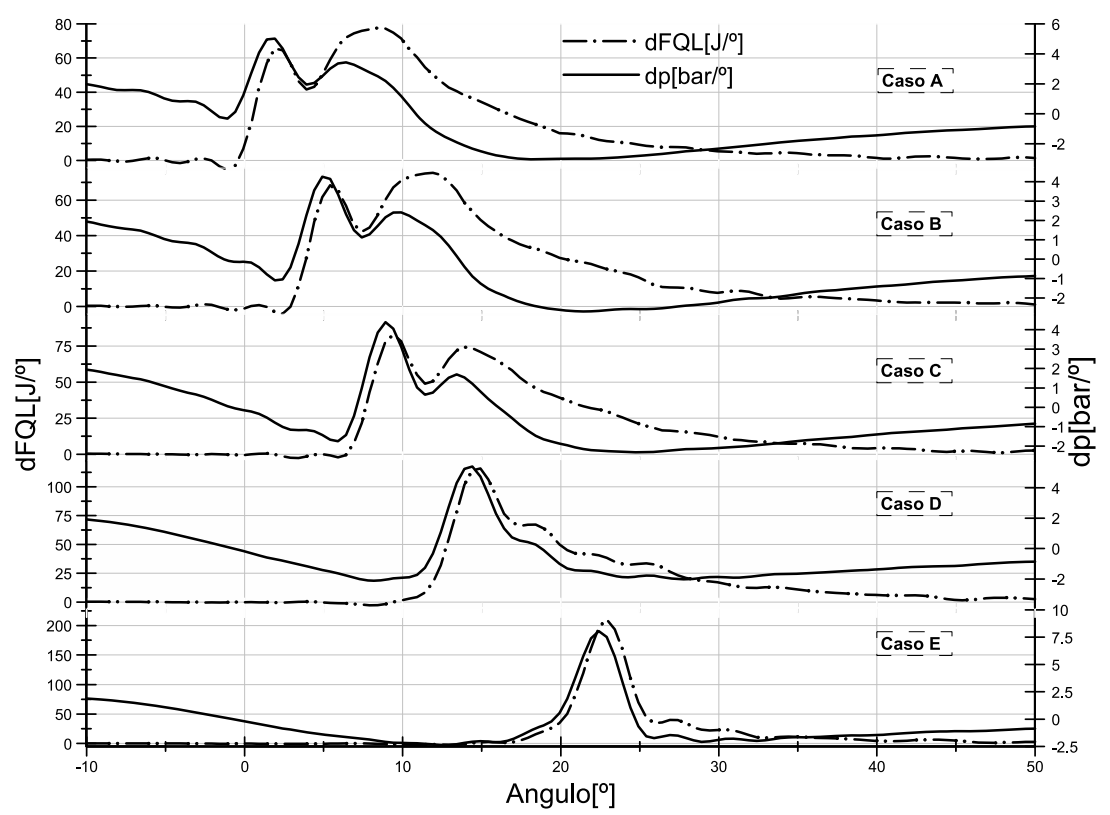

Figura 6.19: Efecto del avance de la inyección sobre el ángulo de dFQLmax.. 
La figura (6.20) ilustra, para el mismo estudio paramétrico anterior, cómo los máximos locales de la $d p$ corregida en función del ángulo de giro $((\mid$ Angulo $\mid+1) \cdot d p)$ se aproximan notablemente a los picos máximos de la $d F Q L$. En este caso, la ecuación propuesta para la corrección $(\mid$ Angulo $\mid+1)$ considera un efecto no nulo en el PMS y que el efecto del incremento de $\left(\frac{\gamma}{\gamma-1} \cdot p \cdot \frac{d V}{d t}\right)$ es lineal. Aún siendo esta consideración incierta, y pudiendo ser mejorada usando una aproximación lineal o polinomial de $\left(\frac{\gamma}{\gamma-1} \cdot \frac{d V}{d t}\right)$, se ha optado por su uso debido a su sencillez y fiabilidad experimental. De otra forma, si se recure al cálculo del volumen, a su derivada y a la presión, se acabará haciendo un cálculo de la $d F Q L$, cuando lo que se pretende es obtener resultados satisfactorios directamente a partir del análisis temporal de la derivada de la señal de presión en cámara con los mínimos recursos computacionales.

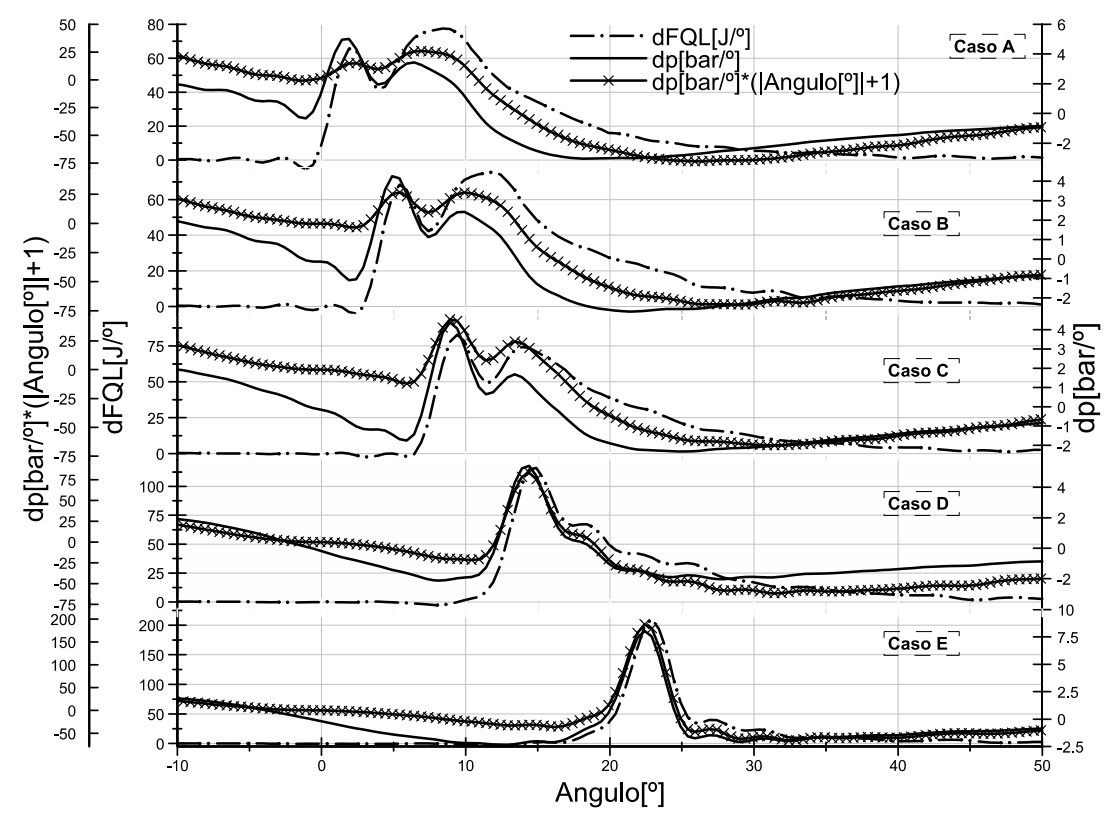

Figura 6.20: Efecto de la corrección de dp sobre la estimación del ángulo de dFQLmax.

En cuanto al inicio de la combustión, su influencia radica en que el ángulo de máximo $d p$ a localizar se produce después del SoC. En definitiva, el criterio utilizado en la estimación del ángulo de $d F Q L \max$ ha sido:

- En caso del SoC antes del PMS, el ángulo de $d F Q L$ max es el ángulo de presión máxima $(d p=0)$.

- En caso contrario, el ángulo de dFQLmax es el ángulo de $((|\alpha|+1) \cdot d p)_{\max }$.

Con dicho criterio, el estudio experimental sobre los motores diésel convencionales ha aportado los resultados mostrados en la figura (6.21), en donde se aprecia que en más 
del $80 \%$ de los casos la diferencia entre el ángulo de la dFQLmax obtenido mediante el análisis temporal de la señal de $d p$ y el obtenido mediante el cálculo de la tasa de liberación de calor, se encuentra en un rango de 0 a $2^{\circ}$.

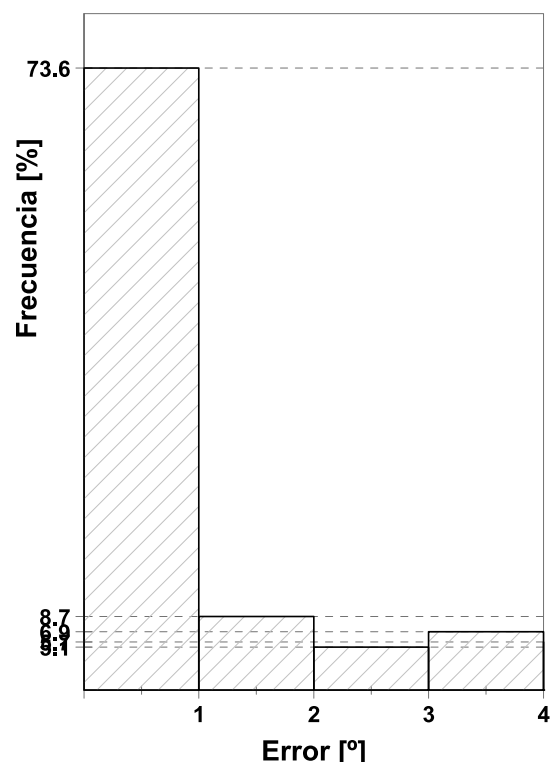

Figura 6.21: Histograma de la diferencia entre el ángulo de dFQLmax calculado y estimado. 


\subsubsection{Implementación en tiempo real.}

Con el objetivo de ilustrar la autenticidad de la metodología de diagnóstico de parámetros de la combustión mediante análisis en el dominio tiempo de la señal de la derivada de presión en cámara, propuesta y validada en un amplio rango de motores, se presentan las figuras 6.22, 6.23y 6.24 de un ensayo sobre el motor DW12 tomado como ejemplo para evaluar el potencial de dicha metodología.

Los resultados han sido comparados con los obtenidos mediante CALMEC y se muestra en las mismas figuras los datos estadísticos de las diferencias entre resultados.

El ensayo presenta una variación escalonada de la carga del motor (de 5 a $35 \%$ ) a régimen constante $(1500 \mathrm{rpm})$.

A partir de dichas figuras se puede concluir, refiriéndose a la metodología propuesta, que:

- La detección del SoC principal es muy minuciosa.

- La detección del SoC presenta mayores incertidumbres a medida que el SoC se aleja del punto muerto superior.

- La detección del EoC y del ángulo de la dFQLmax es fiable.

- Todos los parámetros estadísticos obtenidos entran en los márgenes obtenidos en la validación de la metodología para los diferentes motores ensayados en los apartados anteriores. 

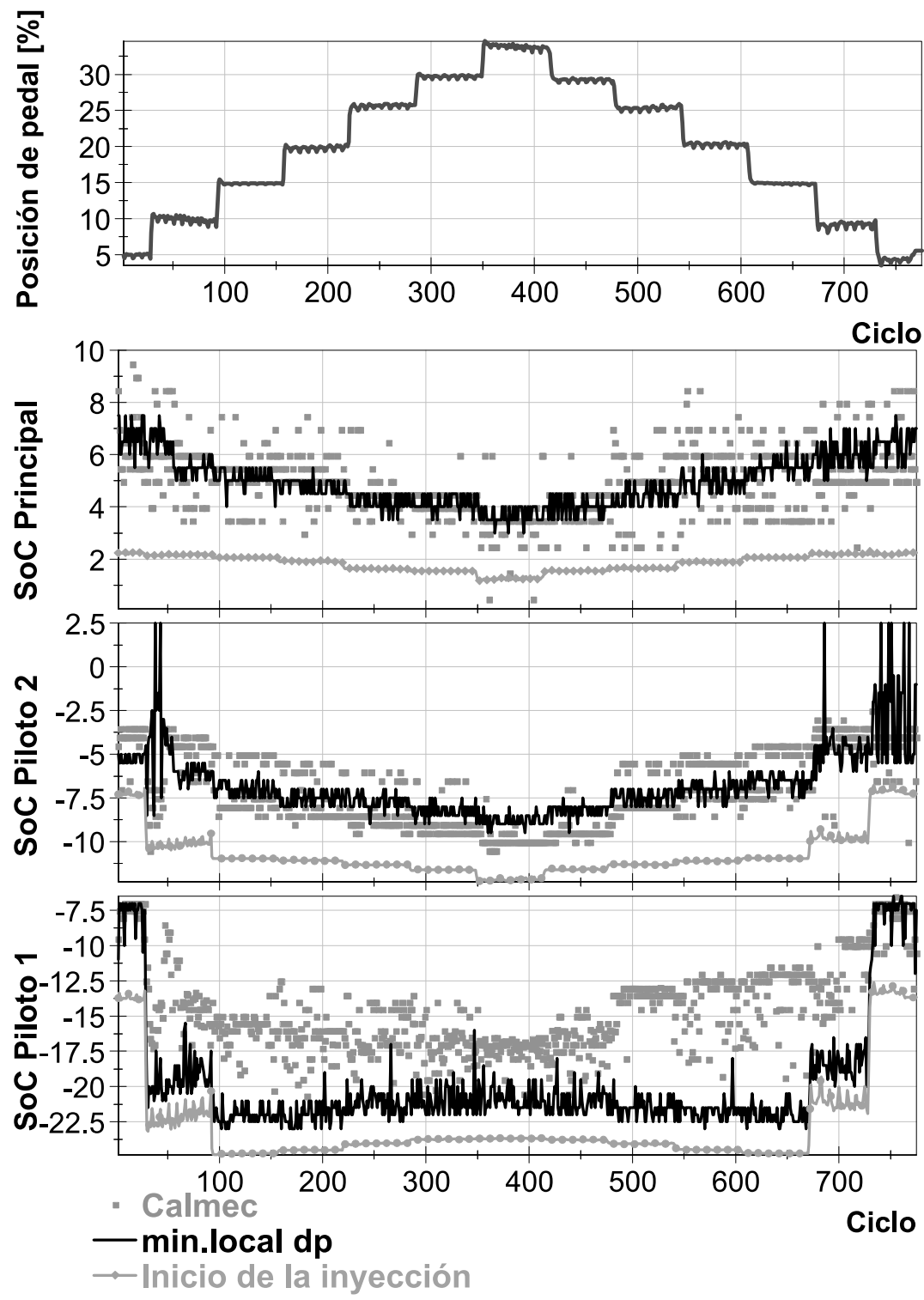

Figura 6.22: Detección del SoC en tiempo real motor DW12 a 1500 rpm y carga variable. 

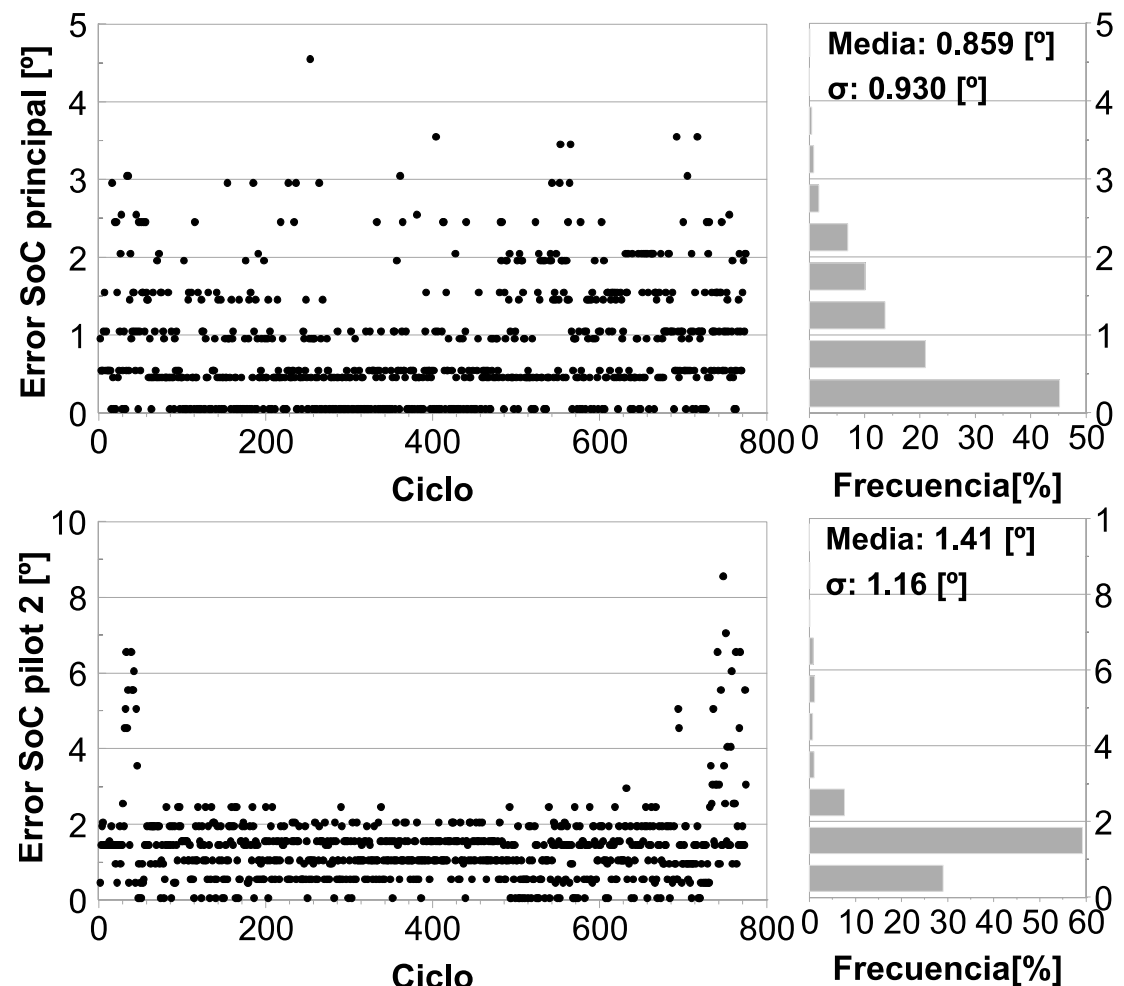

Frecuencia[\%]

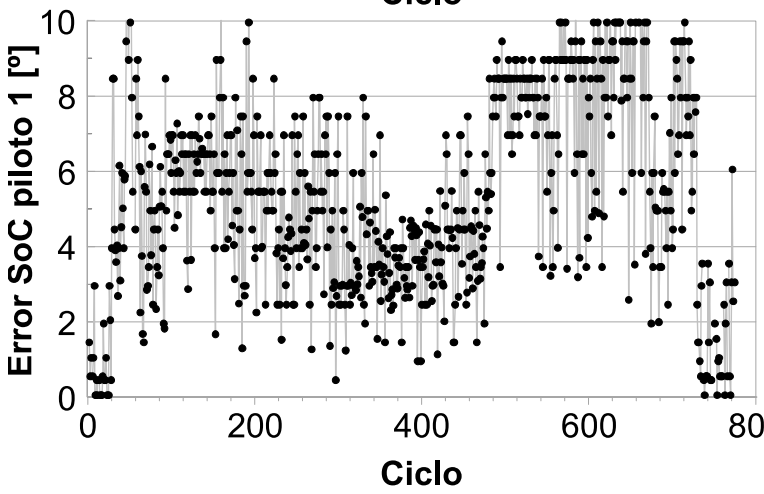

Media: $1.41\left[^{\circ}\right]$

$\sigma: 1.16\left[^{\circ}\right]$

$000102030405060^{0}$

Frecuencia[\%]

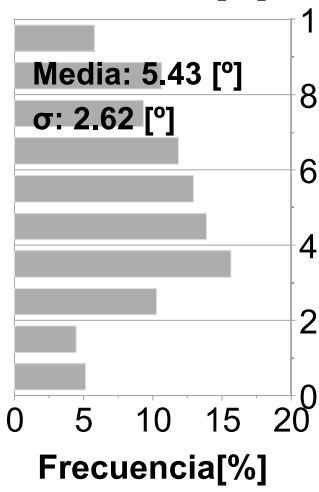

Figura 6.23: Distribución de la diferencia entre los resultados de CALMEC y los obtenidos mediante el tratamiento en el dominio tiempo de la señal de dp, para el caso de aplicación en tiempo real. 

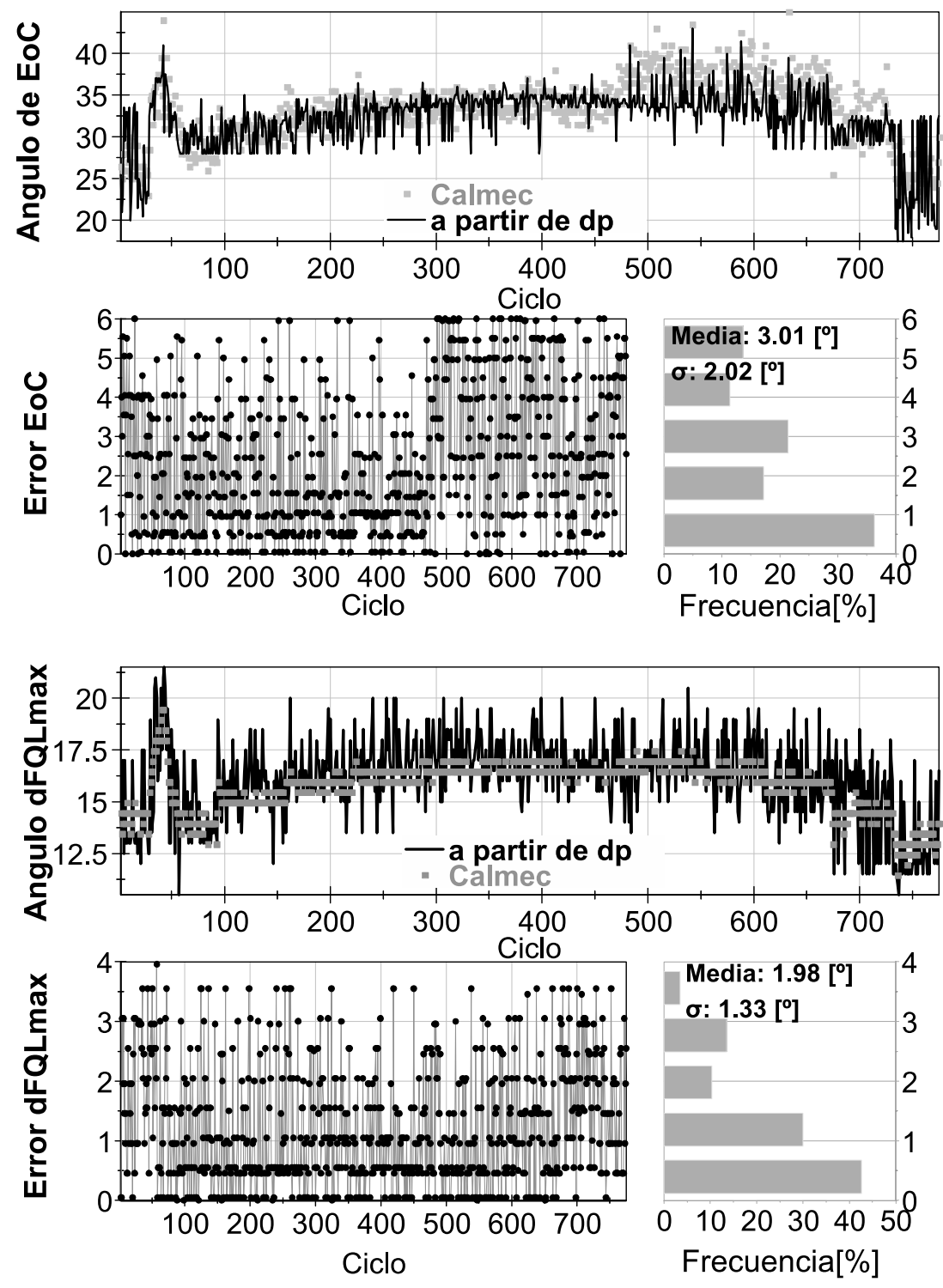

Figura 6.24: Detección del EoC y la dFQLmax y distribución de la diferencia entre los resultados de CALMEC y los obtenidos mediante el tratamiento en el dominio tiempo de la señal de dp, para el caso de aplicación en tiempo real. 


\subsection{Metodología de diagnóstico de la combustión a partir del análisis en dominio de frecuencias de la $d p$.}

\subsubsection{Herramientas de análisis conjuntas de la señal en tiempo y frecuencia}

La transformada rápida de Fourier (FFT) o su homóloga en dominio discreto (TDF), proporciona la herramienta principal para la representación frecuencial de las señales. Sin embargo, dicha herramienta sólo proporciona información sobre las frecuencias que componen la señal y no en qué instante temporal se han producido.

El análisis conjunto de tiempo-frecuencia, "Joint Time-Frequency Analysis" (JTFA), trata de localizar, en el dominio temporal, los cambios en las frecuencias que componen la señal. Es decir, a diferencia de las tecnologías convencionales de análisis de señales, el JTFA examina las señales en el dominio del tiempo y frecuencia simultáneamente.

El uso de este tipo de análisis es muy frecuente para el análisis de señales biomédicas, procesamiento de imágenes de radar, análisis de vibraciones de la máquina, pruebas y análisis de señales dinámicas. En cuanto al campo de motores, su uso sigue restringido a motores de encendido provocado, usando la señal de sensores de vibración, especialmente para diagnóstico de detonación o fallos de la combustión [3-6].

La base teórica del análisis tiempo-frecuencia [7-9] radica en un conjunto de técnicas que permiten la representación tridimensional de la señal en los ejes tiempo, frecuencia y amplitud simultáneamente. Así, para cada par (tiempo, frecuencia), le corresponde una amplitud que se le puede llamar "energía" del punto. Dicha amplitud es calculada mediante dos descomposiciones principales: lineales y cuadráticas. Mientras, con los algoritmos lineales se representa la descomposición de la señal en el plano tiempofrecuencia basándose en la amplitud de la señal temporal; mediante los algoritmos cuadráticos, la descomposición se realiza basándose en la energía de la señal proporcional al cuadrado del valor de la señal.

Los algoritmos lineales se basan en transformadas de Fourier de corta duración, "Short Time Fourier Transform" (STFT), la expansión de Gabor o la transformada Wavelet. Siendo el algoritmo basado en la STFT el más utilizado debido a su sencillez de implementación y su bajo coste computacional [9].

Para una señal de larga duración, se extraen ventanas de datos sucesivas obteniendo una sucesión de tramos de la señal. A cada tramo se le aplica la transformada de Fourier obteniendo los componentes en frecuencia de cada tramo. Así, se obtiene la evolución de los componentes frecuenciales de la señal a lo largo del tiempo.

Para una señal muestreada $(s[i])$, la STFT se define como:

$$
S[m, n]=\sum_{i=m \cdot \Delta M-L / 2}^{m \cdot \Delta M+L / 2} s[i] \cdot \zeta[i-m \cdot \Delta M] \cdot e^{-j \cdot 2 \cdot \pi \cdot i / N}
$$

Siendo:

$s \quad$ la señal muestreada, 
la amplitud de la señal en la representación tiempo-frecuencia para el instante de tiempo discreto o muestra $m$ y la frecuencia $n$

$\zeta$ la función de forma de la ventana de datos de ancho $L$ con desplazamiento entre tramos de $\Delta M$.

En efecto, la elección del tamaño de la ventana es crítica a la hora de obtener buena resolución. La resolución temporal de la STFT es proporcional a la duración efectiva de la ventana, y la resolución en frecuencia es proporcional al ancho de banda efectivo de la misma, por lo que, para conseguir buena resolución temporal será necesaria una ventana muy estrecha, mientras que para el caso de la frecuencia se necesitará una ventana ancha.

Los métodos cuadráticos como espectrogramas, distribución Choi-Williams y distribución de Wigner-Ville, se basan en la aplicación de transformaciones de segundo grado, es decir, elevando las amplitudes al cuadrado, dando así una representación de cómo el espectro de potencia de una señal evoluciona con el tiempo (espectrograma).

Detallar la matemática, las ventajas e inconvenientes de cada método se escapa del contexto de la presente tesis. Por lo tanto, la elección del método basado en la STFT y espectrogramas para el desarrollo de la presente tesis viene justificada por la sencillez y la facilidad de implementación de estos algoritmos.

Los espectrogramas representan una potente herramienta visual para interpretar el comportamiento de la señal a lo largo del tiempo. Por ejemplo, en las aplicaciones del JTFA en estudio de la combustión, Rizzoni y otros [3] usan un test de hipótesis para detectar la detonación o el knock en los motores de encendido provocado a partir de sensores de vibración. En el caso de Kim [6], se determina un rango de frecuencias que al estar visible en la representación tiempo-frecuencia hace que se acepte la hipótesis de la presencia del fenómeno en estudio.

Sin embargo, no es fácil automatizar dicho análisis y lograr de forma directa parámetros de fácil manejo que puedan ser relacionados con los fenómenos o los eventos que se pretenden estudiar. Esto es debido, principalmente, al gran tamaño de datos obtenidos por este tipo de análisis. Por lo tanto, la aplicación directa del JTFA no es eficiente para el campo de control y sólo puede ser ampliamente aprovechada para el análisis online de fenómenos como el de combustión.

Aun así, existe la posibilidad de compactar la información contenida en las representaciones tiempo-frecuencia. Para ello, se acude a parámetros como el de la frecuencia media instantánea (MIF) que da información del valor de la frecuencia dominante, en un determinado momento, dentro del campo de datos analizados y representados en el dominio tiempo-frecuencia. Dicha propiedad cumple con la ecuación:

$$
\operatorname{MIF}(m)=\frac{\int_{-\infty}^{+\infty} n \cdot S(m, n) \cdot \mathrm{d} n}{\int_{-\infty}^{+\infty} S(m, n) \cdot \mathrm{d} n}
$$

La frecuencia media instantánea es, todavía, un concepto más intuitivo que el exactamente definido en la ecuación (6.4.2). De hecho, el uso de todo el espectro de la ventana de la señal (instantes pasados y futuros) para calcular la MIF en un instante $(m)$ dado, la aparición de valores negativos o el efecto de los términos cruzados en el cálculo 
de la potencia de la señal requerido para el cálculo de la MIF, son paradojas difíciles de asimilar si se interpreta la MIF en su sentido literal. Cohen [7] señala, citando varios trabajos, que la descripción matemática exacta y la comprensión del concepto de cambio de frecuencia está lejos de ser evidente y puede afirmarse que no es una cuestión resuelta. Por lo tanto, el uso de este parámetro no tiene que ser interpretado en el sentido literal de su definición, sino como una magnitud que puede relacionarse con las variaciones temporales de las frecuencias que componen la señal en estudio y cuya variación tiene que ser interpretada por criterios del investigador.

En la presente tesis, se parte de la hipótesis de que al producirse la combustión, debe aparecer en la señal de presión en cámara nuevas frecuencias de mayor magnitud que frecuencias existentes sin combustión, dichas frecuencias pueden ser causadas por la propia combustión o por efectos de los elevados gradientes de presión y altas temperaturas sobre el elemento sensor tal como la aparición de resonancias. No se pretende ahora evaluar con exactitud dichas frecuencias o su origen real, sino más bien se quiere demostrar de forma experimental, que variaciones importantes en la MIF pueden relacionarse con los parámetros de la combustión (SoC, EoC, etc).

\subsubsection{Aplicación de los métodos basados en el análisis en frecuencias de la dp.}

Los métodos basados en el análisis en frecuencia pueden ser muy útiles para el diagnóstico de la combustión online, aunque debido a su coste computacional relativamente alto, no son los más adecuados para el diagnóstico en tiempo real o al menos requieren sistemas lo suficiente potentes para asegurar un tiempo de cómputo fijo.

El uso más extendido de la TDF en el diagnóstico de la combustión, es el del filtrado (5.3). Incluso en los estudios de resonancias y ruidos de combustión, se usa esta herramienta principalmente para filtros digitales. Pese al aporte de algunos trabajos de investigación para sacar mayor aprovecho de esta herramienta (aporte a la estimación del dosado [10], detección y corrección de fallos de inyección [11], etc.) su uso sigue siendo limitado.

Con objeto de mostrar nuevas herramientas para el estudio de la combustión, se exponen en este apartado herramientas basadas en el análisis en frecuencia de la $d p$ para estimar parámetros de la combustión.

Para la obtención de la ley de liberación de calor, se puede emplear la TDF directamente a la señal de la derivada de la presión, implementando algunos criterios matemáticos relacionados con dicha transformada como se muestra a continuación:

Partiendo de la función de la tasa de liberación de calor aparente (ecuación (3.2.1)), la TDF de dicha función se puede escribir, tomando en cuenta el coeficiente isoentrópico $\gamma$ constante y la propiedad de linealidad de la TDF, como:

$$
\mathscr{F}\{d F Q L\}(\xi)=\frac{\gamma}{\gamma-1} \cdot \mathscr{F}\left\{p \cdot \frac{d V}{d \alpha}\right\}(\xi)+\frac{1}{\gamma-1} \cdot \mathscr{F}\left\{V \cdot \frac{d p}{d \alpha}\right\}(\xi)
$$

El teorema de la convolución afirma que la transformada de Fourier del producto 
de funciones es equivalente a la convolución de las transformadas de Fourier de dichas funciones $(\mathscr{F}\{f \cdot g\}=\mathscr{F}\{f\} * \mathscr{F}\{g\})$ siendo la convolución, denotada como $f * g$, la integral del producto de ambas funciones después de desplazar una de ellas una distancia.

Para funciones continuas:

$$
(f * g)(x)=\frac{1}{\sqrt{2 \pi}} \int_{-\infty}^{+\infty} f(y) \cdot g(x-y) d y
$$

Y para el caso discreto:

$$
f[n] * g[n]=\sum_{-\infty}^{+\infty}\{f[m] \cdot g[n-m]\}
$$

Aplicando dicho teorema a la ecuación (6.4.3), se obtiene:

$$
\mathscr{F}\{d F Q L\}(\xi)=\frac{\gamma}{\gamma-1} \cdot\left(\mathscr{F}\{p\} * \mathscr{F}\left\{\frac{d V}{d \alpha}\right\}\right)+\frac{1}{\gamma-1} \cdot\left(\mathscr{F}\{V\} * \mathscr{F}\left\{\frac{d p}{d \alpha}\right\}\right)
$$

Además, considerando la propiedad de la transformada de la derivada :

$$
\mathscr{F}\{d F Q L\}(\xi)=\frac{\gamma}{\gamma-1} \cdot(\mathscr{F}\{p\} * j \cdot \xi \cdot \mathscr{F}\{V\})+\frac{1}{\gamma-1} \cdot(\mathscr{F}\{V\} * j \cdot \xi \cdot \mathscr{F}\{p\})
$$

Donde $\xi$ representa los armónicos en radianes.

Por lo tanto, el cálculo de la transformada de Fourier de la $d F Q L$ requiere el vector de los armónicos, que es solo función del número de muestras por ciclo, y las transformadas de Fourier del volumen y la de la presión o en su caso la de la derivada de la presión. En caso de medida directa de la derivada de la presión, se usará la propiedad de la transformada de Fourier para la integral de funciones dada por definición por:

$$
\mathscr{F}\{p\}(\xi)=\frac{1}{j \cdot \xi} \cdot \mathscr{F}\{d p\}(\xi)+\pi \cdot \mathscr{F}\{d p\}(0) \cdot \delta(\xi)
$$

Siendo $\delta(\xi)$ la función delta de Dirac.

Si la matemática que conlleva la ecuación (6.4.5) puede parecer complicada, su implementación es muy sencilla y permite mejorar considerablemente el tiempo computacional y los requisitos de memoria. Además, se puede conseguir reducir aún más el tiempo computacional trabajando con transformadas bidimensionales y/o reduciendo los tamaños de la transformada de Fourier del volumen sabiendo que este último se compone básicamente de una función coseno y otra senoïdal tal como indica la ecuación (4.3.1) analizada en el capítulo 4:

$$
V=V_{c c}+\frac{\pi \cdot D^{2}}{4}\left[\left(L_{b}+S / 2\right)-S / 2 \cdot \underline{(\cos (\alpha)}+\sqrt{\left.1 / \lambda^{2}-\underline{\operatorname{sen}^{2}(\alpha)}\right)}\right]+\Delta V_{p}+\Delta V_{i}
$$


Sin animo a ser exhaustivo en el análisis matemático que puede aportar el cálculo de la tasa de liberación de calor mediante transformada de Fourier, se plantea la siguiente metodología para obtener información de la combustión:

1. Se calcula la transformada de Fourier del volumen.

2. Se construye el vector de los armónicos $\xi$ multiplicado por el número complejo $j$.

3. Se calcula la transformada de la señal de presión. Implementando directamente el filtrado, es decir, multiplicando los elementos de la transformada de la señal medida por el filtro deseado.

4. Se calcula el primer sumatorio $\mathscr{F}\{p\} * j \cdot \xi \cdot \mathscr{F}\{V\}$ de la ecuación (6.4.5). En este punto, dado que el primer armónico de esta operación coincide con el primer armónico de la $\mathscr{F}\{p \cdot d V\}$, su magnitud corresponde al valor medio de $p \cdot d V$, por lo tanto, multiplicando por el número de muestras, se obtiene el sumatorio de los elementos de $p \cdot d V$ y el cálculo de la presión media indicada es instantáneo multiplicando dicha suma por el incremento angular y dividiendo el resultado por el volumen desplazado.

5. Se calcula el segundo sumatorio $\mathscr{F}\{V\} * j \cdot \xi \cdot \mathscr{F}\{p\}$ de la ecuación (6.4.5) y se completa el cálculo de dicha fórmula.

6. Una vez obtenida la transformada de la dFQL, se maniobra del mismo modo utilizado para el cálculo de la presión media indicada para calcular el calor total liberado durante el ciclo $\left(\Delta \alpha \cdot \sum_{N} d F Q L(\alpha)\right)$ partiendo de $\mathscr{F}\{d F Q L\}(0)=\frac{1}{N}$. $\sum_{N} d F Q L(\alpha)$.

7. Mediante la transformada inversa de Fourier se puede obtener la tasa de liberación de calor instantánea, Sin embargo, es de mayor utilidad obtener la propia ley de liberación de calor utilizando la propiedad de la integral de la transformada de Fourier antes de proceder al cálculo de la transformada inversa.

$$
\mathscr{F}\{F Q L\}(\xi)=\frac{1}{j \cdot \xi} \cdot \mathscr{F}\{d F Q L\}(\xi)+\pi \cdot \mathscr{F}\{d F Q L\}(0) \cdot \delta(\xi)
$$

8. Y finalmente, conocida la cantidad de calor librada durante el ciclo, se puede localizar los ángulos correspondientes a los parámetros de interés para el diagnóstico de la combustión.

La desventaja principal es el tiempo de cálculo, casi 5 veces mayor que el tiempo de cálculo necesario para calcular la $d F Q L$ aparente, mientras que las principales ventajas de esta metodología son:

- Disponibilidad de mayor información de los componentes que afectan el cálculo de la liberación de calor en dominio frecuencia. 
- Eliminación de incertidumbres y desfases de las señales debidas a la derivada numérica de la presión y del volumen.

- Obtención de parámetros de la combustión sin necesidad de realizar cálculos extras.

- Y principalmente, la facilidad de implementación de filtros, ya que no solo se puede filtrar la señal de presión, sino que también es posible obtener tasas de liberación de calor más fieles a las teóricas.

En efecto, considerando el resultado de varias investigaciones donde se modela la $d F Q L$ mediante funciones [12-15], se puede partir de la transformada de Fourier del modelo para obtener un filtro óptimo que minimice el error entre el modelo y la $d F Q L$ calculada a partir de las señales medidas.

Existen en la bibliografía de tratamiento de señales [9,16-18], infinidad de filtros que sirvan para el propósito anterior. Entre ellos, se puede señalar los filtros de Kalman, los adaptados, llamados también detectores de ajuste "Matched filter" o "North filter", y los filtros basados en el estimador de Wiener donde se define el filtro optimo como el cociente entre el espectro del modelo y el espectro de potencia de la señal calculada [19].

A modo de ejemplo, se puede usar el espectro de potencia de una combinación de funciones de Wiebe para definir la ley de combustión teórica, como las planteadas por Galindo y otros [15], para obtener el filtro óptimo a aplicar a la transformada de Fourier de la $d F Q L$ experimental.

Otro ejemplo sería usar modelos empíricos de la combustión, más complejos, como el modelo ACT planteado en el trabajo de Fenollosa [14]. Par ello, se aplica el filtro $H$, calculado mediante ecuación (6.4.6), a la TDF de la $d F Q L$ aparente, calculada como se ha planteado anteriormente.

$$
H(\xi)=\frac{\mathscr{F}^{2}\left(d F Q L_{A C T}\right)}{\mathscr{F}^{2}\left(d F Q L_{A C T}\right)+\mathscr{F}^{2}\left(d F Q L_{\text {aparente }}\right)}
$$

Donde:

$$
\begin{aligned}
& \mathscr{F}^{2}\left(d F Q L_{A C T}\right) \text { representa el espectro de la dFQL teórica calculada median- } \\
& \text { te el modelo ACT [14]. } \\
& \mathscr{F}^{2}\left(d F Q L_{\text {aparente }}\right) \text { representa el espectro de la dFQL aparente calculada } \\
& \text { mediante el análisis en frecuencias de la } d p \text {. }
\end{aligned}
$$

Dicho filtro $H$ es una variedad del estimador de Wiener que minimiza el error entre la $d F Q L$ teórica, calculada mediante ACT ( $\left.d F Q L \_A C T\right)$, y la $d F Q L$ aparente. La figura (6.25) representa casos de aplicación de esta metodología. Donde, $d F Q L \_$Fourier es obtenida mediante la transformada inversa de $\mathscr{F}\left\{d F Q L_{\text {Fourier }}\right\}$ ( $\xi$ ) calculada mediante el producto de las ecuaciones 6.4 .5 y 6.4 .5 es decir:

$$
\mathscr{F}\left\{d F Q L_{\text {Fourier }}\right\}(\xi)=H(\xi) \cdot \mathscr{F}\left\{d F Q L_{\text {aparente }}\right\}(\xi)
$$


La figura (6.25) muestra como, independientemente del punto de funcionamiento del motor, la $d F Q L \_$Fourier obtenida presenta un intermedio entre la aparente ( $d F Q L \_$aparente) y la teórica ( $\left.d F Q L \_A C T\right)$. Además, comparando con la $d F Q L$ obtenida mediante $C A L M E C$, se confirma que el resultado es válido.

En los casos presentados, el filtro propuesto no permite obtener resultados más semejantes a la $d F Q L$ teórica debido a que la $d F Q L$ aparente aunque comparte componentes en frecuencia con la $d F Q L$ teórica, sus amplitudes son bien distintas. El filtro atenúa las frecuencias no compartidas pero no las elimina ya que tienen una amplitud significativa en la $d F Q L$ aparente.

Así pues, el método minimiza el error entre el modelo teórico y el calculado, pero no asegura que el resultado sea el óptimo. También mejora significativamente la $d F Q L$ aparente, pero no se logra la óptima de $C A L M E C$. Eso se refleja en las leyes de liberación de calor donde, en todo caso, se obtiene una $F Q L$ intermedia entre la $F Q L$ teórica ( $F Q L \_$ACT) y la $F Q L$ óptima ( $\left.F Q L \_C A L M E C\right)$ como se presenta en la figura (6.25) para los puntos de funcionamiento del motor DW12 reflejados en la tabla (6.4).

\begin{tabular}{cccc} 
Caso & Régimen(rpm) & Par(Nm) & PMI(bar) \\
\hline \hline A & 1000 & 64.4 & 5 \\
B & 2000 & 221.5 & 15 \\
C & 3000 & 1 & 3 \\
D & 4000 & 226.7 & 17.5 \\
\hline
\end{tabular}

Tabla 6.4: Puntos de funcionamiento del motor DW12 para los datos de la figura(6.25). 

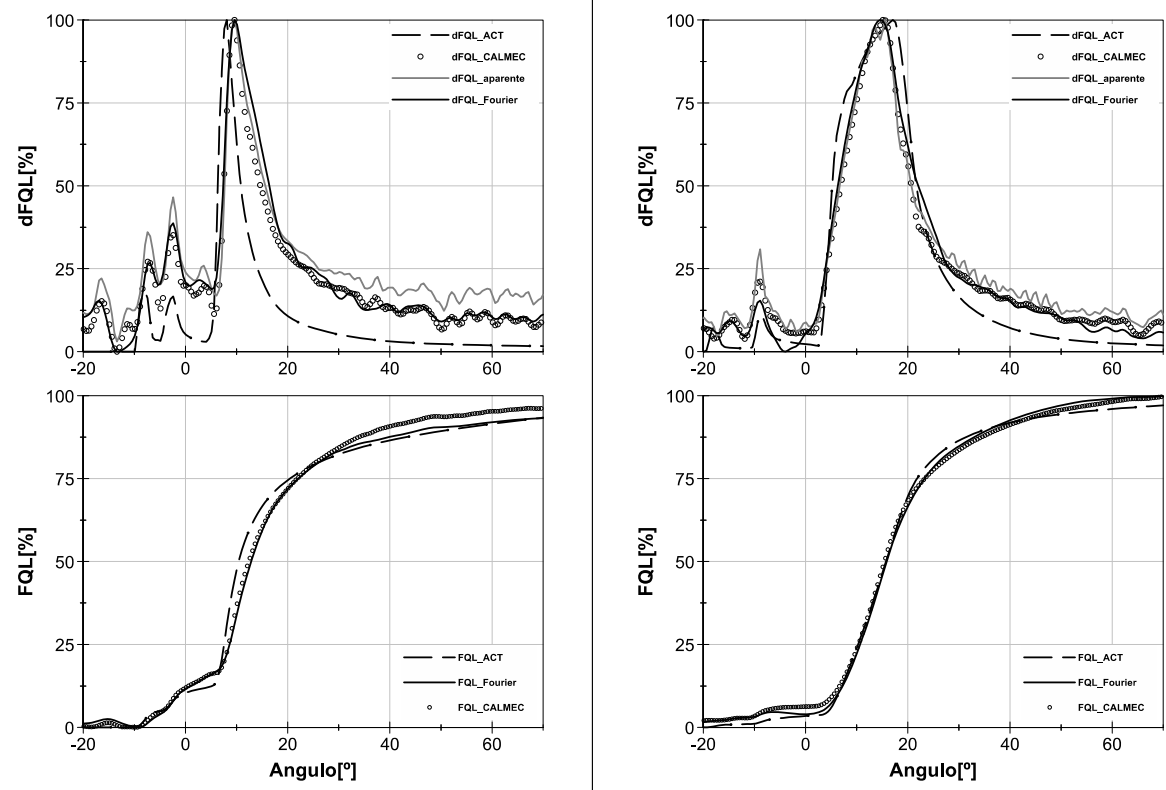

Caso A

Caso B
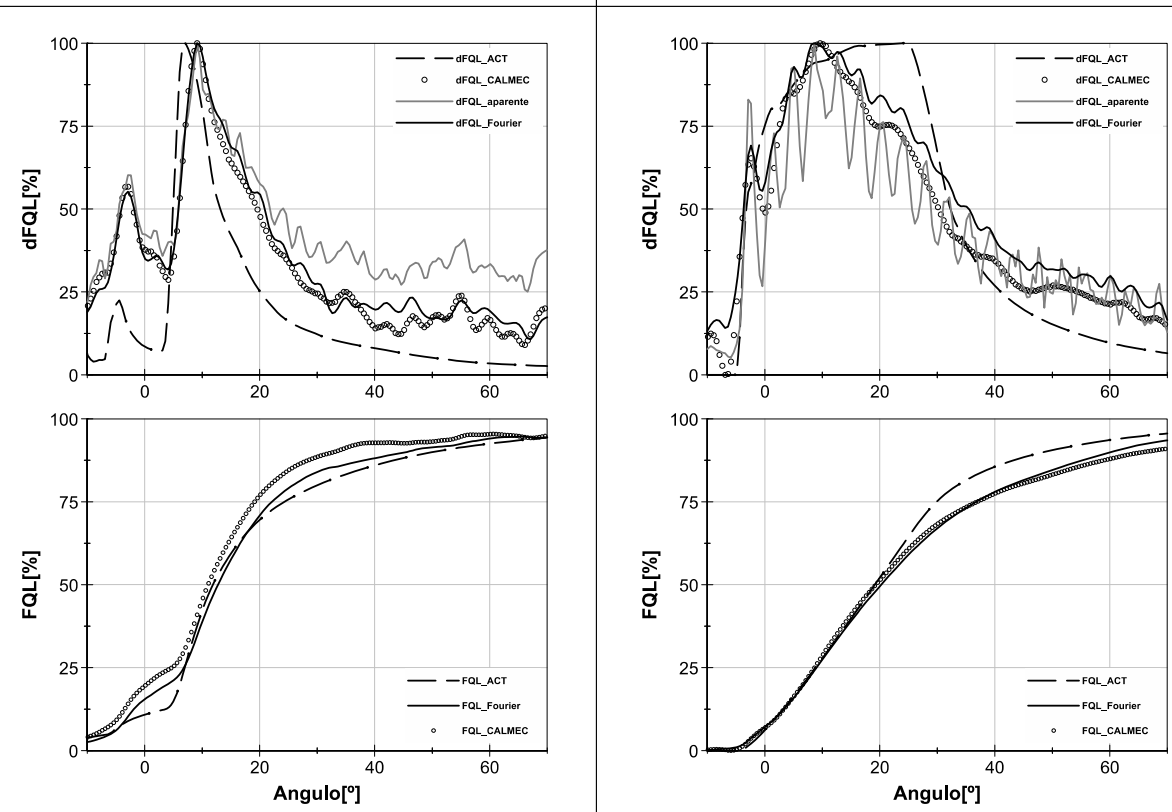

Caso C

Caso D

Figura 6.25: Leyes de liberación de calor mediante transformada de Fourier y filtrado en dominio de frecuencias. 
En definitiva, el estudio de la combustión en el dominio frecuencia sigue siendo un campo poco explorado. Algunos métodos y conceptos de tratamiento de señales, como el de banco de filtros, pueden ser de gran utilidad para mayores ajustes del análisis de la combustión en el campo de la investigación. Otros, pueden tener aplicaciones más directas para el diagnóstico de la combustión online como es el caso del análisis TiempoFrecuencia de la señal de la derivada de presión en cámara.

\subsubsection{Aplicación de los métodos basados en el análisis conjunto Tiempo- Frecuencias de la $d p$.}

En el presente trabajo, se han empleado métodos de análisis cuadrático TiempoFrecuencia que permiten, utilizando la transformada de Fourier, crear el espectrograma de la señal que representa la energía contenida en la señal en función del tiempo y la frecuencia.

El algoritmo adoptado, como se comentó anteriormente (6.4.1), se basa en la transformada rápida de Fourier STFT, llamada también transformada de Fourier por ventanas "the windowed Fourier transform" o transformada de Fourier deslizante "the sliding Fourier transform", donde se divide la señal en ventanas temporales a las que se les aplica la transformada de Fourier.

En los instantes en los que se produce la combustión, se aprecian sobre-oscilaciones de gran magnitud en la derivada de la presión en cámara, lo que se traduce en la aparición de una energía significativa en la señal.

La figura (6.26) representa para la señal $d p$ del motor en arrastre (gráfica superior) el espectrograma donde se ilustra que la señal no contiene energía importante en sus componentes de frecuencias considerables. La energía de la señal a frecuencias bajas es similar a la energía media de la señal que se puede calcular mediante la ecuación (6.4.7).

$$
\text { Energiamedia }_{d p}(\alpha)=\sqrt{\frac{1}{N} \sum_{\alpha-N / 2}^{\alpha+N / 2} d p^{2}(\alpha)}
$$

Puesto que la señal de $d p$ en cualquier punto de funcionamiento del motor es la suma de: la $d p$ en arrastre, el gradiente de presiones debido a la combustión y el ruido. La información presente en el espectrograma de la señal de $d p$ del motor en arrastre va a estar presente en todos los puntos de funcionamiento del motor. 


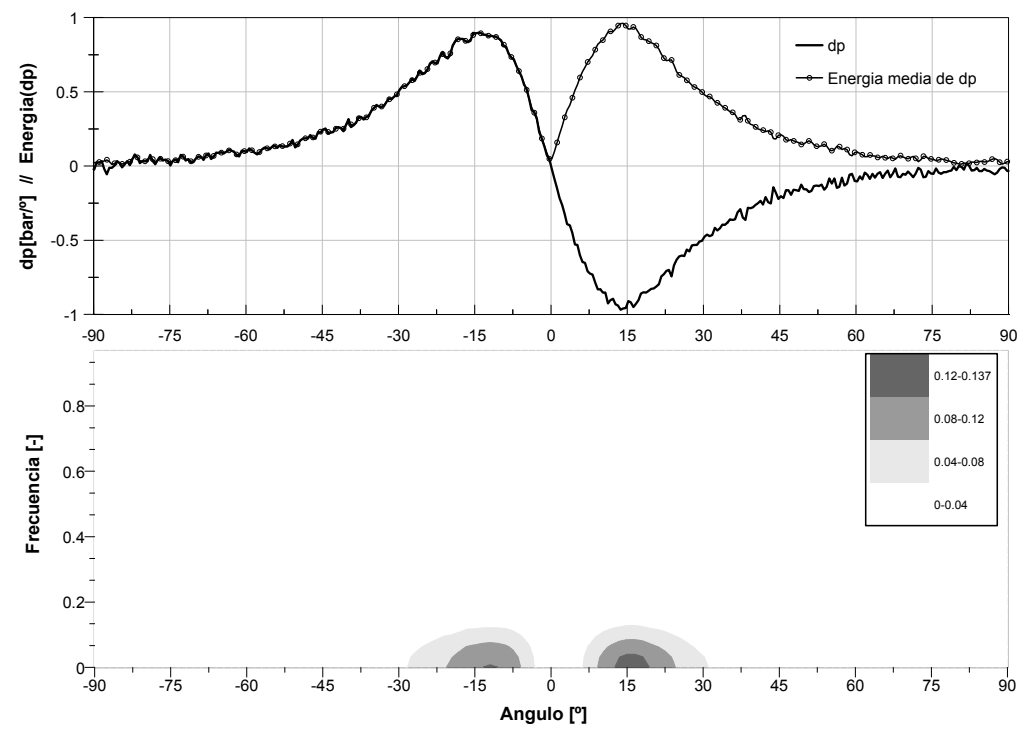

Figura 6.26: Análisis conjunto de tiempo-frecuencia de la señal de la derivada de presión (Motor M9R en arrastre).
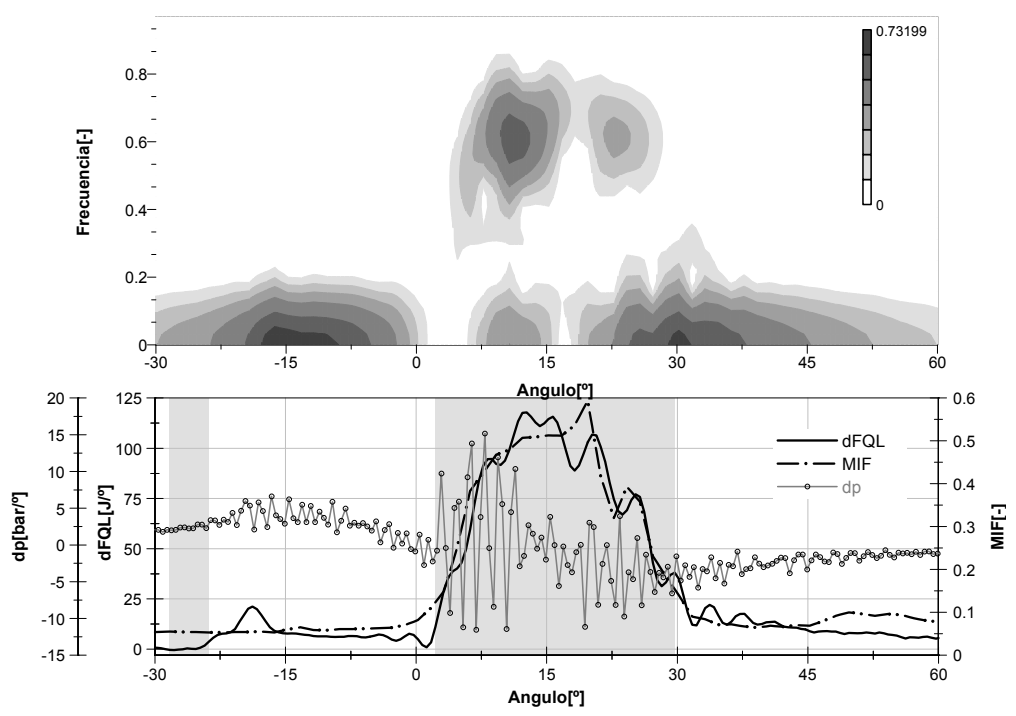

Figura 6.27: Análisis conjunto de tiempo-frecuencia de la señal de la derivada de presión (Motor DW10 en combustión). 
La figura (6.27) representa, en la parte superior, el espectrograma de la $d p$ para un punto de funcionamiento en combustión. Se diferencia en la zona de frecuencias altas, una energía considerable debida a los cambios bruscos en la $d p$ ocasionados por los fenómenos de la combustión.

En la misma figura, se representa (en la gráfica inferior) la señal $d p$ original junto con la tasa de liberación de calor y la frecuencia media instantánea para un punto de funcionamiento del motor con dos inyecciones (presentadas por la zona sombreada). Se aprecia como al aumentar la tasa de liberación de calor, la frecuencia media instantánea de la $d p$ crece. Eso permite detectar el inicio de la combustión tomando como criterio el ángulo donde la frecuencia media instantánea se haga creciente.

Del mismo modo, el final de la combustión puede ser localizado bajo el criterio de que la frecuencia media de la señal de $d p$ vuelve a tomar los valores iniciales.

Las discontinuidades presentes en la combustión alteran la forma de la frecuencia media instantánea provocando picos que no deberían relacionarse con máximos locales de la tasa de liberación de calor. Aun así, generalmente, el máximo de la frecuencia media instantánea suele coincidir con la $d F Q L$ máxima mientras, en el ángulo correspondiente, la energía de la señal presentada en el espectrograma es considerable.

La metodología del análisis conjunto de tiempo-frecuencia de la señal de la $d p$ es fiable para combustiones fuertes, lo que hace de ella una herramienta robusta para detección de fallos de la combustión y/o knock.

Por otra parte, las combustiones piloto, suelen aportar frecuencias muy bajas a la señal, complicando su detección en la frecuencia media instantánea.

Aplicando los criterios de la detección de la combustión principal a partir del análisis conjunto tiempo-frecuencia se obtienen los resultados de la figura (6.28) donde se muestran algunos resultados de la comparativa entre la metodología propuesta y los resultados obtenidos mediante $C A L M E C$. En la misma figura se representa el histograma de la distribución del error cometido para más de los 324 puntos de funcionamiento de motores diésel convencionales (DW10, DW12 y M9R).

La tabla (6.5) muestra los parámetros estadísticos finales del error en la detección de algunos parámetros de la combustión, mediante la frecuencia media instantánea de la señal de la $d p$. A partir de dicha tabla, se puede concluir que la MIF, aun teniendo un coste computacional importante, aporta unos resultados satisfactorios.

\begin{tabular}{|c|c|c|c|}
\hline & Media $\left[{ }^{\circ}\right]$ & Desviación Típica [ $\left.{ }^{\circ}\right]$ & Máximo [ [] \\
\hline SoC principal & 0.87 & 0.8 & 4 \\
\hline dFQLmax & 1.49 & 1.08 & 5.5 \\
\hline EoC & 3.28 & 2.16 & 15.5 \\
\hline
\end{tabular}

Tabla 6.5: Parámetros estadísticos del error en la detección de algunos parámetros de la combustión mediante la frecuencia media instantánea de la señal de la dp. 

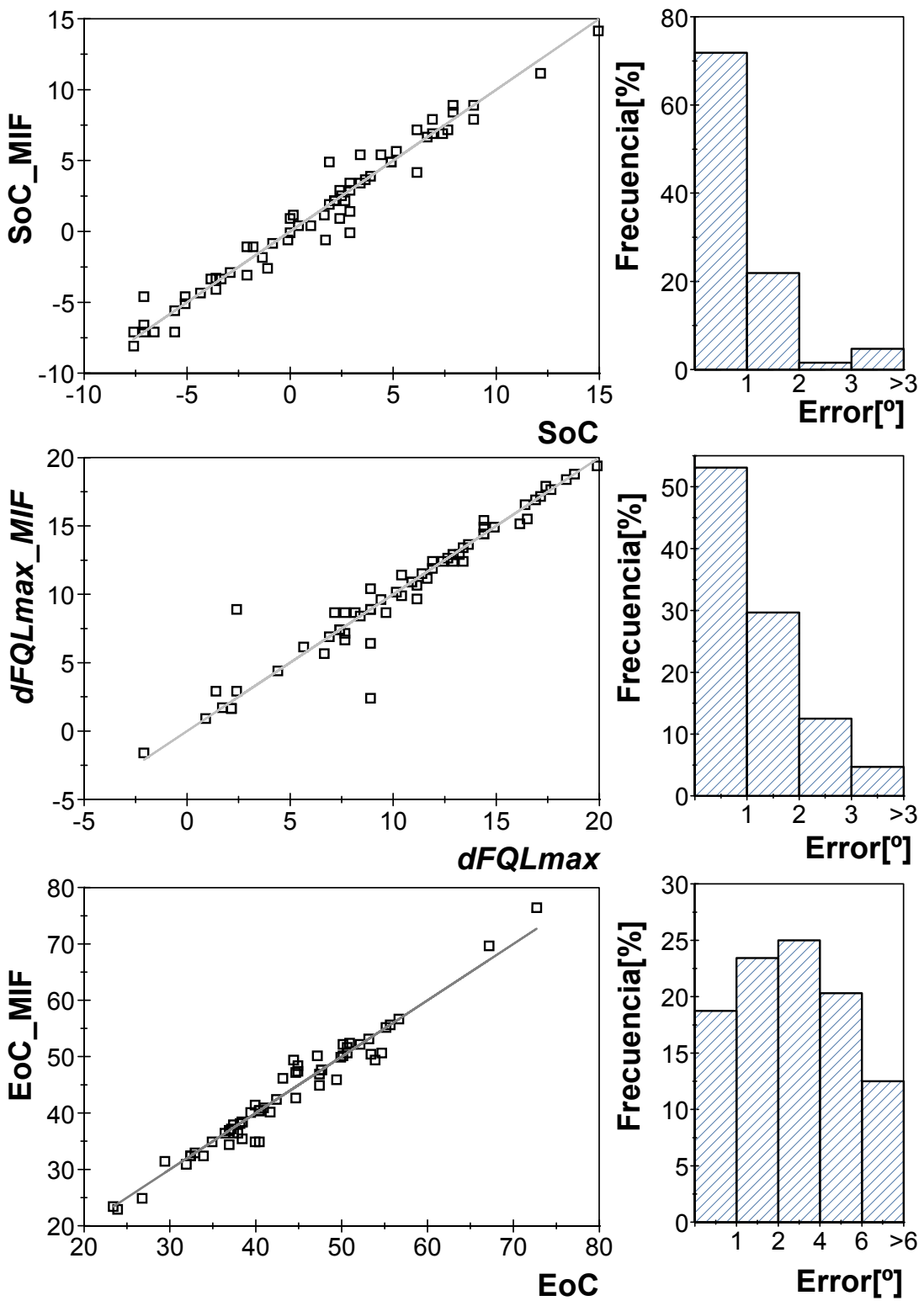

Figura 6.28: Validación de los resultados del análisis conjunto de tiempo-frecuencia de la señal de la $d p$. 


\subsection{Análisis comparativo de las metodologías propuestas y conclusiones.}

En los apartados anteriores se han presentado nuevas metodologías para el diagnóstico de la combustión basadas en la $d p$, se han mostrado los resultados y se han discutido las particularidades de cada metodología.

Llegados a este punto, la tabla (6.6) presenta un resumen de las prestaciones de cada metodología, permitiendo así un análisis comparativo entre las diferentes metodologías.

\begin{tabular}{|c|c|c|c|c|c|c|}
\hline $\begin{array}{l}\text { Análisis de dp en } \\
\text { dominio temporal }\end{array}$ & $\uparrow \uparrow \uparrow$ & $\downarrow$ & $\downarrow$ & $\leftrightarrow$ & $\sqrt{ }$ & $\sqrt{ } \sqrt{ }$ \\
\hline $\begin{array}{c}\text { Análisis conjunto } \\
\text { tiempo-frecuencia } \\
\text { de dp }\end{array}$ & $\uparrow \uparrow$ & $\uparrow \uparrow$ & $\uparrow \uparrow$ & $\uparrow$ & $\sqrt{ }$ & -- \\
\hline $\begin{array}{l}\text { Análisis de dp en } \\
\text { dominio frecuencia }\end{array}$ & $\uparrow \uparrow \uparrow$ & $\uparrow$ & $\uparrow \uparrow$ & $\uparrow \uparrow \uparrow$ & $\sqrt{ }$ & $\sqrt{ }$ \\
\hline $\begin{array}{l}\text { Ley de liberación } \\
\text { de calor aparente }\end{array}$ & $\uparrow$ & $\uparrow$ & $\uparrow \uparrow$ & $\uparrow \uparrow$ & $\checkmark$ & $\sqrt{ }$ \\
\hline CALMEC & $\uparrow \uparrow \uparrow \uparrow \uparrow$ & $\uparrow \uparrow \uparrow \uparrow \uparrow$ & $\uparrow \uparrow \uparrow \uparrow \uparrow$ & $\uparrow \uparrow \uparrow \uparrow \uparrow$ & $\otimes$ & $\otimes$ \\
\hline
\end{tabular}

Tabla 6.6: Resumen de las prestaciones de las metodologías de diagnóstico de la combustión tratadas.

El análisis de la combustión detallado mediante $C A L M E C$ es especialmente recomendado para diagnóstico offline y temas de investigación. Aporta información completa y precisa del proceso de la combustión, a costa de requerir un número elevado de variables medidas y un coste computacional muy elevado. Esto impide el uso de este tipo de herramientas para diagnóstico online y no es eficiente su uso para aportar información a la ECU. Sin embargo tomándolo como referencia para validación de los resultados de cada una de las metodologías propuestas, se puede concluir lo siguiente : 
1. En cuanto al diagnóstico de la combustión a partir del análisis de $d p$ en dominio temporal:

- Tiene un coste computacional mínimo.

- Solo requiere el sensor de la presión en cámara y parámetros de la inyección.

- Los resultados confirman que su precisión en la detección del SoC esta relacionada con la magnitud de la combustión (precisión excelente para las combustiones principales pero hay mayores incertidumbres para combustiones piloto débiles).

- Permite obtener, con muy buena precisión, la estimación de parámetros como el ángulo de presión máxima, el ángulo de dpmax y el ángulo de la dFQLmax.Y pese a las incertidumbres en los criterios de la detección del EoC, los resultados son satisfactorios.

Lo anterior hace del diagnóstico de la combustión a partir del análisis de $d p$ en dominio temporal el más recomendado para diagnóstico y control en tiempo real. Por lo tanto, se abren muchas posibilidades para su implementación en las ECUs de forma directa o mediante interfaces electrónicos intermedios.

2. En relación al diagnóstico de la combustión a partir del análisis conjunto tiempofrecuencia de $d p$ :

- Tiene la importante ventaja de la fácil interpretación del interfaz gráfico que presenta los espectrogramas.

- El uso de la frecuencia instantánea media aporta resultados satisfactorios. Dichos resultados, si son comparados con los obtenidos con la metodología propuesta para el diagnóstico de la combustión a partir del análisis de $d p$ en dominio temporal, tal y como se muestra en la tabla (6.7), revelan una mejora en la detección del EoC.

\section{SoC principal \\ dFQLmax}

$\begin{array}{llll}\text { Análisis de dp en dominio } & 0.5 \pm 1.07^{\circ} & 4.3 \pm 4.72^{\circ} & 0.5 \pm 0.7^{\circ} \\ \text { temporal }\end{array}$

\begin{tabular}{llll}
$\begin{array}{l}\text { Análisis conjunto } \\
\text { tiempo-frecuencia de dp }\end{array}$ & $0.87 \pm 0.8^{\circ}$ & $3.28 \pm 2.16^{\circ}$ & $1.49 \pm 1.08^{\circ}$ \\
\hline
\end{tabular}

Tabla 6.7: Comparativa del error entre dos métodos propuestos.

- Requiere un coste computacional considerable y ajustes de parámetros (longitud y resolución de las ventanas) de análisis costoso. 
3. En la tabla (6.7) se comparan las medias del error cometido, por cada metodología, en el diagnóstico de los parámetros de interés: SoC, EoC y dFQLmax para las combustiones principales. Se aprecia que el método del análisis conjunto tiempofrecuencia de $d p$ únicamente es mejor para la detección del EoC.

4. El análisis de la $d p$ en dominio frecuencia:

- Brinda una nueva visión para los estudios de procesos de la combustión.

- Su uso para cálculo de la ley de liberación de calor aporta resultados de precisión intermedia entre la ley de liberación de calor aparente y una herramienta de diagnóstico de combustión completa.

- Permite aprovechar fases intermedias de cálculo para obtener parámetros de la combustión.

- Está justificado el coste computacional que le acompaña por la cantidad de información que se puede extraer de este tipo de análisis.

- Su uso para el diagnóstico de la combustión online es viable.

- Puede aportar mucha información a la ECU si se implementa en interfaces electrónicos intermedios. 



\section{Bibliografía del capítulo}

[1] S. Leonhardt, N. Müller, y R. Isermann. "Methods for engine supervision and control based on cylinder pressure information". IEEE/ASME Transactions on Mechatronic, 4:235-245, 1999.

[2] J.M. Desantes, A.J. Torregrosa, y A. Broatch. "Wavelet transform applied to combustion noise analysis in high-speed di diesel engines". SAE paper, (2001-011545), 2001.

[3] G. Rizzoni y X.C. Chen. "Detection of internal combustion engine knock using time-frequency distributions". In Circuits and Systems, 1993., Proceedings of the 36th Midwest Symposium on, pages 360 -363 vol.1, aug 1993.

[4] D. Konig y J.F. Bohme. "Application of cyclostationary and time-frequency signal analysis to car engine diagnosis". In Acoustics, Speech, and Signal Processing, 1994. ICASSP-94., 1994 IEEE International Conference on, volume iv, pages IV/149 -IV/152 vol.4, apr 1994.

[5] B. Samimy y G. Rizzoni. "Mechanical signature analysis using time-frequency signal processing: application to internal combustion engine knock detection". Proceedings of the IEEE, 84(9):1330 -1343, sep 1996.

[6] S. Kim y K. Min. "Detection of combustion start in the controlled auto ignition engine by wavelet transform of the engine block vibration signal". Measurement Science and Technology, 19(8):085407, 2008.

[7] L. Cohen. Time-Frequency Analysis. Prentice Hall Signal Processing Series. Prentice Hall PTR, 1995.

[8] S. Qian y D. Chen. Joint time-frequency analysis: methods and applications. Joint Time-frequency Analysis: Methods and Applications. PTR Prentice Hall, 1996.

[9] B. Boashash. Time-Frequency Signal Analysis and Processing: A Comprehensive Reference. Elsevier, 2003. 
[10] N. Cavina y F. Ponti. "Air fuel ratio estimation using in-cylinder pressure frequency analysis". Journal of Engineering for Gas Turbines and Power, 125(3):812-819, 2003.

[11] V. Macián, J. Galindo, J.M. Luján, y C. Guardiola. "Detection and correction of injection failures in diesel engines on the basis of turbocharger instantaneous speed frequency analysis". Proceedings of the Institution of Mechanical Engineers, Part D: Journal of Automobile Engineering, 219(5):691-701, 2005.

[12] J. Arregle, J.J. Lopez, J.M. Garcia, y C. Fenollosa. "Development of a zerodimensional diesel combustion model. part 1: Analysis of the quasi-steady diffusion combustion phase". Applied Thermal Engineering, 23(11):1301 - 1317, 2003.

[13] J. Arregle, J.J. Lopez, J.M. Garcia, y C. Fenollosa. "Development of a zerodimensional diesel combustion model: Part 2: Analysis of the transient initial and final diffusion combustion phases". Applied Thermal Engineering, 23(11):1319 $1331,2003$.

[14] C. Fenollosa. Aportación a la descripción fenomenológica de proceso de combustión por difusión diesel. Tesis Doctoral, Universidad Politécnica de Valencia, 2003.

[15] J. Galindo, J.M. Lujan., J.R. Serrano, y L. Hernández. “Combustion simulation of turbochargers hsdi diesel engines during transient operation using neuronal networks". Applied Thermal Engineering, 25:877-898, 2005.

[16] S. X. Chen y J. J. Moskwa. "Application of nonlinear sliding-mode observers for cylinder pressure reconstruction”. Control Engineering Practice, 5(8):1115 - 1121, 1997.

[17] R. Bracewell. The Fourier Transform \& Its Applications. McGraw-Hill Science/Engineering/Math, 3rd edition, 1999.

[18] A. Antoniou. Digital Signal Processing: Signals, Systems, and Filters. McGraw-Hill Professional, Octubre 2005.

[19] E.L. Kosarev y E. Pantos. “Optimal smoothing of'noisy'data by fast fourier transform”. Journal of Physics E: Scientific Instruments, 16(6):537, 1983. 
Capítulo 7

\section{Conclusiones y trabajos futuros}




\section{Índice}

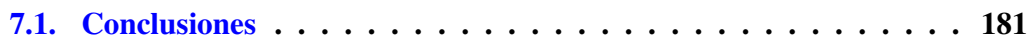

7.2. Trabajos futuros $\ldots \ldots \ldots \ldots \ldots \ldots$ 


\subsection{Conclusiones}

El trabajo presentado en esta tesis ha estado encaminado a la consecución de los objetivos generales enunciados en el capítulo 1, esto es, definir las metodologías experimentales para la puesta a punto de herramientas de medida y tratamiento digital de la señal de presión en cámara y parametrización rápida de fenómenos relacionados con la inyección-combustión que se producen dentro del cilindro, mediante tratamiento digital de la señal en dominio temporal y en dominio de frecuencias.

Para ello:

- Se han descrito las herramientas actuales de gestión de los motores diésel, la importancia que tiene la presión en cámara y el papel significativo que tiene en el desarrollo de futuras estrategias de control de dichos motores.

- Se ha justificado la elección de la herramienta necesaria para la medida de la presión en cámara teniendo en cuenta el uso final que se le va a dar a dicha medida.

- Se ha contribuido en el tratamiento digital de la señal medida, aportando una mejora en la precisión del diagnóstico de la combustión a partir de presiones en cámara filtradas con criterios experimentales robustos. De esta forma, se ha logrado un diagnóstico de la combustión que ha sido referencia para validación de las metodologías de diagnóstico que se plantearon más adelante.

- Se han presentado nuevas metodologías para la parametrización de la combustión basadas en el tratamiento digital de la señal de la derivada de la presión en cámara. Se han discutido los resultados experimentales obtenidos por cada metodología propuesta para determinar la capacidad, las ventajas y los inconvenientes de cada metodología

Así se ha llegado a las siguientes conclusiones:

1. Las ECUs actuales no son capaces de llevar a cabo un diagnóstico de la combustión preciso, no sólo por falta de sensores, sino también por la limitación de memoria y de poder computacional de las propias ECUs. Analizando las estrategias de control implementadas en dichas ECUs se aprecia como estas últimas gestionan los motores diésel actuales mediante estrategias que evalúan el estado de las variables físicas instante a instante y no a lo largo de ciclos del motor.

2. El aprovechamiento óptimo de la energía del combustible inyectado, y con la menor contaminación posible, pasa forzosamente por un control del proceso de la combustión ciclo a ciclo. Para ello se requiere una selección cuidadosa de los sistemas de medida de la señal de presión en cámara, no sólo para abaratar los costes, sino también para diagnosticar de una forma optima la combustión.

3. Las justificaciones expuestas en la presente tesis han determinado criterios para la selección de los sistemas de medida. Dichos criterios pueden resumirse en: 
- Para el campo de investigación los sensores más fiables para la medición de la presión en cámara son los piezoeléctricos de alta sensibilidad y baja deriva térmica.

- La resolución mínima para el registro de la señal es de 12 bits.

- La frecuencia de muestreo recomendada para estudios de la combustión es $12 \mathrm{kHz}$ para el caso de muestreo temporal o una equivalente en función del régimen de giro del motor para el caso de muestreo angular.

- Para el óptimo diagnóstico de la combustión, se exige el muestreo angular. En este caso, se ha contribuido con la metodología del tratamiento de la señal medida, argumentando la elección del número de ciclos a promediar para la etapa previa al filtrado y detallando los criterios y la metodología experimental de la selección de los parámetros del filtro digital. De esta forma, se logra una baja desviación típica en los resultados, principalmente en la máxima desviación típica de la dFQL en puntos de funcionamiento estables del motor (inferior a $0.1 \mathrm{~J} /{ }^{\circ}$ ).

- Para el caso de aplicación del filtrado en tiempo real, se han establecido los parámetros del filtro paso bajo, permitiendo obtener resultados similares a los obtenidos en el filtrado offline. Dichos parámetros son función del régimen de giro y el grado de carga del motor.

- El método de filtrado en tiempo real puede ser utilizado para mejorar las herramientas tradicionales del diagnóstico de la combustión. Dichas herramientas han sido desarrolladas fundamentalmente para el diagnóstico de la combustión en motores en condiciones de funcionamiento estacionarias. Los parámetros de los filtros propuestos aportan una alternativa para el diagnóstico en condiciones de funcionamiento transitorias.

4. Para estudios basados en la derivada de la presión en cámara $(d p)$, se ha propuesto una alternativa de medida de la derivada de la señal de la presión en cámara. Dicha alternativa, pese a su limitación, ha sido implementada, cómodamente, para el diagnóstico en tiempo real de parámetros de la combustión basados en el estudio temporal de la derivada de presión en cámara.

5. Los cambios bruscos en la forma de la señal de $d p$ están estrechamente relacionados con eventos asociados a la combustión habiéndose llegado a las siguientes conclusiones:

- Los mínimos locales de la dp, después del inicio de la inyección, están directamente relacionados con el inicio de la combustión. Dichos mínimos, son más fáciles de localizar para combustiones enérgicas, principalmente las combustiones principales.

- En los alrededores del ángulo donde se produce la máxima tasa de liberación de calor (dFQLmax), se alcanzan las máximas magnitudes y las máximas frecuencias presentes en la dp. 
- Al final de la combustión la liberación de calor deja de alterar el comportamiento de la $d p$ atenuando la magnitud de los componentes en frecuencia de la señal y por lo tanto su rizado.

6. Las metodologías, establecidas y ajustadas, basadas en el análisis temporal de la $d p$ para evaluar los cambios bruscos de la $d p$ requieren un coste computacional despreciable y aportan resultados satisfactorios con el mínimo número de parámetros de ajuste. Dichas metodologías han sido validadas en todo tipo de condiciones de funcionamiento de varios motores. Las validaciones han mostrado que:

- La localización del ángulo del SoC principal es altamente fiable mediante la detección directa del mínimo local de la dp

- La localización del ángulo del SoC piloto se logra combinando la detección del mínimo local correspondiente con la amplitud del rizado de la dp en dichos mínimos. Teniendo en cuenta el poco calor liberado en combustiones piloto, los resultados obtenidos con esta metodología son fiables si se considera aceptable un error máximo de $4^{\circ}$ en la localización del SoC piloto.

- La localización del EoC, a partir de la evaluación del rizado de la señal de dp, aporta, en casi el $80 \%$ de los casos, resultados relativos a un rango de liberación de calor entre el 80 y el $97.5 \%$ del total liberado durante el ciclo. Dado que sólo se maneja información contenida en la señal de la dp, el ángulo del EoC estimado con esta metodología no tiene en cuenta la transmisión de calor a las paredes del cilindro. Por lo que quedan justificadas las diferencias entre los resultados obtenidos con esta metodología y los resultados de referencia obtenidos mediante el modelo de diagnóstico CALMEC.

- La localización del ángulo de la dFQLmax es fiable teniendo en cuenta el ángulo en que se localiza la dpmax. El error cometido es inferior a $2^{\circ}$ en el $80 \%$ de los casos.

7. En cuando a la metodología de detección basada en el análisis conjunto tiempofrecuencia, los resultados son similares a los obtenidos mediante el análisis en dominio temporal. La mejora en la precisión del EoC no compensa el coste computacional considerable que supone la metodología, sin embargo, esta metodología brinda interfaces gráficos a tener en cuenta para el diagnóstico de la combustión online en salas de ensayo de motores.

8. Por otra parte, se ha manifestado el potencial del análisis en frecuencia para la obtención una buena aproximación de la ley de liberación de calor y otros parámetros (pmi, $F Q L$, etc.), partiendo únicamente de la señal de presión o su derivada y de la geometría del motor. Se han sugerido e implementado algunos ejemplos de aplicación acompañados con resultados que muestran que el análisis en frecuencia de la presión en cámara sigue siendo un aspecto a explorar. 


\subsection{Trabajos futuros}

A lo largo de esta tesis se han abordado aspectos dirigidos al diagnóstico de la combustión en tiempo real, donde se han tenido muy en cuenta las limitaciones computacionales de las ECUs actuales y los tiempos necesarios para llevar a cabo el diagnóstico de la combustión con los mininos recursos. Por estos motivos, algunos aspectos no han podido desarrollarse de forma completa o son demasiado ambiciosos para incluirse en un sólo trabajo. Merecen ser destacados los siguientes:

1. El estudio del proceso de la combustión desde el punto de vista temporal, es decir, adquirir la señal de presión en cámara con frecuencias de muestreo temporales (no angulares) adecuadas y efectuar el diagnóstico de la combustión. De esta forma, se podría relacionar con mayor precisión las frecuencias que componen la señal de presión en cámara, con fenómenos que se producen dentro del cilindro.

2. El estudio de las frecuencias que componen la señal de presión en cámara puede aportar, además de información de eventos relacionados con la combustión, información del contenido del cilindro en cada ciclo de motor. Por lo tanto, se propone este tipo de estudio en trabajos futuros.

3. El diseño de nuevas estrategias de control, implementando las metodologías presentadas en esta tesis, permitirá cotejar las teorías de control de motores diésel, expuestas en los foros científicos, con estudios experimentales mediante la optimización del proceso de la combustión. Se propone como trabajo a realizar, la aplicación de las metodologías estudiadas en esta tesis mediante la integración de las mismas en las unidades de control electrónico de los motores actuales. 


\section{Bibliografía general}

\section{A. Van Aken.}

Adapter errors in indicator diagrams of combustion engines.

IMechE, 191,8/77, pp. 125-134, 1977.

(citado 2 veces en pp 55 y 61.)

\section{A. Antoniou.}

Digital Signal Processing: Signals, Systems, and Filters.

McGraw-Hill Professional, Octubre 2005.

(citado 5 veces en pp 63, 65, 113, 118 y 166.)

O. Armas.

Diagnostico experimental del proceso de combustión en motores Diesel de inyección directa.

Tesis Doctoral, Universidad Politécnica de Valencia, Valencia, Spain, 1998.

(citado 10 veces en pp 10, 44, 48, 62, 66, 84, 86, 97, 105 y 115.)

\section{J. Arregle, J.J. Lopez, J.M. Garcia, y C. Fenollosa.}

Development of a zero-dimensional Diesel combustion model. Part 1: Analysis of the quasi-steady diffusion combustion phase.

Applied Thermal Engineering, 23(11), pp. 1301 - 1317, 2003.

(citado en $\mathrm{p}$ 166.)

\section{J. Arregle, J.J. Lopez, J.M. Garcia, y C. Fenollosa.}

Development of a zero-dimensional Diesel combustion model: Part 2: Analysis of the transient initial and final diffusion combustion phases.

Applied Thermal Engineering, 23(11), pp. 1319 - 1331, 2003.

(citado en $\mathrm{p}$ 166.)

R. A. Atkins, J. H. Gardner, W. N. Gibler, C. E. Lee, M. D. Oakland, M. O. Spears, V. P. Swenson, H. F. Taylor, J. J. McCoy, y G. Beshouri.

Fiber-optic pressure sensors for internal combustion engines.

Applied Optics, 33, pp. 1315-1320, 1994.

(citado en p 53.) 
A. Baccile, D. Ceccarini, L. Cheng, A. Iacoponi, T. Lake, A. Noble, y J. Stokes. An Innovative Control System for a 2/4 Stroke Switchable Engine.

SAE paper, (2007-01-1199), 2007.

(citado en $\mathrm{p} 33$. )

M. Beasley, R. Cornwell, P. Fussey, R. King, A. Noble, T. Salamon, y A. Truscott. Reducing diesel emissions dispersion by coordinated combustion feedback control.

SAE paper, (2006-01-0186), 2006.

(citado 2 veces en pp 45 y 46. )

\section{P.R. Bevington y D. Robinson.}

Data Reduction and Error Analysis for the Physical Sciences.

McGraw-Hill, 3rd edition, 2002.

(citado 2 veces en pp 64 y 98.)

B. Boashash.

Time-Frequency Signal Analysis and Processing: A Comprehensive Reference.

Elsevier, 2003.

(citado 3 veces en pp 113, 161 y 166.)

\section{R. Bracewell.}

The Fourier Transform \& Its Applications.

McGraw-Hill Science/Engineering/Math, 3rd edition, 1999.

(citado 3 veces en pp 99, 102 y 166.)

M.F.J. Brunt y A.L. Emtage.

Evaluation of IMEP routines and analysis errors.

SAE paper, (960609), 1996.

(citado en $\mathrm{p}$ 105.)

\section{M.F.J. Brunt y C.P. Kieron.}

Calculation of heat release in Direct Injection diesel engines.

SAE paper, (1999-01-0187), 1999.

(citado en $\mathrm{p}$ 44.)

\section{M.F.J. Brunt y G.G. Lucas.}

The Effect of Crank Angle Resolution on Cylinder Pressure Analysis.

SAE paper, (910041), 1991.

(citado en $\mathrm{p}$ 46.)

\section{M.F.J. Brunt, H. Rai, y A.L. Emtage.}

The calculation of heat release energy from engine cylinder pressure data.

SAE paper, (981052), 1998.

(citado en $\mathrm{p} 44$. 
A. Cartwight y R. Fleck.

Cylinder pressure analysis in high performance two stroke engines.

SAE paper, (962535), 1996.

(citado en p 105.)

N. Cavina y F. Ponti.

Air Fuel Ratio Estimation Using In-Cylinder Pressure Frequency Analysis.

Journal of Engineering for Gas Turbines and Power, 125(3), pp. 812-819, 2003.

(citado en $\mathrm{p}$ 163.)

\section{S. X. Chen y J. J. Moskwa.}

Application of nonlinear sliding-mode observers for cylinder pressure reconstruction.

Control Engineering Practice, 5(8), pp. 1115 - 1121, 1997.

(citado en p 166.)

L. Cohen.

Time-Frequency Analysis.

Prentice Hall Signal Processing Series. Prentice Hall PTR, 1995.

(citado 3 veces en pp 113, 161 y 163.)

\section{J. A. Cook y B. K. Powell.}

Modeling of an internal combustion engine for control analysis.

Control Systems Magazine, IEEE, 8(4), pp. 20 -26, aug 1988.

(citado en $\mathrm{p}$ 30.)

\section{J.W. Cooley, P.A.W. Lewis, y P.D. Welch.}

The Fast Fourier Transform and Its Applications.

Education, IEEE Transactions on, 12(1), pp. 27 -34, march 1969.

(citado 2 veces en pp 102 y 104.)

\section{E. Corti, D. Moro, y L. Solieri.}

Real-Time Evaluation of IMEP and ROHR-related Parameters.

SAE paper, 2007-24-0068(2007-24-0068), 2007.

(citado en $\mathrm{p}$ 46.)

\section{R.S. Davis y G.J. Paterson.}

Cylinder pressure data quality checks and procedures to maximize data accuracy.

SAE paper, (2006-01-1346), 2006.

(citado en $\mathrm{p} 46$.

\section{J.M. Desantes, J. Galindo, C. Guardiola, y V. Dolz.}

Air mass flow estimation in turbocharged diesel engines from in-cylinder pressure measurement.

Experimental Thermal and Fluid Science, 34, pp. 37-47, 2009.

(citado en $\mathrm{p}$ 42.) 
J.M. Desantes, A.J. Torregrosa, y A. Broatch.

Wavelet Transform applied to Combustion Noise Analysis in High-speed DI Diesel Engines.

SAE paper, (2001-01-1545), 2001.

(citado 3 veces en pp 117, 130 y 153.)

G. Dober, S. Tullis, G. Greeves, N. Milovanovic, M. Hardy, y S. Zuelch.

The Impact of Injection Strategies on Emissions Reduction and Power Output of Future Diesel Engines.

SAE paper, (2008-01-0941), 2008.

(citado en $\mathrm{p}$ 31.)

\section{R. Douglas, R.J. Kee, y B.P. Carberry.}

Analysis of chamber pressure data in two stroke engines.

SAE paper, (972792), 1997.

(citado 2 veces en pp 46 y 90.)

\section{Fenollosa.}

Aportación a la descripción fenomenológica de proceso de combustión por difusión diesel.

Tesis Doctoral, Universidad Politécnica de Valencia, 2003.

(citado 3 veces en pp 41, 77 y 166.)

\section{A. Ferrari, M. Chiodi, M. Bargende, y P. et al. Roberti.}

Virtual Set-up of a Racing Engine for the Optimization of Lap Performance through a Comprehensive Engine-Vehicle-Driver Model.

SAE paper, (2011-24-0141), 2011.

(citado en $\mathrm{p}$ 33.)

\section{Fuji, S. Yagi, M. Kawai, y H. Yoshikawa.}

MBT Control Utilizing Crank Angle of Maximum Combustion Pressure.

SAE paper, (890759), 1989.

(citado en $\mathrm{p} 45$.

J. Galindo, J.M. Lujan., J.R. Serrano, y L. Hernández.

Combustion simulation of turbochargers HSDI Diesel engines during transient operation using neuronal networks.

Applied Thermal Engineering, 25, pp. 877-898, 2005.

(citado 2 veces en pp 33 y 166.)

\section{J.M. García.}

Aportación al estudio del proceso de combustión turbulenta de chorros en motores diesel de inyección directa.

Tesis Doctoral, Universidad Politécnica de Valencia, 2004.

(citado en p 91.) 


\section{A. Garcia Martinez.}

Estudio de los efectos de la post inyección sobre el proceso de combustión y la formación de hollín en motores diésel.

Tesis Doctoral, Universidad Politécnica de Valencia, 2009.

(citado en p 31.)

J.A. Gatowski, E.N. Balles, K.M. Chun, J.A.F.E. Nelson, E. hian, y J.B Heywood. Heat release analysis of engine pressure data.

SAE paper, (841359), 1984.

(citado en p 42.)

\section{G. Gautschi.}

Piezoelectric sensorics: force, strain, pressure, acceleration and acoustic emission sensors, materials and amplifiers.

Engineering online library. Springer, 2002.

(citado 2 veces en pp 54 y 59.)

\section{R.C. González y R.E. Woods.}

Tratamiento digital de imágenes.

Addison Wesley Iberoamericana, 1996.

(citado en $\mathrm{p}$ 104.)

\section{Carlos Guardiola.}

Detección y compensación de irregularidades de inyección a través de la medida del régimen instantáneo del turbogrupo.

Temas avanzados en motores de combustión interna. Editorial Reverté, S.A., 2005. (citado en p 79.)

\section{F.J. Harris.}

On the use of windows for harmonic analysis with the discrete Fourier transform.

Proceedings of the IEEE, 66, pp. 51-83, 1978.

(citado en $\mathrm{p}$ 114.)

M. Hasegawa, Y. Shimasaki, S. Yamaguchi, M. Kobayashi, H. Sakamoto, N. Kitayama, y T. Kanda.

Study on ignition timing control for Diesel engines using in-cylinder pressure sensor.

SAE paper, (2006-01-0180), 2006.

(citado 2 veces en pp 33 y 46.)

\section{Y. Hata, K. Ikeura, T. Morita, y T. Abo.}

Engine control system using a cylinder pressure sensor.

IEE PROCEEDINGS, 136, 1989.

(citado en $\mathrm{p}$ 45.)

\section{E. Hendricks y J. Luther.}

Model and Observer Based Control of Internal Combustion Engines.

In Modeling, Emissions and Control in Automotive Engines, 2001.

(citado en p 30.) 


\section{Elbert Hendricks y Spencer C. Sorenson.}

Mean Value Modelling of Spark Ignition Engines.

SAE paper, (900616), 1990.

(citado en $\mathrm{p} 30$. )

\section{J.B. Heywood.}

Internal combustion engine fundamentals.

McGraw-Hill,Inc. ISBN 0-07-100499-8, 1988.

(citado 4 veces en pp 41, 44, 88 y 90.)

\section{G. Hohenberg.}

Experimentelle Erfassung der Wandwarme in Kolbenmotoren (Experimental acquisition of the wall heat in piston engines).

Tesis Doctoral, Technical University of Graz, Graz, Austria, 1980.

(citado en p 84.)

\section{S.C. Homsy y A. Atreya.}

An experimental heat release rate analysis of a Diesel engine operating under steady conditions.

SAE paper, (970889), 1997.

(citado en p 56.)

\section{D.T. Hountalas y A. Anestis.}

Effect of pressure transducer position on measured cylinder pressure diagram of high speed diesel engines.

Energy Conversion and Management, 39, pp. 589-607., 1998.

(citado en p 55.)

\section{H.L. Husted, D. Kruger, G. Fattic, G. Ripley, y E. Kelly.}

Cylinder pressure-based control of pre-mixed diesel combustion.

SAE paper, (2007-01-0773), 2007.

(citado en $\mathrm{p}$ 46.)

\section{H. Jin, S. Choi, H. Jin-Kaist, y S. Choi-Kaist.}

Sliding Sector Mode Control with Linear Parameter Varying Diesel Engine Model.

SAE paper, (2011-01-0696), 2011.

(citado en $\mathrm{p} 33$.

\section{T.V. Johnson.}

Diesel emission control in review.

SAE paper, 1(2008-01-0069), pp. 68-81, 2008.

(citado en $\mathrm{p} 33$. )

\section{R. Johnsson.}

Cylinder pressure reconstruction based on complex radial basis function networks from vibration and speed signals.

Mechanical Systems and Signal Processing, 20, pp. 1923-1940, 2006.

(citado en p 33.) 


\section{S. Kim y K. Min.}

Detection of combustion start in the controlled auto ignition engine by wavelet transform of the engine block vibration signal.

Measurement Science and Technology, 19(8), pp. 085407, 2008.

(citado 2 veces en pp 161 y 162.)

\section{Komachiya, H. Sonobe, S. Oho, M. Kurita, T. Nakazawa, y T. Sasayama.}

Multiplex in-cylinder pressure measurement utilizing an optical fiber with specific refractive-index composition.

Applied Optics, 35 - 7, pp. 1143-1150, 1996.

(citado en p 53.)

\section{Konig y J.F. Bohme.}

Application of cyclostationary and time-frequency signal analysis to car engine diagnosis.

In Acoustics, Speech, and Signal Processing, 1994. ICASSP-94., 1994 IEEE International Conference on, volume iv, pp. IV/149 -IV/152 vol.4, apr 1994.

(citado en p 161.)

\section{E.L. Kosarev y E. Pantos.}

Optimal smoothing of'noisy'data by fast Fourier transform.

Journal of Physics E: Scientific Instruments, 16(6), pp. 537, 1983.

(citado 2 veces en pp 116 y 166.)

\section{R.H. Kuratle y B. Märki.}

Influencing parameters and error sources during indication on internal combustion engines.

SAE paper, (920233), 1992.

(citado en $\mathrm{p}$ 46.)

\section{D.R. Lancaster, R.B. Krieger, y J.H. Lienesch.}

Measurements and analysis of engine pressure data.

SAE paper, (750026), 1975.

(citado 4 veces en pp 44, 46, 56 y 105.)

\section{Lapuerta.}

Un modelo de combustión fenomenológico para un motor Diesel de inyección directa rápido.

Tesis Doctoral, Universidad Politécnica de Valencia, 1988.

(citado en $\mathrm{p}$ 77.)

\section{Lapuerta, O. Armas, y V. Bermúdez.}

Sensitivity of Diesel engine thermodynamic cycle calculation to measurement errors and estimated parameters.

Applied Thermal Engineering, 20, pp. 843861, 2000.

(citado 2 veces en pp 48 y 97.) 


\section{Lapuerta, O. Armas, y J.J. Hernández.}

Diagnosis of DI Diesel combustion from in-cylinder pressure signal by estimation of mean thermodynamic properties of the gas.

Applied Thermal Engineering, 19, pp. 513-529, 1999.

(citado 4 veces en pp 41, 47, 86 y 97.)

\section{Lapuerta, O. Armas, y S. Molina.}

Study of the compression cycle of a reciprocating engine through the polytropic coefficient.

Applied Thermal Engineering, 23, pp. 313-323, 2003.

(citado en $\mathrm{p}$ 114.)

S. Lee, C. Bae, R. Prucka, G. Fernandes, Z.S. Filipi, y D.N. Assanis.

Quantification of thermal shock in a piezoelectric pressure transducer.

SAE paper, 114, No. 3(2005-01-2092), pp. 1370-1381, 2005.

(citado en $\mathrm{p}$ 106.)

\section{S. Leonhardt, N. Müller, y R. Isermann.}

Methods for engine supervision and control based on cylinder pressure information.

IEEE/ASME Transactions on Mechatronic, 4, pp. 235-245, 1999.

(citado 4 veces en pp 45, 55, 90 y 130.)

\section{J.M. Luján, H. Climent, C. Guardiola, y J.V. García-Ortiz.}

A comparison of different algorithms for boost pressure control in a heavy duty turbocharged diesel engine.

Proc. Inst. Mech. Eng. Part D, Journal of Automobile Eng., 220, pp. 629-640, 2007.

(citado en $\mathrm{p}$ 33.)

\section{Macian.}

Contribución al estudio de la influencia del movimiento del ruido sobre la dispersión cíclica y su caracterización.

Tesis Doctoral, Universidad Politécnica de Valencia, 1984.

(citado 5 veces en pp 10, 48, 77, 105 y 106.)

\section{Macián, J. Galindo, J.M. Luján, y C. Guardiola.}

Detection and Correction of injection failures in diesel engines on the basis of turbocharger instantaneous speed frequency analysis.

Proceedings of the Institution of Mechanical Engineers, Part D: Journal of Automobile Engineering, 219(5), pp. 691-701, 2005.

(citado en $\mathrm{p}$ 163.)

\section{J. Martín.}

Aportación al diagnóstico de la combustión en motores Diesel de inyección directa. Tesis Doctoral, Universidad Politécnica de Valencia, 2007.

(citado 15 veces en pp 10, 41, 44, 45, 46, 48, 62, 66, 77, 86, 91, 97, 102, 106 y 115.) 
J. Martín.

Diagnóstico de la combustión en motores diésel de inyección directa.

ISBN: 978-84-291-4717-9, 2012.

(citado 3 veces en pp 41, 44 y 48.)

T. Minami, K. Takeuchi, y N. Shimazaki.

Reduction of Diesel Engine NOx Using Pilot Injection.

SAE paper, (950611), 1995.

(citado en p 31.)

R. Müller, M. Hart, A. Truscott, A. Noble, G. Krötz, M. Eickhoff, C. Cavalloni, y M. Gnielka.

Combustion pressure based engine management system.

SAE paper, (2000-01-0928), 2000.

(citado en $\mathrm{p} 45$.

\section{Morishita y T. Kushiyama.}

An improved method for determining the TDC position in a $\mathrm{pV}$-diagram.

SAE paper, (980625), 1998.

(citado 2 veces en pp 44 y 46.)

\section{S. Nakayama, T. Ibuki, H. Hosaki, y H. Tominaga.}

An Application of Model Based Combustion Control to Transient Cycle-by-Cycle Diesel Combustion.

SAE paper, (2008-01-1311), 2009.

(citado en p 33.)

\section{A. Nehmer y R. D. Reitz.}

Measurement of the Effect of Injection Rate and Split Injections on Diesel Engine Soot and NOx Emissions.

SAE paper, (940668), 1994.

(citado en p 31.)

\section{G.H. Neo y N. Collings.}

Pressure data analysis of formula one racing engines.

SAE paper, (970061), 1997.

(citado en p 56.)

\section{Nieuwstadt y J. Michiel.}

Method and system for controlling EGR rate in diesel engines.

Patente, Estados Unidos, May 2004.

(citado en $\mathrm{p}$ 32.)

\section{E. Oliver.}

Estudio de sistemas de adquisición y tratamiento de datos en motores de combustión interna alternativos.

Tesis Doctoral, Universidad Politécnica de Valencia, Valencia, 1991.

(citado 6 veces en pp 10, 48, 56, 62, 84 y 105.) 


\section{F. Payri, J. Arrègle, J.J. López, y E. Mocholí.}

Diesel NOx Modeling with a Reduction Mechanism for the Initial NOx Coming from EGR or Re-entrained Burned Gases.

SAE paper, (2008-01-1188), 2008.

(citado en p 33.)

\section{F. Payri, A. Broatch, B. Tormos, y V. Marant.}

New methodology for in-cylinder pressure analysis in direct injection diesel engines application to combustion noise.

Measurement Science and Technology, 16, pp. 540-547, 2005.

(citado 4 veces en pp 62, 65, 66 y 117.)

\section{F. Payri y J.M. DESANTES.}

Motores de combustión interna alternativos.

isbn: 978-84-291-4802-2, 2011.

(citado 3 veces en pp 41, 44 y 88.)

\section{F. Payri, J.M. Lujan, J. Martin, y A. Abbad.}

Digital signal processing of in-cylinder pressure for combustion diagnosis of internal combustion engines.

Mechanical Systems and Signal Processing, 24(6), pp. 1767 - 1784, 2010.

(citado en $\mathrm{p}$ 117.)

\section{F. Payri, S. Molina, J. Martín, y O. Armas.}

Influence of measurement errors and estimated parameters on combustion diagnosis. Applied Thermal Engineering, 26, pp. 226-236, 2006.

(citado 4 veces en pp 11, 48, 77 y 82.)

\section{F. Payri, P. Olmeda, C. Guardiola, y J. Martín.}

Adaptive determination of cut-off frequencies for filtering the in-cylinder pressure in diesel engines combustion analysis.

Applied Thermal Engineering, In Press, Corrected Proof, pp. -, 2011.

(citado en p 117.)

\section{A. Pierpont y R. D. Reitz.}

Effects of Injection Pressure and Nozzle Geometry on D.I. Diesel Emissions and Performance.

SAE paper, (950604), 1995.

(citado en p 31.)

\section{S. Qian y D. Chen.}

Joint time-frequency analysis: methods and applications.

Joint Time-frequency Analysis: Methods and Applications. PTR Prentice Hall, 1996.

(citado en $\mathrm{p}$ 161.) 
C. Quérel, O. Grondin, y C. Letellier.

State of the Art and Analysis of Control Oriented NOx Models.

SAE paper, (2012-01-0723), 2012.

(citado en $\mathrm{p} 33$.

\section{H.S. Rai, M.F.J. Brunt, y C.P. Loader.}

Quantification and reduction of IMEP errors resulting from pressure transducer thermal shock in a SI engine.

SAE paper, (1999-01-1329), 1999.

(citado en $\mathrm{p}$ 46.)

\section{A.L. Randolph.}

Methods of processing cylinder-pressure transducer signals to maximize data accuracy.

SAE paper, (900170), 1990.

(citado en $\mathrm{p}$ 105.)

\section{R. Reitz y J. von der Ehe.}

Use of in-cylinder pressure measurement and the response surface method for combustion feedback control in a diesel engine.

IMechE, 220 Part D, 2006.

(citado en p 90.)

\section{E. Ya. Remez.}

Sur la détermination des polynômes d'approximation de degré donnée.

Comm. Soc. Math. Kharkov, 10, pp. 41, 1934.

(citado en p 118.)

\section{G. Rizzoni y X.C. Chen.}

Detection of internal combustion engine knock using time-frequency distributions.

In Circuits and Systems, 1993., Proceedings of the 36th Midwest Symposium on, pp. 360 -363 vol.1, aug 1993.

(citado 2 veces en pp 161 y 162.)

\section{E. Rosseel, R. Sierens, y R.S.G. Baert.}

Evaluating piezo-electric transducer response to thermal shock from in-cylinder pressure data.

In SAE Conference, Detroit, 1999, 1999.

(citado 2 veces en pp 51 y 61.)

\section{B. Samimy y G. Rizzoni.}

Mechanical signature analysis using time-frequency signal processing: application to internal combustion engine knock detection.

Proceedings of the IEEE, 84(9), pp. 1330-1343, sep 1996.

(citado en $\mathrm{p}$ 161.) 
S. Saraswati y S. Chand.

Reconstruction of cylinder pressure for SI engine using recurrent neural network.

Neural Computing \&amp; Applications, 19, pp. 935-944, 2010.

10.1007/s00521-010-0420-6.

(citado en p 33.)

D. Schiefer, R. Maennel, y W. Nardoni.

Advantages of diesel engine control using in-cylinder pressure information for closed loop control.

SAE paper, (2003-01-0364), pp. 01-0364, 2003.

(citado en $\mathrm{p} 34$. )

M. C. Sellnau, F. A. Matekunas, P. A. Battiston, C.Chang, y D.R. Lancaster.

Cylinder-pressure-based engine control using pressure-ratio-management and lowcost non-intrusive cylinder pressure sensors.

SAE paper, (2000-01-0932), 2000.

(citado en $\mathrm{p}$ 90.)

\section{S.X. Shi y H.Z. Sheng}

Numerical simulation and digital signal processing in measurements of cylinder pressure of internal combustion engines.

IMechE, C20/87, pp. 211-218, 1987.

(citado en $\mathrm{p}$ 113.)

Y. Shimasaki, M. Kobayashi, H. Sakamoto, M. Ueno, M. Hasegawa, S. Yamaguchi, y T. Suzuki.

Study on engine management system using chamber pressure sensor integrated with spark plug.

SAE paper, (2004-01-0519), 2004.

(citado 3 veces en pp 45, 46 y 54.)

\section{M.J. Stas.}

An universally applicable thermodynamic method for TDC determination.

SAE paper, (2000-01-0561), 2000.

(citado en $\mathrm{p}$ 66.)

\section{J. Tichý, J. Erhart, E. Kittinger, y J. Prívratská.}

Fundamentals of piezoelectric sensorics: mechanical, dielectric, and thermodynamical properties of piezoelectric materials.

Springer, Berlin ;:London, 2010.

(citado en p 54.)

\section{J. Tichy y G.Gautschi.}

Piezo-Elektrische Meßtechnik.

Springer, 1980.

(citado en p 62.) 


\section{F.V. Tinaut.}

Contribución al estudio del proceso de combustión en motores de encendido por compresión de inyección directa.

Tesis Doctoral, Universidad Politécnica de Valencia, 1986.

(citado 2 veces en pp 10 y 48.)

\section{B. Tormos.}

Diagnóstico de Motores diésel mediante el análisis del aceite usado.

REVERTÉ, S. A., 2005.

(citado en p 49.)

\section{A. J. Torregrosa, A. Broatch, X. Margot, V. Marant, y Y. Beauge.}

Combustion chamber resonances in direct injection automotive diesel engines: a numerical approach.

International Journal of Engine Research, 5(1), pp. 83-91, 2004.

(citado 2 veces en pp 44 y 65.)

\section{A. J. Torregrosa, A. Broatch., J. Martín, y L Monelletta.}

Combustion noise level assessment in direct injection Diesel engines by means of incylinder pressure components.

Measurement Science and Technology, 18(7), pp. 2131-2142, 2007.

(citado 3 veces en pp 44, 47 y 65.)

T. C. Tow, D. A. Pierpont, y R. D. Reitz.

Reducing Particulate and NOx Emissions by Using Multiple Injections in a Heavy Duty D.I. Diesel Engine.

SAE paper, (940897), 1994.

(citado en $\mathrm{p}$ 31.)

\section{T. Tsuchiya, S. Morishita, T. Enomoto, H. Sasaki, y M. Yoshimura.}

Revolution Control of Generator Diesel Engine by Neural Network Controller.

SAE paper, (2003-01-0365), 2003.

(citado en $\mathrm{p} 33$.

\section{P. Tunestål y M. Lewander.}

Model Predictive Control of Partially Premixed Combustion.

In Luigi Re, Frank Allgöwer, Luigi Glielmo, Carlos Guardiola, y Ilya Kolmanovsky, editors, Automotive Model Predictive Control, volume 402 of Lecture Notes in Control and Information Sciences, pp. 171-181. Springer London, 2010.

(citado en $\mathrm{p} 33$. )

\section{O. Ulrico, R. Wlodarczyk, y M.T. Wlodarczyk.}

High-accuracy low-cost cylinder pressure sensor for advanced engine controls.

SAE paper, (2001-01-0991), 2001.

(citado en $\mathrm{p}$ 42.) 


\section{A. Arnau Vives.}

Piezoelectric Transducers and Applications.

Springer, 2nd ed. edition, Noviembre 2010.

(citado en p 56.)

\section{Wendeker y T. Kaminski.}

Development of a fiber-optic sensor for the measurement of dynamic cylinder pressure in spark ignition engine.

Sensors, IEEE, (2005), pp. 8, 2005.

(citado en $\mathrm{p}$ 53.)

\section{M.T. Wlodarcyk.}

High accuracy glow plug-integrated cylinder pressure sensor for closed loop engine control.

SAE paper, (2006-01-0184), 2006.

(citado 2 veces en pp 52 y 56.)

\section{M.T. Wlodarczyk, T. Poorman, L. Xia, J. Arnold, y T. Coleman.}

InCylinder FiberOptic Pressure Sensors for Monitoring and Control of Diesel Engines.

SAE paper, (981913), pp. 36-42, 1998.

(citado en $\mathrm{p}$ 53.)

\section{J. Worm.}

An evaluation of several methods for calculating transient trapped mass with emphasis on the "Delta P" approach.

SAE paper, (2005-01-0990), 2005.

(citado en $\mathrm{p} 46$.

H. Xie, R. Stobart, P. Tunestal, L. Eriksson, Y. Huang, y P. Leteinturier.

Future Engine Control Enabling Environment Friendly Vehicle.

SAE paper, (2011-01-0697), 2011.

(citado 2 veces en pp 33 y 34.)

M. Yao, Q. Zhang, H. Liu, P. Zhang, Z. Lin, T. Lin, y J. Shen.

Diesel Engine Combustion Control: Medium or Heavy EGR?

SAE paper, (2010-01-1125), 2010.

(citado en $\mathrm{p} 33$. )

M. Yoon, K. Lee, M. Sunwoo, y B. Oh.

Cylinder Pressure Based Combustion Phasing Control of a CRDI Diesel Engine.

SAE paper, (2007-01-0772), april 2007.

(citado en $\mathrm{p}$ 90.)

\section{P. Yoon, S. Parkand M. Sunwoo, I. Ohm, y K.J. Yoon.}

Closed-loop control of spark advance and air-fuel ratio in SI engines using cylinder pressure.

SAE paper, (2000-01-0933), 2000.

(citado en $\mathrm{p} 45$. 